\title{
Modified swim test as a mouse depression paradigm of enhanced cognitive processing
}

Citation for published version (APA):

Markova, N. (2017). Modified swim test as a mouse depression paradigm of enhanced cognitive processing: the role of GSK3 $\beta$. [Doctoral Thesis, Maastricht University]. Maastricht University. https://doi.org/10.26481/dis.20170502nm

Document status and date:

Published: 01/01/2017

DOI:

10.26481/dis.20170502nm

Document Version:

Publisher's PDF, also known as Version of record

Document license:

Unspecified

\section{Please check the document version of this publication:}

- A submitted manuscript is the version of the article upon submission and before peer-review. There can be important differences between the submitted version and the official published version of record.

People interested in the research are advised to contact the author for the final version of the publication, or visit the DOI to the publisher's website.

- The final author version and the galley proof are versions of the publication after peer review.

- The final published version features the final layout of the paper including the volume, issue and page numbers.

Link to publication

\footnotetext{
General rights rights.

- You may freely distribute the URL identifying the publication in the public portal. please follow below link for the End User Agreement:

www.umlib.nl/taverne-license

Take down policy

If you believe that this document breaches copyright please contact us at:

repository@maastrichtuniversity.nl

providing details and we will investigate your claim.
}

Copyright and moral rights for the publications made accessible in the public portal are retained by the authors and/or other copyright owners and it is a condition of accessing publications that users recognise and abide by the legal requirements associated with these

- Users may download and print one copy of any publication from the public portal for the purpose of private study or research.

- You may not further distribute the material or use it for any profit-making activity or commercial gain

If the publication is distributed under the terms of Article $25 \mathrm{fa}$ of the Dutch Copyright Act, indicated by the "Taverne" license above, 


\title{
MODIFIED SWIM TEST AS A MOUSE DEPRESSION PARADIGM OF ENHANCED COGNITIVE PROCESSING: THE ROLE OF GSK3 $\beta$
}

\author{
DISSERTATION
}

to obtain the degree of Doctor at Maastricht University,

on the authority of the Rector Magnificus, Prof. dr. Rianne M. Letschert in accordance with the decision of the Board of Deans, to be defended in public on Tuesday $2^{\text {nd }}$ of May 2017 , at $14: 30$ hours

by

Nataliia Markova 


\section{Supervisors}

Prof.dr. H.W.M. Steinbusch

Prof.dr. K-P. Lesch, University of Wuerzburg, Germany

Co-supervisor

Dr. T.V. Strekalova

\section{Members of the Degree Committee}

Prof.dr. B.P.F. Rutten (Chairman);

Prof.dr. C. Grandfils, University of Liège, Belgium

Prof.dr. E.A. Joosten

Prof.dr. E.D. Ponomarev, Chinese University of Hong Kong, China Prof.dr. K.R.J. Schruers 


\section{CONTENTS:}

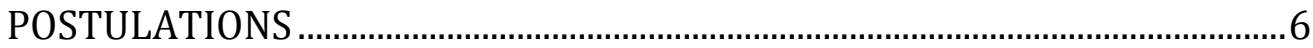

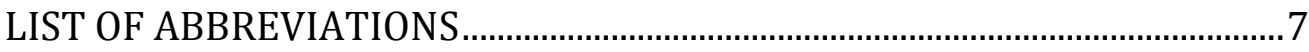

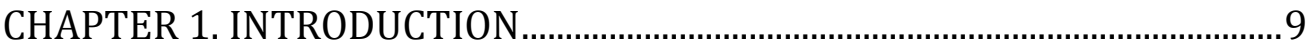

Enhanced cognitive processing as a pathophysiological mechanism of major depressive disorder .............................. 9

Animal models of enhanced memories for adversities .......... 12

Neuroanatomical circuits of pathologically enhanced learning of adversities during depression

Molecular and hormonal mechanisms of increased memory for adversities associated with depressive syndrome

GSK3 $\beta$ and molecular mechanisms of depression and neuronal plasticity

Forced swim and tail suspension tests as paradigms of depressive behaviour: implications for modeling of pathologically enhanced cognitive processing

Hypothesis and aim 25

References 26

CHAPTER 2. HIPPOCAMPAL GENE EXPRESSION OF DEIODINASES 2 AND 3 AND EFFECTS OF 3,5-DIIODO-LTHYRONINE T2 IN MOUSE DEPRESSION PARADIGMS

CHAPTER 3. A STUDY OF THE EFFECTS OF 3,5-DIIODO-LTHYRONINE IN THE TAIL SUSPENSION AND FORCED SWIM MODELS OF DEPRESSION 
CHAPTER 4. INDIVIDUAL DIFFERENCES IN BEHAVIOURAL DESPAIR PREDICT BRAIN GSK3 $\beta$ EXPRESSION IN MICE: THE POWER OF A MODIFIED SWIM TEST

CHAPTER 5. THIAMINE AND ITS PRECURSOR BENFOTIAMINE AMELIORATE COGNITIVE ABILITIES AND GSK3 $\beta$ RELATED DEPRESSIVE- AND ANXIETY-LIKE BEHAVIOURS IN MICE

CHAPTER 6. DISCUSSION AND SOCIETAL IMPACT. 176

Key factors in the modified swim test: the role of timing, adversity, repeated testing and contextual reminders

Changes of GSK3 $\beta$ brain activity as potential mechanism of enhanced learning, associated with depressive syndrome

Different roles of the hippocampus and the prefrontal cortex in behavioural and molecular changes in the modified swim test

Modeling of individual variability in the susceptibility to a depressive-like state with the modified swim test: a comparison with other paradigms.

A comparison of the modified swim test against other depression paradigms: pharmacological sensitivity, etiological relevance, face and construct validity 185

Advantages of modified swim test over other models of depression

Highlighting supplementary therapies of depression: the role of GSK3 $\beta$ in mechanisms of depression, enhanced learning of advertises and antidepressant therapies 
An outlook for further experiments to address the role of contextual conditioning in depression: studies with repeated testing in the tail suspension test

References

SUMMARY

VALORIZATION

ACKNOWLEDGMENTS

ABOUT THE AUTHOR

216

LIST OF PUBLICATIONS 


\section{Postulations}

1. Enhanced learning of adverse context is an important pathophysiological factor of a depressive syndrome (this thesis);

2. Its mechanisms can be mediated by over-expression of GSK3 $\beta$ in the prefrontal cortex in susceptible individuals (this thesis);

3. The hippocampus and prefrontal cortex play differential roles in GSK3 $\beta$-mediated contextual conditioning associated with depressive state (this thesis);

4. The modified swim test based on delayed testing can serve as a paradigm for abnormal acquisition of adverse memories during depression (this thesis);

5. Treatment with imipramine or thiamine (vitamin B1) prevents enhanced contextual conditioning of adversities and over-expression of brain GSK3 $\beta$ (this thesis);

6. Thyroid hormone $\mathrm{T} 2$ and thiamine (vitamin B1) can be exploited as new supplementary antidepressant therapies (this thesis);

7. "You must acquire the best knowledge first, and without delay; it is the height of madness to learn what you will later have to unlearn" (Erasmus; 1497);

8. "Learn the $A B C$ of science before you try to ascend to its summit" (Ivan Pavlov; 1936);

9. "Who reminds of past insults, is to lose an eye" / "Let sleeping dogs lie" (Russian proverb);

10. "To study, to study and once again, to study!" (Vladimir Lenin; 1924). 


\section{List of abbreviations}

Akt - protein kinase B

BDNF - brain-derived neurotrophic factor

CNS - central nervous system

$\mathrm{CR}$ - conditioned response

CS - conditioned stimulus

ERK - extracellular regulated kinases

FKBP51 - FK506-binding Protein 51

GABA - gamma-Aminobutyric acid

GSK3 $\alpha$ - glycogen synthase kinase 3 alpha

GSK3 $\beta$ - glycogen synthase kinase 3 beta

HPA axis -hypothalamic-pituitary-adrenal axis

LTD - long-term depression

LTP - long-term potentiation

MAPK - mitogen-activated protein kinases

NMDA - N-methyl-D-aspartate receptor

NTRK3 - neurotrophic tyrosine kinase, receptor, type 3

Nurr1 - nuclear receptor related 1 protein

$\mathrm{PI3K}$ - phosphoinositide 3-kinase

PP1 - protein phosphatase 1

pS9-GSK3 $\beta$ - phosphorylated GSK3 $\beta$ at Serine-9

PTSD - post-traumatic stress disorder

RNA - ribonucleic acid

SERT - serotonin transporter 
SNP - single-nucleotide polymorphism

SSRI - selective serotonin reuptake inhibitor

T2 - 3,5-diiodo-L-thyronine

US - unconditioned stimulus 


\section{Chapter 1. Introduction}

\section{Enhanced cognitive processing as a pathophysiological mechanism of major depressive disorder}

Major depression is epidemiologically spread, serious, recurrent disorder resulting in diminished quality of life and increased co-morbidities and mortality (Kessler \& Bromet, 2013). In the Western world, the rate of life prevalence of depressive disorder falls within a range of $8-23 \%$, with high numbers in the USA and Eastern Europe, including the Russian Federation (Garin et al., 2016; Margraf et al., 2016). All over the world, a problem of insufficient efficacy of treatment of depressed patients remains a significant medical and social challenge (Kessler et al., 2015).

The biological basis of depression remains to be not well understood that greatly complicates the development of new therapies of this medical condition. In particular, the augmented cognitive processing of adversities / negative experiences and memories for stress events are important in the pathophysiology of depression (Chrousos and Gold, 1992; Becker et al., 2008; Clark et al., 2009). Yet, the mechanisms of these processes and their relation to the formation of a depressive-like state are currently poorly investigated. Limited number of animal models was designed to address these phenomena. However, available data point to the role of enhanced acquisition of stress memories and adverse experiences as a highly relevant element of the development of depression, which is also involved in the mechanisms of other neuropsychiatric conditions, including the posttraumatic stress disorder (PTSD), generalized anxiety disorder, and phobias (Gold \& Korol, 2012, Flores et al., 2014). A better understanding of the neurobiology of these pathological processes could open new possibilities for therapeutical management of neuropsychiatric conditions associated with enhanced learning of adversities and particularly help to improve the treatment of depressed patients.

On one hand, an intact ability to acquire new memories, including learning of negative experiences, and successful storage / recall of these memories is 
a basic feature of adaptation and survival of the living organisms. On another hand, inappropriate retention of "emotionally negative" memories can be harmful and be involved in neuropsychiatric pathologies, including depression. Such memories can be intrusive and interfere with normal daily functioning, as it is a case with PTSD, where enhanced memorizing of adversities can lead to behavioural disruption, helplessness and feelings of worthlessness, resulting in symptoms of depression. Uncontrollable memories of the traumatic event can be distressing and aggravate depressive symptoms, that is also observed during phobias (Fig. 1; McNally et al., 1998; Yehuda et al., 2010; Wood et al., 2015). In case of depression and generalized anxiety disorder, it is the increase of processing of memories for negative / potentially threatening events that is prevalent in patients suffering from these conditions (Chrousos \& Gold, 1992). While a link with traumatizing event is not that obvious in the development of depression as it is with PTSD, it is believed that increased processing of negative experiences play an important role in the pathophysiology of depressive state (Siegmund \& Wotjak, 2006).

Contextual conditioning is believed to be a key mechanism of pathologically increased learning of adversities during neuropsychiatric conditions (Yehuda et al., 2007a; Yehuda \& LeDoux, 2007). A concept of contextual conditioning was proposed over a hundred years ago by Ivan Pavlov and upon the first publication in 1927 (Pavlov, 1927), has gain a broad acceptance worldwide, greatly impacting the fields of neurobiology, physiology, psychology and psychiatry. According to the concept of Pavlov, simultaneous activation of neuronal centers, which is caused by emotionally meaningful stimuli, e.g. pain, fear, nutritional stimulation or other biologically relevant factors, alone with a presentation of neutral elements, such as a context, at which emotional activation takes place, results in a formation of new functional links between the neuronal centers involved. As such, a representation of neutral sensory cues activates new networks and centers of emotional arousal mimicking the effects of the actual stimuli that caused a biological response in the previous pairing. 


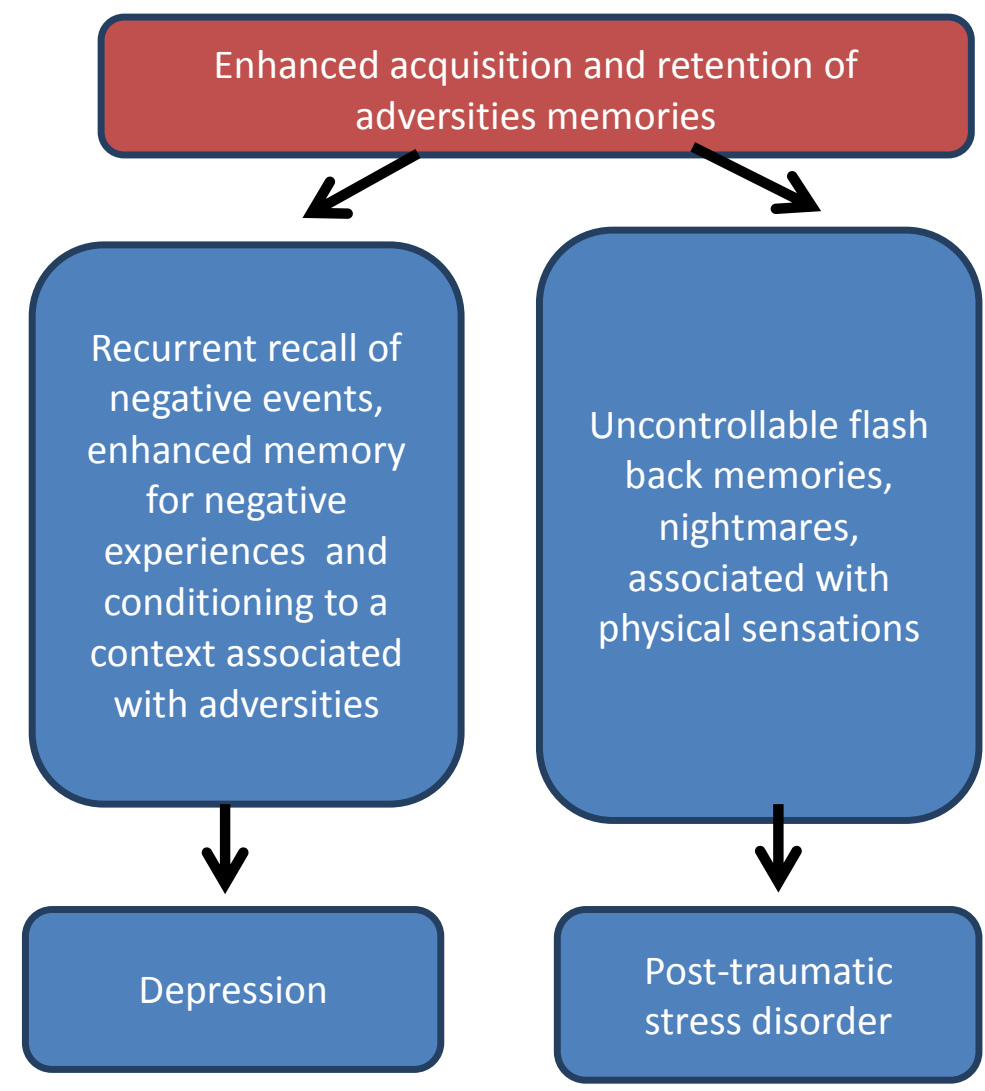

Figure 1. Pathologically enhanced learning of adversities results in the symptoms of depression and post-traumatic stress disorder. During depression and PTSD, excessive acquisition and retention of memories of adversities, where neutral factors such as elements of a context at which negative stimulation was experienced can lead to the development of specific symptoms of these disorders (adapted from Yehuda et al., 2010).

Thus, Pavlovian conditioning occurs when a conditioned stimulus (CS) is paired with an unconditioned stimulus (US) that is usually, of high biological potency and elicits the unconditioned response (UR). After pairing is repeated, the organism exhibits a conditioned response (CR) to the CS when the conditioned stimulus is presented alone. The CR is usually similar to the UR, but unlike the UR it must be acquired through experience and is relatively impermanent. The CS is not simply connected to the UR; and may differ from the UR. The developments of the concept of Pavlov during the subsequent decades have led to the formulation of the term "conditioned 
emotional response". This term is used to refer to the classic conditioning as a basis of emotional responses to contextual cues during such pathologies, as phobias and PTSD. The mechanisms of conditioned emotional response are thought to underlie not only these disorders but also depression, with some overlaps and differences (Fig. 1).

Although up to $50-60 \%$ of individuals experience a traumatic event in their life, only a minority develops psychiatric disorders. And still it is not clear why many remain resilient, other develop depression, and some suffer from PTSD or other diseases. It has been suggested that inter-individual differences in stress reaction and in coping behaviour with environmental challenges are crucial in determining the outcome after experience of a traumatic event (Koolhaas et al., 1999; Olff et al., 2005). Human and animal studies suggest distinct neural mechanisms to mediate coping behaviours under conditions of stressful events, such as passive strategy, an energy conserving mode of interaction, associated with high levels of denial or defensive emotion-inhibiting response, versus active coping strategy, that is based on the pro-active problem-focused coping style (De Boer et al., 1990; Strekalova, 1995; Keay \& Bandler, 2001; Olff et al., 2005). Over the recent years, a great deal of knowledge was accumulated regarding the neurobiology of individual susceptibility to stress and stress-related depressive syndrome (Krishnan et al., 2007; Strekalova et al., 2011; Drugan et al., 2013). Yet, very little is known about the mechanisms of interindividual differences in cognitive processing of traumatic / adverse experiences in relation to the development of a depressive-like state.

\section{Animal models of enhanced memories for adversities}

Most of the currently available rodent models of enhanced learning of adverse events were developed to study PTSD. They are based on the classic principle of Pavlovian conditioning and employ experimental settings in which animals learn to associate an aversive unconditioned stimulus, such as for example, a brief foot shock, air poof or bitter/aversive taste, with a neutral conditioned stimulus, typically a context, a tone, or a particular palatable taste (Maren, 2008). Perhaps, the most broadly applied model 
among this type of paradigms is the contextual fear conditioning paradigm, in which small rodents display so called "freezing behaviour". Freezing behaviour, a lack of mobility, is a species-specific form of expression of fear in rodents: immediate immobility in a situation of danger increases the chances for survival of small rodents in nature helping them to hide from a predator. In this paradigm, the duration of freezing behaviour displayed by rats and mice is taken as a measure of a vulnerability to the PTSD-like state (Anagnostaras et al., 2001; Maren \& Holt, 2004).

Enhanced memory of unpredictable, uncontrollable and inevitable aversive events can also result in learned helplessness, an important feature of depression, that can also be modelled in rodents (Anisman et al., 1983; Chourbaji et al., 2005). This feature implicates altered mechanisms of learning as well and, at least partly, is related to an increase of acquisition of negative associations by an individual (Pryce et al., 2011; Cryan \& Holmes, 2005). In comparison to other models, such as models of PTSD, the paradigms of learned helplessness mimic a deficit in coping with environmental challenges and involve instrumental forms of memory (Anisman et al., 1983). The models of learned helplessness originates from the phenomenon that an animal exposed to inescapable and uncontrollable electric shocks or other insults fails to flee the shocks or other adversities even when offered a means of escape (Anisman et al., 1983).

Indeed, the overlapping mechanisms between PTSD and learned helplessness are discussed in the current literature (Hammack et al., 2012). The fact that learned helplessness is dependent on the hippocampal formation, which is generally not considered to be the brain area underlying instrumental learning per se, speaks in favour for the existence of overlapping mechanisms between PTSD-associated changes in learning and generalized helplessness (Sheehan et al., 2003).

Helplessness in rodents is also considered to be manifested by floating behaviour in the forced swim test, a passive drifting in a water (Porsolt et al., 1977; Cryan \& Slattery, 2007; Huston et al., 2009), and immobility behaviour in the tail suspension model (Steru et al., 1987). These forms of behaviour are also regarded as an expression of emotional despair. With repeated 
forced swimming procedure, the elements of contextual learning were suggested to be involved in the expression of floating behaviour during forced swim test (Hawkins et al., 1978). The role of contextual cues of testing in induction of floating behaviour was supported in further studies (Stone et al., 2008). Evidence suggest that the antidepressant effects of imipramine and other compounds can be partly ascribed to their disruptive effects on memory formation (Cryan \& Holmes, 2005; see also below).

Yet, animal models that would let specifically investigate the neurobiology of contextual learning during the formation of a depressive-like state are not described in the literature. In a meantime, currently accumulated data summarized above, suggest that the mechanisms of helplessness, contextual learning and behavioural manifestation of emotional despair in a form of floating behaviour displayed by small rodents, are likely to interplay. This suggests a potential for the development of a paradigm of enhanced learning during a formation of a depressive-like state which could be based on these behavioural features in mice and rats.

\section{Neuroanatomical circuits of pathologically enhanced learning of adversities during depression}

Animal studies suggest that rodent behaviours in the paradigms of contextual fear conditioning, forced swimming and learned helplessness are regulated by the hippocampus, amygdala and the prefrontal cortex (Fig. 2; Hitchcock et al., 1989; Cisler et al., 2010; Browne et al., 2014). The overlap between the neuroanatomical substrates with these forms of behaviour further suggest a link between the mechanisms of plasticity and emotionality during enhanced learning of adversities associated with depression (Yang et al., 2012; Shishkina et al., 2012). While currently used models of fear conditioning, learned helplessness and behavioural despair are not designed to address specifically a phenomenon of enhanced acquisition of adversities during depression, they can provide useful information which is relevant for addressing the pathophysiology of elevated acquisition of adverse memories during depression. 


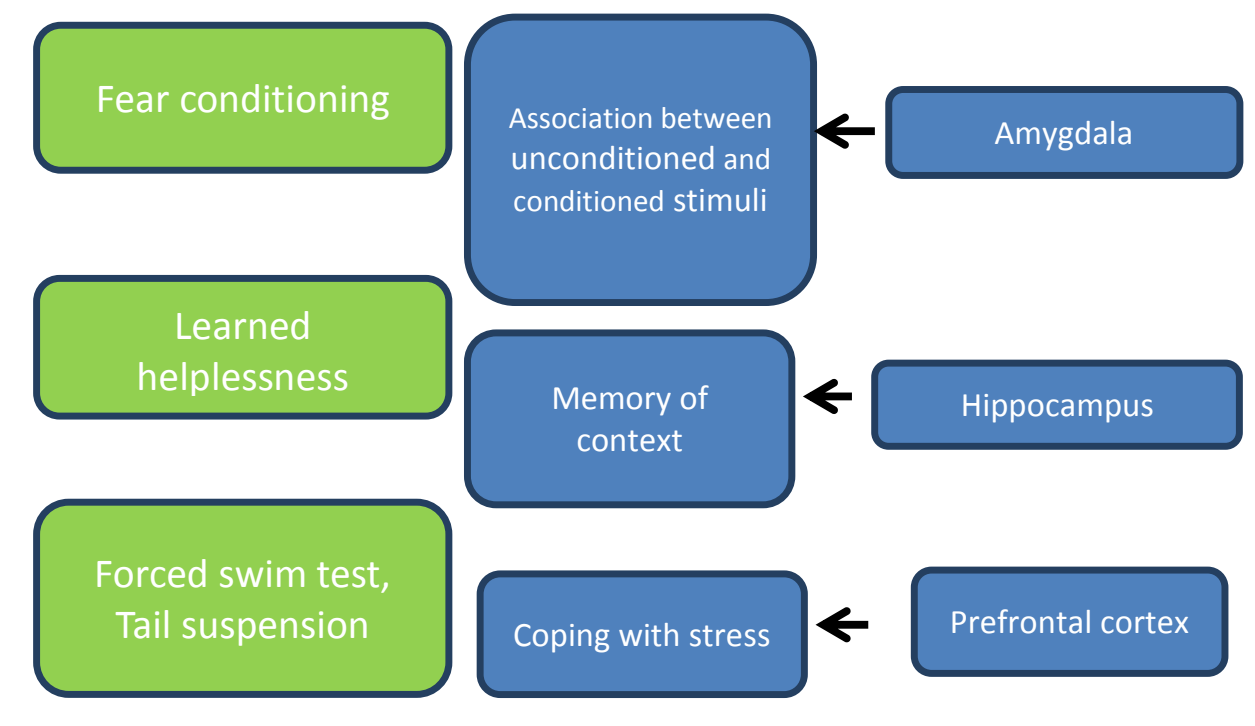

Figure 2. Neuroanatomical elements of enhanced learning/retention of adverse experiences in the fear conditioning, learned helplessness and forced swim tests. The overlap between the neuroanatomical substrates of fear conditioning, learned helplessness and forced swimming suggests a link between the mechanisms of plasticity and emotionality during enhanced learning of adversities associated with depression (adapted from Izquierdo et al., 2016).

Recently, Diamond and colleagues (2007) have proposed a model of neuroanatomical circuits of the formation of enhanced memory for adversities that are activated in a course of "temporal dynamics" of physiological response to stressful events. This concept is based on the view that during stressful situations, endogenous mechanisms of plasticity in the hippocampal formation and other elements of limbic structures are rapidly activated for a relatively short period of time and overlap with strong emotional arousal (Fig. 3). Under stress conditions, adverse events cause a release of cortisol and noradrenaline, which then stimulates long-term potentiation (LTP) and suppresses long-term depression (LTD), subsequently involving the $\mathrm{N}$-methyl-D-aspartate NMDA receptors and glycogen synthase kinase $3 \beta$ (GSK3 $\beta$ ) (Hawkins et al., 2006; Diamond et al., 2007; Peineau et al., 2007).

Apart from the hippocampus, another brain structure that is involved in memory acquisition, including contextual conditioning, is the prefrontal 
cortex (Gilmartin et al., 2014; Fig. 4). Besides contextual conditioning, the prefrontal cortex was shown to be critical for other forms of memory, which can be involved in enhanced cognitive processing during depression, including working memory, object recognition, other aspects of cognitive processing and decision-making (Diamond et al., 2007; Gold \& Korol, 2012). Importantly, the prefrontal cortex is regarded as crucial element of brain circuitry that regulates coping with the environmental challenges and stressors (Bland et al., 2003; Rangel et al., 2003; Amat et al., 2005).

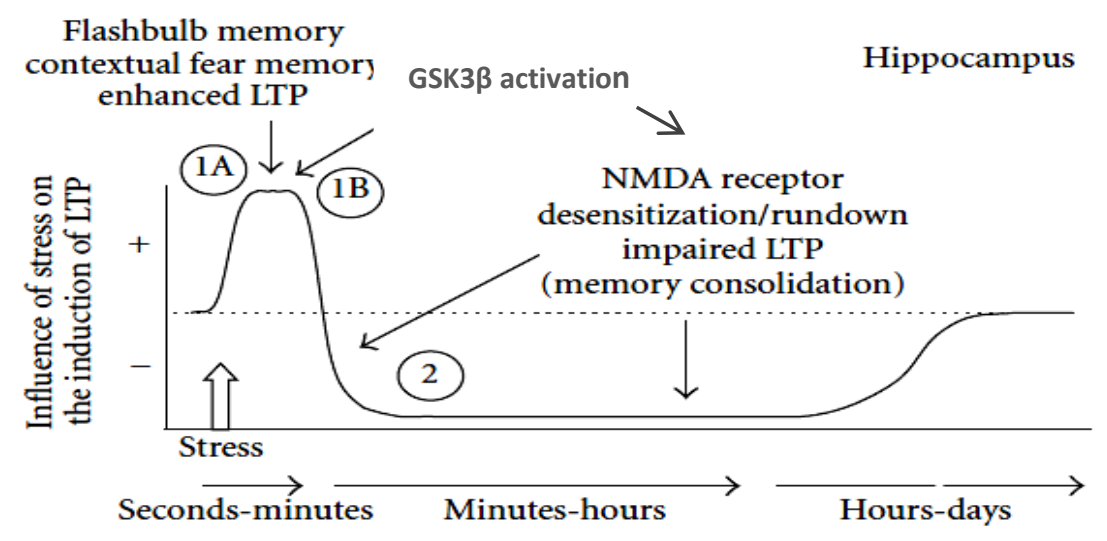

\section{Figure 3. Temporal dynamics of interactions between the elements of} stress and plasticity during enhanced learning of adverse events: the role of hippocampus. During abnormally increased acquisition of adverse / stressful memories, endogenous mechanisms of plasticity in the hippocampus and other elements of limbic structures are rapidly activated for a relatively short period of time and overlap with strong emotional arousal. The initiation of a strong emotional experience activates pathways of hippocampal neuroplasticity (phase 1). The initial phase (phase 1A), can implicate the secretion of adrenocorticotropic hormone, corticotropin releasing factor, and dopamine and be followed within minutes by release of glucocorticoids enhancement overexpression of GSK3. Within minutes of the initiation of the phase $1 \mathrm{~A}$, an enhancement of the long-term potentiation (phase $1 \mathrm{~B}$ ) takes place, that is followed by the Phase 2 , when the hippocampus undergoes a reversal of its plasticity state, which is mediated via a reduction in the sensitivity of NMDA receptors so the consolidation of acquired memory occurs (adapted from Diamond et al., 2007).

Different zones of this brain structure were shown to play distinct roles in stress response and memory. Ventral part of the prefrontal cortex was found to be involved in fear control in the rat, while the dorsal prefrontal cortex 
was reported to promote the expression of learned fear. Important function of the prefrontal cortex in the regulation of emotional control, in particular expression of fear, which is associated with previously acquired memories was demonstrated in numerous studies (Amat et al., 2005; Gilmartin et al., 2014).

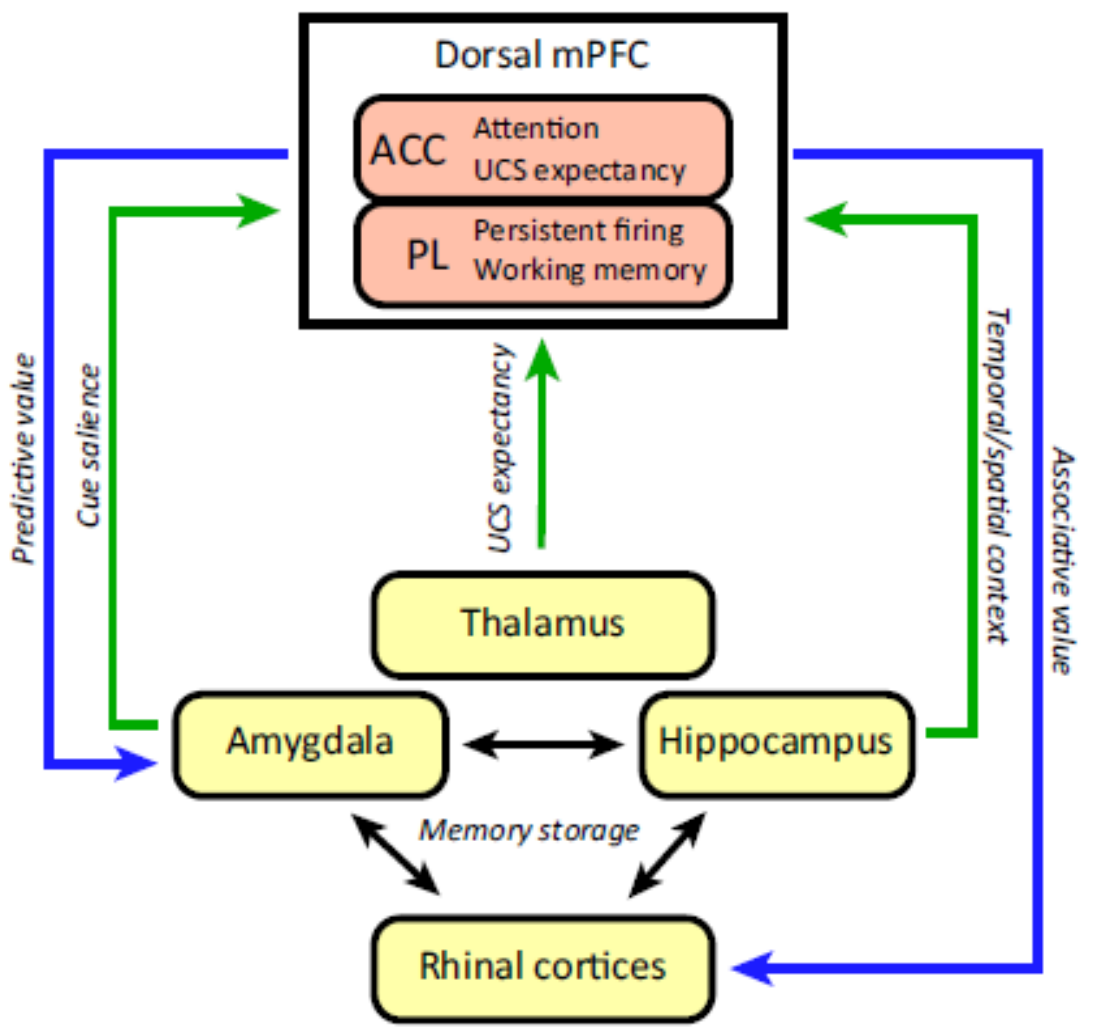

Figure 4. Pefrontal cortical regulation of the formation of fear memory. The dorsal prefrontal cortex integrates the information on adverse stimuli and context and regulates brain region activities which control attention, working and long-term memories, so complex fear associations are formed (adapted from Gilmartin et al., 2014).

Other brain structures were shown to be implemented in the mechanisms associated with enhanced learning of adversities and abnormal retention of negative experiences, such as the amygdala, thalamus and rhinal cortex (Fig 4). As for instance, the amygdala is known to regulate functions of the hippocampus during the acquisition of negative memories (Richardson et al., 
2004; Steinvorth et al., 2010). Imaging studies in humans revealed significant correlation between an activation of the amygdala and the hippocampus during learning of emotionally relevant stimuli (Dolcos et al., 2004; Kensinger \& Corkin, 2004). Electrical stimulation of the amygdala in rodents was found to mimic the emotion-induced enhancement of the hippocampal long-term potentiation (Frey et al., 2001; Akirav \& Richter-Levin, 2002). Also, the blockade of amygdala-hippocampal neuronal circuits resulted in a

blockade of the effects of stress on hippocampal long-term potentiation and contextual memory (Korz \& Frey, 2005); differential roles of these two brain structures in enhanced acquisition of adversities were suggested (Maren, 2008). The increases in intrinsic excitability in the amygdala during exposure to adverse experiences were shown to be related to its critical role in a convergence of conditional-unconditional stimulus, while hippocampus was found to play a key role in the conditioning and storage of these memories (An et al., 2016; Varela et al., 2016).

\section{Molecular and hormonal mechanisms of increased memory for adversities associated with depressive syndrome}

Taking into account crucial role of stress in the ethology of depression and PTSD, it might be anticipated that neuroendocrine changes underlying stress response, such as secretion of glucocorticoids, noradrenaline and overactivation of the hypothalamic-pituitary-adrenal (HPA) axis can be the key regulators of enhanced retention of adverse memories (Fig. 5). Human studies support this view. As for instance, a study of Kuhlmann and Wolf (2006) have shown that healthy volunteers treated with cortisol display a better memory for aversive pictures (Kuhlmann \& Wolf, 2006; Oliver \& Wolf, 2009).

During formation of enhanced memories for negative events, increased hormonal and physiological response to stress takes place in parallel with molecular changes underlying memory formation. As such, molecular crosstalk between these processes is thought to underlie the mechanisms of increased consolidation and retention of adverse memories (Diamond et al., 2007; Gazzaniga et al., 2014). Among the four steps of memory formation, 
the acquisition, consolidation of memory, memory storage and retrieval, the first two are involved in the processes of enhanced learning of adversities as they occur simultaneously with the stress event (Hawkins et al., 2006; Peineau et al., 2007). These two stages of learning are likely to be affected, dependently on individual features of stress, which are largely determined by the expression of mineralocorticoid and glucocorticoid receptors in the CNS (Reul \& de Kloet, 1985; Wingenfeld \& Wolf, 2014; De Kloet \& Molendijk, 2016).

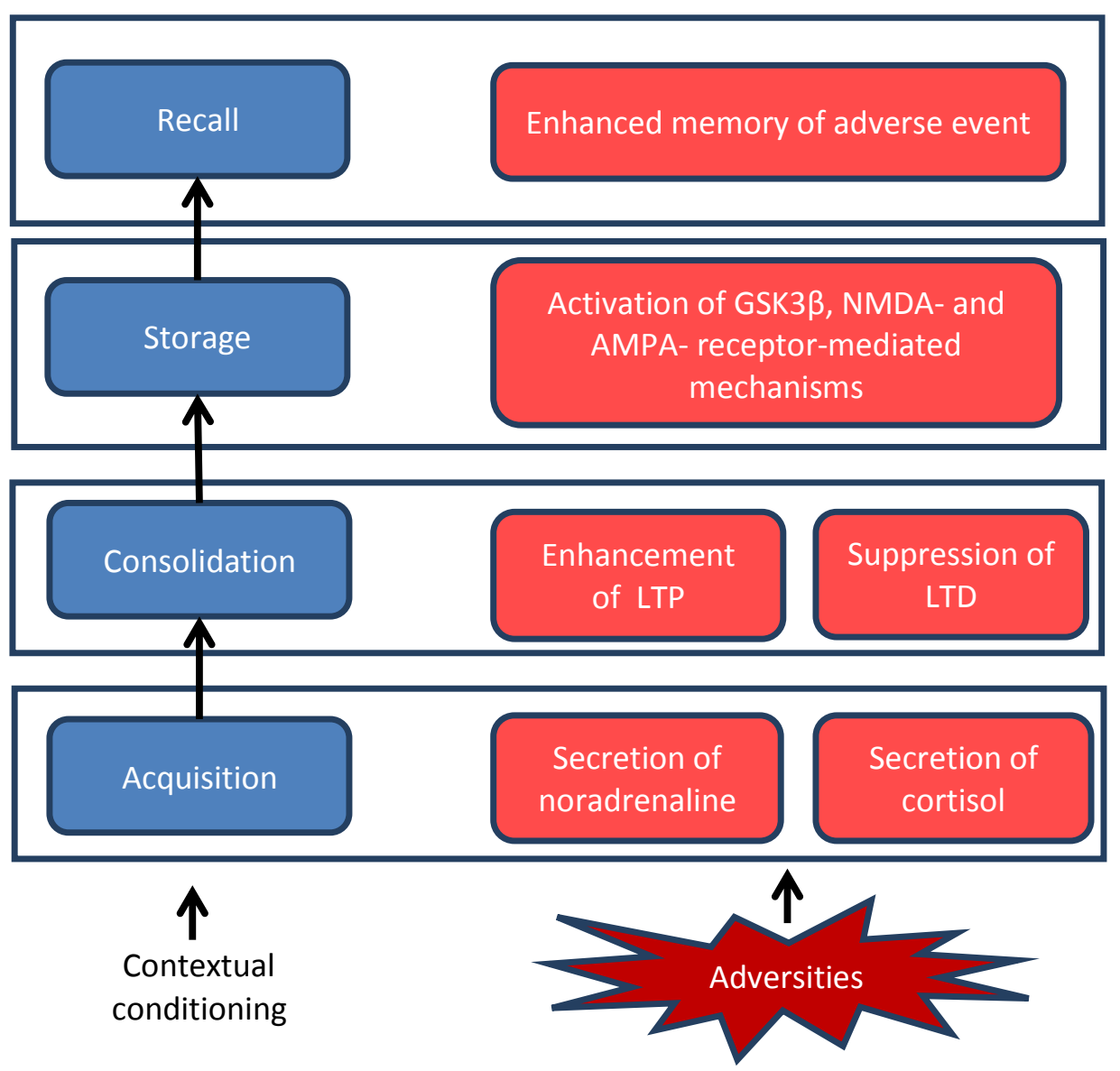

Figure 5. Hormonal and neurophysiological events and enhanced acquisition of adverse memories. Stages of memory formation and hormonal / molecular changes associated with stressful experiencing of adversities (adapted from Morin et al., 2015). 
While depression is associated with reduced response to glucocorticoids and increased cortisol levels (Lupien et al., 1999; Sapolsky et al., 2000; O'Brien et al., 2004), patients with PTSD display an opposing alternation (Rohleder et al., 2004; Yehuda, 2009). Increased responsiveness to glucocorticoids in PTSD patients is associated with enhanced sensitivity of glucocorticoid receptors, as well as a reduction in cortisol levels (Yehuda et al., 2007; Yehuda \& LeDoux, 2007). These hormonal differences in stress-related regulation may underlie distinct characters of pathophysiological enhanced memory during major depression versus PTSD. While PTSD patients typically complain to "flashback memories", the major depression is associated with recurrent recalls on negative experiences and avoidance of associated with this experience context.

Other stress hormones can play an important role in the mechanisms of enhanced retention of stressful and adverse events during depression. As for instance, an interaction between the secretion of cortisol and noradrenaline during the acquisition of negative information was found to be such factor (Oliver \& Wolf, 2009). A blockade of noradrenaline receptors with propranolol have disrupted amygdala activation in subjects with a high endogenous cortisol levels and reduced learning of adverse events (Cahill et al., 1994). Studies with healthy subjects (Lupien et al., 1999) and PTSD patients (Reist et al., 2001) showed that a recall of an aversive event involves the activation of central, but not peripheral, $\beta$-adrenergic receptors. These data further support the hypothesis that high secretion of cortisol at the time of acquisition of aversive information, including a context, can interact with the noradrenergic activation in the limbic system, and thus, regulate learning of adversities (van Stegeren, 2008).

\section{GSK3 $\beta$ and molecular mechanisms of depression and neuronal plasticity}

A body of data has established the role of glycogen synthase kinase 3 (GSK3) in depression, stress response and brain plasticity. Given that this molecule, on one hand, regulates both stress response and involved in the pathophysiology of depression, and on another hand, is critical for the mechanisms of brain plasticity, it was suggested to underlie pathologically 
enhanced learning of adversities (Beurel et al., 2015). GSK3 is a multifunctional serine/threonine kinase that was originally identified as a highly specific for glycogen synthase regulator of glycogen metabolism (Embi et al., 1980). Ubiquitously expressed in eukaryotes (Jope \& Roh, 2006), GSK3 plays a fundamental role in a wide variety of functions, including cell division, proliferation, differentiation, and adhesion (Takahashi et al., 1994; Peineau et al., 2007; Peineau et al., 2008; Beurel et al., 2015). Among two known isoforms of GSK3 in mammals, GSK3 $\alpha$ and GSK3 $\beta$ (Kaidanovich-Beilin \& Woodgett, 2011), GSK3 $\beta$ is highly expressed in numerous structures of the brain, particularly in the hippocampus (Graef et al., 1999; Leroy \& Brion, 1999). The inhibition of GSK3 $\beta$ by phosphorylation at serine-9 is considered to be the main regulatory mechanism of GSK3 $\beta$ activity (Polter et al., 2010).

Elevated levels of GSK3 $\beta$ and increased activity of this kinase are wellestablished to be associated with depression and distress (Beurel et al., 2015). Mutant mice over-expressing GSK3 $\beta$ exhibit increased sensitivity to stress-induced depression-like behaviours (Beurel et al., 2015). Depressive disease is well-known to be sensitive to the therapeutics that inhibit GSK3 $\beta$. Patients with depression respond to therapies with mood stabilizer lithium, which directly binds and inhibits GSK3 $\beta$ by competing for a $\mathrm{Mg}^{2+}$ binding site (Klein \& Melton, 1996; Stambolic et al., 1996). The administration of lithium at therapeutically relevant concentrations also increases the phosphorylation of GSK3 at serine 9 that inhibits the activity of this kinase (Jope \& Johnson, 2004; Li et al., 2007). Additionally, in mice displaying elevated signs of learned helplessness, diminished levels of the serinephosphorylated GSK3 $\beta$ were found (Polter et al., 2010).

Apart from its role in stress and depressive-like mechanisms, GSK3 $\beta$ is critical for hippocampal plasticity. A lack of GSK3 $\beta$ activity prevents a formation of hippocampal LTP. Interestingly, Kimura and colleagues (Kimura et al., 2008) have found that the inhibition of GSK3 $\beta$ before the fear conditioning did not impair memory consolidation, but inhibition of GSK3 $\beta$ before the reexposure to context of stress significantly impaired memory in this paradigm. Thus, GSK3 $\beta$ is likely involved in consolidation phase of learning of adverse memories, rather than in the acquisition phase. 
The functions of GSK3 $\beta$ as an important regulator of hippocampal plasticity are associated with NMDA receptor- mediated mechanisms (Nader et al., 2000; Peineau et al., 2007, 2008, 2009). In particular, it was reported (Kimura et al., 2008) that the inhibition of GSK3 $\beta$ prevents the induction of NMDA-dependent LTD and modulate a balance between LTP and LTD (Fig. $6)$.

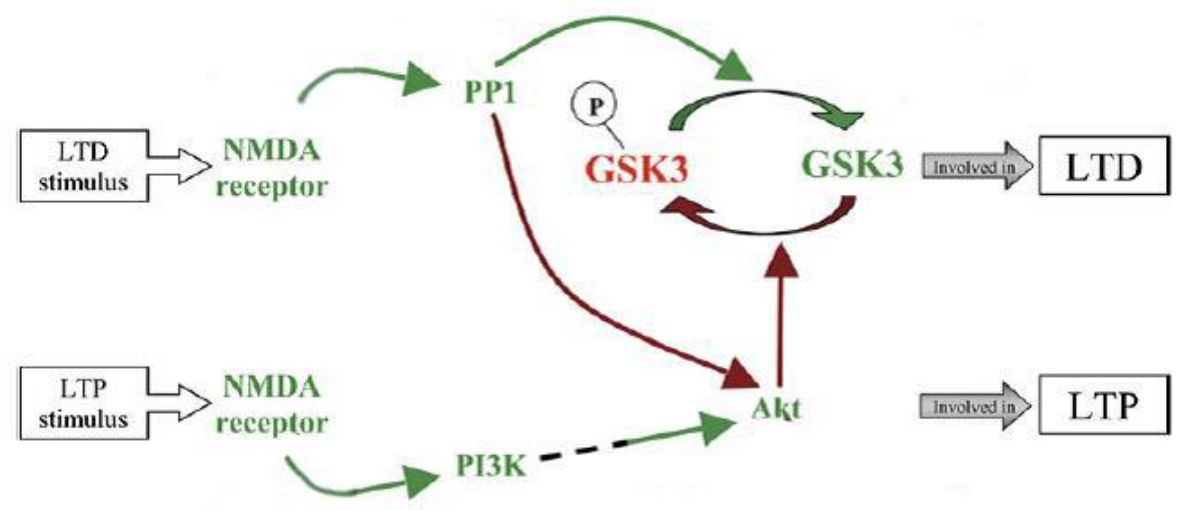

Figure 6. GSK3 $\beta$ regulates NMDA-dependent synaptic plasticity. The level of activity of GSK3 $\beta$ determines whether NMDA receptor activation induces long-term potentiation (LTP) or inhibits long-term-depression (LTD) in the hippocampus. During longterm-depression, activation of protein phosphatase 1 (PP1) leads to dephosphorylation of GSK3 $\beta$ at serine-9 to activation of GSK3 $\beta$ and results in LTD to occur. PP1 also inhibits Protein kinase $B$ (Akt). During LTP, activation of NMDA receptors leads to stimulation of the Phosphoinositide 3-kinase- Protein kinase B (PI3K-Akt) pathway, which phosphorylates GSK3 $\beta$ at serine-9, and prevent the induction of LTD (adapted from Kimura et al., 2008).

Recent data have further suggested the role of GSK3 $\beta$ in the pathways of enhanced acquisition of negative experiences during PTSD and depression (Chen et al., 2014). As for instance, it was shown that long-term memories for contextual fear, a paradigm of PTSD-like state, were associated with decreased levels of phosphorylated (inactive) form of GSK3 $\beta$ in the hippocampus (Dahlhoff et al., 2010). These and other results (Chew et al., 2015; Kimura et al., 2008; Shi et al., 2014) suggest the role of GSK3 $\beta$ in 
pathophysiological enhanced learning of adverse context during neuropsychiatric conditions. Yet, no specific experiments that would address the role of GSK3 $\beta$ in the mechanisms of increased contextual memory for negative events during depression were conducted, according to available literature.

Forced swim and tail suspension tests as paradigms of depressive behaviour: implications for modeling of pathologically enhanced cognitive processing

The forced swim test (Porsolt, 1977) and the tail suspension test (Steru et al., 1985) are broadly used animal models of depression (Lucki, 1997; Cryan \& Mombereau, 2004). These tests are based on the induction and scoring of floating or immobility, respectively, which rodents naturally display being placed in an inescapable situation. While these paradigms are probably the most commonly and frequently used models in basic and applied research on depression, there are still debates about their validity and the interpretation of floating and immobility behaviours which are displayed by the rodents in the tests (McArthur \& Borsini, 2006; Harro et al., 2011). These two tests for depressive-like behaviour were shown to have limited pharmacological sensitivity. A testing of antidepressant compounds with their use was reported to result in false positive/negative outcomes. Besides, there are differences between these tests in sensitivity to drugs reported in previous works, different strains of rodents do not exhibit similar changes in floating/immobility. As for instance, Ampuero and colleagues (2015) reported that in rats exposed to a restraint stress, a treatment with antidepressant reboxetine has reduced the immobility scores in the forced swim test, but not in the tail suspension test. It was suggested that hypothermia could have a factor that interferes on drug activity and can be a reason of discrepant outcomes from two models. Of interest, in one of these studies, antidepressant effects of treatment with desipramine were detectable only if mice were tested in $30^{\circ} \mathrm{C}$ water temperature but not at $25^{\circ} \mathrm{C}$ (Bogdanova, et al., 2013). 
An exposure of experimental animals to the forced swim or tail suspension stressor induces a profound response of the sympathetic nervous system, an over-activation of the HPA axis, and a release of a variety of neurotransmitters including dopamine, serotonin, gamma-aminobutyric acid (GABA) in the brain (Cryan \& Slattery, 2007; Bogdanova et al., 2013). Hormonal response of HPA axis was shown to be interrelated with floating behaviour in the forced swim test (Cabib \& Puglisi-Allegra, 2012). As it was mentioned above, the corticosteroids can mediate coping with stress; their release is altered during the forced swim test. With an exposure to the forced swimming, the mineralocorticoid receptors in the hippocampus were shown to be up-regulated $8 \mathrm{~h}$ thereafter at gene level, and $24 \mathrm{~h}$ thereafter, at the protein level, as shown with the radioligand binding (De Kloet \& Molendijk, 2016). The administration of spironolactone, mineralocorticoid receptors antagonist, has reduced floating behaviour (De Kloet \& Molendijk, 2016). An activation of glucocorticoid receptors by the administration of dexamethasone immediately after the first swim session has dosedependently increased the duration of previously acquired floating behaviour during repeated testing that was carried out $24 \mathrm{~h}$ after the initial swim session (De Kloet \& Molendijk, 2016).

As it was discussed previously, besides the elements of HPA axis, repeated tests for the floating/immobility behaviour involve the activation of pathways of learning and memory. For example, it was demonstrated that cycloheximide and anisomycin, well-known blockers of protein synthesis and molecular cascades of memory, can reduce immobility in the forced swim test (De Pablo et al., 1989; Bogdanova et al., 2013). From that, a combination of repeated exposures to forced swimming / tail suspension could be used in modeling of learning of adverse context that is associated with a depressive state.

In addition, as it was summarized above, individual differences in hormonal stress response are regarded to be associated with passive and active coping strategies (De Boer et al., 1990), and physiological features of "personality traits" are related to a susceptibility to depression (Lesch, 2011; Weber et al., 2011). Floating and immobility behaviours are considered by some 
authors as a sign of passive coping strategy of animals (Steru et al., 1985; Warden et al., 2012). Furthermore, a group of Cabib and Puglisi-Allegra (2012) showed that enhanced tonic mesoaccumbens dopamine activity parallels the expression of active stress-induced coping styles, while inhibition of dopamine release is required for passive coping in the forced swim tests.

To sum up, the paradigms of forced swimming and tail suspension, when applied in repeated manner, can combine the features of depression models, as well as be paradigms of contextual memory. From that, under certain conditions, such as repeated use on the same animal, they can be validated as potential models of enhanced memory processing associated with the formation of a depressive-like state.

\section{Hypothesis and aim}

In our work, first, we studied potential antidepressant-like effects of thyroid hormone $\mathrm{T} 2$ in mice that were suggested by gene expression studies in the model of stress-induced anhedonia (Chapter 2). Antidepressant-like effects of $\mathrm{T} 2$ were found by us in mice tested in classic version of the tail suspension model, but not of the forced swim test (Chapter 2, Chapter 3). This discrepancy prompted us to undertake such modifications of the mouse forced swim test that would increase its accuracy and sensitivity. We hypothesized that an additional swim session occurring on Day 5 following the initial exposure in this test could model a phase of "consolidation" of neurobiological trace of a depressive-like state, associated, in particular, with the mechanisms of enhanced cognitive processing.

We found that, indeed, additional delayed exposure of mice to swim session resulted to a potentiation of floating behaviour, which was observed during the first minutes of testing (Chapter 4 ). This change was abolished by low dose of imipramine which was ineffective during classic variants of swim test (Chapter 4); this finding was later supported by similar experiment with antidepressant treatment (Chapter 5). Our findings suggested physiological importance of a pause between repeated testing and prompted us to study 
molecular mechanisms of this phenomenon. Since GSK3 $\beta$ is a wellestablished factor of both depression and memory, we studied the changes of expression of this molecule both on gene and protein levels, in the hippocampus and prefrontal cortex that are involved in these processes. We found that GSK3 $\beta$ is over-expressed specifically during delayed additional testing in the forced swim test in hippocampus of all mice and in the prefrontal cortex of individuals that display increased depressive-like behaviour (Chapter 4). Also, our experiments have demonstrated a key role of context and timing in the development of depressive behaviour in our model. Separate studies were devoted to the question whether antidepressant treatments with compounds of different classes that suppress GSK3 $\beta$ can interfere with depressive-like changes in the modified swim test. We have shown that thiamine (vitamin B1) and its precursor benfotiamine, as well as imipramine, prevent a potentiation of floating and brain over-expression of GSK3 $\beta$ in the modified swim test (Chapter 5). These results further supported our conclusion as for the role of GSK3 $\beta$ in enhanced cognitive processing during depressive state.

\section{References}

Akirav, I., Richter-Levin, G., 2002. Mechanisms of amygdala modulation of hippocampal plasticity. J. Neurosci. 22, 9912-21.

Amat, J., Baratta, M. V, Paul, E., Bland, S.T., Watkins, L.R., Maier, S.F., 2005. Medial prefrontal cortex determines how stressor controllability affects behaviour and dorsal raphe nucleus. Nat. Neurosci. 8, 365-71.

Ampuero, E., Luarte, A., Santibañez, M., Varas-Godoy, M., Toledo, J., DiazVeliz, G., Cavada, G., Rubio, F.J., Wyneken, U., 2015. Two Chronic Stress Models Based on Movement Restriction in Rats Respond Selectively to Antidepressant Drugs: Aldolase C As a Potential Biomarker. Int. J. Neuropsychopharmacol. 18, 38-42.

An, Y., Chen, C., Inoue, T., Nakagawa, S., Kitaichi, Y., Wang, C., Izumi, T., Kusumi, I., 2016. Mirtazapine exerts an anxiolytic-like effect through activation of the median raphe nucleus-dorsal hippocampal 5-HT pathway in contextual fear conditioning in rats. Prog. NeuroPsychopharmacology Biol. Psychiatry 70, 17-23. 
Anagnostaras, S.G., Gale, G.D., Fanselow, M.S., 2001. Hippocampus and contextual fear conditioning: recent controversies and advances. Hippocampus 11, 8-17.

Anisman, H., Irwin, J., Beauchamp, C., Zacharko, R., 1983. Cross-stressor immunization against the behavioural deficits introduced by uncontrollable shock. Behav. Neurosci. 97, 452-61.

Bai, F., Li, X., Clay, M., Lindstrom, T., Skolnick, P., n.d. Intra- and interstrain differences in models of "behavioural despair". Pharmacol. Biochem. Behav. 70, 187-92.

Becker, C., Zeau, B., Rivat, C., Blugeot, A., Hamon, M., Benoliel, J.-J., 2008. Repeated social defeat-induced depression-like behavioural and biological alterations in rats: involvement of cholecystokinin. Mol. Psychiatry 13, 1079-92.

Beurel, E., Grieco, S.F., Jope, R.S., 2015. Glycogen synthase kinase-3 (GSK3): Regulation, actions, and diseases. Pharmacol. Ther. 148, 114-31.

Bland, S.T., Hargrave, D., Pepin, J.L., Amat, J., Watkins, L.R., Maier, S.F., 2003. Stressor controllability modulates stress-induced dopamine and serotonin efflux and morphine-induced serotonin efflux in the medial prefrontal cortex. Neuropsychopharmacology 28, 1589-96.

Bogdanova, O. V., Kanekar, S., D’Anci, K.E., Renshaw, P.F., 2013. Factors influencing behaviour in the forced swim test. Physiol. Behav. 118, 22739.

Cabib, S., Puglisi-Allegra, S., 2012. The mesoaccumbens dopamine in coping with stress. Neurosci. Biobehav. Rev. 36, 79-89.

Cahill, L., Prins, B., Weber, M., McGaugh, J.L., 1994. Beta-adrenergic activation and memory for emotional events. Nature 371, 702-4.

Chen, C., Ji, M., Xu, Q., Zhang, Y., Sun, Q., Liu, J., ... Li, W. (2014). Sevoflurane attenuates stress-enhanced fear learning by regulating hippocampal BDNF expression and Akt/GSK3 $\beta$ signaling pathway in a rat model of post-traumatic stress disorder. Journal of Anesthesia, 600-8.

Chew, B., Ryu, J. R., Ng, T., Ma, D., Dasgupta, A., Neo, S. H., ... Goh, E. L. K. (2015). Lentiviral silencing of GSK3 $\beta$ in adult dentate gyrus impairs contextual fear memory and synaptic plasticity. Frontiers in Behavioural 
Neuroscience, 9, 158.

Chourbaji, S., Zacher, C., Sanchis-Segura, C., Dormann, C., Vollmayr, B., Gass, P., 2005. Learned helplessness: validity and reliability of depressive-like states in mice. Brain Res. Brain Res. Protoc. 16, 70-8.

Chrousos, G.P., Gold, P.W., 1992. The concepts of stress and stress system disorders. Overview of physical and behavioural homeostasis. JAMA 267, 1244-52.

Clark, L., Chamberlain, S.R., Sahakian, B.J., 2009. Neurocognitive mechanisms in depression: implications for treatment. Annu. Rev. Neurosci. 32, 5774.

Cryan, J.F., Holmes, A., 2005. The ascent of mouse: advances in modelling human depression and anxiety. Nat. Rev. Drug Discov. 4, 775-90.

Cryan, J.F., Mombereau, C., 2004. In search of a depressed mouse: utility of models for studying depression-related behaviour in genetically modified mice. Mol. Psychiatry 9, 326-57.

Cryan, J.F., Slattery, D.A., 2007. Animal models of mood disorders: Recent developments. Curr. Opin. Psychiatry 20, 1-7.

De Boer, S.F., Slangen, J.L., Van der Gugten, J., 1990. Plasma catecholamine and corticosterone levels during active and passive shock-prod avoidance behaviour in rats: Effects of chlordiazepoxide. Physiol. Behav. 47, 1089-1098.

De Kloet, E.R., Molendijk, M.L., 2016. Coping with the Forced Swim Stressor: Towards Understanding an Adaptive Mechanism. Neural Plast. 2016, 465-478.

De Pablo, J.M., Parra, A., Segovia, S., Guillamón, A., 1989. Learned immobility explains the behaviour of rats in the forced swimming test. Physiol. Behav. 46, 229-37.

Diamond, D.M., Campbell, A.M., Park, C.R., Halonen, J., Zoladz, P.R., 2007. The temporal dynamics model of emotional memory processing: $A$ synthesis on the neurobiological basis of stress-induced amnesia, flashbulb and traumatic memories, and the Yerkes-Dodson law. Neural Plast. 2007, 1-33. 
Dolcos, F., LaBar, K.S., Cabeza, R., 2004. Interaction between the amygdala and the medial temporal lobe memory system predicts better memory for emotional events. Neuron 42, 855-63.

Drugan, R.C., Christianson, J.P., Warner, T.A., Kent, S., 2013. Resilience in shock and swim stress models of depression. Front. Behav. Neurosci. 7, 14.

Embi, N., Rylatt, D.B., Cohen, P., 1980. Glycogen synthase kinase-3 from rabbit skeletal muscle. Separation from cyclic-AMP-dependent protein kinase and phosphorylase kinase. Eur. J. Biochem. 107, 519-27.

Flores, Á., Valls-Comamala, V., Costa, G., Saravia, R., Maldonado, R., Berrendero, F., 2014. The hypocretin/orexin system mediates the extinction of fear memories. Neuropsychopharmacology 39, 2732-41.

Frey, S., Bergado-Rosado, J., Seidenbecher, T., Pape, H.C., Frey, J.U., 2001. Reinforcement of early long-term potentiation (early-LTP) in dentate gyrus by stimulation of the basolateral amygdala: heterosynaptic induction mechanisms of late-LTP. J. Neurosci. 21, 3697-703.

Garin, N., Koyanagi, A., Chatterji, S., Tyrovolas, S., Olaya, B., Leonardi, M., Lara, E., Koskinen, S., Tobiasz-Adamczyk, B., Ayuso-Mateos, J.L., Haro, J.M., 2016. Global Multimorbidity Patterns: A Cross-Sectional, Population-Based, Multi-Country Study. Journals Gerontol. - Ser. A Biol. Sci. Med. Sci. 71, 205-14.

Gazzaniga, Michael S., Richard B. Ivry, and G.R.M., 2014. Chapter 9: Memory. Cogn. Neurosci. Biol. Mind. 4th ed 378-423.

Gilmartin, M.R., Balderston, N.L., Helmstetter, F.J., 2014. Prefrontal cortical regulation of fear learning. Trends Neurosci. 37, 445-64.

Gold, P.E., Korol, D.L., 2012. Making memories matter. Front. Integr. Neurosci. 6, 116.

Graef, I.A., Mermelstein, P.G., Stankunas, K., Neilson, J.R., Deisseroth, K., Tsien, R.W., Crabtree, G.R., 1999. L-type calcium channels and GSK-3 regulate the activity of NF-ATc4 in hippocampal neurons. Nature 401, 703-8.

Hammack, S.E., Cooper, M.A., Lezak, K.R., 2012. Overlapping neurobiology of learned helplessness and conditioned defeat: Implications for PTSD and 
mood disorders. Neuropharmacology 62, 565-75.

Harro, J., Kanarik, M., Matrov, D., Panksepp, J., 2011. Mapping patterns of depression-related brain regions with cytochrome oxidase histochemistry: relevance of animal affective systems to human disorders, with a focus on resilience to adverse events. Neurosci. Biobehav. Rev. 35, 1876-89.

Hawkins, J., Hicks, R.A., Phillips, N., Moore, J.D., 1978. Swimming rats and human depression. Nature 274, 512-3.

Hawkins, R.D., Kandel, E.R., Bailey, C.H., 2006. Molecular mechanisms of memory storage in Aplysia. Biol. Bull. 210, 174-91.

Huston, J.P., Schulz, D., Topic, B., 2009. Toward an animal model of extinction-induced despair: focus on aging and physiological indices. J. Neural Transm. 116, 1029-36.

Izquierdo, I., Furini, C.R.G., Myskiw, J.C., 2016. Fear Memory. Physiol. Rev. 96, 695 LP-750.

Jope, R.S., Johnson, G.V.W., 2004. The glamour and gloom of glycogen synthase kinase-3. Trends Biochem. Sci. 29, 95-102.

Jope, R.S., Roh, M.-S., 2006. Glycogen synthase kinase-3 (GSK3) in psychiatric diseases and therapeutic interventions. Curr. Drug Targets 7, 1421-34.

Kaidanovich-Beilin, O., Woodgett, J.R., 2011. GSK-3: Functional Insights from Cell Biology and Animal Models. Front. Mol. Neurosci. 4, 40.

Keay, K.A., Bandler, R., 2001. Parallel circuits mediating distinct emotional coping reactions to different types of stress. Neurosci. Biobehav. Rev. $25,669-678$.

Kensinger, E.A., Corkin, S., 2004. Two routes to emotional memory: distinct neural processes for valence and arousal. Proc. Natl. Acad. Sci. U. S. A. 101, 3310-5.

Kessler, R.C., Bromet, E.J., 2013. The epidemiology of depression across cultures. Annu. Rev. Public Health 34, 119-38.

Kessler, R.C., Sampson, N.A., Berglund, P., Gruber, M.J., Al-Hamzawi, A., Andrade, L., Bunting, B., Demyttenaere, K., Florescu, S., de Girolamo, G., Gureje, O., He, Y., Hu, C., Huang, Y., Karam, E., Kovess-Masfety, V., Lee, 
S., Levinson, D., Medina Mora, M.E., Moskalewicz, J., Nakamura, Y., Navarro-Mateu, F., Browne, M.A.O., Piazza, M., Posada-Villa, J., Slade, T., Ten Have, M., Torres, Y., Vilagut, G., Xavier, M., Zarkov, Z., Shahly, V., Wilcox, M.A., 2015. Anxious and non-anxious major depressive disorder in the World Health Organization World Mental Health Surveys. Epidemiol. Psychiatr. Sci. 24, 210-26.

Kimura, T., Yamashita, S., Nakao, S., Park, J.M., Murayama, M., Mizoroki, T., Yoshiike, Y., Sahara, N., Takashima, A., 2008. GSK3 $\beta$ is required for memory reconsolidation in adult brain. PLoS One, 3, 16.

Klein, P.S., Melton, D.A., 1996. A molecular mechanism for the effect of lithium on development. Proc. Natl. Acad. Sci. U. S. A. 93, 8455-9.

Korz, V., Frey, J.U., 2005. Bidirectional modulation of hippocampal long-term potentiation under stress and no-stress conditions in basolateral amygdala-lesioned and intact rats. J. Neurosci. 25, 7393-400.

Krishnan, V., Han, M.H., Graham, D.L., Berton, O., Renthal, W., Russo, S.J., LaPlant, Q., Graham, A., Lutter, M., Lagace, D.C., Ghose, S., Reister, R., Tannous, P., Green, T.A., Neve, R.L., Chakravarty, S., Kumar, A., Eisch, A.J., Self, D.W., Lee, F.S., Tamminga, C.A., Cooper, D.C., Gershenfeld, H.K., Nestler, E.J., 2007. Molecular Adaptations Underlying Susceptibility and Resistance to Social Defeat in Brain Reward Regions. Cell 131, 391-404.

Kuhlmann, S., Wolf, O.T., 2006. Arousal and cortisol interact in modulating memory consolidation in healthy young men. Behav. Neurosci. 120, 217-23.

Leroy, K., Brion, J.P., 1999. Developmental expression and localization of glycogen synthase kinase-3beta in rat brain. J. Chem. Neuroanat. 16, 279-93.

Lesch, K.-P., 2011. When the serotonin transporter gene meets adversity: the contribution of animal models to understanding epigenetic mechanisms in affective disorders and resilience. Curr. Top. Behav. Neurosci. 7, 251-80.

Li, X., Rosborough, K.M., Friedman, A.B., Zhu, W., Roth, K.A., 2007. Regulation of mouse brain glycogen synthase kinase-3 by atypical antipsychotics. Int. J. Neuropsychopharmacol. 10, 7-19. 
Lucki, I., 1997. The forced swimming test as a model for core and component behavioural effects of antidepressant drugs. Behav. Pharmacol. 8, 52332.

Lupien, S.J., Nair, N.P., Brière, S., Maheu, F., Tu, M.T., Lemay, M., McEwen, B.S., Meaney, M.J., 1999. Increased cortisol levels and impaired cognition in human aging: implication for depression and dementia in later life. Rev. Neurosci. 10, 117-39.

Maier, S.F., Watkins, L.R., 2005. Stressor controllability and learned helplessness: the roles of the dorsal raphe nucleus, serotonin, and corticotropin-releasing factor. Neurosci. Biobehav. Rev. 29, 829-41.

Maren, S., 2008. Pavlovian fear conditioning as a behavioural assay for hippocampus and amygdala function: cautions and caveats. Eur. J. Neurosci. 28, 1661-6.

Maren, S., Holt, W.G., 2004. Hippocampus and Pavlovian fear conditioning in rats: muscimol infusions into the ventral, but not dorsal, hippocampus impair the acquisition of conditional freezing to an auditory conditional stimulus. Behav. Neurosci. 118, 97-110.

Margraf, J., Lavallee, K., Zhang, X., Schneider, S., 2016. Social Rhythm and Mental Health: A Cross-Cultural Comparison. PLoS One 11, e0150312.

McArthur, R., Borsini, F., 2006. Animal models of depression in drug discovery: a historical perspective. Pharmacol. Biochem. Behav. 84, 436-52.

McNally, R.J., Metzger, L.J., Lasko, N.B., Clancy, S.A., Pitman, R.K., 1998. Directed forgetting of trauma cues in adult survivors of childhood sexual abuse with and without post-traumatic stress disorder. J. Abnorm. Psychol. 107, 596-601.

Morin, J.-P., Guzmán-Ramos, K., Bermudez-Rattoni, F., 2015. New Insights on Retrieval-Induced and Ongoing Memory Consolidation: Lessons from Arc. Neural Plast. 2015, 184083.

Nader, K., Schafe, G.E., Le Doux, J.E., 2000. Fear memories require protein synthesis in the amygdala for reconsolidation after retrieval. Nature $406,722-6$.

O’Brien, W.T., Harper, A.D., Jové, F., Woodgett, J.R., Maretto, S., Piccolo, S., 
Klein, P.S., 2004. Glycogen synthase kinase-3beta haploinsufficiency mimics the behavioural and molecular effects of lithium. J. Neurosci. 24, 6791-8.

Olff, M., Langeland, W., Gersons, B.P.R., 2005. Effects of appraisal and coping on the neuroendocrine response to extreme stress. Neurosci. Biobehav. Rev. 29, 457-67.

Pavlov, I.P., 1927. Conditioned reflexes: an investigation of the physiological activity of the cerebral cortex / Translated and edited by G V. Anrep. Oxford University Press, London, 1927. 442-450.

Peineau, S., Bradley, C., Taghibiglou, C., Doherty, A., Bortolotto, Z.A., Wang, Y.T., Collingridge, G.L., 2008. The role of GSK-3 in synaptic plasticity. Br. J. Pharmacol. 153 Suppl, S428-37.

Peineau, S., Nicolas, C.S., Bortolotto, Z.A., Bhat, R. V, Ryves, W.J., Harwood, A.J., Dournaud, P., Fitzjohn, S.M., Collingridge, G.L., 2009. A systematic investigation of the protein kinases involved in NMDA receptordependent LTD: evidence for a role of GSK-3 but not other serine/threonine kinases. Mol. Brain 2, 22.

Peineau, S., Taghibiglou, C., Bradley, C., Wong, T.P., Liu, L., Lu, J., Lo, E., Wu, D., Saule, E., Bouschet, T., Matthews, P., Isaac, J.T.R., Bortolotto, Z.A., Wang, Y.T., Collingridge, G.L., 2007. LTP inhibits LTD in the hippocampus via regulation of GSK3 $\beta$. Neuron 53, 703-17.

Polter, A., Beurel, E., Yang, S., Garner, R., Song, L., Miller, C.A., Sweatt, J.D., McMahon, L., Bartolucci, A.A., Li, X., Jope, R.S., 2010a. Deficiency in the Inhibitory Serine-Phosphorylation of Glycogen Synthase Kinase-3 Increases Sensitivity to Mood Disturbances. Neuropsychopharmacology.

Porsolt, R.D., Bertin, A., Jalfre, M., 1977. Behavioural despair in mice: a primary screening test for antidepressants. Arch. Int. Pharmacodyn. therapie 229, 327-36.

Pryce, C.R., Azzinnari, D., Spinelli, S., Seifritz, E., Tegethoff, M., Meinlschmidt, G., 2011. Helplessness: a systematic translational review of theory and evidence for its relevance to understanding and treating depression. Pharmacol. Ther. 132, 242-67.

Rangel, A., Gonzalez, L.E., Villarroel, V., Hernandez, L., 2003. Anxiolysis 
followed by anxiogenesis relates to coping and corticosterone after medial prefrontal cortical damage in rats. Brain Res. 992, 96-103.

Reist, C., Duffy, J.G., Fujimoto, K., Cahill, L., 2001. beta-Adrenergic blockade and emotional memory in PTSD. Int. J. Neuropsychopharmacol. 4, 37783.

Richardson, M.P., Strange, B.A., Dolan, R.J., 2004. Encoding of emotional memories depends on amygdala and hippocampus and their interactions. Nat. Neurosci. 7, 278-85.

Rohleder, N., Joksimovic, L., Wolf, J.M., Kirschbaum, C., 2004. Hypocortisolism and increased glucocorticoid sensitivity of proInflammatory cytokine production in Bosnian war refugees with posttraumatic stress disorder. Biol. Psychiatry 55, 745-51.

Sapolsky, R.M., Romero, L.M., Munck, A.U., 2000. How do glucocorticoids influence stress responses? Integrating permissive, suppressive, stimulatory, and preparative actions. Endocr. Rev. 21, 55-89.

Sheehan, T.P., Neve, R.L., Duman, R.S., Russell, D.S., 2003. Antidepressant effect of the calcium-activated tyrosine kinase Pyk2 in the lateral septum. Biol. Psychiatry 54, 540-51.

Shi, X., Miller, J. S., Harper, L. J., Poole, R. L., Gould, T. J., Unterwald, E. M., 2014. Reactivation of cocaine reward memory engages the Akt/GSK3/mTOR signaling pathway and can be disrupted by GSK3 inhibition. Psychopharmacology, 231(16), 3109-18.

Siegmund, A., Wotjak, C.T., 2006. Toward an animal model of post-traumatic stress disorder. Ann. N. Y. Acad. Sci. 1071, 324-34.

Stambolic, V., Ruel, L., Woodgett, J.R., 1996. Lithium inhibits glycogen synthase kinase- 3 activity and mimics wingless signalling in intact cells. Curr. Biol. 6, 1664-8.

Steinvorth, S., Wang, C., Ulbert, I., Schomer, D., Halgren, E., 2010. Human entorhinal gamma and theta oscillations selective for remote autobiographical memory. Hippocampus 20, 166-73.

Steru, L., Chermat, R., Thierry, B., Simon, P., 1985. The tail suspension test: a new method for screening antidepressants in mice. Psychopharmacology (Berl). 85, 367-70. 
Stone, E.A., Lin, Y., Quartermain, D., 2008. Evaluation of the repeated openspace swim model of depression in the mouse. Pharmacol. Biochem. Behav. 91, 190-5.

Strekalova, T. V, 1995. The characteristics of the defensive behaviour of rats in accordance with their resistance to emotional stress. Zh. Vyssh. Nerv. Deiat. Im. I P Pavlova 45, 420-2.

Strekalova, T., Couch, Y., Kholod, N., Boyks, M., Malin, D., Leprince, P., Steinbusch, H.M., 2011. Update in the methodology of the chronic stress paradigm: internal control matters. Behav. Brain Funct. 7, 9.

Takahashi, M., Tomizawa, K., Kato, R., Sato, K., Uchida, T., Fujita, S.C., Imahori, K., 1994. Localization and developmental changes of tau protein kinase 1/glycogen synthase kinase-3 beta in rat brain. J. Neurochem. 63, 245-55.

van Stegeren, A.H., 2008. The role of the noradrenergic system in emotional memory. Acta Psychol. (Amst). 127, 532-41.

Varela, C., Weiss, S., Meyer, R., Halassa, M., Biedenkapp, J., Wilson, M.A., Goosens, K.A., Bendor, D., 2016. Tracking the Time-Dependent Role of the Hippocampus in Memory Recall Using DREADDs. PLoS One 11, e0154374.

Warden, M.R., Selimbeyoglu, A., Mirzabekov, J.J., Lo, M., Thompson, K.R., Kim, S.-Y., Adhikari, A., Tye, K.M., Frank, L.M., Deisseroth, K., 2012. A prefrontal cortex-brainstem neuronal projection that controls response to behavioural challenge. Nature 492, 428-32.

Weber, H., Kittel-Schneider, S., Gessner, A., Domschke, K., Neuner, M., Jacob, C.P., Buttenschon, H.N., Boreatti-Hümmer, A., Volkert, J., Herterich, S., Baune, B.T., Gross-Lesch, S., Kopf, J., Kreiker, S., Nguyen, T.T., Weissflog, L., Arolt, V., Mors, O., Deckert, J., Lesch, K.-P., Reif, A., 2011. Cross-disorder analysis of bipolar risk genes: further evidence of DGKH as a risk gene for bipolar disorder, but also unipolar depression and adult ADHD. Neuropsychopharmacology 36, 2076-85.

Wingenfeld, K., Wolf, O.T., 2014. Stress, memory, and the hippocampus. Hippocampus Clin. Neurosci. 34, 109-20.

Wolf, O.T., 2009. Stress and memory in humans: twelve years of progress? Brain Res. 1293, 142-54. 
Wood, N.E., Rosasco, M.L., Suris, A.M., Spring, J.D., Marin, M.-F., Lasko, N.B., Goetz, J.M., Fischer, A.M., Orr, S.P., Pitman, R.K., 2015. Pharmacological blockade of memory reconsolidation in post-traumatic stress disorder: three negative psychophysiological studies. Psychiatry Res. 225, 31-9.

Yehuda, R., 2009. Status of glucocorticoid alterations in post-traumatic stress disorder. Ann. N. Y. Acad. Sci. 1179, 56-69.

Yehuda, R., Harvey, P.D., Buchsbaum, M., Tischler, L., Schmeidler, J., 2007a. Enhanced effects of cortisol administration on episodic and working memory in aging veterans with PTSD. Neuropsychopharmacology 32, 2581-91.

Yehuda, R., Joëls, M., Morris, R.G.M., 2010. The memory paradox. Nat. Rev. Neurosci. 11, 837-9.

Yehuda, R., LeDoux, J., 2007. Response variation following trauma: a translational neuroscience approach to understanding PTSD. Neuron $56,19-32$. 


\title{
Chapter 2. Hippocampal gene expression of deiodinases 2 and 3 and effects of 3,5-diiodo-L-thyronine T2 in mouse depression paradigms
}

\begin{abstract}
Nataliia Markova ${ }^{1}$, Anton Chernopiatko ${ }^{2}$, Careen A. Schroeter ${ }^{3}$, Dmitry Malin $^{4,5}$, Aslan Kubatiev ${ }^{5}$, Sergey Bachurin ${ }^{1}$, João Costa-Nunes ${ }^{6,7}$, Harry M. W. Steinbusch ${ }^{6}$, and Tatyana Strekalova ${ }^{3,6,7}$

${ }^{1}$ Institute of Physiologically Active Compounds, Russian Academy of Sciences, Severnii proezd 1, Chernogolovka, Moscow Region 142432, Russia; ${ }^{2}$ Timantti AB, Sundbyberg 104 , 17407 Stockholm, Sweden; ${ }^{3}$ Department of Preventive Medicine, Maastricht Medical Center in Annadal, Becanusstraat 17 A0, 6216 BX Maastricht, The Netherlands; ${ }^{4}$ Carbone Cancer Center, University ofWisconsin, WIMR 3016, 1111 Highland Avenue, Madison, WI 53705, USA; ${ }^{5}$ Institute of General Pathology and Pathophysiology, Russian Academy of Medical Sciences, Baltiyskaia 8, Moscow 125315, Russia; ${ }^{6}$ Department of Neuroscience, School for Mental Health and Neuroscience, Maastricht University, Universiteitssingel 40, NL 6229 ER Maastricht, The Netherlands; ${ }^{7}$ Institute for Hygiene and Tropical Medicine, New University of Lisbon, Rua da Junqueira 96, 1349-008 Lisbon, Portugal.
\end{abstract}

Correspondence should be addressed to Tatyana Strekalova:

strekalova@maastrichtuniversity.nl

Markova, N., Chernopiatko, A., Schroeter, C.A., Malin, D., Kubatiev, A., Bachurin, S., Costa-Nunes, J., Steinbusch, H.M.W., Strekalova, T., 2013. Hippocampal gene expression of deiodinases 2 and 3 and effects of 3,5diiodo-L-thyronine T2 in mouse depression paradigms. Biomed Res. Int. 2013, 565218. 


\section{Abstract}

Central thyroid hormone signaling is important in brain function/dysfunction, including affective disorders and depression. In contrast to 3,3',5-triiodo-L-thyronine (T3), the role of 3,5-diiodo-L-thyronine (T2), which until recently was considered an inactive metabolite of T3, has not been studied in these pathologies. However, both T3 and T2 stimulate mitochondrial respiration, a factor counteracting the pathogenesis of depressive disorder, but the cellular origins in the CNS, mechanisms, and kinetics of the cellular action for these two hormones are distinct and independent of each other. Here, Illumina and RT PCR assays showed that hippocampal gene expression of deiodinases 2 and 3, enzymes involved in thyroid hormone regulation, is increased in resilience to stress-induced depressive syndrome and after antidepressant treatment in mice that might suggest elevated T2 and T3 turnover in these phenotypes. In a separate experiment, bolus administration of T2 at the doses 750 and $1500 \mathrm{mcg} / \mathrm{kg}$ but not $250 \mathrm{mcg} / \mathrm{kg}$ in naive mice reduced immobility in a two-day tail suspension test in various settings without changing locomotion or anxiety. This demonstrates an antidepressant-like effect of $\mathrm{T} 2$ that could be exploited clinically. In a wider context, the current study suggests important central functions of T2, whose biological role only lately is becoming to be elucidated.

\section{Introduction}

Despite advances in the pharmacotherapy of depression, many patients fail to respond to standard antidepressants. This requires new treatment and augmentation approaches to be developed. Further elaboration of a potential of the brain thyroid system to be targeted to elicit an antidepressant action can be one of the most promising strategies. Central thyroid hormone synthesis was demonstrated in the dentate gyrus of the hippocampus, the septum, amygdala, and the olfactory bulb [1-5]. The action of thyroid hormones in the CNS is considered to be independent of peripheral thyroid hormones [5-7]. At very low doses, brain thyroid hormones induce profound effects on the CNS. For example, they enhance hippocampal neurogenesis [8] and the secretion of neurotrophins, including 
BDNF, either directly or via monoamine receptors, and they activate PI3 KAkt signaling through integrin receptors [9-11]. Deficiency of brain thyroid hormone production contributes to reduced central serotonin activity and development of depression [6, 12].

A number of studies suggest that both 3,3,5-triiodo-L-thyronine (T3) and 3,5, $3^{\prime}, 5^{\prime}$-tetraiodo-L-thyronine (T4) thyroid hormones could be promising adjunct therapy in patients refractory to tricyclics and selective serotonin reuptake inhibitors (SSRI) $[13,14]$. Hitherto, it was not clear whether 3,5diiodo-L-thyronine (T2), which has been recently identified as functionally active metabolite of T3 in periphery in vivo and in vitro systems, might have similar effects to T3 that might be exploited in a clinic. New evidence does suggest that T2may mediate the effects of antidepressant therapy in the brain. A two-week intraperitoneal administration of the tricyclic antidepressant desipramine in the rat induces the expression of $\mathrm{T} 2$ in the amygdala [2]. This was paralleled by increased concentrations of T3 in nuclei but in contrast to $\mathrm{T} 2$ expression not in the mitochondria. These desipramineinduced changes in T2 were accompanied by an increase in the concentrations of succinate dehydrogenase in the mitochondria, suggesting their elevated functional activity [2]. Other experiments of the same group [1] have shown the presence of metabolites of $\mathrm{T} 2$ in several brain areas including the hippocampal formation and septum that points to the occurrence of $\mathrm{T} 2$ itself in the limbic system. Extensive study of Eravci and coworkers [15] has shown significant changes in the hippocampal formation and amygdala in the key enzymes regulating the levels of thyroid hormones, deiodinases 2 (DIO2) and 3 (DIO3) of the rats which were dosed with antidepressant and antipsychotic agents or subjected to a $24 \mathrm{~h}$ sleep deprivation, a commonly used non-pharmacological antidepressant treatment. Thus changes in the levels of thyroid hormones by their synthesis or degradation in the limbic system generally and in the hippocampus in particular are implicated in depressive traits and antidepressant response.

T2 was recently found to increase resting metabolic rate and prevent dietinduced insulin resistance through the stimulation of the mitochondrial respiratory chain; the latter mechanism is in itself an emerging target of new 
antidepressant therapies [16-21]. These and other studies have revealed intriguing cellular effects of T2 that seem to be distinct from that of T3. Unlike T3, T2 enhances mitochondrial respiration by a nuclear-independent mechanism and neither does act via thyroid hormone receptor beta, nor via AMP-activated protein kinase. T2 was demonstrated to activate reactions involved in substrate oxidation, affecting both cytochrome $\mathrm{c}$ reducers and cytochrome c oxidizers [20,21], and besides it effects on respiratory chain, it was suggested to increase the downstream mechanisms that are involved in mitochondrial biogenesis [17]; these and other effects of T2onmitochondrial pathways were shown to be distinct from the effects of T3 [22]. In contrast to the lasting I effects of T3 on metabolic rate and mitochondrial respiration, with onset delayed for $48 \mathrm{~h}$, the action of T2 appears within the first few hours and does not persist for longer than $48 \mathrm{~h}$ and is not sensitive to actinomycin $D[5,20,22]$. T2 was found to evoke selective thyromimetic activity in vitro and in vivo that is divergent from that of T3 [23]. This and other mounting evidence, suggest that the effects of $\mathrm{T} 2$ do not merely mimic those of T3 but, instead, involve distinct mechanisms that seem likely to be related to stimulatory action on mitochondria.

Intriguingly, there is growing evidence to suggest that antidepressants can stimulate the mitochondrial respiratory chain directly and indirectly and suggest that these effects are implicated in the stress response and the pathogenesis of a depressive-like state. For instance, thiazolidinediones, which act as potent sensitizers of the neuronal insulin receptor, enhance brain glucose utilization though increased neuronal mitochondrial biogenesis [24], decrease neuronal damage [25], induce an antidepressant-like effect in the tail suspension and forced swim tests in mice [26], and show clinical efficacy in patients with major depression $[27,28]$. Our recent studies on mice have indicated that dicholine succinate, a molecule that stimulates insulin-dependent $\mathrm{H} 2 \mathrm{O} 2$ production of the mitochondrial respiratory chain, decreased signs of stressed-induced anhedonia in a sucrose test, immobility in the forced swim model, and hippocampal gene expression [29].

So far, limited efforts have been made to address potential central functions of T2 using animal models of depression. The hippocampus, as part of the 
limbic system, was chosen as a focus of the present study based on the striking differences between resilient versus susceptible to stress-induced depressive-like state mice in the mitochondrial gene expression of this structure [30, 31]. Using the above-mentioned paradigm of stress-induced anhedonia in C57BL6J mice, we addressed whether hippocampal expression of the key enzymes regulating the levels of thyroid hormones, DIO2 and DIO3, is altered in the hippocampus of animals with depressive-like features. Separately, we also assessed the effects of a bolus administration of 3,5diiodo-L-thyronine (T2) in mice in the tail suspension test, a common test of depressive-like behaviour, and in supplementary paradigms for anxiety and locomotion, such as dark-light and novel cage tests. The efficacy of the treatment with T2 was evaluated on two laboratory strains, C57BL6J and CD1 mice. A selection of a two-day tail suspension paradigm as a test for a depressive-like behaviour [32] and of single dosing were based on previously reported studies, in which $\mathrm{T} 2$ induced marked effects in rats within the first hours after administration [16, 33] and chronic administration of T2 evoked thyromimetic action in rats [23] that we wished to avoid in our study. Therefore, T2 was applied to mice intragastrically as a bolus injection $30 \mathrm{~min}$ prior to or $2 \mathrm{~h}$ after the first test session.

\section{Materials and Methods}

\section{Animals and Housing}

Male C57BL6J and CD1 mice were 3-month old. Two-five-month-old Wistar rats of the same age were used for predator stress. Mice were obtained from Charles River (Janvier, l'Arbresle Cedex, France) and rats from the Medical Faculty of New University of Lisbon, Lisbon, Portugal. 14 days before the behavioural experiments mice were single housed under a reverse $12 \mathrm{~h}$ : $12 \mathrm{~h}$ light-dark cycle (lights on: 21:00 h) in standard laboratory conditions (22 $\pm 1^{\circ} \mathrm{C}, 55 \%$ humidity, food, and water ad libitum). All experiments were carried out in accordance with the European Committees Council Directives and had been approved by the state governmental bodies of animal care and welfare. 


\section{Chronic Stress Experiment}

\section{Experimental Conditions and Study Outline.}

Parameters of social behaviour were determined one week before the chronic stress procedure in a social interaction test as described elsewhere $[30,34,35]$. Body weight and baseline preference for a $1 \%$ sucrose solution (see Sucrose Test) were recorded (Figure 1(a)). The experimental and control groups were balanced upon these parameters. Together, 40 mice were assigned to a stress group and 24 controls constituted a nonstressed control group. Among animals from a stress group, twenty mice received no treatment and twenty were treated with imipramine. Control mice were neither treated $(n=14)$ nor treated with imipramine $(n=10)$. In control and stress groups, imipramine $(7 \mathrm{mg} / \mathrm{kg} / \mathrm{day})$ was administrated via drinking water starting 7 days prior to the onset of stress and lasting the entire duration of the stress procedure. The current antidepressant treatment was applied as described elsewhere [29].

Additionally to baseline measurements, a sucrose consumption test was performed on the 10th day of the chronic stress procedure. On the next day, mice were tested in the tail suspension test and scored for a coat disintegration, and their body weight was evaluated. Five days after the termination of the stress procedure, mice were sacrificed for gene expression analysis (Figure $1(a)$ ).

\section{(1) Chronic Stress Procedure.}

This study uses a recently validated variant of a 10-day stress protocol [29, $34,35]$ comprising nighttime rat exposure and day time application of two stressors: a social defeat and restraint stress. Between the hours of 10:00 and 17:00, social defeat for 30 minutes and restraint stress were employed for 2 hours with an intersession interval of at least 4 hours.

Rat Exposure While in a Small Container. Mice were introduced to transparent glass cylindrical containers $(15 \mathrm{~cm} \times 0.8 \mathrm{~cm})$ and placed into the rat cage ( $15 \mathrm{~h}$ exposures were performed between 18.00 and 09.00). 
Social Defeat Stress. Social defeat procedures took place during the dark phase; to enable a visual control over the resident-intruder confrontation, the test was carried out under red light. In a preliminary test, aggressive individuals of the CD1mouse strain that were able to attack the counter partners in less than $60 \mathrm{~s}$ without injuring them were selected for this procedure; these animals were introduced in the home cages of mice from the stress group during social defeat sessions. Social interaction was set up in the home cage of stressed animals as it enhances the impact of the stress procedure in a lasting manner. In a variant procedure, a defeated animal is left in chronic contact with the olfactory cues of the aggressive intruder, such that exposure to a psychological stressor is chronic, although the actual agonistic experience is intermittent. Average duration of each session was $30 \mathrm{~min}$ in accordance with commonly used protocols. During social defeat stress, test mice typically showed flight response, submissive posture, and vocalization. Pairs of animals were carefully observed in order to exclude any physical harm. In rare cases of its incidence, aggressive individuals were immediately removed from the cage of resident mice.

Restraint Stress. Animals were placed inside plastic tubes (internal diameter is app. $26 \mathrm{~mm}$ ) during the dark phase of the light cycle. Small standard pieces of tissue paper were inserted in the tubes to restrict animals' activity.

\section{(2) Assessment of Stress Effects}

Sucrose Test. Animals were given $8 \mathrm{~h}$ of free choice between two bottles of either $1 \%$ sucrose or normal drinking water. At the beginning and end of the period, the bottles were weighed and consumption was calculated. The beginning of the test started with the onset of the dark (active) phase of animals' cycle. To prevent the possible effects of side preference in drinking behaviour, the position of the bottles in the cage was switched at $4 \mathrm{~h}$, halfway through testing. No previous food or water deprivation was applied before the test. Other conditions of the test were applied as described elsewhere [24]. The $1 \%$ sucrose solution is used in tests performed during baseline and chronic stress application. Percentage preference for sucrose is calculated using the following formula: 
sucrose preference

$$
=\left[\frac{\mathrm{V}(\text { Sucrose solution })}{\mathrm{V}(\text { Sucrose solution })+\mathrm{V}(\text { Water })}\right] \times 100 \% \text {. }
$$

A decrease in sucrose preference to a level below $65 \%$ measured at the 10th day of continuous stress application was taken as a criterion for anhedonia. This criterion was based on the fact that none of the control animals exhibited $<65 \%$ preference for sucrose at that time point of the study and, accordingly, mice exhibiting a sucrose preference of $<65 \%$ were defined as susceptible to stress-induced anhedonia. Mice that had undergone stress but maintained a sucrose preference of $>65 \%$ were defined as resilient to this state. In addition, our previous results indicated that mice matching this criterion showed a depressive-like syndrome [35]. This procedure induces anhedonia in a considerably shorter time than previously validated models by increasing the daytime stress load.

Tail Suspension Test. The protocol used in this study was adapted from a previously proposed procedure [36]. Mice were subjected to the modified tail suspension by being hung by their tails with adhesive tape to a rod $50 \mathrm{~cm}$ above the floor for $2 \mathrm{~min}$. Two animals were tested simultaneously in a dark room where only the area of the modified tail suspension construction was illuminated by a spotlight from the ceiling; the lighting intensity on the height of the mouse position was 25 Lux. The trials were recorded by a video camera positioned directly in front of the mice, while the experimenter observed the session from a distance in a dark area of the experimental room. This procedure was carried out twice with a $24 \mathrm{~h}$ interval between tests, similarly to previously reported protocols [32]. The latency of the first episode of immobility and the total duration of this behaviour were scored manually according to the protocol that was previously validated with automated scoring using CleverSys software [36]. In accordance with the commonly accepted criteria of immobility, the immobility behaviour was defined as the absence of any movements of the animals' head and body. The latency of immobility was determined as the time between the onset of the test and the first bout of immobility. 
Coat State Scoring. Ac oat state was scored in a blinded fashion to assess a stress-related disintegration of fur. Scores from 1 (very poor) to 5 (excellent) were assigned to each animal by two independent experimenters; the average note was taken. This parameter was earlier shown to correlate with a development of stress-induced anhedonia as it reflects selfgrooming behaviour that is dependent on hedonic sensitivity [36].

Brain Dissection, RNA Isolation, and Illumina Microarray Gene Expression Profiling. Mice were sacrificed by cervical dislocation. RNA extraction was performed from microdissected snap-frozen hippocampus using RNeasy RNA extraction kit with DNasel treatment, as previously described (Qiagen, Hilden, Germany; [34]).

Gene expression profiling was performed using Illumina technology (Integragen, Evry, France, and Northwestern Chicago University, USA) with the hippocampus of mice from nonstressed control (drug-naive or treated with imipramine), stressed resilient, and anhedonic groups and from stressed imipramine-treated mice (five animals per each group were analyzed). Total RNA samples were hybridized to Illumina BeadChips (MouseRef-8 v2 Expression BeadChip; Illumina, Inc. San Diego, CA, USA) which were prepared using the Illumina TotalPrep RNA Amplification kit (Applied Biosystems/Ambion, Carlsbad, CA, USA); the samples were assigned to the chips in random order with the constraint that no two samples from the same group were assigned to the same chip to avoid confounding of experimental groups with the chips. Microarray data were analyzed using standard analysis procedures, which included assessment of the overall quality of array data and statistical evaluation of differentially expressed genes (IntegraGen, Evry, France). Once the quality of array data was confirmed, the Gene Chip Operating System (Illumina, Inc., San Diego, CA, USA) was used to calculate signal intensities, detection calls, and their associated $p$ values for each transcript on the array. Gene expression was normalized to the expression of the housekeeping gene, beta-actin, due to its stable expression, and calculated as percent mean of the respective control group (pharmacologically naive or treated with imipramine). 
Differences in gene expression between groups were evaluated using unpaired two-tailed t-test.

Real-Time PCR (RT-PCR). $1 \mathrm{~g}$ total RNA was converted into CDNA as described elsewhere [34]. RT-PCR was run using SYBR green based technology (Primer Design Ltd., Southampton, UK). Standard curves were generated using previously generated samples to enable normalization to the housekeeping gene glyceraldehyde-3-phosphate dehydrogenase (GAPDH; forward primer ACCCCTTCATTGACCTCAACTACATG;

reverse primer CCTTCTCCATGGTGGTGAAGAC) using the Pfaffl method as described elsewhere [37]. Expression of DIO2 was assessed using a forward primer GATGCTCCCAATTCCAGTGT and reversed primer TGAACCAAAGTTGACCACCA; expression ofDIO3 was assessed using a forward primer CCGCATATGGTGCCTATTTT and reversed primer GCCCACCAATTCAGTCACTT. All samples were run in duplicate. Cycling was performed at $95^{\circ} \mathrm{C}$ for $5 \mathrm{~min}$ followed by a 45 cycle amplification at $95^{\circ} \mathrm{C}$ for $10 \mathrm{~s}$, then at the annealing temperature for $15 \mathrm{~s}$ and at the temperature $72^{\circ} \mathrm{C}$ for $20 \mathrm{~s}$. Results of the qPCR measurements were expressed as $\mathrm{Ct}$ values, where $\mathrm{Ct}$ is defined as the threshold cycle of PCR at which amplified product was $0.05 \%$ of normalized maximal signal. We used the comparative $\mathrm{Ct}$ method and computed the difference between the expression of the gene of interest and GAPDH expression in each cDNA sample (2- $\Delta \Delta \mathrm{Ct}$ method). Results are expressed as relative-fold change compared to control animals.

Tail Suspension Experiment. On the basis of gene expression data, the further aim of our study is to examine the effects of bolus T2 administration on the immobilization behaviour in the tail suspension test in C57BL6J and CD1 mice. Supplementary tests for anxiety-like behaviour and locomotion were carried out on the same mouse strains in order to rule out potential confounds with the evaluation of behavioural despair after the treatment with T2.

First, we investigated the effects of a bolus oral gavage of T2 (see drug administration below) to C57BL6J mice at the dose of $250 \mathrm{mcg} / \mathrm{kg}$ either 30 min before $(n=7)$ or $2 \mathrm{~h}$ after $(n=8)$ the first tail suspension session of the test (Figures 1 (b) and $1(\mathrm{c})$ ), as described previously [26, 32, 36]; control 
groups constituted 8 mice in each experiment. Second, T2 was used at the dose of $750 \mathrm{mcg} / \mathrm{kg}$ prior to the tail suspension session $(\mathrm{n}=8)$ or $2 \mathrm{~h}$ thereafter $(n=8)$; control groups constituted 8 mice. Finally, a dose of 1500 $\mathrm{mcg} / \mathrm{kg}$ was applied 30 min prior to the first tail suspension session in CD1 mice $(n=10)$; control group was formed by 10 mice. The same vehicle was injected into controls. A selection of doses of $\mathrm{T} 2$ and the timing of dosing were based on previously reported studies, in which it induced marked effects in rats within the first hours after administration [16, 33].

In an additional study, we addressed the question of whether the administration of $750 \mathrm{mcg} / \mathrm{kg}$ of T2 in C57BI6J and $750 \mathrm{mcg} / \mathrm{kg}$ of T2 or 1500 $\mathrm{mcg} / \mathrm{kg}$ of this drug in CD1 mice interferes with anxiety and locomotion in a dark-light and novel cage tasks in previously calibrated protocols [36, 38, 39]. Dark-light and novel cage tasks were applied with a $5 \mathrm{~min}$ interval $30 \mathrm{~min}$ after a dosing (Figure 1(d)). All groups constituted 9 mice.

Dark-light Box. The dark-light box (TechnoSmArt, Rome, Italy) consisted of two plexiglass compartments, one black/dark $(15 \mathrm{~cm} \times 20 \mathrm{~cm} \times 25 \mathrm{~cm})$ and one lit $(30 \mathrm{~cm} \times 20 \mathrm{~cm} \times 25 \mathrm{~cm})$, connected by a tunnel. Anxiety-like behaviour was assessed by earlier validated measures. Mice were placed into the dark compartment, from where they could visit the lit box (light intensity 25 Lux). The latency of the first exit to the light compartment, the total duration of time spent in the lit box, and the number of visits to this anxiety-related compartment were scored by visual observation over $5 \mathrm{~min}$.

Novel Cage Test. The novel cage test was performed to assess vertical activity [35, 36, 39]. Mice were introduced into a standard plastic cage; the size of their home cage filled with small amounts of fresh sawdust. The number of exploratory rearings was counted under red light during a 5-min period. The testing was carried out in a dark quiet room in morning hours. Behaviour was videotaped and analyzed by trained observers blind to the animals' treatment.

Drug Administration. Imipramine (Sigma-Aldrich, St. Louis, MO, US) was dissolved in tap water; the solution was freshly prepared every 2-3 days and placed in lightproof drinking bottles. The calculation of the concentration of 
imipramine in drinking water was based on the previously evaluated mean volume of daily water consumption in C57BL6 mice that was about $3 \mathrm{~mL}$ and on the dosage of treatment. Dosage for imipramine was set at $7 \mathrm{mg} / \mathrm{kg} / \mathrm{day}$ as previous studies showed that chronic administration of imipramine at 15 $\mathrm{mg} / \mathrm{kg} /$ day with drinking water, but not $7 \mathrm{mg} / \mathrm{kg} /$ day, significantly affects sucrose intake and locomotor behaviour in naive C57BL6N mice [39]. Imipramine was delivered with drinking water starting 1 week before the onset of stress and then throughout the entire duration of the chronic stress procedure.

3,5-Diiodo-L-thyronine T2 (Sigma-Aldrich, Cambridge, MA, USA) was first dissolved in $100 \%$ DMSO (Sigma-Aldrich, St. Louis, MO, US), the solution was diluted, so the final concentration of DMSO that was $0.2 \%$ and administrated to mice via a single intragastrical administration. The same treatment method was applied to administer a vehicle $0.2 \%$ DMSO solution. A separate control group of mice was injected with saline; no effects of $0.2 \%$ DMSO solution were found in protocols applied here of the tail suspension tests (Strekalova and Chernopiatko, unpublished results). The volume of T2 and vehicle injections was $0.1 \mathrm{~mL} / 10 \mathrm{~g}$ of body weight.

Statistics. Data were analysed with GraphPad Prism version 5.00 for Windows, San Diego, CA. One- and two-way ANOVA were used followed by a Mann-Whitney t-tests post hoc comparisons. Two-tailed unpaired t-tests were applied for two-group, two-tailed comparisons of independent data sets with normal distribution. The level of confidence was set at $95 \%$ ( $p<$ 0.05 ) and data are shown as mean \pm SEM.

\section{Results}

Assessment of Anhedonia Induction. In the model employed, the induction of a depressive-like state by stress exposure was defined by the occurrence of behavioural manifestations of anhedonia, as determined by a decrease in preference for a $1 \%$ sucrose solution to below 65\% [35]. Previous studies have shown that the occurrence of stress-induced anhedonia is associated with a number of behavioural, physiological and molecular features of a depressive state [29-31, 34-36]. 
When assigned to experimental groups, but before being subjected to the chronic stress procedure, groups did not differ in terms of sucrose preference, intake of sucrose solution and water, or body weight $(p>0.05$; one-way ANOVA; data not shown). Two-way ANOVA revealed a significant effect of stress on sucrose preference $\left(F_{1.59}=7.671 ; p=0.0075\right)$. A post hoc Mann-Whitney test showed a significant difference in sucrose preference between control and the non treated stressed group $(U=47.00 ; p=0.0012)$, but when treated with imipramine there was no difference from control and stressed groups in terms of sucrose preference $(U=135.0 ; p=0.8748)$. This suggests that imipramine prevents the reduction in sucrose preference for the stressed cohort (Figure 2(a)). 60\% of non treated stressed mice (12 out of 20 ) and $25 \%$ of imipramine-treated stressed animals (5 out of 20) showed a sucrose preference below $65 \%$ and were defined as anhedonic (susceptible).

Effects of Treatment on Immobility Behaviour. Previous studies have shown that stress-induced anhedonia, measured by the sucrose preference test, is frequently associated with increased "behavioural despair" in the Porsolt forced swim and tail suspension paradigms [40]. Onset of immobility (behavioural despair) in the latter model is defined as the point at which the animal stops struggling for longer than $1 \mathrm{~s}$. Latency to immobility was significantly altered by stress as revealed by two-way ANOVA $\left(F_{1.49}=17.70 ; p\right.$ $=0.0001)$. Post hoc Mann-Whitney tests showed a strong trend towards a significant reduction in latency to immobility for stressed non treated animals compared with control mice $(U=60.50 ; p=0.0889)$; there was no significant difference between stressed and control mice that received imipramine $(U=76.50 ; p=0.2213$; Figure $2(b))$. Time spent immobile showed a significant main effect of stress $\left(F_{1.49}=24.79 ; p<0.0001\right)$; stressed mice were immobile for significant longer than controls (Figure 2(c)). Post hoc analysis showed significant differences between control and stressed non treated groups ( $u=27.00 ; p=0.0012$ ) but not between control and stressed imipramine-treated group ( $u=67.50 ; p=0.1056$ ) suggesting that applied treatment prevents the behavioural despair induced by the chronic stress paradigm. 
Evaluation of Coat Scores. Before the onset of the chronic stress procedure, all mice had good coat quality, with no significant difference between the groups (data not shown). After completion of the chronic stress procedure, a two-way ANOVA comparison showed significant main effects of both stress $\left(F_{1.60}=125.8 ; p<0.0001\right) ;$ and treatment $\left(F_{1.60}=8.416 ; p=0.0052 ;\right.$ Figure 2(d)). All stressed mice showed significantly lower scores of coat state than control-treated animals $(p<0.001)$. However, imipramine-treated animals exhibited higher scores of coat state compared with the non treated group $(U=80.00 ; p=0.0009)$.

Changes in Body Weight. At the end of the chronic stress paradigm, there was a significant difference in body weight between control and stressed animals (two-way ANOVA; $F_{1.60}=65.39 ; p<0.0001$ ), and stressed drug-naive group showed a significant decrease in body weight compared with controls $(U=21.5, p<0.0001$ for stressed non treated animals), while imipraminetreated mice did not show such a change $(U=100.5 ; p=0.1718$; Figure 2(e)). Stressed mice treated with an antidepressant were significantly heavier than those that were not treated pharmacologically $(U=111.5 ; p=0.0172$; Figure 2(e)).

Expression of Genes Encoding Deiodinases 2 and 3 in the Hippocampus of Stressed Mice. There was a trend towards elevatedDIO2 expression for resilient versus susceptible mice as shown by Illumina microarray analysis ( $p$ $=0.10 ; \mathrm{t}=1.362, \mathrm{df}=6$; unpaired t-test; Figure 3(a)), and RT-PCR revealed an increase in DIO2 expression in resilient versus susceptible mice $(U=2.000 ; p$ $=0.0488$; Mann-Whitney test; Figure 3(c)). Both Illumina profiling and RTPCR assays have shown that DIO2 was significantly augmented in the stressed imipramine-treated group in comparison with stressed drug-naive animals $(p=0.04 ; t=1.797 ; d f=16$; $t$-test and $U=3.000 ; p=0.0424$; MannWhitney test, resp.; Figures $3(b)$ and $3(d))$. There was a trend toward elevated DIO3 expression for resilient versus anhedonic mice as shown by Illumina assay $(p=0.11 ; t=0.9755 ; d f=7$; t-test; Figure $3(\mathrm{e}))$ and RT-PCR analysis $(U=3.000, p=0.1250$; Mann-Whitney; Figure $3(\mathrm{~g})$ ). Illumina microarray has demonstrated a non-significant increase in the $\mathrm{DIO} 3$ expression in imipramine-treated animals over this measure in stressed 
drug-naive group $(p=0.137 ; t=1.557 ; d f=18$; t-test; Figure 3(f)). Following this trend, RT-PCR revealed significantly elevated level of DIO3 expression in the stressed imipramine-treated group in comparison with stressed drugnaive animals $(U=3.000 ; p=0.0420$; Mann-Whitney test, resp., Figure 3(h)). Of note, in control nonstressed mice, the expression did not differ for either gene between drug-naïve and imipramine-treated groups (data not shown).

3,5-Diiodo-L-Thyronine T2 Reduces Immobility Time in the Tail Suspension Test. The parameters of immobility in mice dosed with $250 \mathrm{mcg} / \mathrm{kg}$ of T2 in either protocol of the tail suspension test were unaltered (Figures 4(a) and 4(b)). C57BL6J and CD1 mice dosed prior to the first testing with $750 \mathrm{mcg} / \mathrm{kg}$ or $1500 \mathrm{mcg} / \mathrm{kg}$, respectively, had significantly reduced immobility on Day 1 ( $p=0.008, t=3.076, d f=14$ and $p=0.025, t=2.089$, and $d f=18$ ) and Day 2 $(p=0.0205, t=3.327, d f=14$ and $p=0.0096, t=2.572, d f=18$; Figure 4(e) $)$. There was a tendency to longer latency of immobility during these measurements; a significant increase was found onDay 2 of testing of mice treated with $750 \mathrm{mcg} / \mathrm{kg}(\mathrm{p}=0.0169, \mathrm{t}=2.352, \mathrm{df}=14)$. Animals that received $\mathrm{T} 2$ at the dose $750 \mathrm{mcg} / \mathrm{kg}$ of $\mathrm{T} 2$ after the first test session showed a tendency to decreased immobility $(p=0.0734, t=1.536$, and $d f=14)$ and significantly increased latency of immobility $(p=0.006, t=2.840, d f=14)$ on Day 2; experimental groups have displayed no behavioural differences prior to the treatment, on Day 1 (Figure 4(c)).

Dosing with 750 or $1500 \mathrm{mcg} / \mathrm{kg}$ did not alter anxiety-like behaviour of C57BI6J mice (data not shown) and CD1 mice, as shown by unaffected latencies of the first exit to the lit compartment (one-way ANOVA, $F=$ $0.4640, R^{2}=0.0662$, and $p=0.6388$; Figure $5(a)$ ), time spent therein (oneway ANOVA, $F=2.390, R^{2}=0.2688$, and $p=0.1307$; Figure $5(b)$ ), and number of exits (one-way ANOVA, $F=0.07238, R^{2}=0.01101$, and $p=0.9305$; Figure $5(c)$ ). The number of rearings was unaltered (one-way ANOVA, $F=0.1395, R^{2}$ $=0.02101$, and $p=0.8711$; Figure 5(d)).

\section{Discussion}

The present study highlights hippocampal gene expression of $\mathrm{DIO} 2$ and DIO3, enzymes involved in the thyroid hormone regulation, as a molecular 
correlate of antidepressant-like effects in a stress-induced depressive syndrome in mice. The present study also identifies increased hippocampal gene expression ofDIO2 alone in the stress resilient cohort of mice. While these changes in enzyme expression levels may not necessary generate elevated levels of central thyroid hormone, the ability of administrated T2 to reverse parameters of behavioural despair supports the hypothesis for T2 as a contributor to antidepressant activity and suggests that the administration or augmentation of $\mathrm{T} 2$ production may be of value in the clinic.

Stress-induced anhedonia in mice $[34,35,40]$ resulting in decreased intake or preference for palatable solutions (such as a sucrose solution) is interpreted as a decreased ability to experience pleasure, a core feature of human depression [41]. The stressed mice treated with imipramine in this study retained a sucrose preference, which is in agreement with reports from our lab and others, that conventional antidepressant treatments are able to prevent the stress-induced decrease in sucrose preference $[29,42$, 43]. In addition to the effects on sucrose preference, we showed that treatment with imipramine had antidepressant-like effects in the tail suspension test, and coat state was also improved by imipramine. Coat disintegration in rodents is due to reduced motivation for self-cleaning and is used as an important feature of a depressive-like state in preclinical models [44]. Improvements in coat state have accordingly been documented for antidepressant treatments in rodent models of depression [36, 40, 42]. Imipramine diminished stress-induced loss of body weight. It has been previously shown that antidepressant treatment results in a restoration of body weight $[40,42,43]$. Thus, it can easily be argued that imipramine, at the dose employed, has been effective as an antidepressant as a consequence of the improvement observed in all of these outcomes. This is of note because we used a much lower dose than has been used by convention. The use of a lower dose was indicated after we showed that higher doses of imipramine are associated with significant side effects in naive animals [39]. Such side effects might have been expected to confound our experimental outcomes, and thus, it was important to be able to validate this lower dose. 
Illumina microarray data and our RT-PCR assays suggested elevated hippocampal gene expression of DIO2, encoding a key enzyme of the synthesis of T2 and T3 hormones, in stressed mice that received imipramine and in stressed mice that are resilient versus mice susceptible to depressive syndrome. RT-PCR also showed a significant increase in DIO3 expression in stressed imipramine-treated mice, but no other group differences were significant. As DIO2 is an enzyme of thyroid hormone synthesis in the brain, DIO3 inactivates thyroid hormones, and simultaneous activation of both biosynthesis and degradation of neurochemically active factors often accompanies their enhanced turnover. In line with our findings, subchronic antidepressant and antipsychotic treatments in a rat induced more pronounced brain changes of $\mathrm{DIO} 2$ than of $\mathrm{DIO}_{3}$, which have occurred in the same direction [15]. In support of our results, exposure of a rat to $24 \mathrm{~h}$ sleep deprivation, commonly used as non-pharmacological antidepressant treatment, has increased the activities of $\mathrm{DIO} 2$ in the hippocampus and several more brain structures, without affecting DIO3; of note, no such changes were found in the amygdala in these experiments [15]. This study also has shown that desipramine, an antidepressant that is similar to the one used here imipramine, has induced dose-dependent increases of $\mathrm{DIO} 2$ in the cortex, whereas no changes in the $\mathrm{DIO} 3$ were observed [15]. Notably, among deiodinases 1,2 , and 3, DIO2 in particular was found to be the highly sensitive to many different kinds of influences that are believed to be capable of inducing the changes in thyroid hormone concentrations in the CNS and neuronal activity [15]. Other studies showed that mice lacking DIO2 were demonstrated to have deficits in the hippocampus-dependent learning [45], obesity with glucose intolerance [46] that is a well-established indicator $f$ depressive disorder. Since DIO2 gene expression was increased in our study in resilient (nonanhedonic) mice or animals that received imipramine, it can be speculated that elevated synthesis of thyroid hormones in the hippocampus is associated with resilience to a depressive-like syndrome. To the best of our knowledge, no such changes in the $\mathrm{DIO} 2$ have been reported until now in relation to a susceptibility to experimentally induced depressive-like status in animal models of depression. 
Our molecular biology results are indicative of altered central thyroid hormone concentrations, but they are not conclusive, and direct measurements of T2 and T3 in the brain are required. However, the RT-PCR results prompted us to investigate the effect of a bolus injection on the immobilization behaviour in the tail suspension test in C57BL6J and CD1 mice. All the mice treated with T2 prior to the first test session displayed shortened time spent immobile on both days of testing. Our results, obtained in two treatment protocols on two mouse strains, suggest an antidepressant like effect of used here dosing with T2. Present data with T2 are in accordance with our previous observations that have shown more pronounced antidepressant effects of acutely injected SSRIs and tricyclics, which are administrated prior to the first session of the tail suspension experiment, than afterwards, both in the C57BL6J and CD1 mice (Strekalova, unpublished results). In most of the cases, the antidepressant like action of bolus dosing with conventional antidepressants is often detectable in mice only on the second testing session. Here, however, a reduction of a depressive-like behaviour in T2-treated animals was observed on both Day 1 and Day 2 of the experiment.

Next, we found that both C57BL/6J and CD1 mice showed no changes in the parameters of anxiety and locomotion when treated with $\mathrm{T} 2$ at the doses that have elicited a decrease in the immobility time. Namely, in a classical mouse paradigm for anxiety-like behaviour [29, 34], the dark-light box, CD1 mice that were treated with T2 at the dose $750 \mathrm{mcg} / \mathrm{kg}$ or $1500 \mathrm{mcg} / \mathrm{kg}$ did not show any signs of altered parameters of anxiety-like behaviour. In the novel cage, a sensitive paradigm for minor changes in mouse activity [38, 39], CD1 mice that received T2 at the dose $750 \mathrm{mcg} / \mathrm{kg}$ or $1500 \mathrm{mcg} / \mathrm{kg}$ did not display any locomotor alternations; similar results in both tests were obtained in C57BL/6J (data not shown). These rules out potential impediment in the evaluation of the antidepressant-like activity of the treatment with $\mathrm{T} 2$ that might interfere with the changes in emotionality and general locomotor activity.

The antidepressant-like effects of $\mathrm{T} 2$ in the tail suspension paradigm suggest specific brain mechanisms of transport of peripheral T2. Among several 
thyroid hormone transporters, recent studies have identified monocarboxylate transporter 8 (MCT8) molecule as a very active and specific transporter of all thyroid hormones, $\mathrm{T} 4, \mathrm{~T} 3$, and $\mathrm{T} 2$, which transports them with comparable uptake rates at various locations [47] and is highly expressed in hippocampus, olfactory bulb, cerebral cortex, amygdale, and choroid plexus [48]. A hypothesis was derived for the mechanism of the transport of thyroid hormones from the bloodstream to the brain involving thyroid hormone transporter synthesized in choroid plexus and secreted into the cerebrospinal fluid [49]. While several mechanisms of action of T2 can be proposed, including above-discussed effects of this hormone on monoamine and integrin receptors, PI3 K-Akt signaling and TRH, given accumulated evidences concerning eminent effects of $\mathrm{T} 2$ on the mitochondrial respiratory chain [20, 21], biogenesis [17], and calcium and NO signalling [22], they are likely to be due to the enhancement of mitochondrial functions, a validated target of antidepressant therapy [19].

In conclusion, our data further suggest an importance of central thyroid hormone signalling in depressive-like changes that can be targeted to improve available therapy of this syndrome. Our data also provide support for the translation of $\mathrm{T} 2$ enhancing therapy, perhaps in conjunction with conventional therapy, especially when a rapid therapeutic effect is relevant and/or metabolic parameters of the patients are compromised.

\section{Conflict of Interests}

All other authors declare that they have no conflict of interests. KNAW and RFBR are governmental nonprofit agencies funding basic research and had no further role in the study design, the collection, analysis, and interpretation of data, writing of the report, and the decision to submit the paper for publication.

\section{Authors' Contribution}

Nataliia Markova and Anton Chernopiatko equally contributed to this work. 


\section{Acknowledgments}

The authors thank KNAW and RFBR 11-04-1411 for a support of this study, Mr. Matthew Evans and Professor Daniel C. Anthony, Pharmacology Department, Oxford University, for valuable comments and improvements of the language.

\section{References}

[1] G. Pinna, O. Br"odel, T. Visser et al., "Concentrations of seven iodothyronine metabolites in brain regions and the liver of the adult rat," Endocrinology, vol. 143, no. 5, pp. 1789-1800, 2002.

[2] G. Pinna, O. Broedel, M. Eravci et al., "Thyroid hormones in the rat amygdala as common targets for antidepressant drugs, mood stabilizers, and sleep deprivation," Biological Psychiatry, vol. 54, no. 10, pp. 1049-1059, 2003.

[3] A. Guada no-Ferraz, M. J. Obreg'on, D. L. St Germain, and J. Bernal, "The type 2 iodothyronine deiodinase is expressed primarily in glial cells in the neonatal rat brain," Proceedings of the National Academy of Sciences of the United States of America, vol. 94, no. 19, pp. 10391-10396, 1997.

[4] M. J. Obreg'on, C. R. de Ona, R. Calvo, F. E. del Rey, and G. Morreale de Escobar, "Outer ring iodothyronine deiodinases and thyroid hormone economy: responses to iodine deficiency in the rat fetus and neonate," Endocrinology, vol. 129, no. 5, pp. 2663-2673, 1991.

[5] F. Goglia, "Biological effects of 3,5-diiodothyronine (T2)," Biochemistry, vol. 70, no. 2, pp. 164-172, 2005.

[6] R. T. Joffe, "Is the thyroid still important in major depression?" Journal of Psychiatry and Neuroscience, vol. 31, no. 6, pp. 367-368, 2006.

[7] A. G. Trentin, "Thyroid hormone and astrocyte morphogenesis," Journal of Endocrinology, vol. 189, no. 2, pp. 189-197, 2006.

[8] L. A. Desouza, U. Ladiwala, S. M. Daniel, S. Agashe, R. A. Vaidya, and V. A. Vaidya, "Thyroid hormone regulates hippocampal neurogenesis in the adult 
rat brain,"Molecular and Cellular Neuroscience, vol. 29, no. 3, pp. 414-426, 2005.

[9] T. Giordano, J. B. Pan, D. Casuto, S. Watanabe, and S. P. Arneric, "Thyroid hormone regulation of NGF, NT-3 and BDNF RNA in the adult rat brain," Molecular Brain Research, vol. 16, no. 3-4, pp. 239-245, 1992.

[10] X. Moreau, R. Jeanningros, and P. Majjola-Pomietto, "Chronic effects of triiodothyronine in combination with imipramine on 5-HT transporter, 5HT1A and 5-HT2A receptors in adult rat brain," Neuropsychopharmacology, vol. 24, no. 6, pp. 652-662, 2001.

[11] V. A. Vaidya, M. E. Castro, Q. Pei, M. E. Sprakes, and D. G. GrahameSmith, "Influence of thyroid hormone on 5-HT(1A) and 5-HT(2A) receptormediated regulation of hippocampal BDNF mRNA expression," Neuropharmacology, vol. 40, no. 1, pp. 48-56, 2001.

[12] A. J. Cleare, A. McGregor, and V. O'Keane, "Neuroendocrine evidence for an association between hypothyroidism, reduced central 5-HT activity and depression," Clinical Endocrinology, vol. 43, no. 6, pp. 713-719, 1995.

[13] G. Abraham, R. Milev, and J. Stuart Lawson, "T3 augmentation of SSRI resistant depression," Journal of Affective Disorders, vol. 91, no. 2-3, pp. 211-215, 2006.

[14] M. Bauer, A. Heinz, and P. C. Whybrow, "Thyroid hormones, serotonin and mood: of synergy and significance in the adult brain," Molecular Psychiatry, vol. 7, no. 2, pp. 140-156, 2002.

[15] M. Eravci, G. Pinna, H. Meinhold, and A. Baumgartner, "Effects of pharmacological and non-pharmacological treatments on thyroid hormone metabolism and concentrations in rat brain," Endocrinology, vol. 141, no. 3, pp. 1027-1040, 2000.

[16] A. Antonelli, P. Fallahi, S. M. Ferrari et al., "3,5-diiodo-Lthyronine increases resting metabolic rate and reduces body weight without undesirable side effects," Journal of Biological Regulators and Homeostatic Agents, vol. 25, no. 4, pp. 655-660, 2011. 
[17] P. de Lange, F. Cioffi, R. Senese et al., "Nonthyrotoxic prevention of dietinduced insulin resistance by 3,5-diiodo-L-thyronine in rats," Diabetes, vol. 60, no. 11, pp. 2730-2739, 2011.

[18] M. Moreno, E. Silvestri, R. de Matteis et al., "3,5-diiodo-Lthyronine prevents high-fat-diet-induced insulin resistance in rat skeletal muscle through metabolic and structural adaptations," The FASEB Journal, vol. 25, no. 10, pp. 3312-3324, 2011.

[19] G. T. Rezin, G. Amboni, A. I. Zugno, J. Quevedo, and E. L. Streck, "Mitochondrial dysfunction and psychiatric disorders," Neurochemical Research, vol. 34, no. 6, pp. 1021-1029, 2009.

[20] A. Lombardi, A. Lanni, M. Moreno, M. D. Brand, and F. Goglia, "Effect of 3,5-di-iodo-L-thyronine on the mitochondrial energy-transduction apparatus," Biochemical Journal, vol. 330, part 1, pp. 521-526, 1998.

[21] A. Lanni, M. Moreno, M. Cioffi, and F. Goglia, "Effect of 3,3'-diiodothyronine and 3,5-di-iodothyronine on rat liver mitochondria," Journal of Endocrinology, vol. 136, no. 1, pp. 59-64, 1993.

[22] A. Del Viscovo, A. Secondo, A. Esposito, F. Goglia, M. Moreno, and L. M. Canzoniero, "Intracellular and plasma membrane initiated pathways involved in the [ $\mathrm{Ca} 2+]$ i elevations induced by iodothyronines (T3 and T2) in pituitary GH3 cells," The American Journal of Physiology-Endocrinology and Metabolism, vol. 302, no. 11, pp. E1419-E1430, 2012.

[23] S. G. Ball, J. Sokolov, and W. W. Chin, "3,5-diiodo-L-thyronine (T2) has selective thyromimetic effects in vivo and in vitro," Journal of Molecular Endocrinology, vol. 19, no. 2, pp. 137-147, 1997.

[24] J. C. Strum, R. Shehee, D. Virley et al., "Rosiglitazone induces mitochondrial biogenesis in mouse brain," Journal of Alzheimer's Disease, vol. 11, no. 1, pp. 45-51, 2007.

[25] Y. Zhao, A. Patzer, T. Herdegen, P. Gohlke, and J. Culman, "Activation of cerebral peroxisome proliferator-activated receptors gamma promotes neuroprotection by attenuation of neuronal cyclooxygenase-2 
overexpression after focal cerebral ischaemia in rats," FASEB Journal, vol. 20, no. 8, pp. 1162-1175, 2006.

[26] A. A. Eissa Ahmed and N.M. Al-Rasheed, "Antidepressant-like effects of rosiglitazone, a PPAR? agonist, in the rat forced swim and mouse tail suspension tests," Behavioural Pharmacology, vol. 20, no. 7, pp. 635-642, 2009.

[27] N. L. Rasgon, H. A. Kenna, K. E. Williams, B. Powers, T. Wroolie, and A. F. Schatzberg, "Rosiglitazone add-on in treatment of depressed patients with insulin resistance: a pilot study," TheScientificWorldJOURNAL, vol. 10, pp. 321-328, 2010.

[28] D. E. Kemp, F. Ismail-Beigi, S. J. Ganocy et al., "Use of insulin sensitizers for the treatment of major depressive disorder: a pilot study of pioglitazone for major depression accompanied by abdominal obesity," Journal of Affective Disorders, vol. 136, no. 3, pp. 1164-1173, 2011.

[29] B. H. Cline, H. W. Steinbusch, D. Malin et al., "The neuronal insulin sensitizer dicholine succinate reduces stress-induced depressive traits and memory deficit: possible role of insulin like growth factor 2,"BMCNeuroscience, vol. 13, article 110, 2012.

[30] T. Strekalova, Y. Couch, N. Kholod et al., "Update in the methodology of the chronic stress paradigm: internal control matters," Behavioural and Brain Functions, vol. 7, article 9, 2011.

[31] T. Strekalova and H. M. W. Steinbusch, "Updated methodology of the chronic stress depression model: importance of internal control," in Proceedings of the International Neuroscince Symposiumof the Champalimaud Foundation, Lisbon, Portugal, September 2011.

[32] J. F. Cryan, C. Mombereau, and A. Vassout, "Thetail suspension test as a model for assessing antidepressant activity: review of pharmacological and genetic studies in mice," Neuroscience and Biobehavioural Reviews, vol. 29, no. 4-5, pp. 571-625, 2005. 
[33] M. Moreno, A. Lombardi, L. Beneduce et al., "Are the effects of T3 on resting metabolic rate in euthyroid rats entirely caused by T3 itself?" Endocrinology, vol. 143, no. 2, pp. 504-510, 2002.

[34] Y. Couch, D. C. Anthony, O. Dolgov et al., "Microglial activation, increased TNF and SERT expression in the prefrontal cortex define stressaltered behaviour in mice susceptible to anhedonia," Brain, Behaviour, and Immunity, vol. 29, pp. 136-146, 2013.

[35] T. Strekalova, R. Spanagel, D. Bartsch, F. A. Henn, and P. Gass, "Stressinduced anhedonia in mice is associated with deficits in forced swimming and exploration," Neuropsychopharmacology, vol. 29, no. 11, pp. 20072017, 2004.

[36] E. Malatynska, H. W. Steinbusch, O. Redkozubova et al., "Anhedonic-like traits and lack of affective deficits in 18-month-old C57BL/6 mice: implications for modeling elderly depression," Experimental Gerontology, vol. 47, no. 8, pp. 552-564, 2012.

[37] M. W. Pfaffl, "A new mathematical model for relative quantification in real-time RT-PCR," Nucleic Acids Research, vol. 29, no. 9, article e45, 2001.

[38] J. Vignisse, H.W. M. Steinbusch, A. Bolkunov et al., "Dimebon enhances hippocampus-dependent learning in both appetitive and inhibitory memory tasks in mice," Progress in Neuro-Psychopharmacology and Biological Psychiatry, vol. 35, no. 2, pp. 510-522, 2011.

[39] T. Strekalova, D. C. Anthony, O. Dolgov et al., "The differential effects of chronic imipramine or citalopram administration on physiological and behavioural outcomes in naive mice," Behavioural Brain Research, vol. 245, pp. 101-106, 2013.

[40] P. Willner, "Chronic mild stress (CMS) revisited: consistency and behavioural-neurobiological concordance in the effects of CMS," Neuropsychobiology, vol. 52, no. 2, pp. 90-110, 2005. 
[41] M. Hamilton, "Development of a rating scale for primary depressive illness," The British Journal of Social and Clinical Psychology, vol. 6, no. 4, pp. 278-296, 1967.

[42] J. Harro, M. Tonissaar, M. Eller, A. Kask, and L. Oreland, “Chronic variable stress and partial $5-\mathrm{HT}$ denervation by parachloroamphetamine treatment in the rat: effects on behaviour

and monoamine neurochemistry," Brain Research, vol. 899, no. 1-2, pp. 227239, 2001.

[43] T. Strekalova, N. Gorenkova, E. Schunk, O. Dolgov, and D. Bartsch, "Selective effects of citalopram in a mouse model of stress-induced anhedonia with a control for chronic stress," Behavioural Pharmacology, vol. 17, no. 3, pp. 271-287, 2006.

[44] Y. Ibarguen-Vargas, A. Surget, P. Vourc'h et al., "Deficit in BDNF does not increase vulnerability to stress but dampens antidepressant-like effects in the unpredictable chronic mild stress," Behavioural Brain Research, vol. 202, no. 2, pp. 245-251, 2009.

[45] V. A. Galton, E. T. Wood, E. A. St Germain et al., "Thyroid hormone homeostasis and action in the type 2 deiodinase deficient rodent brain during development," Endocrinology, vol. 148, no. 7, pp. 3080-3088, 2007.

[46] M. Castillo, J. A. Hall, M. Correa-Medina et al., "Disruption of thyroid hormone activation in type 2 deiodinase knockout mice causes obesity with glucose intolerance and liver steatosis only at thermoneutrality," Diabetes, vol. 60,no. 4, pp. 1082-1089, 2011.

[47] E. C. Friesema, S. Ganguly, A. Abdalla, J. E. Manning Fox, A. P. Halestrap, and T. J. Visser, "Identification of monocarboxylate transporter 8 as a specific thyroid hormone transporter," The Journal of Biological Chemistry, vol. 278, no.41, pp.40128-40135, 2003.

[48] L. M. Roberts, K. Woodford, M. Zhou et al., "Expression of the thyroid hormone transporters monocarboxylate transporter-8 (SLC16A2) and 
organic ion transporter-14 (SLCO1C1) at the blood-brain barrier," Endocrinology, vol. 149, no. 12, pp. 6251-6261, 2008.

[49] G. Schreiber, A. R. Aldred, A. Jaworowski, C. Nilsson, M. G. Achen, and M. B. Segal, "Thyroxine transport from blood to brain via transthyretin synthesis in choroid plexus," The American Journal of Physiology, vol. 258, no. 2, part 2, pp. R338-R345, 1990.

\section{Figure legends}

Figure 1: Schematic timeline of the chronic stress (a) and tail suspension ((b)-(d)) experiments.

Figure 2: Chronic stress induces physiological and behavioural changes in a subgroup of anhedonic mice, which are not evident in mice resilient to stress. (a) A loss of sucrose preference was used to define the susceptible group, which was measured after the termination of a 10-day stress paradigm. (b) Latency to immobility in the tail suspension test was significantly shorter in stressed drug-naive animals than in control and stressed imipramine-treated groups. (c)The duration of immobility in the tail suspension test was significantly longer in stressed drug-naive than in control and stressed imipramine-treated groups. (d) In comparison to control mice, the score of coat state was significantly decreased in stressed non treated mice and, to lesser extent, in stressed imipramine-treated animals. (e) Body weight was significantly reduced in both stressed group; it was significantly higher in imipramine-treated than in non treated stressed groups. Data are mean \pm SEM; ${ }^{*} p<0.05,{ }^{* *} p<0.01$, and ${ }^{* * *} p<0.001$; TST: tail suspension test.

Figure 3: Hippocampal gene expression of deiodinases $\mathbf{2}$ and $\mathbf{3}$ in a chronic stress depression model. Illumina assay has pointed to (a) a non-significant decrease in $\mathrm{DIO} 2$ expression in susceptible versus resilient mice and (b) significant elevation of $\mathrm{DIO} 2$ expression in stressed imipramine-treated group in comparison with stressed drug-naive animals. RT-PCR analysis showed a significant (c) decrease in DIO2 expression in susceptible versus resilient mice and (d) increase in $\mathrm{DIO} 2$ expression in stressed imipraminetreated group in comparison with stressed non treated mice. As for $\mathrm{DIO} 3$ 
expression, Illumina assay has revealed no significant differences in its expression between (e) susceptible versus resilient mice and (f) stressed imipramine-treated stressed drug-naive groups. RT-PCR analysis demonstrated a lack of significant differences with the former comparison (g), but (h) showed a significant increase in DIO3 expression in stressed imipramine treated group in comparison with stressed non treated mice $* p<0.05$.

Figure 4: 3,5-diiodo-L-thyronine T2 reduces immobility time in the tail suspension test. On Day 1 and 2 of the tail suspension test, mice treated with $250 \mathrm{mcg} / \mathrm{kg}$ of T2 $30 \mathrm{~min}$ before (a) or $2 \mathrm{~h}$ after (b) the first session of testing showed no differences in the latency of immobility and in the duration of this behaviour. (c) Mice treated with $750 \mathrm{mcg} / \mathrm{kg}$ of T2 $30 \mathrm{~min}$ prior to the first test session had a trend towards longer latency of immobility on Day 1 and significant increase in this parameter on Day 2; the duration of immobility was significantly reduced. (d) Animals that received the dose of $750 \mathrm{mcg} / \mathrm{kg}$ of T2 $2 \mathrm{~h}$ after the first session of testing had significant increase in the latency of immobility on Day 2, and the duration of immobility had a tendency to a decrease. On Day 1, prior to treatment, mice from two groups had similar behavioural scores. (e) Mice dosed with 1500 $\mathrm{mcg} / \mathrm{kg}$ of T2 $30 \mathrm{~min}$ prior to the first test session had a trend towards longer latency of immobility on Days 1 and 2; the duration of immobility was significantly reduced. ${ }^{*} p<0.05$ versus control.

Figure 5. 3,5-Diiodo-L-thyronine T2-treated mice display unaltered anxietylike and locomotor behaviour. In the dark-light box, mice that were treated with $\mathrm{T} 2$ at the dose $750 \mathrm{mcg} / \mathrm{kg}$ or $1500 \mathrm{mcg} / \mathrm{kg}$ did not differ from vehicletreated mice in (a) the time spent in the lit compartment, (b) latency of exit in the lit area, and (c) number of exits to the lit box. (d) In the novel cage, mice that received T2 at the dose $750 \mathrm{mcg} / \mathrm{kg}$ or $1500 \mathrm{mcg} / \mathrm{kg}$ did not differ from vehicle-treated mice in number of rearings. All groups constituted 9 mice. 
Tail

suspension,

Sucrose scoring of Sucrose preference, social interaction test preference $\square \square$ coat state

Dosing with imipramine, weekly body weight monitoring

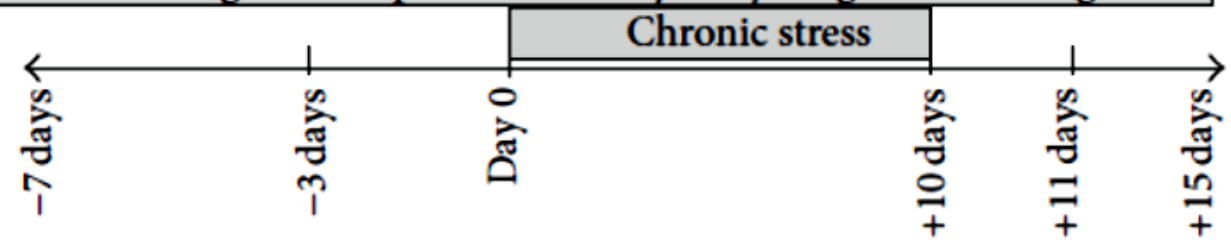

Coat scoring

Tissue collection

(a)

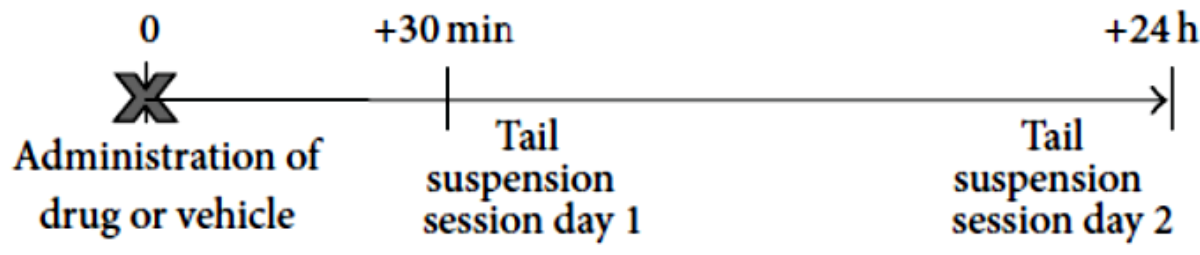

(b)

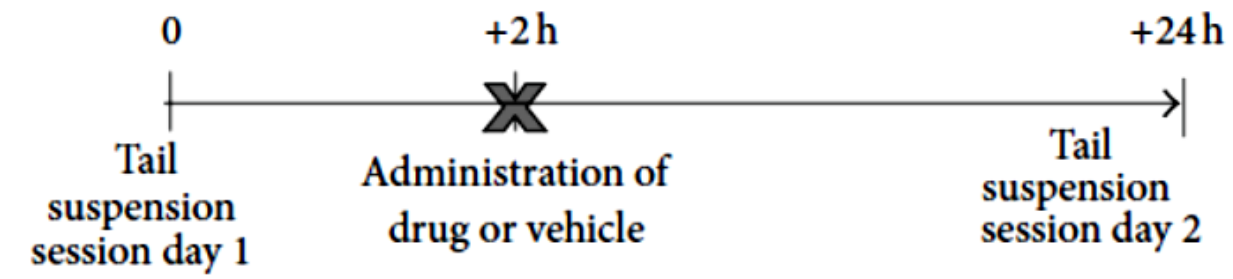

(c)

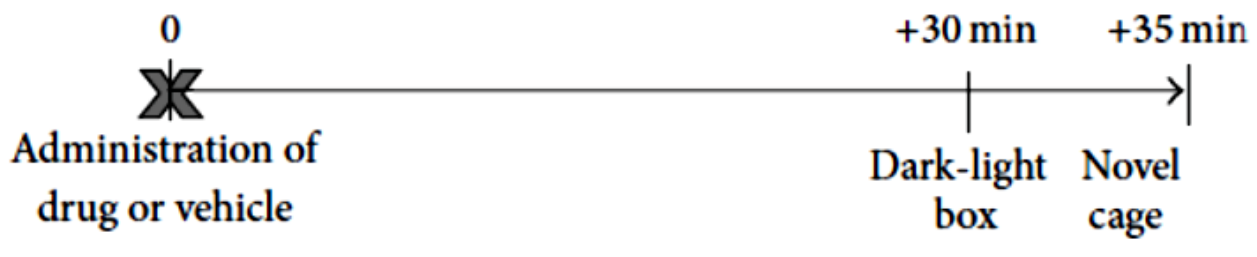

(d)

Figure 1 
A

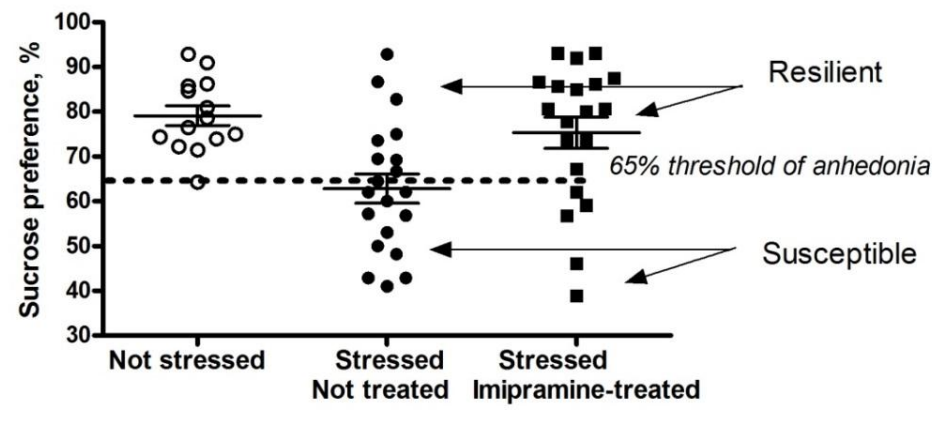

B

TST: Latency to immobility

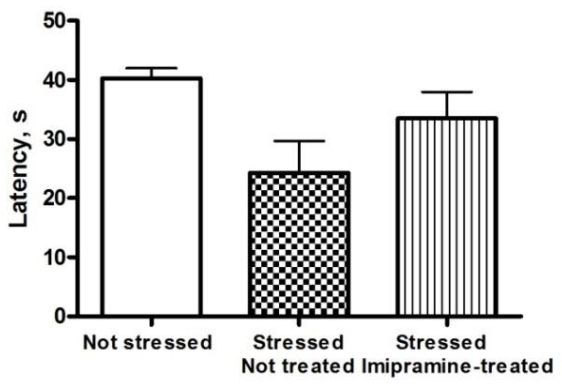

D

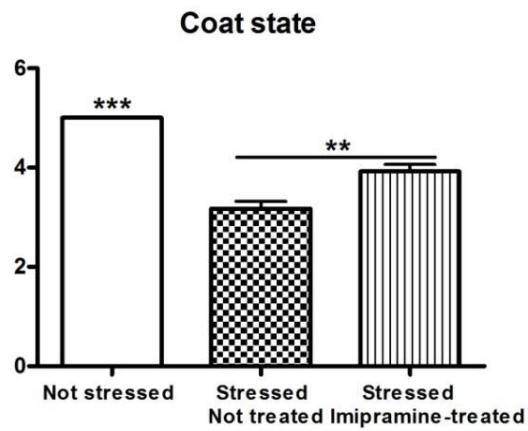

C

TST: Duration of immobility

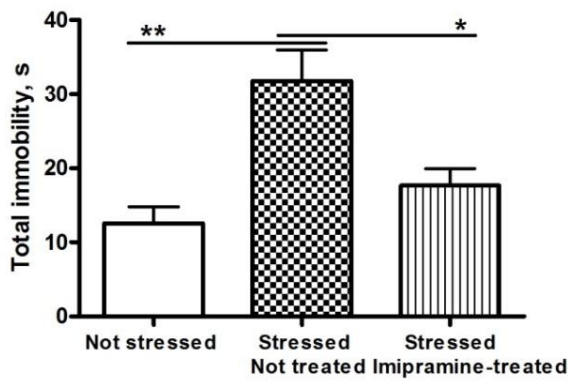

E

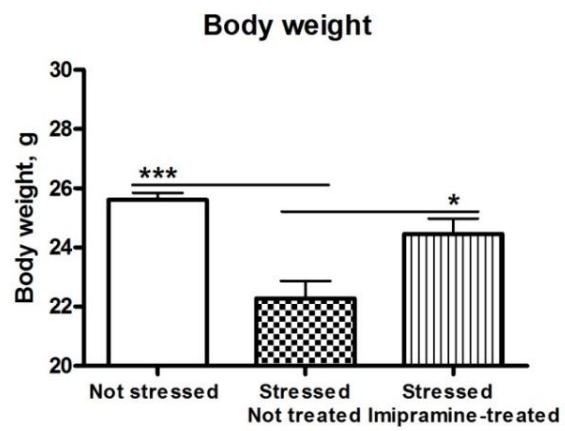

Figure 2 
HIPPOCAMPAL EXPRESSION OF DEIODINASE 2 (DIO2)

Illumina assay
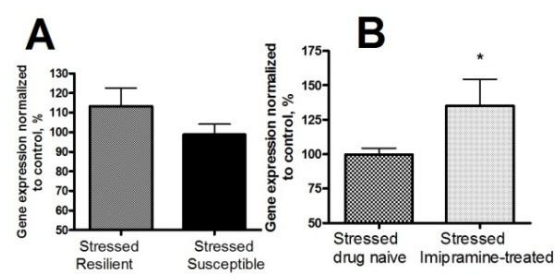

RT PCR

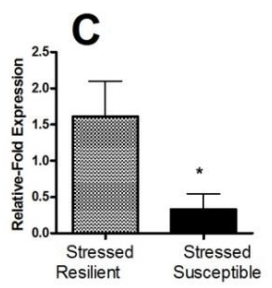

D

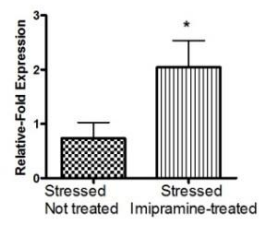

HIPPOCAMPAL EXPRESSION OF DEIODINASE 3 (DIO3)
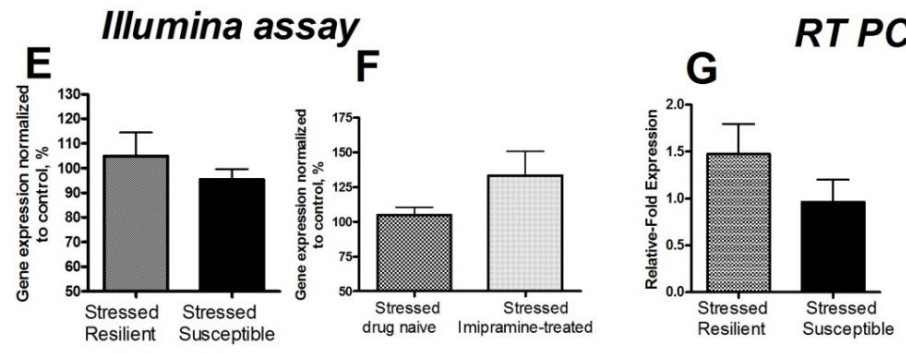

$\mathrm{H}$

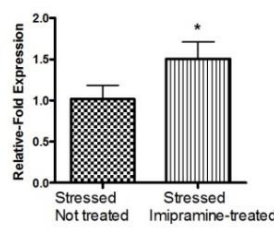

Figure 3 
Dosing with $250 \mathrm{mcg} / \mathrm{kg}$ prior the first TST

Dosing with $250 \mathrm{mcg} / \mathrm{kg}$ after the first TST
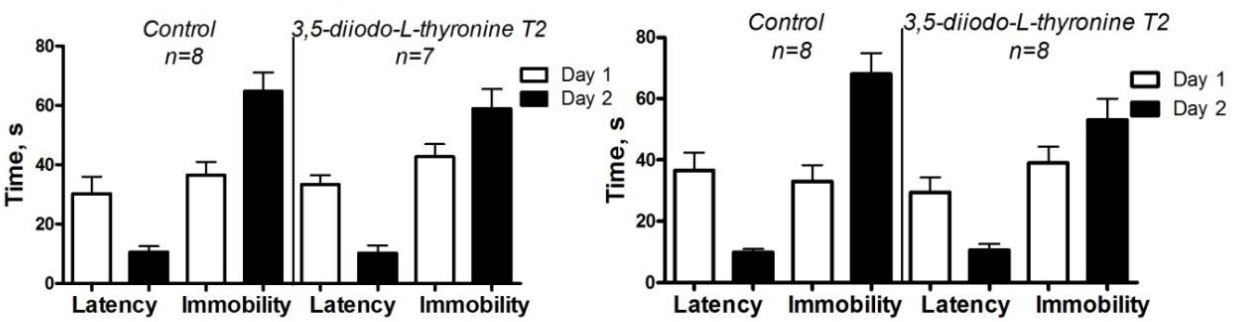

C

D

Dosing with $750 \mathrm{mcg} / \mathrm{kg}$ prior the first TST

Dosing with $750 \mathrm{mcg} / \mathrm{kg}$ after the first TST
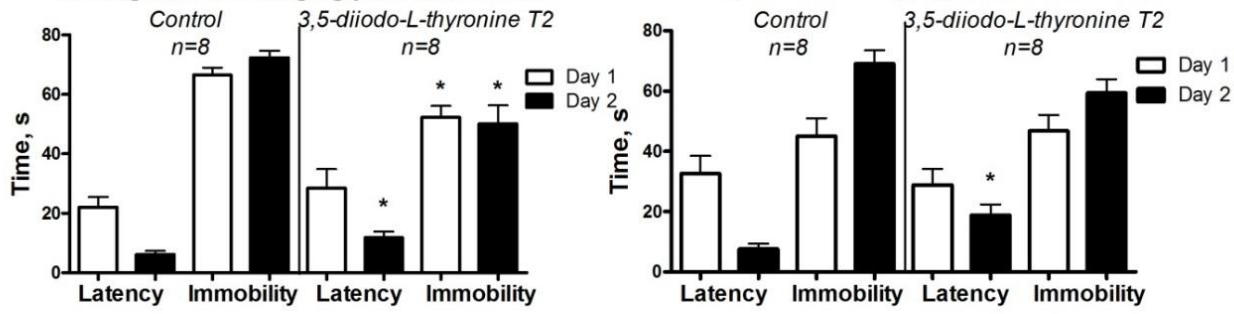

E

Dosing with $1500 \mathrm{mcg} / \mathrm{kg}$ prior the first TST

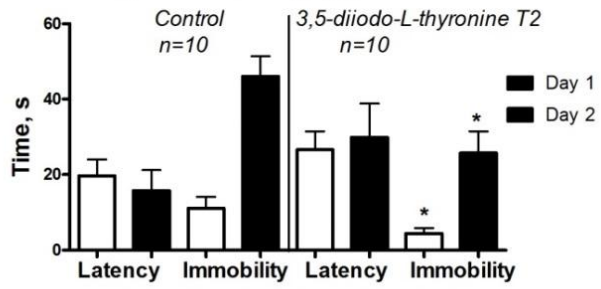

Figure 4 
A
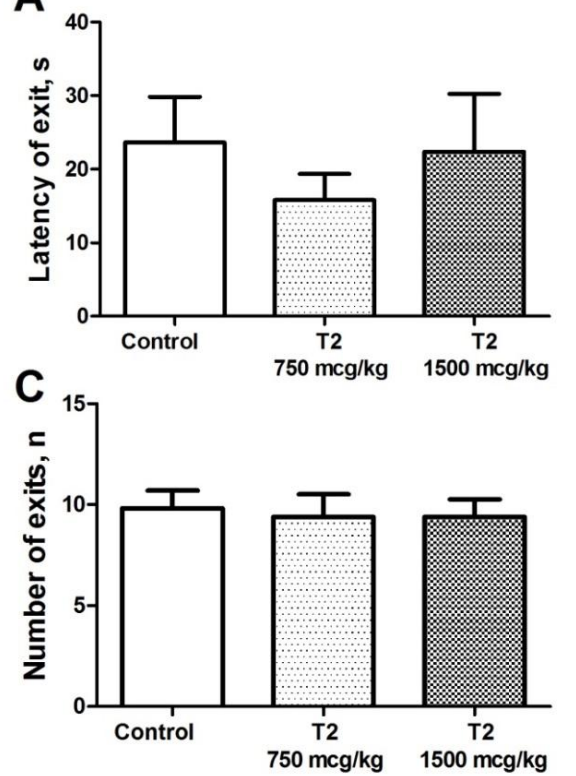

B
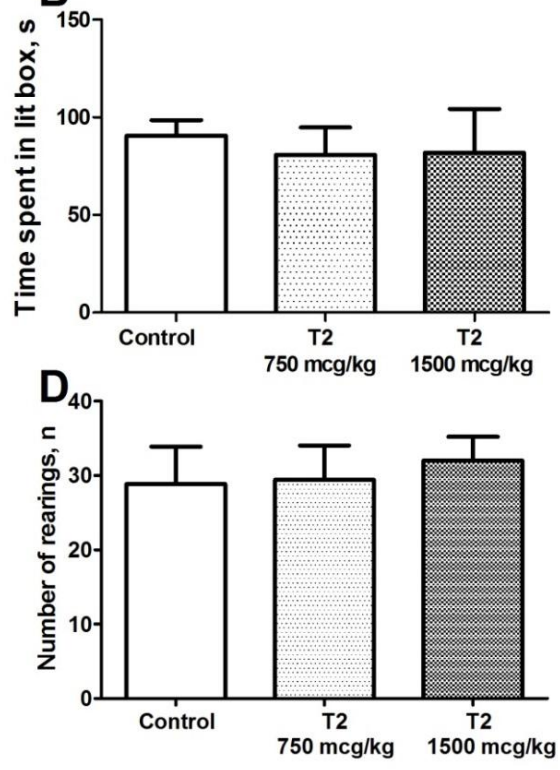

Figure 5 
Chapter 3. A study of the effects of 3,5-diiodo-L-thyronine in the tail suspension and forced swim models of depression

Nataliia Markova ${ }^{1,2,3}$, Anton Chernopiatko ${ }^{4}$, Aslan Kubatiev ${ }^{2}$, Sergey

Bachurin $^{1}$, Harry M. W. Steinbusch ${ }^{3}$, and Tatyana Strekalova ${ }^{3^{*}}$

\begin{abstract}
${ }^{1}$ Institute of Physiologically Active Compounds, Russian Academy of Sciences, Severnii proezd 1, Chernogolovka, Moscow Region 142432, Russia; ${ }^{2}$ Federal State Budgetary Scientific Institution "Institute of General Pathology and Pathophysiology", Baltiyskaia str. 8, Moscow 125315, Russia; ${ }^{3}$ Department of Neuroscience, School for Mental Health and Neuroscience, Maastricht University, Universiteitssingel 40, NL 6229 ER Maastricht, The Netherlands; ${ }^{4}$ Timantti AB, Sundbyberg 104, 17407 Stockholm, Sweden.
\end{abstract}

*Corresponding author Tatyana Strekalova, Ph.D.

t.strekalova@maastrichtuniversity.nl

Markova, N., Chernopiatko, A., Kubatiev, A., Bachurin, S., Steinbusch, $H$. M.W., Strekalova, T., 2016. A study of the effects of 3,5-diiodo-L-thyronine in the tail suspension and forced swim models of depression. Transl. Neurosci. Clin. 2, 96-107. 


\section{Abstract}

Objectives. Clinical studies of the last decade demonstrated beneficial effects of various new adjunctive therapies of depression, including supplementary treatments with thyroid hormones. Recent findings further highlighted the role of the thyroid system in the pathophysiology of this disorder and revealed new physiologically relevant elements of the thyroid system. Our previous study showed an antidepressant-like effect of 3,5-diiodo-Lthyronine (T2), which was earlier considered to be physiologically inactive molecule, in the tail suspension test. Here, we aimed to investigate antidepressant-like effects of T2 hormone further. Methods. We studied the effects of bolus injections of T2 to C57BI6J mice at the doses of 0.25 and 0.75 $\mathrm{mg} / \mathrm{kg}$ that were carried out $30 \mathrm{~min}$ prior the experiment, during two-day testing protocols of the tail suspension and forced swim depression paradigms. Additionally, the effects of the higher dose were investigated in CD1 mice in the forced swim test. Also, potential behavioural effects of these treatments were studied in the novel cage and dark light box tests. Results. A reduction of a depressive-like behaviour, measured by the duration of immobility, was found in mice treated with the dose $75 \mathrm{mg} / \mathrm{kg}$ of $\mathrm{T} 2$ in the tail suspension test, but not in the forced swim test. The parameters of locomotion or anxiety were unaltered by the treatment with T2. We did not find behavioural changes after bolus administration of T2 at the dose of $0.25 \mathrm{mg} / \mathrm{kg}$ in either test for a depressive-like behaviour. Thus, bolus injection of $\mathrm{T} 2$ can induce anti-depressant-like effects without affecting other behaviours. A discrepant result in the forced swim test may be due to its different sensitivity to $\mathrm{T} 2$ as compared to the tail suspension paradigm. Also, the development of procedural modifications of this model can be useful for its application in pre-clinical studies. Conclusions. Given rapid cellular response evoked by $\mathrm{T} 2$ in comparison with the effects of other thyroid hormones, present data suggest clinical benefits from potential application of $\mathrm{T} 2$ as adjuvant therapy of depression, where early onset of therapeutic effect is of particular relevance.

Keywords: forced swim test, tail suspension test, depression, animal models, thyroid hormones. 


\section{Introduction}

Thyroid hormones are important in brain function and dysfunction, including affective disorders and depression. It is well-established that thyroid hormones are essential for crucial processes in the brain and implicated in neuronal processing, proliferation and integration, CNS myelination and the synthesis of key enzymes required for neurotransmitter synthesis, thus, playing important roles in the development and adaptive responses of the nervous system [1, 2]. By now, it is commonly accepted that thyroid hormones are important regulators of the mechanisms, which are relevant to the pathogenesis of affective conditions that is particularly supported by numerous studies demonstrating the efficacy of adjuvant therapy with 3,3,5triiodo-L-thyronine (T3) and 3,5,3',5'-tetraiodo-L-thyronine (T4) thyroid hormones in depressed patients [3-6].

Both T3 and T4 hormones, however, are known to produce a delayed response in a cell $[7,8]$. In contrast to T3 and T3 hormones, 3,5-diiodo-Lthyronine (T2), which has been recently identified as functionally inactive metabolite of T3 in periphery in vivo and in vitro systems, exerts rapid effects on the mammal cells and, as such, might possess unique antidepressant-like properties to be exploited in a clinic [7]. While T3, whose antidepressant-like properties are well documented and known to be more pronounced than that of $\mathrm{T} 4$, evokes the lasting effects of on metabolic rate and mitochondrial respiration, with onset delayed for $48 \mathrm{~h}$. The action of T2 begins within the first few hours and does not last for longer than $48 \mathrm{~h}$ and is not sensitive to actinomycin D $[7,9,10]$. Unlike T3, T2 increases mitochondrial respiration by a nuclear-independent mechanism, which is not realized via thyroid hormone receptor beta, nor via AMP-activated protein kinase. Other physiological activities of $\mathrm{T} 2$ were shown to be distinct from the effects of $T 3$, such as effects on mitochondrial pathways, where T2 was demonstrated to stimulate reactions involved in substrate oxidation $[9,11]$ and increase the downstream mechanisms, which are involved in mitochondrial biogenesis $[12,13]$. Also, T2 was found to evoke selective thyromimetic activity in vitro and in vivo that is divergent from that of T3 [14]. Together, growing evidence suggests that the effects of $T 2$ do not 
merely mimic those of $\mathrm{T} 3$, but, instead, involve discrete mechanisms and that seem likely to have a rapid dynamic.

Antidepressants with fast onset of therapeutic effects are currently on high demand in clinical management of a depressive disorder. Ketamine is a probably a unique example of effective antidepressants of this kind, whose practical use, unfortunately, is largely limited due to serious side effects, such as euphoria, confusion, cognitive impairment or transient dissociative states [15]. In a light of these challenges, studies of potential application of T2 as antidepressant treatment with rapidly developing therapeutic effect can be of high interest, due to its high physiological activity that allows low therapeutical doses to be used, and endogenous origin of this molecule that decreases a risk of occurrence of side effects during a treatment with T2. Meanwhile, up to now, the antidepressant-like effects of T2 are poorly investigated.

The latest evidence suggests that T2 may be important for antidepressant effects. As for instance, chronic treatment with the tricyclic antidepressant desipramine in the rat induces the expression of 12 in the amygdala that is paralleled by elevated levels of T2 expression in the mitochondria, while concentrations of T3 are increased in nuclei [16]. The group of Pinna [16] has also shown the presence of metabolites of T2 in several brain areas, including the structures of the limbic system, such as hippocampus and septum, that points to the occurrence of $\mathrm{T} 2$ itself in these brain areas. Recently, we have demonstrated that mice acutely treated with $\mathrm{T} 2$, display shortened time spent immobile in the two-day tail suspension test, suggesting antidepressant-like effects of $\mathrm{T} 2$ [17].

To study the antidepressant-like effects of $\mathrm{T} 2$ further, we employed the forced swim test, another commonly used paradigm for depressive-like behaviour in mice [18-20]. Like the tail suspension model, the forced swim test is based on an induction of a helplessness and despair behaviour in rodents, which are placed in an inescapable stressful situation for a few minutes $[18 ; 21]$. In the context of these tests, the posture of floating/immobility of tested animals, was originally ascribed by Porsolt to a state of "behavioural despair", that was largely based on the assumption 
that the animals have "given up hope of escaping". It is currently regarded that behavioural responses of animals in these two paradigms manifest an evolutionary preserved coping strategy, where floating/immobility behaviour in animals parallels a human state of "entrapment" and psychomotor impairments during clinical depression, and is due to motivational rather than physical factors [22]. This view is supported by the facts that animals can adopt floating/immobility posture rather quickly and drugs which may suppress activity, e.g., antidepressants, counteract the immobility response [19].

While both tail suspension and forced swim paradigms are regarded to involve similar neurobiological factors and show a substantial overlap in the pharmacological sensitivity to various drugs [23, 24], available literature suggests the differences between these models in the induction / measuring of depressive-like features in tested rodents. These differences were found while comparing the effects of gender differences [25], dosing with SSRIs [26], genetic manipulations [27] and other factors [19, 20, 29].

In the present study, we investigated the antidepressant-like effects of $\mathrm{T} 2$ administrated to C57BI6J mice at the doses of 0.25 and $0.75 \mathrm{mg} / \mathrm{kg}$ in the forced swim and tail suspension tests. Additionally, we used the treatment at the dose of $0.75 \mathrm{mg} / \mathrm{kg}$ of T2 in CD1 mice in the forced swim test. Finally, potential effects of $\mathrm{T} 2$ of above-indicated doses on general behaviour were assessed in the dark-light box anxiety paradigm and a novel cage vertical activity task.

\section{Experimental}

\section{Animals}

Studies were performed using 3-month-old male C57BL/6J and CD1 mice from RAS, Moscow region a provider licensed by Charles River (http://www.spf-animals.ru/about/providers/animals). Mice were single housed under a reversed 12-hour light-dark cycle (lights on: 20:00 h) starting from the day of animals' transportation in the laboratory with food and water provided ad libitum, under controllable laboratory conditions (22 $\pm 1^{\circ} \mathrm{C}, 55 \%$ humidity). All experiments were carried out in accordance with 
the European Communities Council Directive for the care and use of laboratory animals 2010/63/EU.

\section{Experimental design}

We investigated the effects of a bolus oral gavage of T2 to C57BL6J mice at the dose of $0.25 \mathrm{mg} / \mathrm{kg}$ and $0.75 \mathrm{mg} / \mathrm{kg} 30 \mathrm{~min}$ before the first session testing session as described previously [17] in the two-day tail suspension paradigm (Fig. $1 \mathrm{~A}$ ), and in the two-day forced swim model (Fig. $1 \mathrm{~B}$ ). Additionally, CD1 mice were treated with $\mathrm{T} 2$ at the dose of $0.75 \mathrm{mg} / \mathrm{kg}$ via above-described method and tested in the forced swim model. Control mice received a vehicle $30 \mathrm{~min}$ before the first session of tail suspension session or forced swim tests. A selection of doses of $\mathrm{T} 2$ and the timing of dosing were based on our previous results, which showed higher efficacy of treatment, which was carried out 30 min prior the experiment rather than of a treatment performed $2 \mathrm{~h}$ after the first testing session [17]. Additionally, we addressed the question of whether the administration of T2 in investigated doses influences anxiety and locomotion of mice in the darklight box and a novel cage tasks. These experiments were aimed to rule out potential confounds with the evaluation of behavioural despair in the models of depression, after the treatment with T2. Dark-light box and novel cage were applied with a $5 \mathrm{~min}$ interval $30 \mathrm{~min}$ after a dosing (Fig. $1 \mathrm{C}$ ). Except novel cage assay, all behavioural data were normalized to mean values of control group and expressed in percent. The number of mice in each group is indicated in the figure legends, for each experiment.

\section{Tail suspension test}

The protocol used in this study was used as described previously [30]. Mice were subjected to the tail suspension by being hung by their tails with adhesive tape to a rod $50 \mathrm{~cm}$ above the floor for $6 \mathrm{~min}$. The latency of the first episode of immobility, time spent with immobility at 2-min intervals and for entire $6 \mathrm{~min}$ period were scored manually using criteria, which were previously validated by automated scoring with Noldus EthoVision XT 8.5 (Noldus Information Technology, Wageningen, The Netherlands) and CleverSys (CleverSys, Reston, VA, USA) as described elsewhere [24, 30]. The 
immobility behaviour was defined as the absence of any movements of the animals' head and body. The latency of immobility was determined as the time between the onset of the test and the first bout of immobility. This procedure was carried out twice with a $24 \mathrm{~h}$ interval between tests. The trials were recorded by a video camera positioned directly in front of the mice while the experimenter observed the session from a distance in a dark area of the experimental room.

\section{Forced swim test}

Mice were subjected to two swimming sessions spaced $24 \mathrm{~h}$ apart, as previously described [30]. All sessions were 6-min long and were performed by placing a mouse in a transparent cylinder $(\varnothing 17 \mathrm{~cm})$ filled with water $(+23$ ${ }^{\circ} \mathrm{C}$, water height $13 \mathrm{~cm}$, height of cylinder $20 \mathrm{~cm}$ ). The latency of the first episode of floating and time spent with floating at 2-min intervals and for entire $6 \mathrm{~min}$ period was scored manually using criteria, which were previously validated by automated scoring with Noldus EthoVision XT 8.5 (Noldus Information Technology, Wageningen, The Netherlands) and CleverSys (CleverSys, Reston, VA, USA) as described elsewhere [24, 30]. The floating behaviour was defined as the absence of any movements of the animals' head and body. The latency of floating was determined as the time between the onset of the test and the first bout of immobility. The trials were recorded by a video camera positioned directly in front of the mice while the experimenter observed the session from a distance in a dark area of the experimental room.

\section{Dark-light box}

The dark-light box (TechnoSmArt, Rome, Italy) consisted of two plexiglass compartments, one black (dark compartment) $(15 \mathrm{~cm} \times 20 \mathrm{~cm} \times 25 \mathrm{~cm})$ and one white (light compartment) $(30 \mathrm{~cm} \times 20 \mathrm{~cm} \times 25 \mathrm{~cm})$, connected by a tunnel. Anxiety-like behaviour was assessed by earlier validated measures [31]. Mice were placed into the dark compartment, from where they could visit the lit box (light intensity 25 Lux). The latency of the first exit to the light compartment, the total duration of time spent in the lit compartment, and 
the number of visits to this light compartment were scored by visual observation over $5 \mathrm{~min}$.

Novel cage

The novel cage test was performed to assess vertical activity [30, 31]. Mice were introduced into a standard plastic cage; the size of their home cage filled with small amounts of fresh sawdust. The number of exploratory rearings was counted per each minute under red light during a 5-min period. The testing was carried out in a dark quiet room in morning hours.

\section{Statistics}

Data were analyzed with GraphPad Prism version 5.00 for Windows (San Diego, CA). Mann-Whitney test was applied for two-group data sets, as data had no normal distribution. The level of confidence was set at $95 \%(p<0.05)$ and data are normalized to control and shown as mean \pm SEM.

\section{Results}

Effects of T2 at the dose of $0.25 \mathrm{mg} / \mathrm{kg}$ on immobility and floating behaviours

In the tail suspension test, the latency and total duration of immobility in $\mathrm{C} 57 \mathrm{Bl} / 6$ mice dosed with $0.25 \mathrm{mg} / \mathrm{kg}$ of $\mathrm{T} 2$, in comparison to a vehicletreated group, were unaltered on Day $1(p=0.4175$ and $p=0.6425$, respectively, Mann-Whitney test; Fig. $2 A)$ and Day $2(p=0.7711$ and $p=$ 0.3969 , respectively, Mann-Whitney test; Fig. 2 B). As compared with control mice, a group of animals subjected to the administration of $\mathrm{T} 2$ at the dose $0.25 \mathrm{mg} / \mathrm{kg}$ did not show changes in the duration of immobility on Day 1 (1-2 min: $p=0.3533,3-4 \min : p=0.4515$, and $5-6 \min : p=0.4515$, respectively, Mann-Whitney test; Fig. $2 \mathrm{~A}$ ) and Day 2 (1-2 min: $p=0.3969,3-4 \min : p=$ 0.2968 , and 5-6 min: $p=0.8619$, respectively, Mann-Whitney test; Fig. 2 B).

In the forced swim test, in comparison to administration of a vehicle, dosing with $0.25 \mathrm{mg} / \mathrm{kg}$ of T2 did not alter the latency and total duration of floating of $\mathrm{C} 57 \mathrm{Bl} / 6$ mice on Day 1 ( $p=0.8698$, and $p=0.9767$, respectively, Mann- 
Whitney test; Fig. 2 C) and Day $2(p=0.2136$ and $p=0.9031$, respectively, Mann-Whitney test; Fig. 2 D). In comparison to a vehicle-treated mice, there was a tendency to a decreased floating at the second two-minute testing interval of test on Day 1 (2-3 min: $p=0.0771$, Mann-Whitney test; Fig. 2 C). No significant differences between the groups were found in the duration of floating during other periods of observation on Day 1 either (1-2 min: $p=$ 0.3132, and 5-6 min: $p=0.3574$, respectively, Mann-Whitney test; Fig. 2 C) and on Day 2 (1-2 $\min : p=0.3643,3-4 \min : p=0.6846$, and 5-6 $\min : p=$ 0.8497 , respectively, Mann-Whitney test; Fig. 2 D).

\section{Effects of $0.75 \mathrm{mg} / \mathrm{kg}$ of $\mathrm{T} 2$ on immobility and floating behaviours}

In the tail suspension test, bolus administration of T2 at the dose $0.75 \mathrm{mg} / \mathrm{kg}$ did not alter the latency and total duration of immobility of mice, in comparison to injection of a vehicle, on Day $1(p=0.9166$ and $p=0.6905$, respectively, Mann-Whitney test; Fig. $3 A)$ and Day $2(p=0.2812$ and $p=$ 0.2222 , respectively, Mann-Whitney test; Fig. 3 B). A significant decrease of duration of immobility measure in T2-treated versus vehicle-treated animals was found at the first two-minute testing interval of test on Day 1 (1-2 min: $p=0.008$, Mann-Whitney test; Fig. 3 A) and Day 2 (1-2 min: $p=0.0317$, Mann-Whitney test; Fig. 3 B). Immobility behaviour in T2-treated mice was not significantly changed during the remaining periods of scoring, in comparison to a control group, on Day 1 (3-4 min: $p=0.4005$, and 5-6 min: $p$ $=0.2492$, respectively, Mann-Whitney test; Fig. $3 \mathrm{~A}$ ) and Day 2 (3-4 min: $\mathrm{p}=$ 1.000 and 5-6 min: $p=0.6905$, respectively, Mann-Whitney test; Fig. 3 B).

In the forced swim test carried out on $\mathrm{C} 57 \mathrm{BI} / 6$ mice, in comparison to a vehicle-treated group, animals treated with $\mathrm{T} 2$ at the dose $0.75 \mathrm{mg} / \mathrm{kg}$, showed unaltered latency of floating on Day 1 ( $p=0.9360$ Mann-Whitney test, Fig. 3 C) and Day 2 ( $p=0.5738$, Mann-Whitney test; Fig. 3 D). As compared to controls, pharmacologically treated mice exhibited a tendency to a decrease of total duration of floating on Day 1 ( $p=0.0777$, MannWhitney test; Fig. $3 \mathrm{C}$ ), but not on Day 2 ( $p=0.9372$, Mann-Whitney test; Fig. $3 \mathrm{D}$ ). The administration of $\mathrm{T} 2$ at the dose $0.75 \mathrm{mg} / \mathrm{kg}$ did not affect the duration of floating of mice at any two-minute testing intervals, as compared to injection of vehicle, on Day 1 (1-2 $\min : p=0.8089,3-4 \min : p=0.3939$, 
and 5-6 min: $p=0.1087$, respectively, Mann-Whitney test; Fig. 3 C). Similarly, on Day 2, no significant changes were found in the duration of floating between T2- and vehicle-treated groups (1-2 $\min : p=0.8182,3-4 \min : p=$ 1.000 , and 5-6 min: $p=0.6304$, respectively, Mann-Whitney test; Fig. 3 D) of the test.

In the forced swim test carried out on CD1 mice, as compared to vehicletreated group, mice treated with $\mathrm{T} 2$ at the dose $0.75 \mathrm{mg} / \mathrm{kg}$, showed unchanged latency and total duration of floating on Day 1 ( $p=0.8092$ and $p$ $=0.4848$, respectively, Mann-Whitney test; Fig. $3 \mathrm{E})$ and Day $2(p=0.6858$ and $p=0.2403$, respectively, Mann-Whitney test; Fig. 3 F). In comparison to controls, no changes of floating was found in T2-treated mice during selected time intervals on Day 1 (1-2 min: $p=0.3776,3-4 \min : p=0.7483$, and 5-6 min: $p=0.2290$, respectively, Mann-Whitney test; Fig. 3 E). CD1 mice treated with $\mathrm{T} 2$, as compared with vehicle-treated animals, had a tendency to a decreased floating at the first two-minute testing interval of the est on Day 2 (1-2 min: $p=0.0651$, Mann-Whitney test; Fig. 3 F), but not during the last testing intervals (3-4 min: $p=0.2971$ and 5-6 min: $p=0.2403$, respectively, Mann-Whitney test; Fig. 3 F).

\section{T2-treated mice display unaltered anxiety and locomotor behaviour}

Dosing of $\mathrm{C} 57 \mathrm{Bl} / 6$ mice with $\mathrm{T} 2$ at the dose of $0.75 \mathrm{mg} / \mathrm{kg}$ did not alter anxiety-like behaviour of mice, as shown by unaffected latencies of the first exit to the lit compartment in dosed animals, in comparison to a vehicletreated group ( $p=0.4059$, Mann-Whitney test; Fig. 4 A). No significant differences were found between these groups in time spent therein $(p=$ 0.5830, Mann-Whitney test), and number of exits ( $p=0.7112$, MannWhitney test; Fig. 4 B, C). In the novel cage, C57BI/6 mice that received $\mathrm{T} 2$ at the dose $0.25 \mathrm{mg} / \mathrm{kg}$ and CD1 mice treated with $T 2$ at the dose $0.75 \mathrm{mg} / \mathrm{kg}$ did not differ from vehicle-treated mice in a number of rearings at no time point of scoring $(0.25 \mathrm{mg} / \mathrm{kg}: 1 \mathrm{~min}: \mathrm{p}=0.9095,2 \mathrm{~min}: \mathrm{p}=0.6201,3 \mathrm{~min}: \mathrm{p}=$ $0.3423,4 \min : p=0.5188,5 \min : p=0.9696$ and $0.75 \mathrm{mg} / \mathrm{kg}: 1 \mathrm{~min}: p=$ $0.9271,2 \min : p=0.5219,3 \min : p=0.4286,4 \min : p=0.3120,5 \min : p=$ 0.0948, Mann-Whitney test; Fig. 4 D, E). 


\section{Discussion}

In line with previous results, the dosing with T2 at the dose $0.75 \mathrm{mg} / \mathrm{kg}$ has decreased depressive-like behaviour measured by the duration of immobility, in the tail suspension test, during the first minutes of testing [17]. However, no changes in the parameters of floating behaviour in the forced swim test were observed in mice that received the treatment which was applied in the tail suspension test. A lack of significant behavioural effects of T2 in the forced swim test was evidenced both in C57BI/6 and CD1 mouse strains. The administration of $\mathrm{T} 2$ did not change the parameters of anxiety and vertical locomotor activity, suggesting that general behavioural activity of mice was not altered by $\mathrm{T} 2$ and reported changes in immobility are unlikely to be due to its general effects on locomotion. The administration of $\mathrm{T} 2$ at the dose of $0.25 \mathrm{mg} / \mathrm{kg}$ did not induce any effects in either paradigm.

Discrepant effects of compounds with antidepressant activity in the tail suspension and forced swim test are not uncommon in the literature. For example, administration of tricyclic antidepressant imipramine was shown to decrease immobility in the tail suspension test at the dose $4 \mathrm{mg} / \mathrm{kg}$, while in the forced swim test, the dose of $30 \mathrm{mg} / \mathrm{kg}$ of imipramine was effective [32]. Studies with SSRI antidepressant reboxetine revealed a decrease of floating behaviour in the forced swim test, but not in the tail suspension test [26]. Several other reports evidenced discrepant effects of antidepressant in two depression paradigms [23].

A difference in the behavioural changes after T2 administration in the present study may be due to the factor of hypothermia in the forced swim test, which can interfere with metabolic effects of T2. Given rapid effects of T2 on the mitochondrial activation, it can be hypothesized that this treatment counteracts hypothermic effect of swimming procedure that can decrease floating in small rodents $[7,33]$. Other drugs, including imipramine, are known to produce the opposing to T2 effect, decreasing body temperature and potentiating swimming in rodents and thus, thermogenesis, that helps a physiological adaptation to a cold. The hypothermia is considered as one of the major factor affecting rodent's 
behaviour subjected to a forced swimming during Porsolt test [32], where pharmacological treatments, particularly, with tricyclic antidepressants, might play a role [33]. As for instance, independent studies have shown that the effects of amitryptiline and desipramine on floating behaviour can be detectable or more pronounced when the water in the tank is warm rather than cool [33].

Moreover, floating and immobility behaviours observed in small rodents during repeated tail suspension and forced swim tests can also implicate the elements of contextual learning. As such, drugs or other challenges which interfere with this form of memory may affect behavioural read-outs in these models [34]. Among two paradigms, the impact of contextual learning is regarded to be somewhat higher in the forced swim test, because of generally more stressful effects of the procedure. However, given the fact that generally thyroid hormones are required for normal neuronal plasticity and learning, and their exogenous supply can improve cognition, it is unlikely that this factor test can explain the difference between the tail suspension and forced swim experiments with $\mathrm{T} 2$.

In a broader context, floating and immobility behaviours induced in rodents in two employed here depression paradigms can play distinct roles in the behavioural repertoire of animals and be affected differentially by various challenges. For example, parallels have being made between these behaviours and the freezing behaviour following exposure to aversive stimuli, such as a predator and a shock, or the environmental context where the stressful experience was previously taking place [35]. As such, floating and immobility behaviours displayed by animals may depend on immediate adverse effects of external stressors. Taking into account rather weak antidepressant-like effect of $\mathrm{T} 2$ reported in this and previous studies with the tail suspension test, one can suggest that a lack of antidepressant-like changes in the forced swim test was due to higher stress impact of the latter paradigm. A tendency to a significant effect of T2 at the dose $0.75 \mathrm{mg} / \mathrm{kg}$ suggests a possibility that antidepressant-like action of T2 could likely occur under modified conditions of testing in the forced swim model. 
Present study further suggests that floating and immobility behaviours are complex and their accurate analysis requires a detailed assessment. As for instance, our results suggest that scoring of depressive-like behaviours in these tests by short time intervals can increase the accuracy of the analysis. We repeatedly showed that the first 2-min interval of scoring of a depressive-like behaviour can be more sensitive in both tests, rather than frequently used analysis of entire 6 -min observation period. Finally, a development of methodological variants of these tests can be of use in their pre-clinical application. In particular, given present and other facts of limited sensitivity of the forced swim test with the analysis of the antidepressant effects in small rodents, one can suggest that a modification of existing protocols of this paradigm could be potentially beneficial for the neurobiological and translational studies on depression which broadly use this model $[19,23,36]$.

\section{Acknowledgments}

The reported study was partially supported by RFBR, research project No. 16-34-01165.

\section{References}

[1]. Porterfield SP, Hendrich CE. The role of thyroid hormones in prenatal and neonatal neurological development-current perspectives. Endocr Rev 1993, 14 (1): 94-106.

[2]. Bernal J, Nunez J. Thyroid hormones and brain development. EurJ Endocrinol 1995, 133 (4): 390-398.

[3]. Baumgartner A. Thyroxine and the treatment of affective disorder: An overview of the results of basic and clinical research. Int J Neuropsychopharmacol 2000, 3 (2): 149-165.

[4]. Moreau X, Jeanningros R, Majjola-Pomietto P. Chronic effects of triiodothyronine in combination with imipramine on 5-HT transporter, 5$\mathrm{HT}(1 \mathrm{~A})$ and $5-\mathrm{HT}$ (2A) receptors in adult rat brain. Neuropsychopharmacology 2001, 24 (6): 652-662. 
[5]. Bauer M1, Heinz A, Whybrow PC. Thyroid hormones, serotonin and mood: of synergy and significance in the adult brain. Mol Psychiatry 2002, 7 (2): 140-156.

[6]. Abraham G, Milev R, Stuart Lawson J. T3 augmentation of SSRI resistant depression. J Affect Disord 2006, 91 (2-3): 211-215.

[7]. Goglia F. Biological effects of 3,5-diiodothyronine (T(2)). Biochemistry (Mosc) 2005, 70 (2): 164-172.

[8]. Schroeder AC, Privalsky ML. Thyroid hormones, T3 and T4, in the brain. Front Endocrinol (Lausanne) 2014, 5 (40).

[9]. Lombardi A, Lanni A, Moreno M, Brand MD, Goglia F. Effect of 3,5-diiodo-L-thyronine on the mitochondrial energy-transduction apparatus. Biochem J 1998, 330 (1): 521-526.

[10]. Moreno M, Lombardi A, Beneduce L, Silvestri E, Pinna G, Goglia F, Lanni $A$. Are the effects of $\mathrm{T} 3$ on resting metabolic rate in euthyroid rats entirely caused by T3 itself? Endocrinology 2002, 143 (2): 504-510.

[11]. Lanni A, Moreno M, Cioffi M, Goglia F. Effect of 3,3'-di-iodothyronine and 3,5-di-iodothyronine on rat liver mitochondria. J Endocrinol 1993, 136 (1): 59-64.

[12]. deLange P, Cioffi F, Senese R, Moreno M, Lombardi A, Silvestri E, et al. Nonthyrotoxic prevention of diet-induced insulin resistance by 3,5-diiodoL-thyronine in rats. Diabetes 2011, 60 (11): 2730-2739.

[13]. Del Viscovo A, Secondo A, Esposito A, Goglia F, Moreno M, Canzoniero LM. Intracellular and plasma membrane-initiated pathways involved in the [ $\mathrm{Ca} 2+]$ i elevations induced by iodothyronines (T3 and $\mathrm{T} 2$ ) in pituitary GH3 cells. Am J Physiol Endocrinol Metab 2012, 302 (11): 14191430.

[14]. Ball SG, Sokolov J, Chin WW. 3,5-Diiodo-L-thyronine (T2) has selective thyromimetic effects in vivo and in vitro. J MolEndocrinol 1997, 19 (2): 137147.

[15]. Zarate CA Jr, Singh JB, Carlson PJ, Brutsche NE, Ameli R, Luckenbaugh DA, Charney DS, Manji HK. A randomized trial of an N-methyl-D-aspartate antagonist in treatment-resistant major depression. Arch Gen Psychiatry 2006, 63 (8): 856-864.

[16]. Pinna G, Broedel O, Eravci M, Stoltenburg-Didinger G, Plueckhan H, Fuxius $\mathrm{S}$, et al. Thyroid hormones in the rat amygdala as common targets for 
antidepressant drugs, mood stabilizers, and sleep deprivation. Biol Psychiatry 2003, 54 (10): 1049-1059.

[17]. Markova N, Chernopiatko A, Schroeter CA, Malin D, Kubatiev A, Bachurin S, Costa-Nunes J, Steinbusch HM, Strekalova T. Hippocampal gene expression of deiodinases 2 and 3 and effects of 3,5-diiodo-L-thyronine T2 in mouse depression paradigms. Biomed Res Int 2013, 565218.

[18]. Porsolt RD, Bertin A, Jalfre M. Behavioural despair in mice: a primary screening test for antidepressants. Arch Int Pharmacodyn Ther 1977, 229 (2): 327-336.

[19]. Lucki I. The forced swimming test as a model for core and component behavioural effects of antidepressant drugs. Behav Pharmacol 1997, 8 (6-7): 523-532.

[20]. Cryan JF1, Markou A, Lucki I. Assessing antidepressant activity in rodents: recent developments and future needs. Trends Pharmacol Sci 2002, 23 (5): 238-245.

[21]. Steru L, Chermat R, Thierry B, Simon P. The tail suspension test: a new method for screening antidepressants in mice. Psychopharmacology (Berl) 1985, 85 (3): 367-370.

[22]. Weingartner $H$, Silberman E. Models of cognitive impairment: cognitive changes in depression. Psychopharmacol Bull 1982, 18 (2): 27-42.

[23]. Cryan JF, Mombereau C, Vassout A. The tail suspension test as a model for assessing antidepressant activity: review of pharmacological and genetic studies in mice. Neurosc.Biobehav Rev 2005, 29 (4-5): 571-625.

[24]. Costa-Nunes JP, Cline BH, Araújo-Correia M, Valença A, Markova N, Dolgov O, Kubatiev A, Yeritsyan N, Steinbusch HW, Strekalova T. Animal Models of Depression and Drug Delivery with Food as an Effective Dosing Method: Evidences from Studies with Celecoxib and Dicholine Succinate. Biomed Res Int 2015, 596126.

[25]. Jones MD, Lucki I. Sex differences in the regulation of serotonergic transmission and behaviour in 5-HT receptor knockout mice. Neuropsychopharmacology 2005, 30 (6): 1039-1047.

[26]. Ampuero E, Luarte A, Santibañez M, Varas-Godoy M, Toledo J, DiazVeliz G, Cavada G, Rubio FJ, Wyneken U. Two Chronic Stress Models Based on Movement Restriction in Rats Respond Selectively to Antidepressant 
Drugs: Aldolase C As a Potential Biomarker. Int J Neuropsychopharmacol 2015, pyv038.

[27]. Yoshikawa T, Watanabe A, Ishitsuka Y, Nakaya A, Nakatani N. Identification of multiple genetic loci linked to the propensity for "behavioural despair" in mice. Genome Res 2002, 12 (3): 357-366.

[28]. Guardiola-Lemaître $B$, Lenègre A, Porsolt RD. Combined effects of diazepam and melatonin in two tests for anxiolytic activity in the mouse. Pharmacol Biochem Behav 1992, 41 (2): 405-408.

[29]. Liu X, Gershenfeld HK. Genetic differences in the tail-suspension test and its relationship to imipramine response among 11 inbred strains of mice. Biol Psychiatry 2001, 49 (7): 575-581.

[30]. Malatynska E, Steinbusch HW, Redkozubova O, Bolkunov A, Kubatiev $A$, Yeritsyan NB, et al. Anhedonic-like traits and lack of affective deficits in 18-month-old C57BL/6 mice: Implications for modeling elderly depression. ExpGerontol 2012, 47 (8): 552-564.

[31]. Strekalova T, Evans M, Costa-Nunes J, Bachurin S, Yeritsyan N, Couch Y, Steinbusch HM, Eleonore Köhler S, Lesch KP, Anthony DC. Tlr4 upregulation in the brain accompanies depression- and anxiety-like behaviours induced by a high-cholesterol diet. Brain Behav Immun 2015, 427.

[32]. Porsolt RD, Deniel $M$, Jalfre $M$. Forced swimming in rats: hypothermia, immobility and the effects of imipramine. Eur J Pharmacol 1979, 57 (4): 431-436.

[33]. Bogdanova OV, Kanekar S, D'Anci KE, Renshaw PF. Factors influencing behaviour in the forced swim test. Physiol Behav 2013, 227-39.

[34]. Naudon L1, Jay TM. Opposite behaviours in the forced swimming test are linked to differences in spatial working memory performances in the rat. Neuroscience 2005, 130 (2): 285-293.

[35]. Blanchard RJ, Griebel G, Henrie JA, Blanchard DC. Differentiation of anxiolytic and panicolytic drugs by effects on rat and mouse defence test batteries. Neurosci Biobehav Rev 1997, 21 (6): 783-789.

[36]. Perrault G1, Morel E, Zivkovic B, Sanger DJ. Activity of litoxetine and other serotonin uptake inhibitors in the tail suspension test in mice. Pharmacol Biochem Behav 1992, 42 (1): 45-47. 


\section{Figure legends}

Figure 1. Schematic timeline of tail suspension (A) and forced swim (B) experiments, and (C) tests for anxiety and locomotion.

Figure 2. Effects of $\mathrm{T} 2$ at the dose $0.25 \mathrm{mg} / \mathrm{kg}$ in the tail suspension and the forced swim tests. On Day 1 (A) and Day 2 (B) of the tail suspension test, mice treated with $0.25 \mathrm{mg} / \mathrm{kg}$ of $\mathrm{T} 2(\mathrm{n}=8)$ showed no differences in the latency of immobility and in the duration of this behaviour compared with vehicle-treated control mice $(n=7)$. On Day 1 (C) and Day $2(D)$ of the forced swim test, mice treated with $0.25 \mathrm{mg} / \mathrm{kg}$ of $\mathrm{T} 2(\mathrm{n}=20)$ showed no differences in the latency of floating and in the duration of this behaviour as compared with vehicle-treated control mice $(n=20)$. Mann-Whitney test.

Figure 3. Effects of $\mathrm{T} 2$ at dose $0.75 \mathrm{mg} / \mathrm{kg}$ in the tail suspension and the forced swim tests. (A) In comparison with vehicle-treated control animals ( $\mathrm{n}$ $=5)$, mice treated with $0.75 \mathrm{mg} / \mathrm{kg}$ of $\mathrm{T} 2(\mathrm{n}=8)$ showed no differences in the latency immobility and in the duration of this behaviour on Day 1 of the tail suspension test. (B) The duration of immobility was significantly reduced at first two-minute testing interval of the test on Day 2. In the forced swim test, mice treated with $0.75 \mathrm{mg} / \mathrm{kg}$ of $\mathrm{T} 2(\mathrm{n}=8)$ showed no differences in the latency of floating and in the duration of this behaviour in comparison to control mice $(n=8)$ on Day 1 (C) and Day 2 (D). In the forced swim test, CD1 mice treated with T2 $(n=5)$ showed no differences in the latency of floating and in the duration of this behaviour compared with control mice $(n=5)$ on Day $1(\mathrm{E})$ and Day $2(\mathrm{~F})$. $^{*}-\mathrm{p}<0.05$ versus control; Mann-Whitney test.

Figure 5. Effects of T2 on anxiety and locomotor behaviour. In the dark-light box, $\mathrm{C} 57 \mathrm{BI} / 6$ mice that were treated with T2 at the dose $0.75 \mathrm{mg} / \mathrm{kg}(\mathrm{n}=5)$ did not differ from vehicle-treated mice $(n=6)$ in the latency of first exit $(A)$, time spent (B), and number of exits (C) to the light compartment. In the novel cage (D), C57BI/6 mice that received T2 at the dose $0.25 \mathrm{mg} / \mathrm{kg}(\mathrm{n}=5)$ and CD1 mice treated with $T 2$ at dose $0.75 \mathrm{mg} / \mathrm{kg}(\mathrm{n}=5)$ did not differ from vehicle-treated control $\mathrm{C} 57 \mathrm{BI} / 6$ mice $(n=10)$ and vehicle-treated $C D 1$ mice $(n=6)$, respectively, in the number of rearings. Mann-Whitney test. 


\section{A. Tail suspension test}

$\begin{array}{ccc}-30 \text { min } & \text { Day } 1 & \text { Day } 2 \\ \text { Administration } & \text { Tail suspension } & \text { Tail suspension } \\ \text { of drug or vehicle } & \text { session } & \text { session } \\ & 6 \mathrm{~min} & 6 \mathrm{~min}\end{array}$

B. Forced swim test

$\begin{array}{ccc}-30 \text { min } & \text { Day } 1 & \text { Day } 2 \\ \begin{array}{c}\text { Administration } \\ \text { of drug or vehicle }\end{array} & \text { Swim session } & \text { Swim session } \\ 6 \text { min }\end{array}$

\section{Novel cage and dark-light box}

$\begin{array}{ccc}-30 \mathrm{~min} & 0 \mathrm{~min} & +5 \mathrm{~min} \\ \begin{array}{c}\text { Administration } \\ \text { of drug or vehicle }\end{array} & \text { Dark-light box } & \text { Novel cage } \\ 5 \mathrm{~min} & 5 \mathrm{~min}\end{array}$

Figure 1 
A $0.25 \mathrm{mg} / \mathrm{kg}$ T2

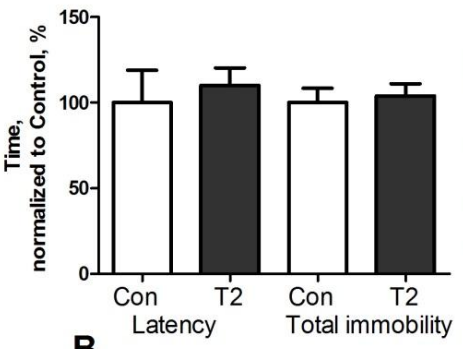

B

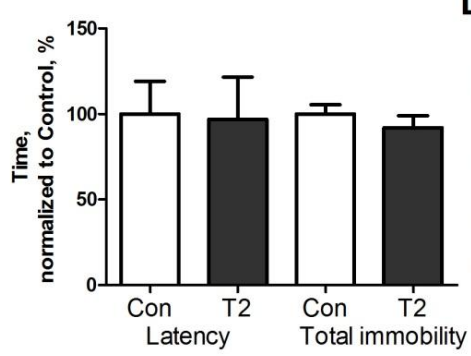

Day 1

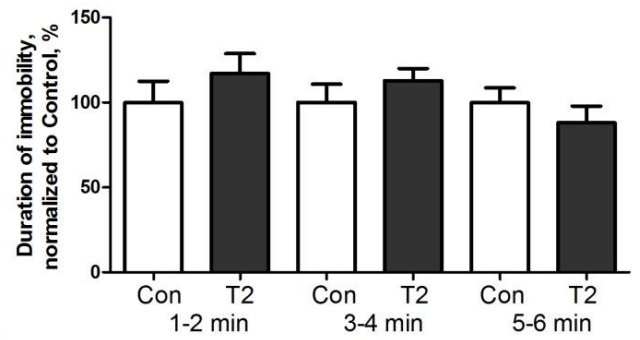

Day 2

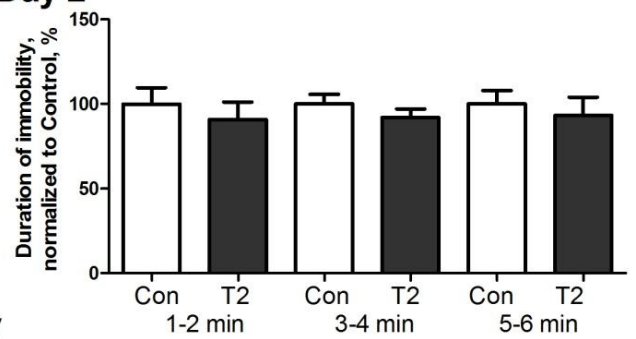

Figure 2

FORCED SWIM TEST

C $\quad 0.25 \mathrm{mg} / \mathrm{kg} \mathrm{T2}$

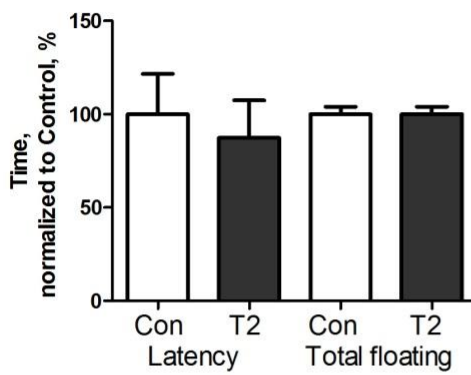

D

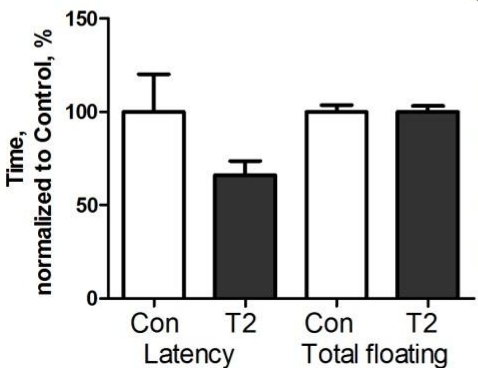

C57BI/6

Day 1

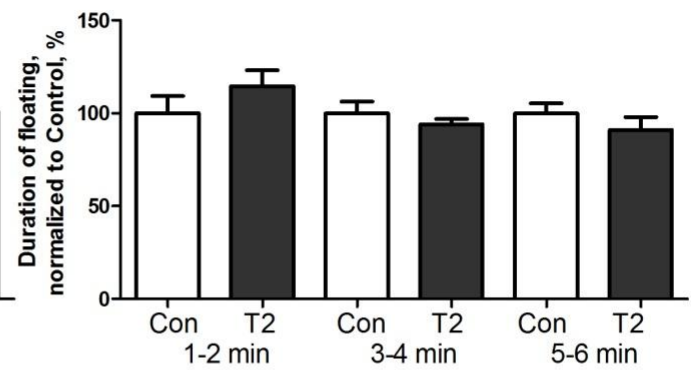

Day 2

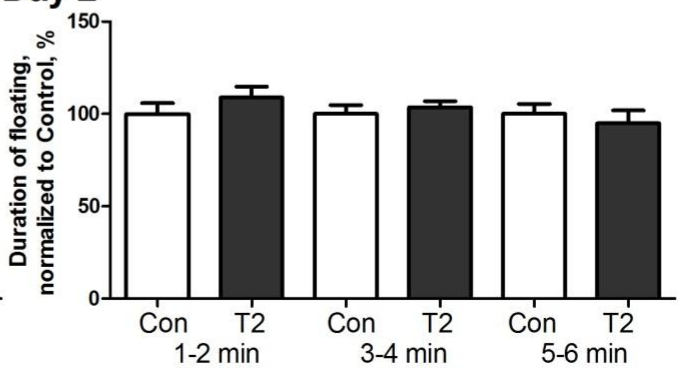




\section{TAIL SUSPENSION TEST C57BI/6 mice}
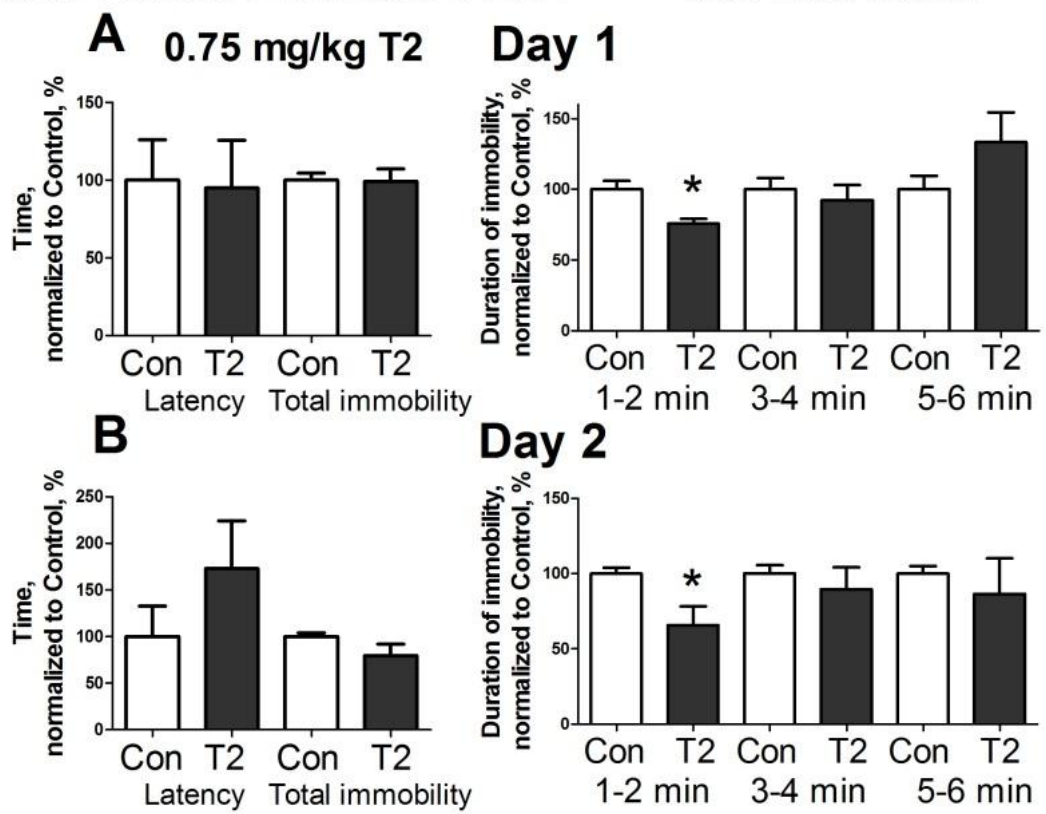

FORCED SWIM TEST

C57BI/6 mice

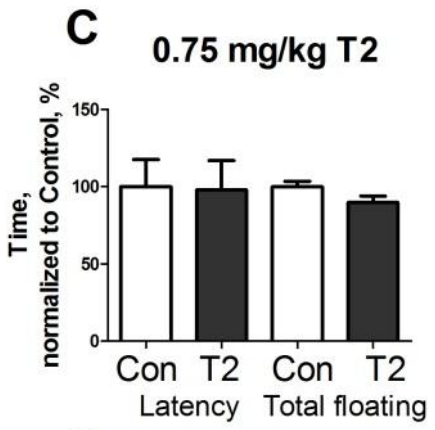

\section{Day 1}

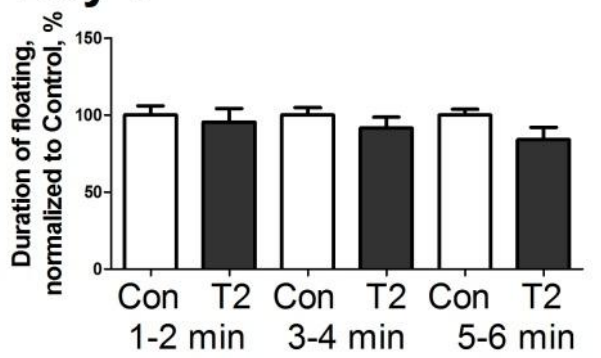

D

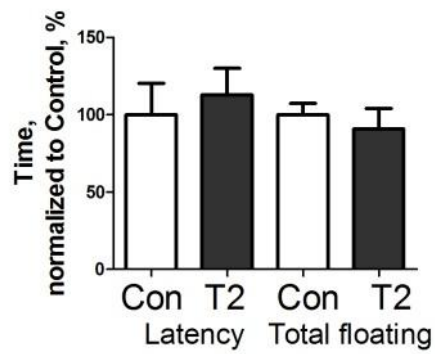

Day 2

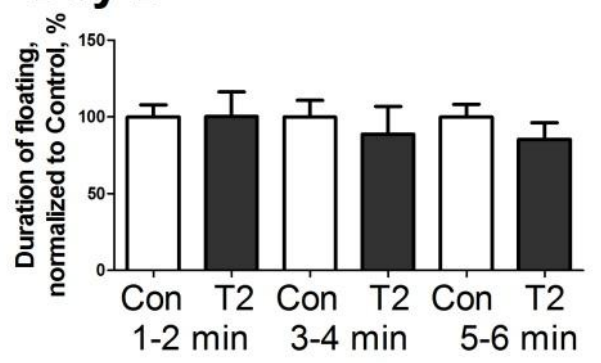




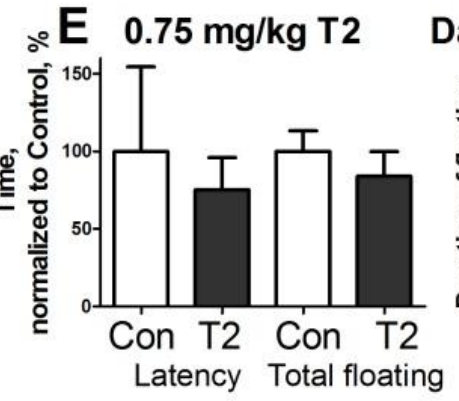

\section{Day. 1}
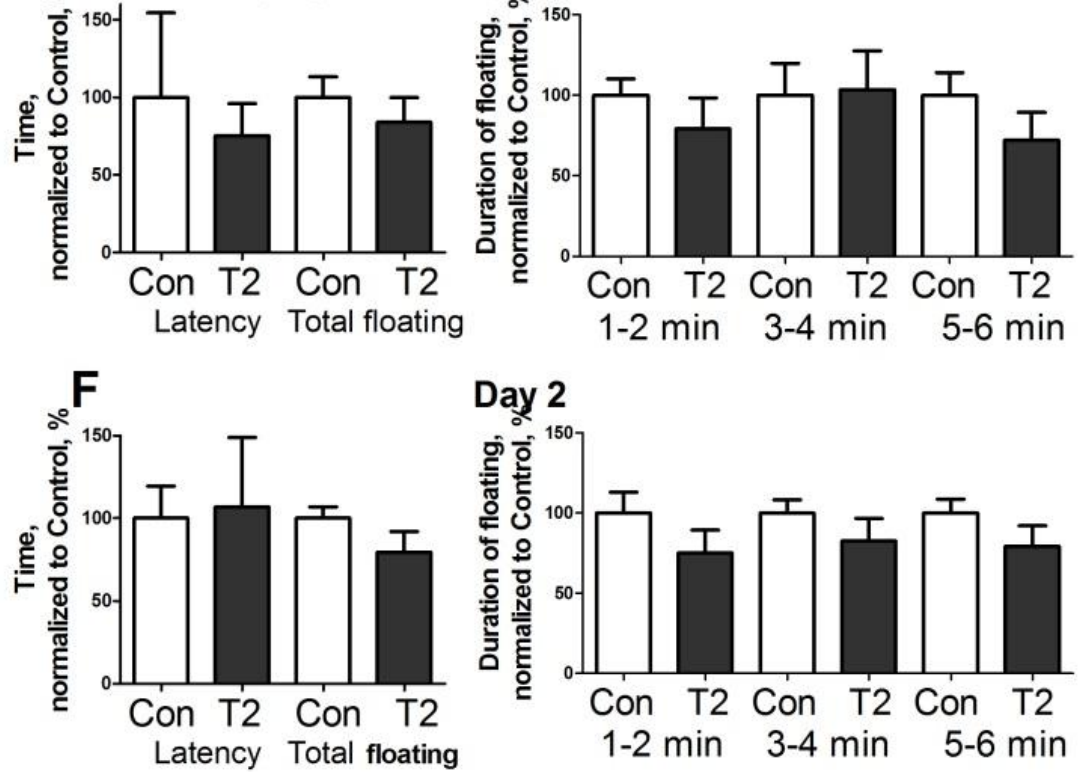

Figure 3 


\section{DARK-LIGHT BOX}
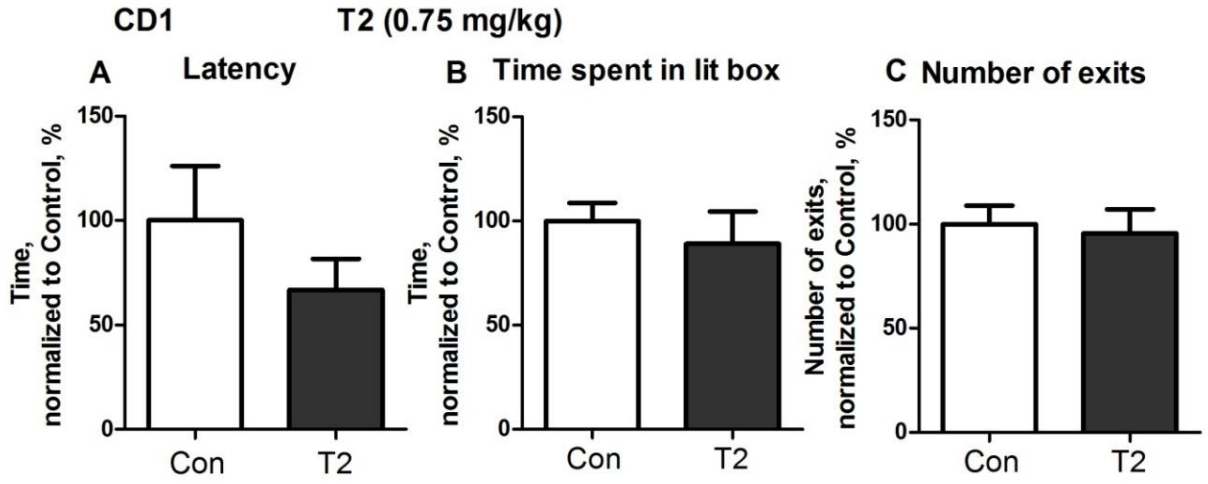

NOVEL CAGE

D
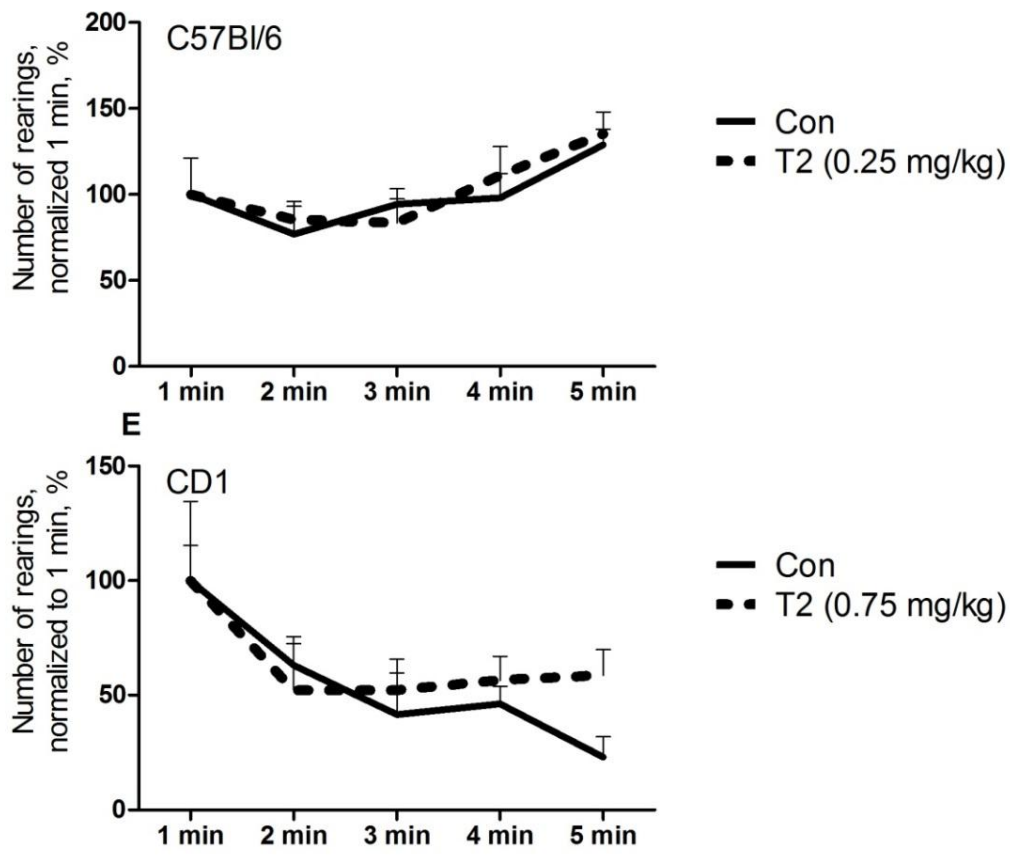

Figure 4 
Chapter 4. Individual differences in behavioural despair predict brain GSK3 $\beta$ expression in mice: the power of a modified swim test Nataliia Markova ${ }^{1,2,3+}$, Tatyana Strekalova ${ }^{1+*}$, Elena Shevtsova ${ }^{2}$, Olga Zubareva $^{4}$, Anastassia Bachmet ${ }^{5}$, Harry M Steinbusch ${ }^{1}$, Sergey Bachurin ${ }^{2}$, and Klaus-Peter Lesch $^{1,6^{*}}$

${ }^{1}$ Department of Neuroscience, School for Mental Health and Neuroscience, Maastricht University, Universiteitssingel 40, NL 6229 ER Maastricht, The Netherlands; ${ }^{2}$ Department of Biomolecular Screening, Institute of Physiologically Active Compounds, Russian Academy of Sciences, Severnii proezd, 1, 142432, Chernogolovka, Russia; ${ }^{3}$ Department of Cognitive Dysfunction, Institute of General Pathology and Pathophysiology, Baltiiskaya str, 8, 125315, Moscow, Russia; ${ }^{4}$ Department of Physiology, Federal State Budgetary Scientific Institution "Institute of Experimental Medicine", Akademika Pavlova str, 12, 197022, Saint-Petersburg, Russia; ${ }^{5}$ Department of Human Anatomy, I.M. Sechenov First Moscow State Medical University, Mokhovaya str., 10-3, 119991, Moscow, Russia; ${ }^{6}$ Division of Molecular Psychiatry, Laboratory of Translational Neuroscience, Department of Psychiatry, Psychosomatics and Psychotherapy, University of Wuerzburg, Fuechsleinstrasse 15, 97080, Wuerzburg, Germany

+ equal contribution

*For correspondence:

Prof. Klaus-Peter Lesch, E-mail: kplesch@mail.uni-wuerzburg.de

Dr. Tatyana Strekalova, M.D., E-mail: t.strekalova@maastrichtuniversity.nl

Markova, N., Strekalova, T., Shevtsova, E., Zubareva, O., Bakhmet, A., Steinbusch, H.M., Bachurin, S., Lesch, K.-P., 2016. Individual Differences in Behavioural Despair Predict Brain GSK36 Expression in Mice: The Power of a Modified Swim Test. Neural Plast. 2016, 5098591. 


\section{Abstract}

While deficient brain plasticity is a well-established pathophysiologic feature of depression, little is known about disorder-associated enhanced cognitive processing. Here, we studied a novel mouse paradigm that potentially models augmented learning of adverse memories during development of a depressive-like state. We used a modification of the classic two-day protocol of a mouse Porsolt test with an additional session occurring on Day 5 following the initial exposure. Unexpectedly, floating behaviour and brain glycogen synthase kinase-3 beta (GSK3 $\beta$ ) mRNA levels, a factor of synaptic plasticity as well as a marker of distress and depression, were increased during the additional swimming session that was prevented by imipramine. Observed increases of GSK3 $\beta$ mRNA in prefrontal cortex during delayed testing session correlated with individual parameters of behavioural despair that was not found in the classic Porsolt test. Repeated swim exposure was accompanied by a lower pGSK3 $\beta / G S K 3 \beta$ ratio. A replacement of the second or the final swim sessions with exposure to the context of testing resulted in increased GSK3 $\beta$ mRNA level similar to the effects of swimming, while exclusion of the second testing prevented these changes. Together, our findings implicate the activation of brain GSK3 $\beta$ expression in enhanced contextual conditioning of adverse memories, which is associated with an individual susceptibility to a depressive syndrome.

Keywords: Depression, contextual conditioning, glycogen synthase kinase-3 beta (GSK3 $\beta$ ), hippocampus, prefrontal cortex, animal model, Porsolt's swim test, mice.

\section{Introduction}

One of the most critical elements in the pathophysiology of severe clinical depression might entail augmented cognitive processing of adverse events that can aggravate deleterious effects of primarily negative experiences and precipitate the development of stress-related depressive syndromes [1-3]. Currently available data show that enhanced acquisition of fear memories and adverse experiences can be a relevant pathophysiologic mechanism in 
the development of neuropsychiatric conditions, such as post-traumatic stress disorder, generalized anxiety disorder, phobias, and depression [4, 5]. Nevertheless, the mechanisms underlying reinforced learning of adverse events associated with experience of a specific context during the development of a depressive-like state are poorly understood [6]. The identification of the neurobiology linked to enhanced cognitive processing of aversive experiences would be highly valuable for finding novel therapeutic targets for the treatment of diseases associated with inappropriate retention and processing of memories for environmental adversity.

Animals and humans develop helplessness and fear of environmental contexts in which they have repeatedly experienced aversive events. Most of currently available rodent models of these conditions employ paradigms in which animals learn to associate an aversive unconditioned stimulus, such as a brief foot shock, with a neutral conditioned stimulus, typically a context or a tone. In these models, slow extinction learning is usually taken as a measure of inappropriate retention of fear memories and a posttraumatic stress state [7, 8]. While animal models of enhanced learning of adverse challenges including the above-mentioned type of paradigms are wellestablished for phobias, generalized anxiety, and post-traumatic stress disorders, few data are available concerning modeling of cognitive processing of adversities in the development of a depressive syndrome.

Short tests for depressive-like behaviour, such as the Porsolt swim and tail suspension tests, are commonly used in translational research on depression [9-11]. A classical protocol of Porsolt's test is based on the repeated exposure of rodents to an inescapable short swim, an adverse procedure that is typically repeated in the same test condition with a $24 \mathrm{~h}$ time interval, when animals display an increase of floating behaviour, commonly referred to as "behavioural despair" [12]. A re-exposure of an animal to the originally presented adverse situation is intended to produce a state of (learned) helplessness, one of behavioural hallmarks of depression [12, 13]. Porsolt's test was shown to implicate elements of contextual learning; pharmacologic inhibitors of memory formation were found to attenuate the potentiation of floating during repeated swim sessions [14]. The alleviation 
of floating in this test by antidepressant drugs was shown to be partly mediated by their diminishing effects on contextual learning $[14,15]$.

Evidence suggests that with the recollection of stressful and traumatic experiences resulting in the development of depressive-like features, a phase of memory consolidation might be of particular relevance $[16,17]$. Based on this, we tested the hypothesis that modification of the classic twosession protocol of Porsolt's test in the mouse, with an additional session occurring on Day 5 following the initial exposure, may potentially model the initiation of consolidation processes that are relevant to the development of a depressive-like state and allow to elucidate their neurobiologic mechanisms experimentally. The timing of the employed protocol of modified swim test was based on available data concerning a time curve of a consolidation of contextual fear memories in respective rodent paradigms, where the primary neuronal processes of a fear consolidation last about $48 \mathrm{~h}$ after conditioning and predominantly implicate the hippocampus and prefrontal cortex $[18,19]$. We hypothesized that a similar time window might be critical for a formation of a "depressive-like" neurobiological trace related to enhanced cognitive processing of adverse experiences in a modified swim test protocol proposed here. While repeated forced swimming is often applied to induce more pronounced depressive-like phenotypes and helpless behaviour $[1,20,21]$, there are no reports available that describe the application of this test for modeling enhanced cognitive processing of adverse experiences.

The aim of the current work was to address the question whether a modified swim test with additional delayed session on Day 5 may model increased contextual consolidation of environmental adversities related to the testing. Therefore, we sought to study whether floating behaviour will be further increased during the delayed swimming on Day 5 . In addition, we investigated whether glycogen synthase kinase-3 beta (GSK3 $\beta$ ) expression is altered in limbic structures during modified swim test protocol. GSK3 $\beta$ is a marker of synaptic plasticity in the hippocampus but also of distress and depression. The antibipolar drug lithium and selective GSK-3 inhibitors were reported to increase of GSK3 $\beta$ phosphorylation at serine 9 position, thus, 
decreasing its activity that was associated with beneficial neurobiological and therapeutic effects of these compounds [22-26]. GSK3 $\beta$-dependent functional changes in the hippocampus and prefrontal cortex may influence both anxiety- and depressive-like behaviour, including floating in the forced swim test and cognitive processes [25, 27-33]. Both structures are involved in the consolidation of contextual fear, long-term memories, and enhanced learning during post-traumatic stress disorder [34-37], as well as in the mechanisms of depression [30, 38, 39].

Since we found an increase in both floating behaviour and mRNA GSK3 $\beta$ and correlation between them, in the modified swim test, we further investigated the role of exposures of mice to the context of testing versus exposure to the swim test and omission of testing, in the development of above-mentioned molecular and behavioural effects induced in the modified swim paradigm. Also, we addressed the role of timing by carrying out a "premature" testing on Day 3 following the initial swimming instead of a session on Day 5. In addition, we determined the levels of phosphorylated and total GSK3 $\beta$, as well as their ratio, in the hippocampus and prefrontal cortex of mice exposed to the modified swim test. Finally, these behavioural and molecular read-outs were investigated after a fourteen-day treatment with a low dose of the antidepressant imipramine.

Our studies demonstrated the role of contextual elements and timing with adverse experiences of inescapable swimming, in the induction of behavioural and molecular alterations associated with a depressive-like state. They also implicate upregulation of GSK3 $\beta$ in the mechanism of enhanced floating response in individual mice that are predisposed to behaviour despair in this modified swim paradigm. This led us to speculate that GSK3 $\beta$, which is both a critical mediator in processes associated with synaptic plasticity and a marker of distress and maladaptation, may play an integrative role in mediating pathologically enhanced memory consolidation for noxious stimulation and a development of associated depressive features. 


\section{Materials and methods}

\section{Animals}

Studies were performed using 3-month-old male C57BL/6J from RAS, Moscow region, a provider licensed by Charles River (http://www.spfanimals.ru/about/providers/animals/). Mice were single-housed under a reversed 12-hour light-dark cycle (lights on: 21:00 h) starting from the day of animals' transportation in the laboratory with food and water provided ad libitum, under controllable laboratory conditions (22 $\pm 1^{\circ} \mathrm{C}, 55 \%$ humidity). All experiments were carried out in accordance with the European Communities Council Directive for the care and use of laboratory animals 2010/63/EU and were approved locally (IPAC NM3401165).

\section{Experimental design}

In the first study we investigated whether in the additional delayed testing in the Porsolt swim test mice display an increase or a decrease of behavioural despair as compared to the classic swim test. Therefore, mice were subjected to the one- and two-session protocols of Porsolt's test, as well as to the modified protocol during which, on Day 5 after initial testing on Day 1 , a delayed session was added to a two-day swim test (Figure 1(a)). Their floating behaviour was evaluated in 6-min sessions in 2-min time intervals (see below). The testing was carried out in morning hours, during active period of animal's cycle, in dark laboratory rooms where the apparatuses were lit with subtle lighting (see below). For acclimatization, mice were transported to the experimental room from the lab where they were housed, at least $1 \mathrm{~h}$ prior the start of testing. Behaviour was video-recorded and scored offline.

As an increase of floating behaviour on Day 5 was found in above-described experiment, we predicted that this augmentation in behavioural despair is due to cognitive processes taking place during the delay between swim sessions. In the next series of experiments (Figures $1(b)-1(f)$ ) we therefore addressed the individual role of context, swimming, and timing of testing in 
this phenomenon. Therefore, a replacement of a swim session with an exposure to the context of testing or complete omission of any behavioural manipulation was performed on Day 2 (Figures $1(b)$ and $1(c)$ ) and on Day 5 (Figures $1(\mathrm{~d})$ and $1(\mathrm{e})$ ). Data were calculated in percent from mean values of floating on Day 1 for each group. Additionally, a group of mice was tested "prematurely" in a three-session swim test where the last test was carried out on Day 3 instead of Day 5 (Figure 1(f)) and compared with a group subjected to the regular protocol of the modified swim test (Figure 1(a)). A study design for this and described below series of experiments is additionally summarized in a Supplementary Tables in Supplementary Material available online at http://dx.doi.org/10.1155/2016/5098591.

In all above-described schemes of testing, mice were sacrificed $10 \mathrm{~min}$ after the last behavioural procedure and two brain regions, hippocampus and prefrontal cortex, were dissected for subsequent analyses of GSK3 $\beta$ activities using qRT-PCR and ELISA of total and phosphorylated forms of GSK3 $\beta$ (see below). While defining a time frame of analysis of GSK3 $\beta$ activities, the changes in brain GSK3 $\beta$ mRNA at Day 5 , the primary experimental setting of interest, were found at the time point $10 \mathrm{~min}$, but not $24 \mathrm{~h}$ after testing. Based on these data, and in sake of conformity in the assessments of gene expression and protein changes of GSK3 $\beta$, we compared their measures between all applied experimental schemes of swim test at time point $10 \mathrm{~min}$ after the last swim session, unless specified. A group of intact control mice that were naïve for swim experience was used in these assays; each study employed a separate control group. Spearman correlation was performed to link changes in GSK3 $\beta$ mRNA concentration to the duration of floating. Additionally, mice were assigned to subgroups designated "high-" and "lowfloaters" according to a criterion of "mean of total duration of floating of the group" as that was measured in the swim session preceding a sacrifice of the animal, and the expression of GSK3 $\beta$ was compared. Mice which displayed a total duration of floating exceeding the mean of the group at the last testing session were assigned to a subgroup of "high-floaters." Remaining animals were considered as "low-floaters"; their values of total floating duration measured at the last swim session were below the mean of the group. 
Also, in the regular protocol of modified swim test (Figure 1(a)), a subgroup of mice was sacrificed $24 \mathrm{~h}$ after the last swimming and compared for GSK3 $\beta$ expression with a group sacrificed $10 \mathrm{~min}$ thereafter. In addition, in the regular protocol of modified swim test, qRT-PCR of GSK-3alpha was carried out to control for the specificity of changes in GSK3 $\beta$ expression.

In the final study, we compared floating behaviour and GSK3 $\beta$ mRNA/protein levels in naïve mice and mice pretreated for fourteen days with a low dose of $7.5 \mathrm{mg} / \mathrm{kg} / \mathrm{day}$ of the antidepressant imipramine (Figure $1(\mathrm{~g})$; see below). The selection of the imipramine dosage was based on previous findings showing absence of confounding effects of this dose on locomotion and weak antidepressant effects in the classic Porsolt swim test in C57BL6 mice [40].

Classic and modified swim tests with additional delayed testing

Mice were subjected to one or two swimming sessions with an interval of 24 h (classic protocols of swim test), or three swim sessions, where the third test was added to a two-day "classic protocol" and carried out on Day 5 following the initial test on Day 1 (modified swim test; Fig. 1A). All sessions were 6-min long and were performed by placing a mouse in a transparent cylinder $(\varnothing 17 \mathrm{~cm})$ filled with water $\left(+23{ }^{\circ} \mathrm{C}\right.$, water height $13 \mathrm{~cm}$, height of cylinder $20 \mathrm{~cm}$ ). The duration of floating behaviour that was defined by the absence of any directed movements of the animals' head and body, was scored manually using criteria, which were previously validated by automated scoring with Noldus EthoVision XT 8.5 (Noldus Information Technology, Wageningen, The Netherlands) and CleverSys (CleverSys, Reston, VA, USA) as described elsewhere [41, 42]. Time spent with floating was evaluated at 2-min intervals as well as summarized for the entire duration of the test. In the experiments with various combinations of swim test protocols, exposures to a context of testing and timing of sacrifice, as well as imipramine dosing, the total duration of floating was evaluated for the entire 6-min periods. In all studies, except one, which addressed the GSK3 $\beta$ expression $24 \mathrm{~h}$ post-swimming in the modified swim test, mice were sacrificed ten minutes after swimming (see below). 


\section{Exposure to context of swimming}

To address the role of context in behavioural and molecular changes found in the modified swim test, a series of experiments in which mice were subjected to the context of a swimming test at various time points of testing. Therefore, in the same environment where they were previously subjected to swimming sessions, mice were taken out from the cage by an experimenter and exposed to the swimming pool being hung by their tails and kept above a water surface for $1 \mathrm{~s}$. Thereafter, they were placed back to their home cages. We chose to apply short-lasting reminding procedure of above-described protocol in order to prevent potential behavioural and molecular aberrations associated with a prolonged suspension of mice by their tails. Based on our previous findings that handling, as an element of this procedure, might be a powerful contextual cue for mice, a sufficient efficacy of selected protocol of reminding procedure was anticipated [43].

\section{Brain dissection, RNA extraction and $q R T-P C R$}

Mice were sacrificed by cervical dislocation as described elsewhere [42] in the experimental area where swim test was performed, except that specific studies were mice supposed to be not exposed to the context of testing prior to sacrifice. The brain of each mouse was dissected and the prefrontal cortex and hippocampus were isolated as previously reported [44-46] and stored at $-80^{\circ} \mathrm{C}$ until use. mRNA was extracted by using TRI Reagent (MRC, Cincinnati, OH, USA). First-strand cDNA synthesis was performed using random primers and Superscript III transcriptase (Invitrogen, Darmstadt, Germany); 1 mcg total RNA was converted into cDNA. Quantitative RT-PCR (qRT-PCR) of the GSK3 $\beta$ gene and the housekeeping gene glyceraldehyde-3phosphate dehydrogenase (GAPDH) was performed using the SYBR Green master mix (Bio-Rad Labouratories, Philadelphia, PA, USA) and the CFX96 Real-time System (Bio-Rad Labouratories, Philadelphia, PA, USA). The reference gene was selected in preliminary experiments that demonstrated relatively low variability in GAPDH expression in the limbic structures of the rodents exposed to various stressors, including forced swimming, as compared to the expression of several other housekeeping genes that is in line with other reports [47-49]. Sequences of primers used are listed in 
Supplementary Material (Supplementary Table 1). Data were normalized to GAPDH mRNA expression and calculated as relative-fold changes compared to control mice as described elsewhere [44].

Results of qRT-PCR measurement were expressed as $\mathrm{Ct}$ values, where $\mathrm{Ct}$ is defined as the threshold cycle of PCR at which amplified product was $0.05 \%$ of normalized maximal signal. We used the comparative $\mathrm{Ct}$ method and computed the difference between the expression of the gene of interest and GAPDH expression in each cDNA sample (2- $\Delta \Delta C \mathrm{Ct}$ method). Data are given as expression-fold compared to the mean expression values in nonstressed control mice [44].

\section{ELISA assays of total and phosphorylated forms of GSK3B}

Mice were sacrificed by cervical dislocation as described elsewhere [42] in the experimental area where swim test was performed, except that specific studies were mice supposed to be not exposed to the context of testing prior to sacrifice. The brain of each mouse was dissected and the prefrontal cortex and hippocampus were isolated as previously reported [44-46] and stored at $-80^{\circ} \mathrm{C}$ until use. mRNA was extracted by using TRI Reagent (MRC, Cincinnati, OH, USA). First-strand cDNA synthesis was performed using random primers and Superscript III transcriptase (Invitrogen, Darmstadt, Germany); 1 mcg total RNA was converted into cDNA. Quantitative RT-PCR (qRT-PCR) of the GSK3 $\beta$ gene and the housekeeping gene glyceraldehyde-3phosphate dehydrogenase (GAPDH) was performed using the SYBR Green master mix (Bio-Rad Labouratories, Philadelphia, PA, USA) and the CFX96 Real-time System (Bio-Rad Labouratories, Philadelphia, PA, USA). The reference gene was selected in preliminary experiments that demonstrated relatively low variability in GAPDH expression in the limbic structures of the rodents exposed to various stressors, including forced swimming, as compared to the expression of several other housekeeping genes that is in line with other reports [47-49]. Sequences of primers used are listed in Supplementary Material (Supplementary Table 1). Data were normalized to GAPDH mRNA expression and calculated as relative-fold changes compared to control mice as described elsewhere [44]. 
Results of qRT-PCR measurement were expressed as Ct values, where $\mathrm{Ct}$ is defined as the threshold cycle of PCR at which amplified product was $0.05 \%$ of normalized maximal signal. We used the comparative $\mathrm{Ct}$ method and computed the difference between the expression of the gene of interest and GAPDH expression in each cDNA sample (2- $\Delta \Delta \mathrm{Ct}$ method). Data are given as expression-fold compared to the mean expression values in nonstressed control mice [44].

\section{Drug administration}

Imipramine (Sigma-Aldrich, St. Louis, MO, USA) was dissolved in tap water; the dose of drug was $7.5 \mathrm{mg} / \mathrm{kg}$ and provided for mice to drink ad libitum in water bottles for fourteen days prior the beginning of behavioural testing and throughout the entire experiment as described elsewhere $[45,51]$. The method of dosing was selected in order to exclude a factor of handling that could be a significant confound of daily intraperitoneal injections or administration of a drug via a gavage with the current model.

\section{Statistical analysis}

Data were analyzed with GraphPad Prism version 5.0 for Windows (San Diego, CA, USA). Paired two-tailed test was used to compare repeated measurements. One-way ANOVA was used to compare three or more groups and two-way ANOVA was applied to analyze "high-" and "low-floaters"; Tukey's post hoc multiple comparison test was employed as a post hoc test. The trend of the effects of a number of swim sessions on ELISA parameters was analyzed using univariate ANOVA for three groups and post hoc test for linear trend. Spearman correlation was used to perform correlational analysis. The level of confidence was set at $95 \%(p<0.05)$ and data are shown as mean \pm SEM.

\section{Results}

Potentiated behavioural despair in the modified swim paradigm

Repeated measures ANOVA showed significant differences between sessions on Day 1 and Day 2 and Day 2 and Day 5 ( $F=25.16 ; p<0.0001$, Repeated 
measures ANOVA summary; Figure 2(a)). While during delayed testing in the modified swim protocol mice could potentially display decreased duration of floating due, for instance, fading away of adverse effect of previous inescapable swimming, repeated pairwise comparison revealed a significant increase of the total duration of floating from Day 2 to Day $3(q=4.85 ; p=$ 0.012 , Tukey's test; Figure 2(a)). There was a significant increase of this parameter from Day 1 to Day 2 as well ( $q=5.01 ; p=0.009$, Tukey's test), confirming generally reported data [31]. The increase of despair behaviour at the delayed testing session on Day 5 occurred at the expense of the first two minutes of the test where the duration of floating was sharply elevated as compared to this observation period of the session on Day $2(q=5.01 ; p=$ 0.009 , Tukey's test; Figures 2(b) and 2(c)). No further enhancement of floating behaviour was found during the second two-minute testing interval and the last two minutes of the swim session as compared to these observation periods on Day $2(q=3.26 ; p=0.091$ and $q=3.32 ; p=0.085$, resp., Tukey's test; Figure 2(d)). An instantaneous increase of floating during testing at the postponed session instead of a gradual increase throughout the testing procedure suggests that the animals were likely to be in a state predisposing to potentiated floating behaviour and displayed this immediately at placement in a pool.

The role of context, swimming and timing of testing in the potentiation of floating behaviour during the modified swim test

Next, in a series of experiments, we addressed the impact of context, swimming, and timing of testing, in the potentiation of floating behaviour during the modified swim test (see Supplementary Table 2). A comparison of groups that were subjected to modified swim test (Figure 1(a)) or similar protocols where the swimming session on Day 2 was replaced with exposure to a context of testing (Figure $1(\mathrm{~d})$ ) or omitted (Figure $1(\mathrm{c})$ ) revealed significant differences in the floating duration on Day $5(F=4.79 ; p=0.017$, ANOVA; Figure 2(e)). Mice from the latter group, but not mice exposed to the context of testing on Day 2, showed a significant decrease in this parameter, as compared with mice exposed to the modified swim test ( $q=$ 4.31; $p=0.014$ and $q=1.56 ; p=0.521$, resp., Tukey's test; Figure 2(e)). This 
suggests that exposure to a swim at on Day 2 or a context of testing are crucial for the potentiation of floating on Day 5 and that both have similar impact in this potentiation.

In the next experiment that studied whether enhancement of floating duration during additional session may occur already on Day 3 (Figure 1(f)), repeated measures ANOVA showed significant differences between the days of testing ( $F=15.52 ; p=0.0005$, repeated measures ANOVA; Figure 2(f)). Pairwise comparison showed that, in comparison to the session on Day 1 , the duration of floating in these mice was significantly higher on Day 2 and Day 3, but no difference was found between two latter sessions $(q=7.06 ; p$ $=0.002, q=6.23 ; p=0.004$, and $q=2.20 ; p=0.312$, resp., Tukey's test; Figure $2(\mathrm{f})$ ). This suggests that time interval between repeated sessions is a factor of potentiation of floating behaviour in the modified swim test.

The role of context, swimming and timing of testing in the altered GSK3B brain expression during the modified swim test

A comparison of GSK3 $\beta$ mRNA levels in the hippocampus of intact mice and groups of mice sacrificed on Day 1, Day 2, or on Day 5 (Figure 1(a); see Suppl. Table 3) revealed significant group differences $(F=9.77 ; p<0.0001$, ANOVA; Figure $3(a)$ ). In comparison to intact control group, this measure was significantly elevated only on Day 5 of testing $(q=3.977 ; p=0.0355$, Tukey's test; Figure 3(a)), but not on Day 1 and on Day 2 ( $q=2.465 ; p=0.3154$ and $q$ $=2.238 ; p=0.3988$, resp.; Figure 3(a)). Mice subjected to modified swim test had significantly higher hippocampal expression of GSK3 $\beta$ than two latter groups ( $q=0.1753 ; p=0.0001$ and $q=6.3464 ; p=0.0003$, resp.; Figure 3(a)).

ANOVA revealed no significant group differences in GSK3 $\beta$ mRNA levels in the prefrontal cortex $(F=0.7102 ; p=0.5523$, ANOVA; Figure $3(b))$. In comparison to the intact control group, this parameter was not significantly altered on Day 1, Day 2, and Day 5 ( $q=0.9227 ; p=0.9140, q=0.5839 ; p=$ 0.9758 , and $q=0.9656 ; p=0.9030$, resp., Tukey's test; Figure 3(b)). Mice subjected to modified swim test had no differences in the expression of GSK3 $\beta$ in this brain structure as compared to the two latter groups $(q=$ $1.881 ; p=0.5505$ and $q=1.657 ; p=0.6484$, resp.). 
In a separate experiment, we compared GSK3 $\beta$ mRNA levels in the brain of mice from the modified forced swim test experiment that were sacrificed either $10 \mathrm{~min}$ or $24 \mathrm{~h}$ after the last swimming session. ANOVA revealed group differences in this measure in hippocampus but not in prefrontal cortex $(F=6.170 ; p=0.0103$ and $F=0.8936 ; p=0.4276$, resp., ANOVA; Figures $3(\mathrm{c})$ and $3(\mathrm{~d})$ ). Post hoc Tukey's test showed a significant increase in GSK3 $\beta$ mRNA levels in the hippocampus of mice sacrificed 10 min but not $24 \mathrm{~h}$ after test, as compared to intact group $(\mathrm{q}=4.459 ; \mathrm{p}=0.0160$ and $\mathrm{q}=$ $0.1155 ; p=0.9963$, resp.; Figure 3(c)). No group differences in GSK3 $\beta$ mRNA levels in the prefrontal cortex was found, post hoc analysis showed a lack of changes for either group tested in the swim test, in comparison with intact group $(q=1.821 ; p=0.4209$ and $q=0.4293 ; p=0.9506$, resp., Tukey test; Figure $3(d))$. These data independently replicate the findings with changes of GSK3 $\beta$ expression during modified forced swim test presented herein and define selected time point +10 min after testing as the likely optimal time period for assessment of this measure in behavioural protocols.

A comparison of GSK3 $\beta$ mRNA levels of intact mice and of mice subjected to the context of testing instead of testing itself on Day 5 (Figure 1(d)), animals that were not exposed to either swimming or context and sacrificed on Day 5 (Figure 1(e)) and mice tested in "premature" last swim on Day 3 (Figure $1(f)$ ), did not reveal significant group differences for the hippocampus $(F=$ $0.2499 ; p=0.8605$, ANOVA; Figure 3(e)), but for the prefrontal cortex $(F=$ 3.907; $p=0.0231$, ANOVA; Figure 3(d)). As compared to the intact control, GSK3 $\beta$ mRNA was significantly elevated in prefrontal cortex of mice subjected to the modified swim test in which swimming Day 5 was replaced with their exposure to a context of testing $(q=3.945 ; p=0.0497$, Tukey's test; Figure 3(f)); no other group differences were found in this measure. There were no significant changes in the hippocampal GSK3 $\beta$ mRNA between these groups $(q=0.7750 ; p=0.9461$; Figure 3(e)). These data provide evidence for a role of timing and exposure to the context of testing in increased GSK3 $\beta$ expression during repeated exposure to a swim test.

To control for the specificity of changes in GSK3 $\beta$ expression in mice subjected to the modified swim test, the expression of brain GSK-3alpha 
mRNA was assessed on Day 5 in the same experiment (Figure 1(a)). We found no differences in this measure in both hippocampus and the prefrontal cortex between intact mice and mice subjected to the modified swim test $(p=0.7833$ and $p=0.1852$, resp., t-test; Figures $3(g)$ and $3(h))$, suggesting a specificity of the above-described alternations of GSK3 $\beta$ mRNA levels in these brain structures.

Changes of brain total and phosphorylated GSK3B during modified swim test

ANOVA showed that there were no significant differences in brain total GSK3 $\beta$ in mice on Day 1, Day 2, and Day 5 of swim testing in the hippocampus, but in the prefrontal cortex $(F=1.958 ; p=0.1363$ and $F=$ 3.016; $p=0.0420$, ANOVA; Figures 4(a) and 4(b); see Supplementary Table $3)$. In the prefrontal cortex, brain total GSK3 $\beta$ was significantly higher on Day 1 , but not on Day 2 and Day 5 of the swim test, where a non-significant increase of this measure was found in comparison with intact control $(q=$ 4.144; $p=0.0283, q=2.089 ; p=0.4611$, and $q=2.999 ; p=0.1655$, Tukey's test; Figures $4(a)$ and $4(b))$.

There were significant group differences in the levels of pS9-GSK3 $\beta$ on Day 1 , Day 2, and Day 5 of swim testing in the hippocampus and in the prefrontal cortex $(F=13.04 ; p<0.0001$ and $F=12.11 ; p<0.0001$, resp., ANOVA; Figures $4(c)$ and $4(d))$. Post hoc analysis revealed a significant decrease of this measure in both of these brain areas only on Day 2 of swim test ( $q=$ 6.917; $p=0.0019$ and $q=2.950 ; p<0.0001$, resp., Tukey's test; Figures 4 (c) and $4(d))$ and Day $5(q=6.618 ; p=0.0002$ and $q=6.868 ; p=0.0001$, resp.; Figures $4(c)$ and $4(d))$, suggesting that repeated but not a single testing reduces the levels of inactive form of GSK3 $\beta$.

Similarly, there were significant group differences in the ratio of levels of pGSK3 $\beta /$ total GSK3 $\beta$ between groups of mice sacrificed on Day 1, Day 2, and Day 5 of swim testing, both in the hippocampus and in the prefrontal cortex $(F=11.74 ; p<0.0001$ and $F=8.156 ; p=0.0003$, ANOVA; Figures 4(e) and $4(f))$. Post hoc analysis revealed a significant decrease of this ratio in both of these brain areas on Day $2(q=5.111 ; p=0.0048$ and $q=4.449 ; p=0.0172$, Tukey's test; Figures 4(e) and 4(f)) and Day $5(q=7.410 ; p<0.0001$ and $q=$ 
5.662; $p=0.0017$; Figures 4(e) and 4(f)). Moreover, the trend of the effects of a number of swim sessions on this parameter was found using univariate ANOVA for three groups and post hoc test for linear trend (hippocampus: slope $-0.3301, \mathrm{R} 2=0.5180 ; \mathrm{p}<0.0001$ and prefrontal cortex: slope -0.2277 , $R 2=0.4006 ; p<0.0001$, Posttest for linear trend; Figures 4(e) and 4(f)).

Differential activities of GSK3B in mice with low and high floating in the modified swim test

Mice were assigned to groups of "high-" and "low-floaters" based on the mean value of total duration of floating in the swim session preceding a sacrifice and compared for brain GSK3 $\beta$ activities (see Supplementary Table 4). Two-way ANOVA revealed no significant differences between these subgroups studied for GSK3 $\beta$ mRNA levels on Day 1, Day 2, and Day 5 both in the hippocampus and prefrontal cortex $(F=0.1973 ; p=0.6588$ and $F=$ $0.3826 ; p=0.5395$, resp., two-way ANOVA; Figures 5(a) and 5(b)). Both highfloaters and low-floaters displayed significant increase of this measure in the hippocampus after swim session on Day 5 as compared to the session on Day $2(q=3.787 ; p=0.0478$ and $q=4.690 ; p=0.0090$, resp., Figure 5(a)); no significant differences between subgroups at this time point were found $(q=$ $0.5363, p>0.999)$.

As for the prefrontal cortex, "high-floaters" but not "low-floaters" showed significant increase of brain GSK3 $\beta$ mRNA on Day 5, as compared to session on Day 2 ("high-floaters": $q=3.793 ; p=0.0489$; "low-floaters" $q=0.3287 ; p$ $=0.9955$, resp., Tukey's test, Figure $5(b))$. No significant differences between the subgroups were revealed in this measure $(q=0.4696 ; p>0.9999, q=$ $0.4881 ; p>0.9999$, and $q=2.856 ; p=0.1695$; Figure $5(b))$. No other significant group differences were found.

No significant correlation was found between GSK3 $\beta$ mRNA levels in the hippocampus and the duration of floating behaviour on Day 1, Day 2, or Day $3(r=-0.06993 ; p=0.8346, r=-0.2545 ; p=0.4511$, and $r=0.008801 ; p=$ 0.9790 , resp., Spearman; Figure 5(c)). Spearman correlation analysis showed a significant correlation between mRNA levels of GSK3 $\beta$ in the prefrontal cortex and the duration of floating on Day $5(r=0.5944 ; p=0.0457$, 
Spearman) but not on Day 1 nor on Day $2(r=-0.9524 ; p=0.8401$ and $r=$ $0.1405 ; p=0.6449$, Spearman; Figure 5(d)).

Two-way ANOVA showed no significant differences in ratio pS9-GSK3 $\beta /$ total GSK-3 in the hippocampus on Day 1, Day 2, and Day $5(F=0.04540 ; p=$ 0.8324, two-way ANOVA; Figure 5(e)). At testing session on Day 5, as compared to session on Day 1 , this measure was significantly decreased in the hippocampus $(q=4.209 ; p=0.0247$ and $q=5.136 ; p=0.0043$, resp., Tukey's test; ANOVA; Figure 5(e)) and in the prefrontal cortex ( $q=4.389 ; p=$ 0.0184 and $q=2.990 ; p=0.1675$; Figure 5(f)) of both "high-" and "lowfloaters." No significant differences between subgroups of "high-" and "lowfloaters" were revealed at all time points in the hippocampus $(q=0.1931 ; p$ $>0.9999, q=0.4485 ; p>0.9999$, and $q=0.05110 ; p>0.9999$, resp.; Figure $5(e))$ nor in the prefrontal cortex $(q=2.194 ; p=0.7747, q=0.6684 ; p=$ 0.9997 , and $q=0.9733 ; p=0.9968$, resp.; Figure 5(f)). No other significant group differences were found.

The effects of imipramine on floating behaviour and GSK3B function in the modified swim test

ANOVA repeated measurements test showed that while non treated control groups displayed a significant increase in floating behaviour from Day 2 to Day 5 , no such increase was found in mice dosed with a low dose of antidepressant imipramine $(q=5.386 ; p=0.0038$ and $q=2.058 ; p=0.3412$, resp., Tukey's test; Figure 6(a)) (see Supplementary Table 5). Of note, there was no difference between control and imipramine-treated mice on Day 1 (q $=1.017$ e 006; $p$ > 0.9999; Figure 6(a)) and on Day 2 ( $q=1.163 ; p>0.9999$; Figure 6(a)), suggesting that applied dose was too low to be effective in the classic protocols of Porsolt's test. GSK3 $\beta$ mRNA levels were significantly altered in the hippocampus and were not changed in the prefrontal cortex and between the groups $(F=20.05 ; p<0.0001$ and $F=6.164 ; p=0.0053$, resp., ANOVA: Figures $6(\mathrm{~b})$ and $6(\mathrm{c}))$. Tukey's post hoc test showed a significant decrease of this parameter in the hippocampus of imipraminetreated mice, as compared with pharmacologically naïve group, on Day 5 ( $q$ $=8.952 ; p<0.0001 ;$ Figure $6(b))$. There was no such difference found for the prefrontal cortex $(q=2.584 ; p=0.1765$; Figure $6(c))$. A ratio $p S 9-G S K 3 \beta /$ total 
GSK3 $\beta$ among the groups was significantly altered in the hippocampus and in the prefrontal cortex $(F=10.65 ; p=0.0005$ and $F=13.73 ; p=0.0001$, resp., ANOVA; Figures $6(\mathrm{~d})$ and 6(e)). Post hoc analysis showed a significant reduction of this measure in non treated mice as compared to intact animals $(q=5.989 ; p=0.0008$, Tukey's test; Figure $6(d))$, while no such difference was found between intact mice and imipramine-treated group $(q=1.425, p$ $=0.5792$; Figure $6(d)$ ), suggesting that antidepressant treatment preserved normal levels of ratio pS9-GSK3 $\beta$ /total GSK3 $\beta$ in this brain structure. In the prefrontal cortex, a ratio pS9-GSK3 $\beta /$ total GSK3 $\beta$ was decreased in both groups subjected to modified forced swim test, as compared to intact control $(q=5.779 ; p=0.0013$ and $q=7.108 ; p=0.0001$; Figure $6(e))$; there was no difference between the latter groups $(q=1.904 ; p=0.3849$; Figure $6(\mathrm{e}))$.

\section{Discussion}

While repeated exposure to adversities including swimming under inescapable conditions has previously been used to achieve more robust depressive phenotypes by increasing the intensity of stress $[1,20,21]$, the protocol of repeated swimming proposed here was designed on a different basis. We have hypothesized that floating behaviour in additional swimming session of Porsolt's test in the mouse that is postponed from the initial noxious experience of swimming might be a better predictor of individual depressive-like behavioural and molecular changes than when measured in the original commonly used protocols of this model, as it likely implements a component of cognitive processing of adverse experiences. Present results support this assumption showing that similar factors to those that are crucial for acquisition of contextual memories in experimental models of learning, such as re-exposure to the context of testing, timing between challenge, and development of behavioural response [52, 53], are also important for the depressive-like behavioural and molecular effects in the repeated swim test with delayed testing. Moreover, modification of the swim test applied here has enabled us to differentiate the individual animal's susceptibility to a depressive-like state that so far was not achieved with the original Porsolt test. Together, our data provide evidence for a higher accuracy of the 
modified swim test in modeling depressive states and further support the hypothesis that enhanced contextual learning and consolidation of contextual memories impact the development of a depressive-like response.

Despite consideration that during delayed swim session, stress-related recall of an initial adverse experience may be extinguished and consequently animals display decreased floating behaviour, we found an opposite effect. Floating behaviour was further increased during the postponed swimming session and was prevented by antidepressant treatment of mice with a low dose of imipramine. Importantly, this potentiation of floating behaviour was evident in the very first minutes of testing and did not develop during the additional stressful swimming experience, suggesting that the animals were prone to floating already by the initial exposure to the additional swim test. The data reported here are in line with previously obtained findings, which showed that memory attenuation by various compounds, including NMDAreceptor and protein synthesis blockers, as wells as acetylcholinesterase inhibitors, may reduce floating behaviour, thus suggesting a role of contextual conditioning during repeated forced swimming [13, 54, 55]. Floating in the Porsolt test might therefore be called "learned immobility" and considered as an adaptive process that has been discussed in the literature since the introduction of this paradigm [56]. In this light, changes in GSK3 $\beta$ activities in the hippocampus and prefrontal cortex during forced swimming could provide further input in the interpretation of a physiological significance of floating behaviour in the mouse during this procedure.

The potentiation of behavioural despair on Day 5 was accompanied by an upregulation of GSK3 $\beta$, both a mediator of synaptic plasticity processes and a marker of a distress and depressive state in the brain $[21,25,28,30]$. The lack of changes in GSK-3alpha mRNA suggests specificity of the observed enhanced expression of GSK3 $\beta$. While analysis across experimental groups revealed elevated expression of GSK3 $\beta$ in the hippocampus, but not in the prefrontal cortex, a differentiation of mice by floating behaviour into subgroups according to their scores of depressive-like behaviour showed that animals defined as "high-floaters" displayed upregulated GSK3 $\beta$ in the prefrontal cortex as compared to "low-floaters" with a correlation between 
the duration of floating at Day 5 and GSK3 $\beta$ expression. Interestingly, there was no difference in GSK3 $\beta$ expression in the hippocampus between "highfloaters" and "low-floaters" as well as no correlation between hippocampal GSK3 $\beta$ mRNA and floating behaviour. Our results suggest that both hippocampus and prefrontal cortex are implicated in the formation of a depressive-like state in mice exposed to the modified swim test, but circuitry's function or/and dynamics of involvement in these processes are likely to be different. This is in line with other studies that suggest differential roles of these limbic structures in the mechanisms of behavioural despair, fear memories, and learned helplessness. Studies in recent years dissected the roles of particular regions of prefrontal cortex in the control of activity of amygdala during fear learning and its dependence on input from the hippocampus at early stages of consolidation, while distinct areas of the hippocampal formation were shown to be involved in long-term storage of contextual memory and stress response [35, 57-62]. Based on this evidence, similar GSK3 $\beta$ expression of "high-floaters" and "low-floaters" in the hippocampus may be interpreted as a molecular signature of a similar context recall in two groups that is not related to the development of a "depressive-like state." In contrary, given above-discussed role of the prefrontal cortex and GSK3 $\beta$ in the mechanism of stress and depression, elevated expression of this gene in the prefrontal cortex exclusively in a "high-floaters" group might be regarded as a molecular correlate of a depressive-like state in mice which are predisposed to this condition.

Reported behavioural and molecular changes were found on Day 5, but not in the testing protocols of the original Porsolt paradigm. Furthermore, they were largely prevented by a low dose of imipramine, which did not alter floating behaviour in the classic variants of the swim test, but in our modified protocol with a delayed session. Our finding suggests specific sensitivity of the neurobiological processes preceding the delayed swim test and their relevance for the pathophysiological mechanism of depressive-like states. Moreover, this pharmacologically validates our modified swim test as a model of a depressive-like state in rodents and suggests its improved sensitivity to antidepressant effectiveness of novel compound compared to commonly employed protocols of Porsolt's test. 
Finally, administration of imipramine precluded changes in the GSK3 $\beta$ both at the gene and protein level in the hippocampus, but not in the prefrontal cortex, further underscoring a differential role of these brain structures in the pathophysiology of depressive-like behaviour of helplessness and despair associated with this paradigm. Evidence suggests differential dynamics of gene and protein expression of GSK3 $\beta$ as well as compensatory changes in interrelated structures of the limbic system, under various conditions related to stress and learning of adverse memories [25, 26, 63] which are likely to explain distinct changes in brain GSK3 $\beta$ of imipraminetreated mice.

Increased GSK3 $\beta$ mRNA expression in the hippocampus and prefrontal cortex of mice subjected to repeated swimming was accompanied by a substantial reduction of an inactive form of GSK3 $\beta$, its phosphorylated form, and the pGSK3 $\beta / G S K 3 \beta$ ratio, as well-established molecular correlates of distress and depressive conditions $[64,65]$. The profound decrease of this ratio in both the hippocampus and prefrontal cortex further emphasizes the role of repeated swimming in the development of depressive-like behavioural and molecular changes observed in current study. Besides, while phosphorylation of GSK3 $\beta$ at 9-serine is considered to be the main regulatory mechanism of GSK3 $\beta$ function, based on the recent literature [66, 67], it might be hypothesized that an increase of GSK3 $\beta$ activities via phosphorylation at Tyr216 may occur under current experimental conditions and interfere with changes of this kinase at the gene and protein level reported here. This could be addressed in the next experiments with the modified swim test.

The pGSK3 $\beta / G S K 3 \beta$ ratio was similar on Day 2 and Day 5 of testing and no differences in these measures were found between "high-" and "low-" floaters, possibly reflecting limited sensitivity of this approach. As discussed above, the discrepancy between gene and protein parameters of GSK3 $\beta$ function on Day 2 and in the effects of imipramine on the prefrontal cortex might also be due to distinct dynamics of changes of gene expression and GSK3 $\beta$ phosphorylation and compensatory effects. We recently showed that while mice sacrificed $24 \mathrm{~h}$ after swim in the modified swimming test display 
no changes in GSK3 $\beta$ at the gene expression level, they have elevated GSK3 $\beta$ protein levels, suggesting that gene and protein changes in GSK3 $\beta$ may not always occur in parallel (Shevtsova and Markova, unpublished data). Generally, these results demonstrate that repeated but not single exposures to a swim test may result in an increase of activity of brain GSK3 $\beta$ at the protein level. Rapid changes of GSK3 $\beta$ activities on the gene and protein levels were previously reported; they were found as early as less than 10 min after challenge, while frequently fading away $24 \mathrm{~h}$ thereafter [26, $63,66]$.

A series of experiments that addressed the role of re-exposure of mice to a context of testing and timing in our modified swim test revealed their pivotal importance for the occurrence of behavioural and molecular changes observed in this paradigm. Study with a replacement of a swim session with an exposure to the context of testing on Day 2 and Day 5 has shown similar increases in floating and GSK3 $\beta$ gene expression to those observed in a conventional protocol of this model. These data, together with the finding that the omission of intermediate swimming or context exposure on Day 2 prevented an increase in floating, demonstrate crucial role of contextual components in both behavioural and molecular depressive-like features, which are induced in our swim model. A "premature" exposure to swimming and analysis of GSK3 $\beta$ changes on Day 3 showed a lack of changes that were typical for a conventional swim testing with a sacrifice on Day 5, thus pointing to the critical role of timing in the development of depressive-like behavioural and molecular features. These findings indicate that analogous to the processes of consolidation of learning experiences in mouse models of contextual memory $[52,53]$, the formation of a depressive-like phenotype requires a "consolidation phase." However, this hypothesis remains to be further explored assessing additional markers of molecular, structural, and functional plasticity. Such studies are in progress.

In summary, given the well-known role of GSK3 $\beta$ in neuronal and synaptic plasticity related to learning and memory on one hand and its importance as a factor of distress and maladaptation on the other hand, it may be speculated that GSK3 $\beta$ mediate pathologically enhanced consolidation of 
memories associated with adverse experience resulting in a depressive-like phenotype. Further studies are required to elucidate the role and the mechanism of enhanced learning in the context of adversity as the neurobiological basis of individual predisposition to depressive illness.

\section{Conclusions}

Taken together, the modified paradigm we propose offers a practical approach to study the role of enhanced cognitive processing in the pathophysiological mechanisms of depression and allows dissect interindividual differences in the susceptibility to this condition in mice. While classical versions of Porsolt's test, commonly regarded as tests for a depressive-like behaviour, do not per se mimic this condition, modified swim procedure does evoke the neurobiological changes that are relevant to a depressive-like state as proposed here. Hence, the modified swim test can be comparable to chronic depression paradigms, for example, chronic stress, learned helplessness, and similar models, which possess substantial construct validity and etiological relevance that cannot be attributed to short behavioural tests for a depressive-like behaviour, like "classic" Porsolt's and tail suspension tests. Importantly, proposed model, for the first time, may enable a tracking of an exact temporal relationship between an event that induces a depressive-like state and a development its molecular/neurobiological trace in dynamics that in such sense is virtually impossible with chronic models of depression. Given this and also minimal experimental work requirement of the new model proposed here, we believe that the modified swim test may provide a methodological solution for combining the advantages of chronic models of depression, while overcoming their disadvantages of highly demanding resources they typically require, as well as improving laboratory animal welfare.

\section{Acknowledgements}

The authors' work reported here was also supported by the European Community (EC: AGGRESSOTYPE FP7/no. 602805). The authors would also like to thank RFBR 11-04-01411, 16-34-01165 for support of this study. This 
publication was supported by the Open Access Publication Fund of the University of Wuerzburg.

\section{Competing Interests}

The authors declare that they have no competing interests.

\section{Authors' Contributions}

Tatyana Strekalova and Nataliia Markova are equally contributed.

\section{References}

[1] G. P. Chrousos, and P. W. Gold, "The concepts of stress and stress system disorders. Overview of physical and behavioural homeostasis", JAMA, vol. 267, no. 9, pp. 1244-1252, 1992.

[2] A. T. Beck, A. J. Rush, A. F. Shaw, and G. Emery, "Cognitive Therapy of Depression," New York: Guilford, vol. 1, no. 1, pp. 1-72, 1979.

[3] L. Clark, S. R. Chamberlain, and B. J. Sahakian, "Neurocognitive Mechanisms in Depression: Implications for Treatment", Annals and Reviews in Neuroscience, vol. 32, no. 7, pp. 57-74, 2009.

[4] Á. Flores, V. Valls-Comamala, G. Costa, R. Saravia, R. Maldonado, and F. Berrendero, "The hypocretin/orexin system mediates the extinction of fear memories", Neuropsychopharmacology, vol. 39, no. 12, pp. 2732-2741, 2014.

[5] P. E. Gold, and D. L. Korol, "Making memories matter", Frontiers integrative neuroscience, vol. 1, no. 12, 2012.

[6] D. M. Diamond, C. R. Park, and J. C. Woodson, "Stress generates emotional memories and retrograde amnesia by inducing an endogenous form of hippocampal LTP”, Hippocampus, vol. 14, no. 1, pp. 281-291, 2004.

[7] K. M. Myers, and M. Davis, "Mechanisms of fear extinction", Molecular Psychiatry, vol. 2007, no. 12, pp. 120-150, 2007. 
[8] A. Holmes, and N. Singewald, "Individual differences in recovery from traumatic fear", Trends in Neuroscience, vol. 36, no. 1, pp. 23-31, 2013.

[9] J. F. Cryan, and A. Holmes, "Model organisms: The ascent of mouse: advances in modelling human depression and anxiety", Nature Reviews Drug Discovery, vol. 4, no. 9, pp. 775-790, 2005.

[10] D. H. Overstreet, "Modeling Depression in Animal Models", Psychiatric Disorders, vol. 829, no. 1, pp. 125-144, 2012.

[11] P. Willner, and C. Belzung, "Treatment-resistant depression: are animal models of depression fit for purpose?" Psychopharmacology, vol. 232, no. 19, pp. 3473-3495, 2015.

[12] R. D. Porsolt, M. Le Pichon, and M. Jalfre, "Depression: a new animal model sensitive to antidepressant treatments", Nature, vol. 266, no. 5604, pp. 730-732, 1977.

[13] J. M. De Pablo, A. Parra, S. Segovia, and A. Guillamón, "Learned immobility explains the behaviour of rats in the forced swimming test", Physiology and Behaviour, vol. 46, no. 2, pp. 229-237, 1989.

[14] O. V. Bogdanova, S. Kanekara, K. E. D'Ancid, and P. F. Renshawa, "Factors influencing behaviour in the forced swim test", Physiology and Behaviour, vol. 118, no. 13, pp. 227-239, 2013.

[15] J. F. Cryan, R. J. Valentino, and I. Lucki, "Assessing substrates underlying the behavioural effects of antidepressants using the modified rat forced swimming test", Neuroscience Biobehavioural Reviews, vol. 29, no. 1, pp. 547-569, 2005.

[16] U. Schmidt, S. F. Kaltwasser, and C. T. Wotjak, "Biomarkers in posttraumatic stress disorder: overview and implications for future research", Disorder Markers, vol. 25, no. 1, pp. 43-54, 2013.

[17] J. M. H. M. Reul, "Making memories of stressful events: a journey along epigenetic, gene transcription, and signaling pathways", Frontiers in Psychiatry, vol. 2014, no. 1, 2014. 
[18] S.G. Anagnostaras, G. D. Gale, and M. S. Fanselow, "Hippocampus and contextual fear conditioning: recent controversies and advances", Hippocampus, vol. 11, no. 1, pp. 8-17. 2001.

[19] C. Katche, M. Cammarota, and J. H. Medina, "Molecular signatures and mechanisms of long-lasting memory consolidation and storage", Neurobiology of Learning and Memory, vol. 11, no. 106, pp. 40-47, 2013.

[20] T. E. Krahe, C. C. Filgueiras, and S. L. Schmidt, "Effects of rotational side preferences on immobile behaviour of normal mice in the forced swimming test", Progress in Neuropsychopharmacology and Biological Psychiatry, vol. 26, no. 1, pp. 169-176, 2002.

[21] B. Lee, I. Shim, H. Lee, and D. H. Hahm, "Effect of ginsenoside Re on depression- and anxiety-like behaviours and cognition memory deficit induced by repeated immobilization in rats", Journal of Microbiology and Biotechnology, vol. 22, no. 5, pp. 708-720, 2012.

[22] J. D. McBride, A. J. Jenkins, X. Liu, B. Zhang, K. Lee, W. L. Berry, R. Janknecht, C. T. Griffin, C. E. Aston, T. J. Lyons, J. J. Tomasek, and J. X. Ma, "Elevated circulation levels of an antiangiogenic SERPIN in patients with diabetic microvascular complications impair wound healing through suppression of Wnt signaling", Journal of Investigational Dermatology, vol. 134, no. 6, pp. 1725-1134, 2014.

[23] M. A. Mines, C. J. Yuskaitis, M. K. King, E. Beurel, and R. S. Jope, "GSK3 influences social preference and anxiety-related behaviours during social interaction in a mouse model of fragile X syndrome and autism", PLoS One, vol. 5, no. 306, 2010.

[24] W. Yu, J. Wu, F. Cai, J. Xiang, W. Zha, D. Fan, S. Guo, Z. Ming, and C. Liu, "Curcumin alleviates diabetic cardiomyopathy in experimental diabetic rats," PLoS One, vol. 7, no. 12, 2012.

[25] L. Chen, J. Dai, Wang Z, Zhang H, Huang Y, and Zhao Y, "Ginseng Total Saponins Reverse Corticosterone-Induced Changes in Depression-Like Behaviour and Hippocampal Plasticity-Related Proteins by Interfering with 
GSK-3 $\beta$-CREB Signaling Pathway", Evidence Based Complementary Alternative Medicine, vol. 2014, no. 506735, 2014.

[26] Y. C. Chen, Q. R. Tan, W. Dang, H. N. Wang, R. B. Zhang, Z. Y. Li, H. Lin, and R. Liu, "The effect of citalopram on chronic stress-induced depressivelike behaviour in rats through GSK3 $\beta / \beta$-catenin activation in the medial prefrontal cortex", Brain Research Bulletin, vol. 88, no. 4, pp. 338-344, 2012.

[27] W. T. O'Brien, A. D. Harper, F. Jové, J. R. Woodgett, S. Maretto, S. Piccolo, and P. S. Klein, "Glycogen synthase kinase-3beta haploinsufficiency mimics the behavioural and molecular effects of lithium", Journal of Neuroscience, vol. 24, no. 30, pp. 6791-6798, 2004.

[28] R. Silva, A. R. Mesquita, J. Bessa, J. C. Sousa, I. Sotiropoulos, P. Leão, O. F. Almeida, and N. Sousa, "Lithium blocks stress-induced changes in depressive-like behaviour and hippocampal cell fate: the role of glycogensynthase-kinase-3beta", Neuroscience, vol. 152, no. 3, pp. 656-69, 2008.

[29] M. Dahlhoff, A. Siegmund, Y. Golub, E. Wolf, F. Holsboer, and C. T. Wotjak, "AKT/GSK3 $3 /$ beta-catenin signalling within hippocampus and amygdala reflects genetically determined differences in post-traumatic stress disorder like symptoms", Neuroscience, vol. 169, no. 3, pp. 1216-1226, 2010.

[30] D. H. Oh, Y. C. Park, and S. H. Kim, "Increased glycogen synthase kinase$3 \beta$ mRNA level in the hippocampus of patients with major depression: a study using the stanley neuropathology consortium integrative database", Psychiatry Investigation, vol. 7, no. 3, pp. 202-207, 2010.

[31] K. Zhang, X. Song, Y. Xu, X. Li, P. Liu, N. Sun, X. Zhao, Z. Liu, Z. Xie, and J. Peng, "Continuous GSK3 $\beta$ overexpression in the hippocampal dentate gyrus induces prodepressant-like effects and increases sensitivity to chronic mild stress in mice", Journal of Affective Disorders, vol. 146, no. 1, pp. 45-52, 2013.

[32] M. Szymańska, A. Suska, B. Budziszewska, L. Jaworska-Feil, A. BastaKaim, M. Leśkiewicz, M. Kubera, A. Gergont, S. Kroczka, M. Kaciński, and W. 
Lasoń, "Prenatal stress decreases glycogen synthase kinase-3 phosphorylation in the rat frontal cortex", Pharmacological Reports, vol. 61, no. 4, pp. 612-620, 2009.

[33] J. A. Grizzell, A. larkov, R. Holmes, T. Mori, and V. Echeverria, "Cotinine reduces depressive-like behaviour, working memory deficits, and synaptic loss associated with chronic stress in mice", Behavioural Brain Research, vol. 6, no. 268, pp. 55-65, 2014.

[34] D. Kapfhamer, K. H. Berger, F. W. Hopf, T. Seif, V. Kharazia, A. Bonci, and U. Heberlein, "Protein Phosphatase $2 a$ and glycogen synthase kinase 3 signaling modulate prepulse inhibition of the acoustic startle response by altering cortical M-Type potassium channel activity," Journal of Neuroscience, vol. 30, no. 26, pp. 8830-8840, 2010.

[35] T. F. Giustino, and S. Maren, "The Role of the Medial Prefrontal Cortex in the Conditioning and Extinction of Fear", Frontiers in Behavioural Neuroscience, vol. 9, no. 298, 2015.

[36] D. Lopresto, P. Schipper, and J. R. Homber, "Neural circuits and mechanisms involved in fear generalization: Implications for the pathophysiology and treatment of post-traumatic stress disorder", Neurosci Biobehav Rev, vol. 60, no. 1, pp. 31-42, 2016.

[37] I. Izquierdo, C. R. Furini, and J. C. Myskiw, "Fear Memory", Physiological Reviews, vol. 96, no. 2, pp. 695-750, 2016.

[38] W. T. O'Brien, and P. S. Klein, "Regulation of glycogen synthase kinase-3 in patients with affective disorders", Biological Psychiatry, vol. 61, no. 2, pp. 139-141, 2007.

[39] F. Karege, N. Perroud, S. Burkhardt, R. Fernandez, E. Ballmann, R. La Harpe, and A. Malafosse, "Protein levels of $\beta$-catenin and activation state of glycogen synthase kinase-3 $\beta$ in major depression. A study with postmortem prefrontal cortex", Journal of Affective Disorders, vol. 136, no. 1-2, pp. 185188, 2012. 
[40] T. Strekalova, M. Evans, A. Chernopiatko, Y. Couch, J. Costa-Nunes, R. Cespuglio, L. Chesson, J. Vignisse, H. W. Steinbusch, D. C. Anthony, I. Pomytkin, and K. P. Lesch, "Deuterium content of water increases depression susceptibility: the potential role of a serotonin-related mechanism", Behaviour Brain Research, vol.277, no. 1, pp. 237-244, 2015.

[41] E. Malatynska, H. W. Steinbusch, O. Redkozubova, A. Bolkunov, A. Kubatiev, N. B. Yeritsyan, J. Vignisse, S. Bachurin, and T. Strekalova, "Anhedonic-like traits and lack of affective deficits in 18-month-old C57BL/6 mice: Implications for modeling elderly depression", Experimental Gerontology, vol. 47, no. 8, pp. 552-564, 2012.

[42] J. Costa-Nunes, O. Zubareva, M. Araújo-Correia, A. Valença, C. A. Schroeter, J. L. Pawluski, J. Vignisse, H. Steinbusch, D. Hermes, M. Phillipines, H. M. Steinbusch, and T. Strekalova, "Altered emotionality, hippocampusdependent performance and expression of NMDA receptor subunit mRNAs in chronically stressed mice", Stress, vol. 17, no. 1, pp. 108-116, 2014.

[43] T. Strekalova, C. T. Wotjak, and M. Schachne, "Intrahippocampal administration of an antibody against the HNK-1 carbohydrate impairs memory consolidation in an inhibitory learning task in mice", Molecular Cellular Neuroscience, vol. 17, no. 6, pp. 1102-1113, 2001.

[44] Y. Couch, D. C. Anthony, O. Dolgov, A. Revischin, B. Festoff, A. I. Santos, H. W. Steinbusch, and T. Strekalova, "Microglial activation, increased TNF and SERT expression in the prefrontal cortex define stress-altered behaviour in mice susceptible to anhedonia", Brain Behaviour Immuninty, vol. 36, no. 29, pp. 136-143, 2013.

[45] B. H. Cline, J. P. Costa-Nunes, R. Cespuglio, N. Markova, A. I. Santos, Y. V. Bukhman, A. Kubatiev, H. W. M. Steinbusch, K. P. Lesch, and T. Strekalova, "Dicholine succinate, the neuronal insulin sensitizer, normalizes behaviour, REM sleep, hippocampal pGSK3 $\beta$ and mRNAs of NMDA receptor subunits in mouse models of depression", Frontiers in Behavioural Neuroscience, vol. 6, no. 9, pp. 37-55, 2015. 
[46] J. P. Costa-Nunes, B. H. Cline, M. Araújo-Correia, M. Valença, N. Markova, O. Dolgov, A. Kubatiev, N. Yeritsyan, H. W. Steinbusch, and T. Strekalova, "Animal models of depression and drug delivery with food as an effective dosing method: evidences from studies with celecoxib and dicholine succinate", Biomed Research International, vol. 3, no. 1, 2015.

[47] N. M. Derks, M. Müller, B. Gaszner, D. T. Tilburg-Ouwens, E. W. Roubos, and L. T. Kozicz, "Housekeeping genes revisited: different expressions depending on gender, brain area and stressor", Neuroscience, vol. 156, no. 2, pp. 305-309, 2008.

[48] M. Borsoi, C. B. Antonio, L. G. Müller, A. F. Viana, V. Hertzfeldt, P. S. Lunardi, C. Zanotto, P. Nardin, A. P. Ravazzolo, S. M. Rates, and C. A. Gonçalves, "The hydrophobic dipeptide Leu-lle inhibits immobility induced by repeated forced swimming via the induction of BDNF", Behavioural Brain Research, vol. 220, no. 2, pp. 271-280, 2011.

[49] E. M. Jennings, B. N. Okine, W. M. Olango, M. Roche, and D. P. Finn, "Repeated forced swim stress differentially affects formalin-evoked nociceptive behaviour and the endocannabinoid system in stress normoresponsive and stress hyper-responsive rat strains", Neuroscience, vol. 220, no. 1, pp. 109-118, 2012.

[50] A. G. Gornall, C. J. Bardawill, and M. M. David, "Determination of serum proteins by means of the biuret reaction," Journal of Biological Chemistry, vol. 177, no. 8, pp. 751-766, 1949.

[51] T. Strekalova T, D.C. Anthony, O. Dolgov, K. Anokhin, and A. Kubatiev, H.M. Steinbusch, and C. Schroeter, "The differential effects of chronic imipramine or citalopram administration on physiological and behavioural outcomes in naïve mice", Behavioural Brain Research, vol. 245, no. 2, pp. 101-116, 2013.

[52] P. Joshua, J. P. Johansen, C. K. Cain, L. E. Ostroff, and J. E. LeDoux, "Molecular mechanisms of fear learning and memory", Cell, vol. 147, no. 3, pp. 509-524, 2011. 
[53] A. M. Miller, L. C. Vedder, L. M. Law, and D. M. Smith, "Cues, context, and long-term memory: the role of the retrosplenial cortex in spatial cognition", Frontiers in Human Neurosciences, vol. 8, no. 586, 2014.

[54] S. M. Korte, E. R. De Kloet, B. Buwalda, S. D. Bouman, and B. Bohus, "Antisense to the glucocorticoid receptor in hippocampal dentate gyrus reduces immobility in forced swim test", European Journal of Pharmacology, vol. 301, no. 1-3, pp. 19-25, 1996.

[55] N. Enginar, P. Yamantürk-Çelik, A. Nurten, and D. B. Güney, "Learning and memory in the forced swimming test: effects of antidepressants having varying degrees of anticholinergic activity", Naunyn Schmiedebergs Archives of Pharmacology, vol. 1, no. 1, pp. 1-7, 2016.

[56] E. R. de Kloet, and M. L. Molendijk, "Coping with the Forced Swim Stressor: Towards Understanding an Adaptive Mechanism", Neural Plasticity, vol. 1, no. 1, pp 465-478, 2016.

[57] F. Sotres-Bayon, and G. J. Quirk, "Prefrontal control of fear: more than just extinction," Current Opinion in Neurobiology, vol. 20, no. 1, pp. 231-235, 2010.

[58] M. R. Warden, A. Selimbeyoglu, J. J. Mirzabekov, M. Lo, K. R. Thompson, S. Y. Kim, A. Adhikari, K. M. Tye, L. M. Frank, and K. A. Deisseroth, "Prefrontal cortex-brainstem neuronal projection that controls response to behavioural challenge", Nature, vol. 492, no. 1, pp. 428-432, 2012.

[59] H. S. Mayberg, M. Liotti, S. K. Brannan, S. McGinnis, R. K. Mahurin, P. A. Jerabek, J. A. Silva, J. L. Tekell, C. C. Martin, J. L. Lancaster, and P. T. Fox, "Reciprocal limbic-cortical function and negative mood: converging PET findings in depression and normal sadness", Journal of Psychiatry, vol. 156, no. 1, pp. 675-682, 1999.

[60] A. Etkin, "Functional neuroanatomy of anxiety: a neural circuit perspective", Current Topics in Behavioural Neuroscience, vol. 2, no. 1, pp. 251-277, 2010. 
[61] M. R. Milad, and G. J. Quirk, "Fear extinction as a model for translational neuroscience: ten years of progress", Annals of Reviews in Psychology, vol. 63, no. 1, pp. 129-151, 2012.

[62] M. Wang, Z. Perova, B. R. Arenkiel, and B. Li, "Synaptic modifications in the medial prefrontal cortex in susceptibility and resilience to stress", Journal of Neuroscience, vol. 34, no. 22, pp. 7485-7492, 2014.

[63] M. S. Roh, U. G. Kang, S. Y. Shin, Y. H. Lee, H. Y. Jung, Y. S. Juhnn, and Y. S. Kim, "Biphasic changes in the Ser-9 phosphorylation of glycogen synthase kinase-3beta after electroconvulsive shock in the rat brain", Progress in Neuropsychopharmacology and Biological Psychiatry, vol. 27, no. 1, pp. 1-5, 2003.

[64] J.-M. Beaulieu, X. Zhang, R. M. Rodriguiz, T. D. Sotnikova, M. J. Cools, W. C. Wetsel, R. R. Gainetdinov, and M. G. Caron, "Role of GSK3 $\beta$ in behavioural abnormalities induced by serotonin deficiency", PNAS, vol. 105 no. 4, pp. 1333-1338, 2008.

[65] G. Li, T. Liu, X. Kong, L. Wang, and X. Jin, "Hippocampal glycogen synthase kinase $3 \beta$ is critical for the antidepressant effect of cyclindependent kinase 5 inhibitor in rats", Journal of Molecular Neuroscience, vol. 54, no. 1, pp. 92-99, 2014.

[66] M. Medina, and F. Wandosell F. "Deconstructing GSK-3: The Fine Regulation of Its Activity", International Journal of Alzheimers Disorder. 2011.

[67] O. O'Leary, and Y. Nolan. "Glycogen synthase kinase-3 as a therapeutic target for cognitive dysfunction in neuropsychiatric disorders", CNS Drugs. Vol. 29, no 1, pp. 1-15, 2015.

[68] M. Shapira, A. Licht, A. Milman, C. G. Pick, E. Shohami, and H. EldarFinkelman. "Role of glycogen synthase kinase-3beta in early depressive behaviour induced by mild traumatic brain injury", Molecular and Cellular Neuroscience. vol. 34, no. 4, pp. 571-577, 2007. 


\section{Figure legends}

Figure 1. Schematic timeline of experiments. (a) Investigation of behavioural and molecular changes in the modified versus classical swimming paradigms. (b-f) Studies to address the impact of context, swimming, and timing of testing in behavioural and molecular changes induced in the modified swim test. (g) Investigation of behavioural and molecular effects of imipramine in the modified swim test. D1: Day 1; D2: Day 2; D3: Day 3; D5: Day 5.

Figure 2. Behavioural changes induced in the modified swim test and the role of context, swimming and timing of testing. (a) Mice exposed to the modified swim test $(n=14)$ showed an increase of total duration of floating. (b-d) An increase of duration of floating at the delayed testing session on Day 5 in comparison to the session on Day 2 was the pronounced during the first two-minute periods. (e) In comparison to the session on Day 2, mice subjected to the modified swim test have displayed significantly increased total duration of floating when the testing on Day 2 was replaced with exposure to a context of testing $(n=10)$ that was similar to the changes in regular modified swim test, but not when the testing on Day 2 was omitted $(n=9)$. Data are expressed in percent from mean values of floating on Day 1 for each group. (f) Mice displayed a significant increase in the total duration of floating on Day 3 as compared to Day 1 , but not Day $2(n=10) . * p<0.05$, ANOVA and Tukey's multiple comparisons test; see the text; Context D2: mice were exposed to a context of testing instead of swim session on Day 2; no testing D2: testing on Day 2 was omitted. D1: Day 1; D2: Day 2; D3: Day 3; D5: Day 5. Data are mean \pm SEM.

Figure 3. Brain expression of GSK3 $\beta$ in the modified swim test and the role of context, swimming and timing of testing. (a) In comparison to intact controls ( $n=11$ ), hippocampal GSK3 $\beta$ mRNA levels were significantly elevated only on Day $5(n=14)$, but not on Day $1(n=12)$ or Day $2(n=11)$. (b) In the prefrontal cortex, the GSK3 $\beta$ mRNA levels were not changed on Day 1, Day 2, or Day 5; group sizes as indicated above. As compared with intact mice $(n=6)$, there was no change in GSK3 $\beta$ mRNA in mice sacrificed $24 \mathrm{~h}$ after swim session on Day 5 in the hippocampus (c), nor in the 
prefrontal cortex (d); $n=14$ in each experimental group. (e) In comparison to intact controls $(n=10)$, hippocampal GSK3 3 mRNA levels were not significantly altered in mice tested and sacrificed on Day $3(n=6)$ nor in mice sacrificed on Day 5 without any behavioural manipulations $(n=6)$ or exposed to a context at testing on Day $5(n=6)$. ( $f$ ) In comparison to intact controls, GSK3 $\beta$ mRNA levels were not changed in the prefrontal cortex of mice exposed to testing on Day 3, nor in mice sacrificed on Day 5 without any behavioural manipulations, and significantly elevated in animals exposed to a context of testing on Day 5; group sizes as indicated above. As compared with intact mice ( $n=9)$, in mice exposed to the modified swim test $(n=13)$, there was no change in GSK-3alpha mRNA levels nor $(g)$ in the hippocampus, nor (h) in the prefrontal cortex. $* p<0.05$, ANOVA and Tukey's multiple comparisons test; see the text; +10 min: mice sacrificed $10 \mathrm{~min}$ after swim session; +24 h: mice sacrificed $24 \mathrm{~h}$ after swim session; D3: mice exposed to the swim session on Day 3 instead of a swim on Day 5; Cont D5: mice exposed to a context of testing instead of a swim session on Day 5; no swim D5: mice were not exposed to a swim nor a context of swimming test prior a sacrifice. D1: Day 1; D2: Day 2; D5: Day 5. Data are mean \pm SEM.

Figure 4. Brain levels of total and phosphorylated forms of GSK3 $\beta$ in the modified swim test. (a) In comparison to intact controls $(n=7)$, hippocampal level of total GSK3 $\beta$ was not significantly changed on Day $1(n=14)$, nor on Day $2(n=10)$ or on Day $5(n=12)$ of swim test. (b) In comparison to intact group, total level of GSK3 $\beta$ in the prefrontal cortex was significantly increased on Day 1 of swim test but not on Day 2 and Day 5; group sizes as indicated above. (c) In comparison to intact animals, hippocampal level of phosphorylated form of GSK3 $\beta$ was significantly decreased on Day 2 and Day 5 , but not on Day 1 of the test; group sizes as indicated above. (d) In comparison to intact mice, the levels of pS9- GSK3 $\beta$ in prefrontal cortex were not altered on Day 2 and Day 5 of swim test; group sizes as indicated above. In comparison to intact controls, ratio of total/pS9 GSK3 3 (e) in the hippocampus and ( $f$ ) in the prefrontal cortex was significantly decreased on Day 2 and Day 5, but not on Day 1; group sizes as indicated above. $* p<0.05$, ANOVA and Tukey's multiple comparisons test; see the text. D1: Day 1; D2: Day 2; D5: Day 5. Data are mean \pm SEM. 
Figure 5. Brain activities of GSK3 $\beta$ in "High"- and "Low-floaters" in the modified swim test. Mice were assigned to subgroups with "high-" $(n=7)$ or "low-" floaters $(n=7)$ using a mean of the groups as a criterion of a group formation. In comparison to intact controls, GSK3 $\beta$ mRNA (a) in the hippocampus and (b) in the prefrontal cortex was not significantly changed in either subgroup of mice on Day 1, Day 2, and Day 5 of swim test. (c) Hippocampal levels of GSK3 $\beta$ mRNA $(n=14)$ did not correlate with total duration of floating on Day 5 of swim test. (d) The levels of GSK3 $\beta$ mRNA in prefrontal cortex $(n=14)$ significantly correlated with the total duration of floating on Day 5 of a swim test. (e) In comparison to intact controls, hippocampal ratio of pS9 GSK3 $\beta$ /total GSK3 $\beta$ was significantly decreased both in "low" and "high" floating mice on Day 5, but not on Day 1 and Day 2. (f) In comparison to intact mice, the ratio of pS9 GSK3 $\beta$ /total GSK3 $\beta$ in prefrontal cortex was not significantly changed on Day 5 of swim test; size of groups as indicated above. $* p<0.05$, ANOVA and Tukey's multiple comparisons test; see the text; LowF: subgroup of mice classified as "lowfloaters"; HighF: subgroup of mice classified as "high-floaters." D1: Day 1; D2: Day 2; D5: Day 5. Data are mean \pm SEM.

Figure 6. The effects of imipramine on floating behaviour and brain GSK3 $\beta$ activities in the modified swim test. (a) Imipramine-treated mice $(n=14)$ showed no significant increase of floating behaviour as compared with control group $(n=14)$, from Day 2 to Day 5 . As compared to intact controls ( $n=13$ ), on Day 5, GSK3 $\beta$ mRNA of imipramine-treated mice was (b) decreased in the hippocampus and (c) not changed in the prefrontal cortex; group sizes as indicated above. (d) As compared to intact mice, imipraminetreated group had significantly increased ratio of total/pS9 of GSK3 $\beta$ in the hippocampus on Day 5; group sizes as indicated above. (e) As compared with intact mice, there were no significant changes the in ratio of pS9 GSK3 $\beta$ /total GSK3 $\beta$ in the prefrontal cortex of imipramine-treated mice on Day 5 of swim test; group sizes as indicated above. ${ }^{*} p<0.05$, ANOVA and Tukey's multiple comparisons test; see the text; Imi: imipramine-treated group; D1: Day 1; D2: Day 2; D5: Day 5. Data are mean \pm SEM. 
Modified swim test: adding a delayed session to the classic Porsolt test

\begin{tabular}{|c|c|c|c|}
\hline $\begin{array}{c}\text { D1 } \\
\text { Swimming }\end{array}$ & $\begin{array}{c}\text { D2 } \\
\text { Swimming }\end{array}$ & \multicolumn{2}{|c|}{$\begin{array}{c}\text { D5 } \\
\text { Swimming }\end{array}$} \\
\hline $\begin{array}{l}\text { Sacrifice } \\
+10 \mathrm{~min}\end{array}$ & $\begin{array}{l}\text { Sacrifice } \\
+10 \text { min }\end{array}$ & $\begin{array}{l}\text { Sacrifice } \\
+10 \text { min }\end{array}$ & $\begin{array}{c}\text { Sacrifice } \\
+24 \mathrm{~h}\end{array}$ \\
\hline
\end{tabular}

(a)

Modified protocol of swim test: impact of context, swimming, and timing

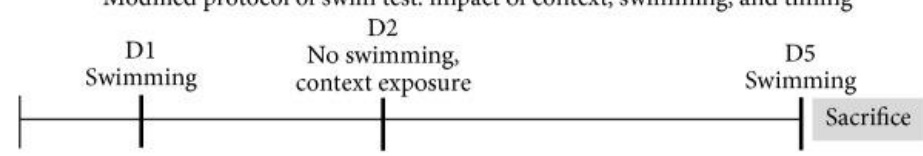

(b)

Modified protocol of swim test: impact of context, swimming, and timing D2

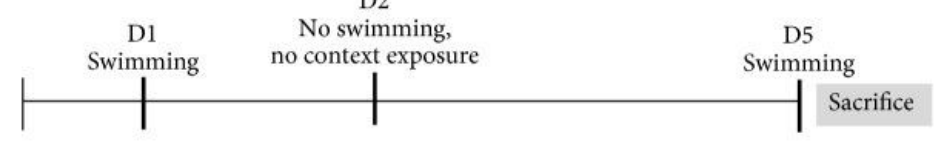

(c)

Modified protocol of swim test: impact of context, swimming, and timing

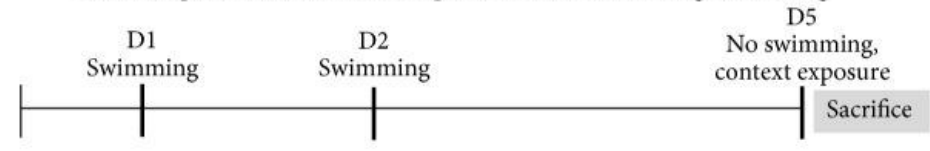

(d)

Modified protocol of swim test: impact of context, swimming, and timing

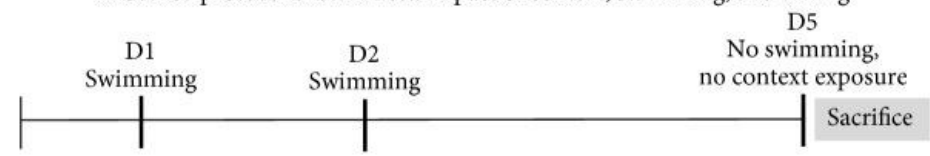

(e)

Modified protocol of swim test: impact of context, swimming, and timing

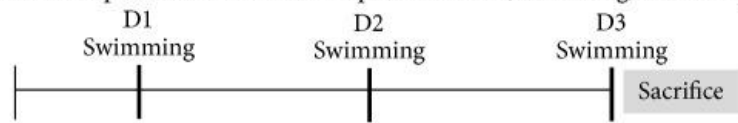

(f)

Modified swim test and effects of low dose of imipramine

Dosing with imipramine $(7.5 \mathrm{mg} / \mathrm{kg} /$ day with drinking water $)$ Control: drinking water

\begin{tabular}{|l|l|l|l}
-14 days & $\mathrm{D} 1$ & $\mathrm{D} 2$ & $\mathrm{D} 5$ \\
Swimming & Swimming & Swimming
\end{tabular}

(g)

\section{Figure 1}


DYNAMICS OF FLOATING
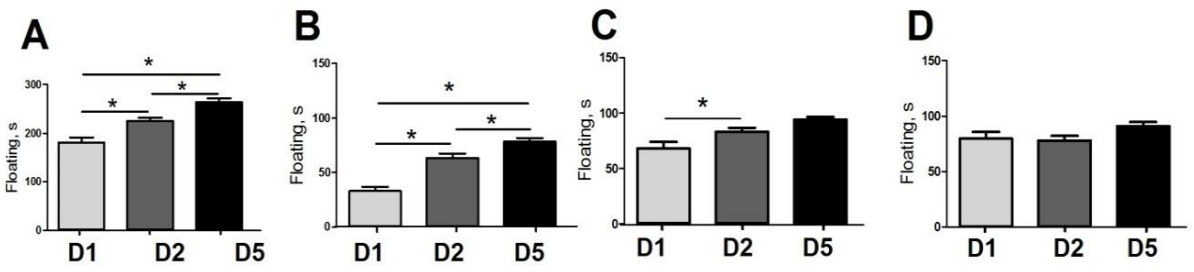

THE IMPACT OF CONTEXT, SWIMMING AND TIMING IN FLOATING
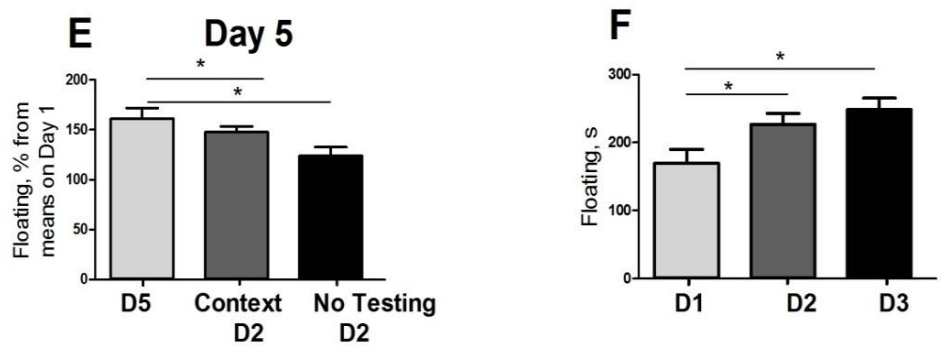

Figure 2 

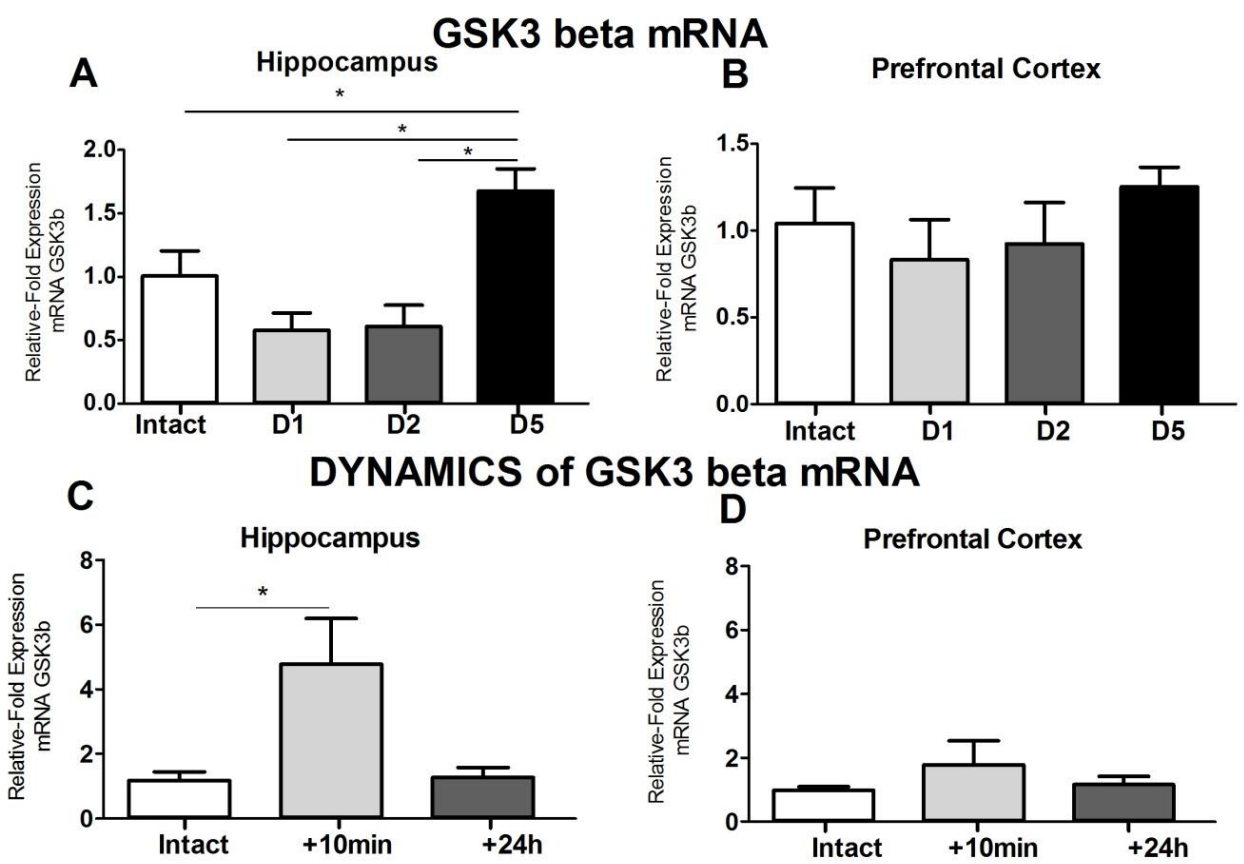

\section{THE IMPACT OF A CONTEXT, SWIMMING AND TIMING IN GSK3 beta EXPRESSION}

$\mathbf{E}$

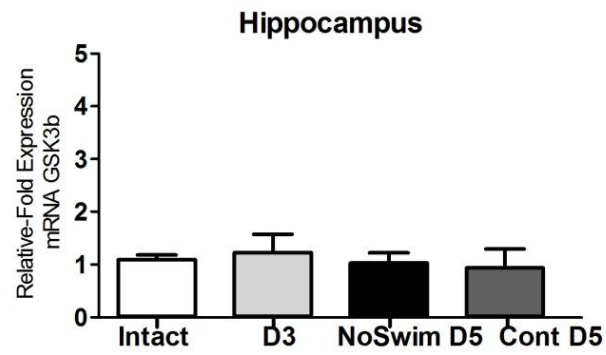

$\mathbf{F}$

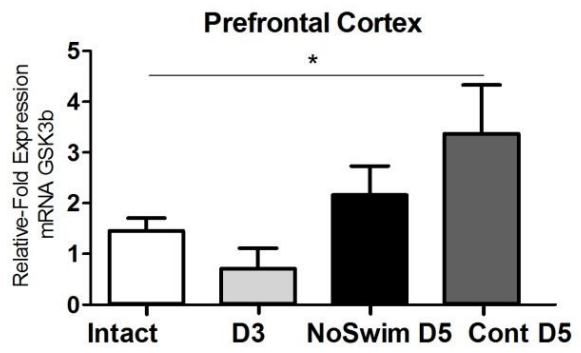

\section{GSK3 alpha mRNA}
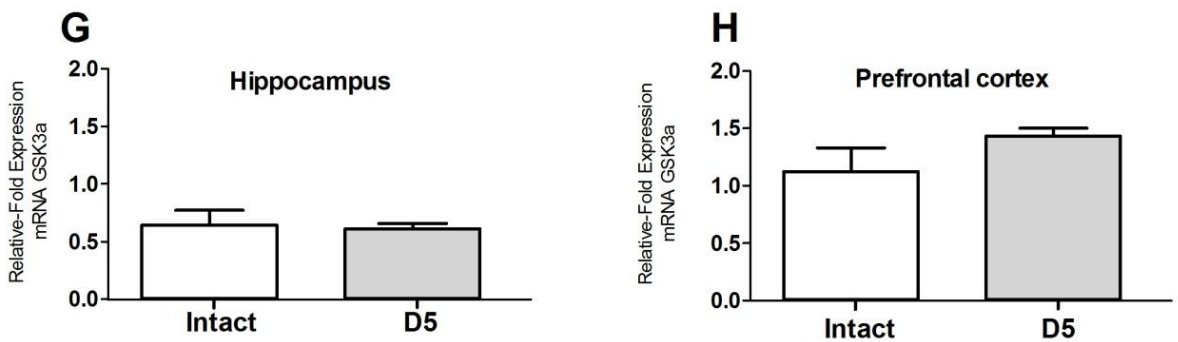

Figure 3 
TOTAL LEVELS OF GSK3 beta

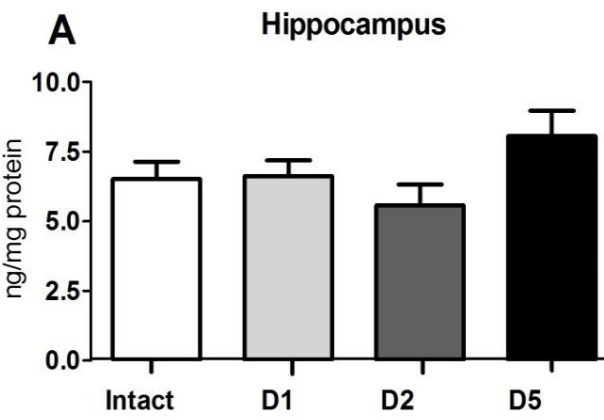

B Prefrontal Cortex

pS9- GSK3 beta
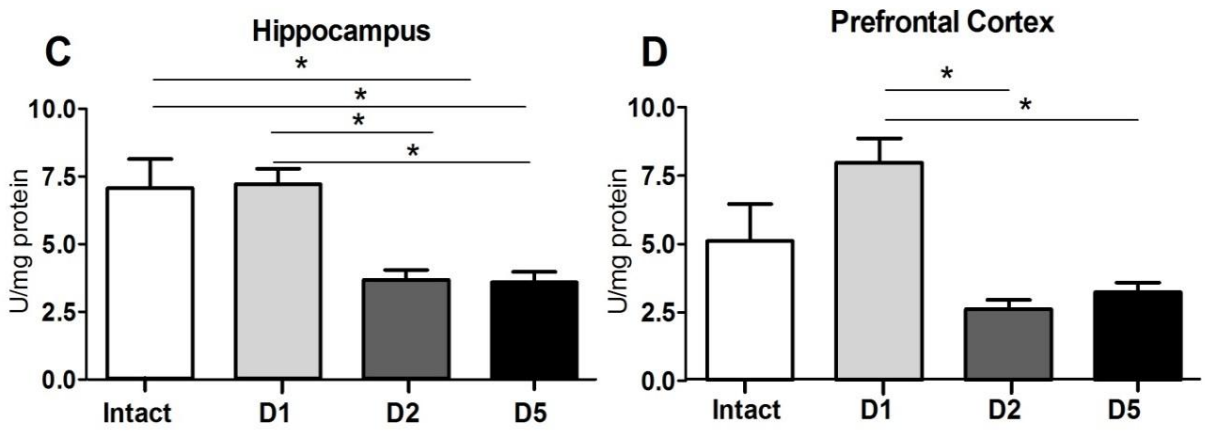

RATIO pS9- GSK3 beta: TOTAL GSK3 beta

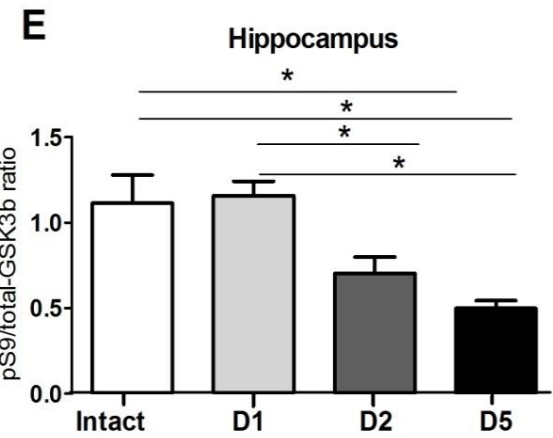

$\mathbf{F}$

Prefrontal Cortex

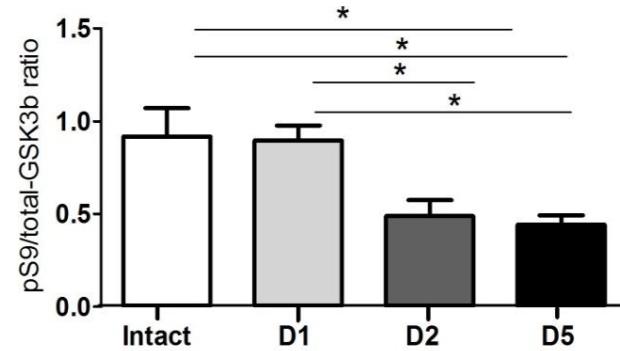

Figure 4 


\section{GSK3 beta mRNA}

A

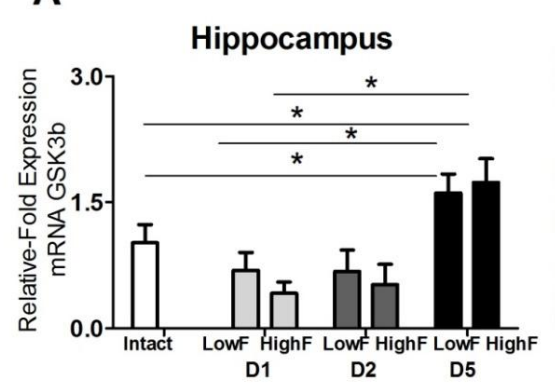

C

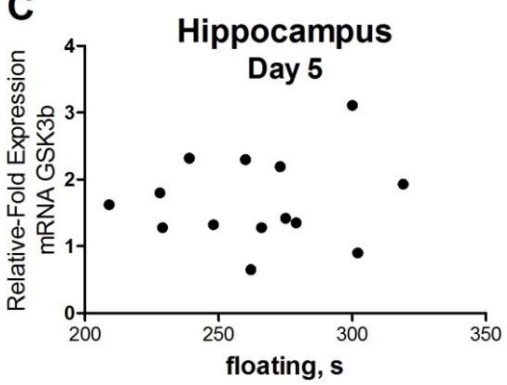

B
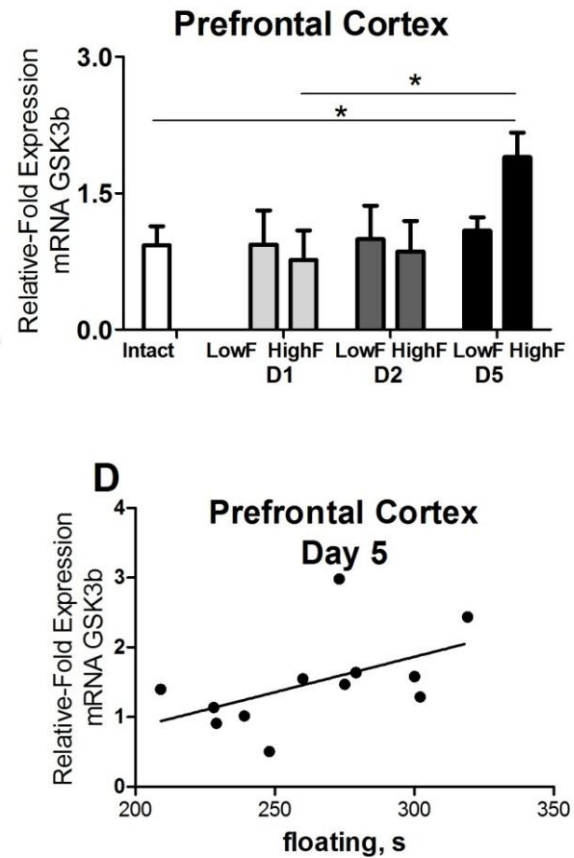

RATIO pS9- GSK3 beta: TOTAL GSK3 beta
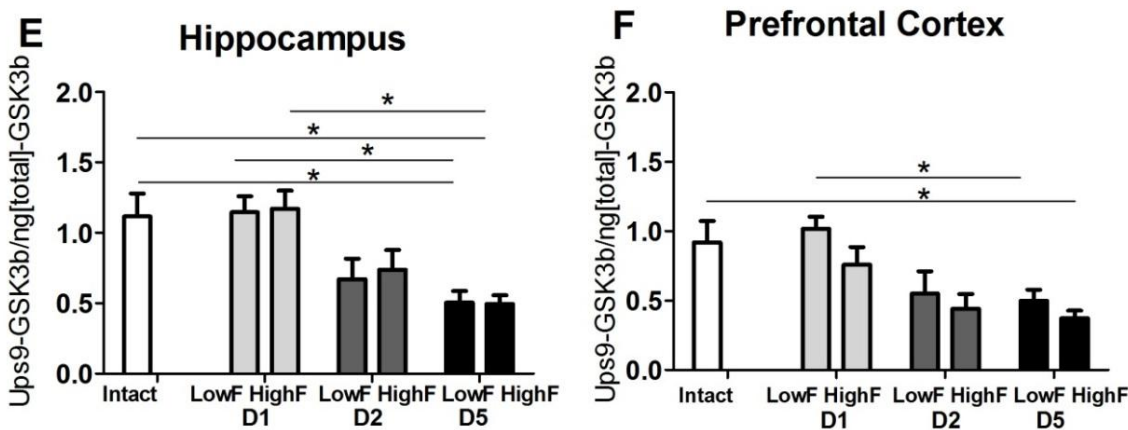

Figure 5 


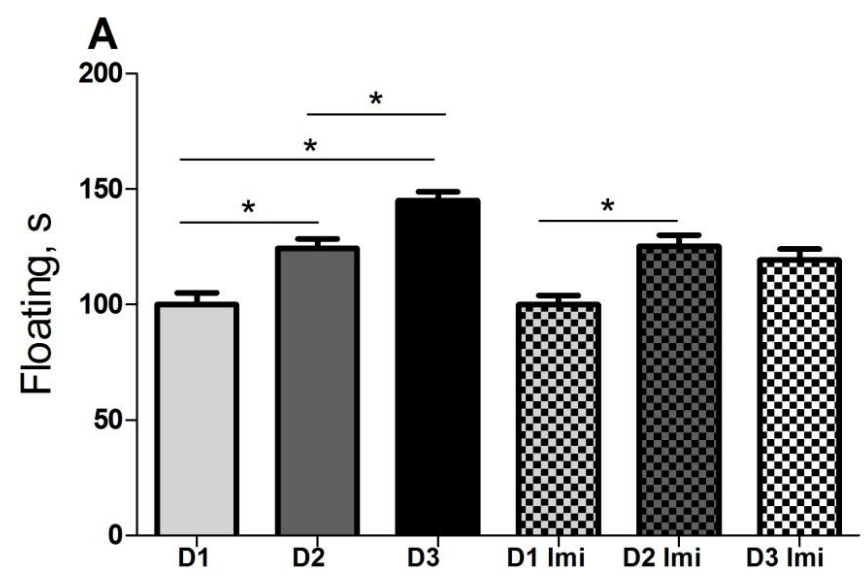

GSK3 beta mRNA
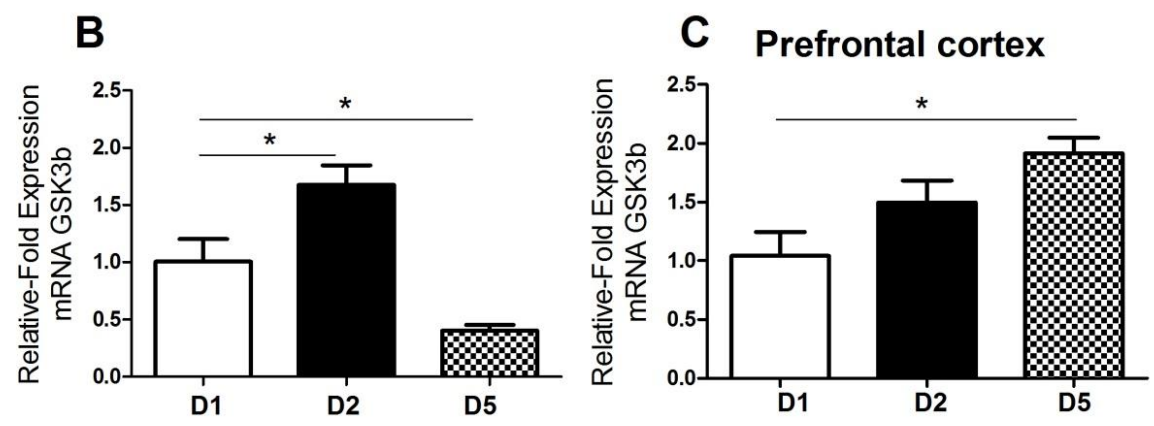

RATIO pS9- GSK3 beta: TOTAL GSK3 beta
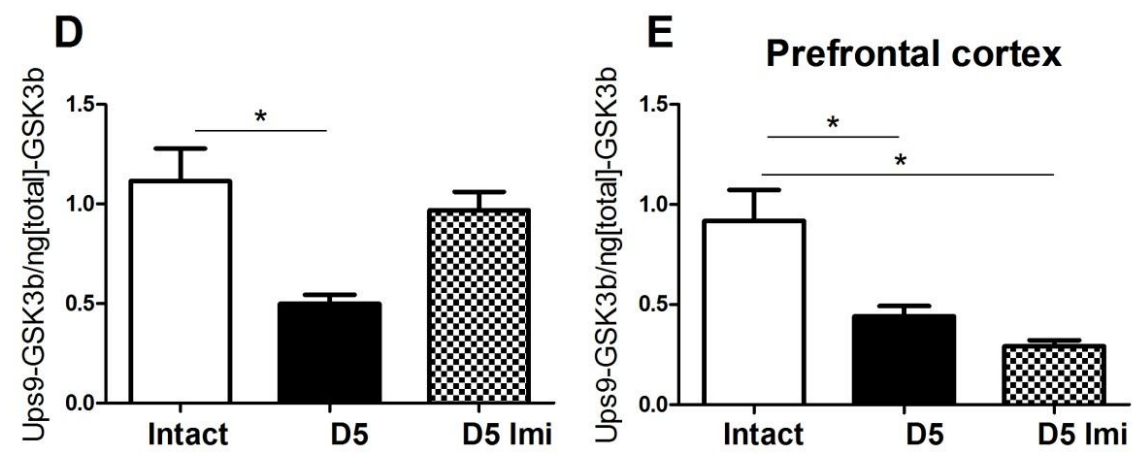

Figure 6 


\section{Supplementary data}

Table 1. SEQUENCES OF PRIMERS USED

\begin{tabular}{|l|l|l|}
\hline Gene & Forward primer 5'-3' & Reverse primer 5'-3' \\
\hline GAPDH & TGCACCACCAACTGCTTAG & GGATGCAGGGATGATGTTC \\
\hline GSK-3 alpha & AATCTTGGCCAGTCTGAGCT & TCAGTCCTGGTGAACTGTCC \\
\hline GSK-3 beta & TCCATTCCTTTGGAATCTGC & CAATTCAGCCAACACACAGC \\
\hline
\end{tabular}

RNA isolation and RT PCR

The qRT-PCR was performed in a $25 \mathrm{mcl}$ reaction volume containing a 10xPCR Buffer $(2.5 \mu \mathrm{l}), 25 \mathrm{mM} \mathrm{MgCl} 2(4 \mu \mathrm{l}), 10 \mathrm{mM}$ dNTPs $(2 \mu \mathrm{l})$, specific forward and reverse primers at $20 \mathrm{pmol} / \mu \mathrm{l}$ concentration $(1 \mu \mathrm{l}), \mathrm{cDNA}(2 \mu \mathrm{l})$, $5 \mathrm{u} / \mu \mathrm{l}$ Taq DNA polymerase $(1 \mu \mathrm{l})$ (Beagle, st. Petersburg, Russia), and $\mathrm{ddH} 2 \mathrm{O}(10 \mu \mathrm{l})$. All samples were run in duplicate. Cycling was performed at $95 \mathrm{C}^{\circ}$ for $5 \mathrm{~min}$ followed by a 45 -cycle amplification at $95^{\circ} \mathrm{C}$ for $10 \mathrm{~s}$, then at the annealing temperature defined previously for $15 \mathrm{~s}$ and at the temperature $72^{\circ} \mathrm{C}$ for $20 \mathrm{~s}$.

Results of the qPCR measurements were expressed as $\mathrm{Ct}$ values, where $\mathrm{Ct}$ is defined as the threshold cycle of PCR at which amplified product was $0.05 \%$ of normalized maximal signal. We used the comparative $\mathrm{Ct}$ method and computed the difference between the expression of the gene of interest and GAPDH expression in each cDNA sample (2- $\Delta \Delta \mathrm{Ct}$ method). Results are expressed as folds of expression compared to the mean values of expression in non-stressed control animals (Couch et al., adapted from Livak and Schmittgen 2001). 
Table 2. FLOATING IN THE MODIFIED SWIM TEST: ROLES OF SWIMMING, CONTEXT AND TIMING (Fig. 2)

\begin{tabular}{|c|c|c|c|}
\hline FACTOR & $\begin{array}{l}\text { Swimming } \\
\text { vs } \\
\text { context }\end{array}$ & Role of context & Role of timing \\
\hline Protocol & $\begin{array}{l}\text { Fig. 1 B } \\
\text { swimming is } \\
\text { replaced by a } \\
\text { context } \\
\text { exposure on } \\
\text { Day 2 }\end{array}$ & $\begin{array}{l}\text { Fig. } 1 \mathrm{C} \\
\text { no swimming, } \\
\text { no context exposure on } \\
\text { Day } 2\end{array}$ & $\begin{array}{l}\text { Fig. } 1 \mathrm{~F} \\
\text { swimming is on } \\
\text { Day } 3 \\
\text { instead of Day } 5\end{array}$ \\
\hline $\begin{array}{l}\text { Outcome: } \\
\text { floating on Day } 5\end{array}$ & $\uparrow$ & $=$ & $=$ \\
\hline
\end{tabular}

Table 2. Floating in the modified swim test: roles of swimming, context and timing. In series of experiments, the roles of swimming experience vs. context exposure, a reminder of a context of swimming, and a factor of timing of testing, in an increase of floating behaviour on Day 5 of the modified swim test, were dissected. Mice were exposed to the modified swim test with additional delayed testing on Day 5 or several variants of this protocol presented on Fig. 1. In comparison to the preceding session, the duration of floating behaviour on Day 5 was augmented $(\uparrow)$ or not altered $(=)$ in applied protocols of testing. 
Table 3. BRAIN mRNA GSK3B IN MODIFIED SWIM TEST: ROLES OF SWIMMING, CONTEXT AND TIMING (Fig. 3)

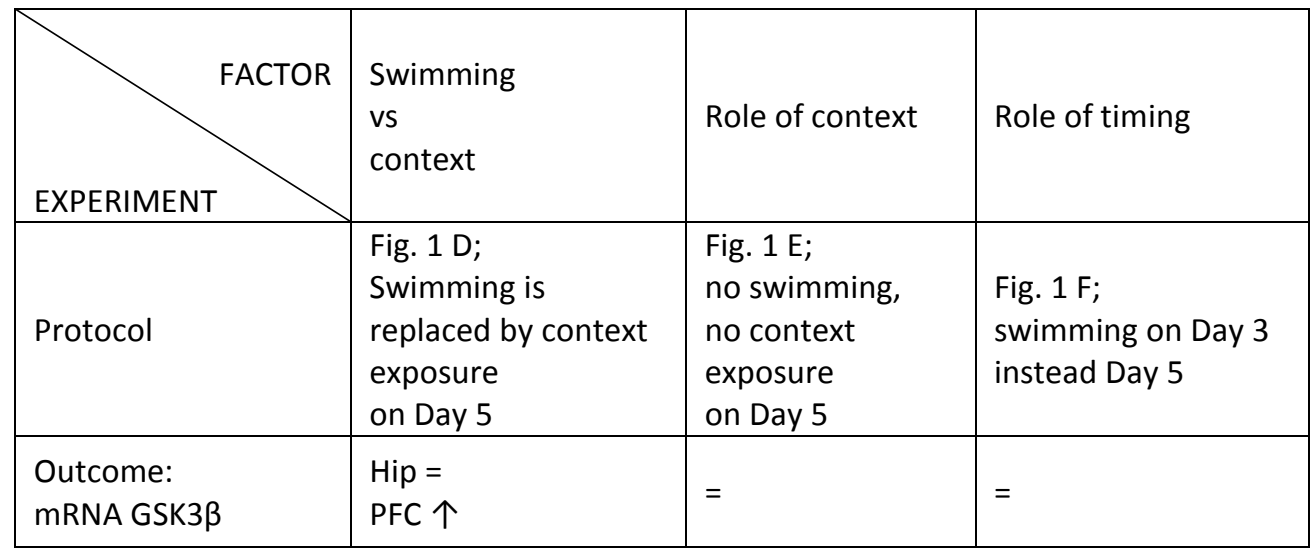

Table 3. mRNA GSK3 $\beta$ in the modified swim test: roles of swimming, context and timing. In series of experiments, the roles of swimming experience vs. context exposure, a reminder of a context of swimming, and a factor of timing of testing, in an increase of mRNA GSK3 $\beta$ on Day 5 of the modified swim test, were addressed. Mice were exposed to the modified swim test with additional delayed testing on Day 5 or several variants of this protocol presented on Fig. 1 . In comparison to the preceding session, the mRNA GSK3 $\beta$ level in the hippocampus (Hip) and prefrontal cortex (PFC) on Day 5 was augmented $(\uparrow)$ or not altered $(=)$ in applied protocols of testing. 
Table 4. A COMPARISON OF FLOATING AND GSK3B BRAIN ACTIVITES BETWEEN DAY 1-, DAY 2- AND DAY 5- (MODIFIED) SWIM TEST PROTOCOLS

\begin{tabular}{|c|c|c|c|c|c|}
\hline \multirow{2}{*}{$\begin{array}{l}\text { RARAMETER } \\
\text { TEST } \\
\text { SESSION }\end{array}$} & \multirow{2}{*}{ FLOATING } & \multicolumn{2}{|l|}{ mRNA GSK3 $\beta$} & \multicolumn{2}{|c|}{ pGSK3 $\beta$ / Total GSK3 $\beta$} \\
\hline & & Hip & PFC & Hip & PFC \\
\hline Day 1 & & vs $\operatorname{lnt}=$ & vs $\ln t=$ & vs $\operatorname{lnt}=$ & vs Int $=$ \\
\hline Day 2 & vs Day $1 \uparrow$ & $\begin{array}{l}\text { vs Int }= \\
\text { vs Day } 1=\end{array}$ & $\begin{array}{l}\text { vs Int }= \\
\text { vs Day } 1=\end{array}$ & $\begin{array}{l}\text { vs Int } \downarrow \\
\text { vs Day } 1 \downarrow\end{array}$ & $\begin{array}{l}\text { vs Int } \downarrow \\
\text { vs Day } 1 \\
\downarrow\end{array}$ \\
\hline Day 5 & $\begin{array}{l}\text { vs Day } 1 \uparrow \\
\text { vs Day } 2 \uparrow\end{array}$ & $\begin{array}{r}\text { vs Int LF } \uparrow, \\
H F \uparrow \\
\text { vs Day } 1 \mathrm{LF} \uparrow, \\
\mathrm{HF} \uparrow\end{array}$ & $\begin{array}{r}\text { vs Int } L F=, \\
H F \uparrow \\
\text { vs Day } 1 \mathrm{LF}= \\
\mathrm{HF} \uparrow\end{array}$ & $\begin{array}{r}\text { vs Int LF } \downarrow \\
H F \downarrow \\
\\
\text { vsDay } 1 F \downarrow \\
H F \downarrow\end{array}$ & $\begin{array}{r}\text { Vs Int } \\
\text { LF } \downarrow \\
\\
\text { vs Day1 } \\
\text { HF } \downarrow\end{array}$ \\
\hline
\end{tabular}

Table 4. A comparison of floating and GSK3 $\beta$ brain activities between Day 1-, Day 2- and Day 5- (modified) swim test protocols. Total duration of floating, brain GSK3 $\beta$ gene expression and protein levels were compared between the groups of mice sacrificed 10 min after testing at Day 1, Day 2 or delayed session on Day 5, in the swim test. mRNA GSK3 $\beta$ and a ratio pGSK3 $\beta$ / Total GSK3 $\beta$ were evaluated in the hippocampus (Hip) and prefrontal cortex (PFC) of subgroups of mice defined as Low Floaters (LF, see manuscript text) and High Floaters (HF, see manuscript text) post-testing on Day 2 and Day 5, and were compared to the values determined in intact mice (Int, see manuscript text) and in mice sacrificed at Day 1 of testing. A scheme of testing / sacrifice for each protocol is presented on Fig. 1A. Evaluated behavioural and molecular measures were augmented $(\uparrow)$, decreased $(\downarrow)$ or not altered $(=)$ in above-described comparisons. 
Table 5. EFFECTS OF IMIPRAMINE ON FLOATING AND GSK3B ACTIVITIES IN THE MODIFIED FORCED SWIM TEST

\begin{tabular}{|l|l|l|l|l|l|}
\hline \multirow{2}{*}{ RARAMETER } & \multirow{2}{*}{ FLOATING } & \multicolumn{2}{l|}{ mRNA GSK3 $\beta$} & \multicolumn{2}{l|}{ pGSK3 $\beta$ / Total GSK3 $\beta$} \\
\cline { 3 - 6 } & & Hip & PFC & Hip & PFC \\
\hline $\begin{array}{l}\text { DREATMENT } 5 \\
\text { No Drug }\end{array}$ & $\begin{array}{l}\text { vs Day 1 } \uparrow \\
\text { vs Day 2 } \uparrow\end{array}$ & $\begin{array}{l}\text { vs Int } \uparrow \\
\text { vs Day 1 } \uparrow \\
\text { vs Day 2 } \uparrow\end{array}$ & vs Int $=$ & vs Int $\downarrow$ & vs Int $\downarrow$ \\
\hline $\begin{array}{l}\text { Day 5 } \\
\text { Imi }\end{array}$ & $\begin{array}{l}\text { vs Day 1 } \uparrow \\
\text { vs Day 2 }=\end{array}$ & vs Int $\downarrow$ & vs Int $\uparrow$ & vs Int $=$ & vs Int $\downarrow$ \\
\hline
\end{tabular}

Table 5. Effects of imipramine on floating and GSK3 $\beta$ activities in the modified forced swim test. Total duration of floating, brain GSK3 $\beta$ gene expression and protein levels were compared between the groups of mice sacrificed 10 min after testing at delayed session on Day 5 of the swim test, which either received no drug or were treated with a low dose of imipramine. mRNA GSK3 $\beta$ and a ratio pGSK3 $\beta$ / Total GSK3 $\beta$ were evaluated in the hippocampus (Hip) and prefrontal cortex (PFC) of these groups and were compared to the values determined in intact mice (Int, see manuscript text) and in animals sacrificed at Day 1 and Day 2 of testing. A scheme of testing / sacrifice for each protocol is presented on Fig. 1G. Evaluated behavioural and molecular measures were augmented $(\uparrow)$, decreased $(\downarrow)$ or not altered $(=)$ in above-described comparisons. 


\section{Chapter 5. Thiamine and its precursor benfotiamine ameliorate cognitive}

abilities and GSK3 $\beta$-related depressive- and anxiety-like behaviours in mice

Nataliia Markova ${ }^{1,2,3,4^{*}}$, Nataliia Bazhenova ${ }^{1,3,4^{*}}$, Daniel C. Anthony ${ }^{5 \#}$, Julie Vignisse $^{1,6}$, Andrey Svistunov ${ }^{4}$, Klaus-Peter Lesch ${ }^{1,4,7}$, Lucien Bettendorff ${ }^{1,6 \S}$ and Tatyana Strekalova ${ }^{1,4 \S \#}$

${ }^{1}$ EURON - European Graduate School for Neuroscience, School for Mental Health and Neuroscience, Maastricht University, Universiteitssingel 40, NL 6229 ER Maastricht, Netherlands; ${ }^{2}$ Institute of Physiologically Active Compounds, Russian Academy of Sciences, Severnii proezd, 1, Chernogolovka, Russia, 142432; ${ }^{3}$ Institute of General Pathology and Pathophysiology, Baltiiskaya str, 8, Moscow, Russia, 125315; ${ }^{4}$ I.M. Sechenov First Moscow Medical University, Moscow, Russia; ${ }^{5}$ Department of Pharmacology, Oxford University, Mansfield Road, OX1 3QT, Oxford, UK; ${ }^{6}$ GIGA-Neurosciences, University of Liege, 4000 Liege Belgium; ${ }^{7}$ Division of Molecular Psychiatry, Laboratory of Translational Neuroscience, Department of Psychiatry, Psychosomatics and Psychotherapy, University of Wuerzburg, Fuechsleinstrasse 15, 97080, Wuerzburg, Germany

*§these authors contributed to this work equally

\# Joint Corresponding authors

Prof. Daniel C. Anthony, E-mail: daniel.anthony@pharm.ox.ac.uk

Dr. Tatyana Strekalova, M.D., E-mail: t.strekalova@maastrichtuniversity.nl

Markova, N., Bazhenova, N., Anthony, D.C., Vignisse, J., Svistunov, A., Lesch, K.-P., Bettendorff, L., Strekalova, T., 2016. Thiamine and benfotiamine improve cognition and ameliorate GSK3B-associated stressinduced behaviours in mice. Prog. Neuro-Psychopharmacology Biol. Psychiatry. 75, 148-156. 


\section{Abstract}

Brain thiamine deficiency has been implicated in the development of dementia and pathogenesis of depression. Among many reported functions, thiamine is suggested to control the phosphorylation of glycogen synthase kinase (GSK)-3 $\beta$, which regulates neuronal plasticity and is a marker of distress/depressive state. Here, we administered thiamine or, the more bioavailable precursor, benfotiamine at $200 \mathrm{mg} / \mathrm{kg} /$ day for 2 weeks, to study whether treatment might affect depressive-like behaviour/stress response and contextual learning in mouse paradigms that are sensitive to GSK3 $\beta$ dependent mechanisms. C57BL/6J mice were tested in models of contextual conditioning and extinction, a 5-day rat exposure stress test, and a modified swim test with repeated testing. Imipramine $(7.5 \mathrm{mg} / \mathrm{kg} / \mathrm{day})$ was administered as a positive control in the two latter experiments. Like imipramine, both compounds inhibited upregulation of GSK3 $\beta$ induced by stress or repeated swimming, and ameliorated floating scores and stressinduced behavioural changes in anxiety and exploration. Thiamine and benfotiamine improved learning and extinction of contextual fear and the acquisition of the step-down avoidance task. Together, our data indicate that thiamine and benfotiamine have pro-cognitive and antidepressant/antistress effects, which may be attributable to the differential modulation of GSK3 $\beta$ activity during adaptive versus maladaptive processes.

Keywords: thiamine, benfotiamine, Glycogene-Synthase-Kinase-3-beta (GSK3 $\beta$ ), plasticity, anxiety, exploration, predation stress, swim test

\section{Introduction}

Thiamine (vitamin B1) is a pivotal regulator of mitochondrial function and metabolism. Thiamine is the precursor of thiamine diphosphate, and acts as a cofactor for several rate limiting enzymes in the Krebs cycle and the pentose phosphate pathway (Bettendorff et al., 2014). Compromised thiamine-dependent processes result in mitochondrial dysfunction and downstream oxidative stress, excitotoxicity, inflammatory changes, 
decreased neurogenesis and blood-brain barrier disruption (Abdou and Hazell, 2015). In addition, thiamine plays a role in the structural stabilization of neuronal membranes via non-enzymatic mechanisms (Mkrtchyan et al., 2015). Deficiencies in thiamine metabolism accompany chronic alcohol exposure and diabetes, and lead to neurodegenerative and depressive symptoms (Benton and Donohoe, 1999; Abdou and Hazell, 2015). Strikingly, the addition of a daily dose of $300 \mathrm{mg}$ thiamine compared to 6 weeks of standard fluoxetine treatment was shown to improve Hamilton Depression Rating Scores in patients with major depression in a recent randomized double-blind placebo-controlled clinical trial (Ghaleiha et al., 2016).

A number of thiamine precursors that exhibit improved bioavailability have been described, including benfotiamine, which has been investigated in animal models of Alzheimer's disease and other conditions to explore its efficacy (Balakumar et al., 2010; Portari et al., 2013). Both thiamine and benfotiamine suppress oxidative stress, inflammation and apoptosis (Abdou and Hazell, 2015). An 8-week treatment of benfotiamine reduced learning deficiencies (measured in the Morris Water maze), and decreased cortical amyloid plaque formation and phosphorylated tau levels in amyloid precursor protein/presenelin-1 overexpressing mice in a dose-dependent manner (Pan et al., 2010). Studies using supplementary thiamine in patients with Alzheimer's disease failed to reveal any clinical efficacy, but this was ascribed to limited intestinal absorption in these patients (Rodriguez-Martin et al., 2001).

The mode of action of thiamine in depressive illness and neurodegenerative disease is unclear. The anti-thiamine compound pyrithiamine and dietinduced thiamine deficiency decrease the phosphorylation rates of Glycogen

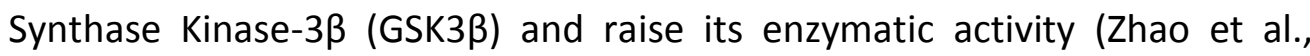
2014). GSK3 $\beta$ activation has become a well-recognised marker of distress and depression (Beurel, 2015). Benfotiamine decreases GSK3 $\beta$ activity in vitro by increasing the phosphorylation of GSK3 $\beta$ at serine 9 (p9SGSK3 $\beta$ ), which renders the enzyme inactive (Sun et al., 2012). Oral dosing of mice with thiamine or benfotiamine for 2 weeks increased brain thiamine levels and, for benfotiamine, an 8-week-dosing regimen reduced GSK3 $\beta$ activities 
via increasing the proportion of P9SGSK3 $\beta$ in amyloid precursor protein/presenelin-1 overexpressing mice (Pan et al., 2010). Thus while downregulation of GSK3 $\beta$ has been implicated as the mediator of thiaminedependent mechanisms in the CNS, to date, there has been no direct in vivo evidence for its role under normal conditions.

Inactivation of GSK3 $\beta$ results in learning and long-term potentiation deficiencies (Sintoni et al., 2013; Jurado-Arjona et al., 2016), while thiaminemediated functions are important for brain plasticity (Bettendorff, 2014). Low GSK3 $\beta$ activity has been linked to anti-depressant changes (KaidanovichBeilin and Woodgett, 2011; Li et al., 2014), which, as mentioned above, were recently reported to result from chronic thiamine administration (Ghaleiha et al., 2016). Given these seemingly opposing effects of GSK3 $\beta$ inactivation on cognition and depression/stress-related mechanisms, we studied whether administration of thiamine or benfotiamine $(200 \mathrm{mg} / \mathrm{kg} / \mathrm{day}$, p.o.) could alter GSK3 $\beta$-dependent tasks such as the acquisition of memory, depressive-like behaviour and the stress response. Thiamine- or benfotiamine-treated C57BL/6J mice were studied in the modified swim test, where depressive-like behaviour has previously been shown to correlate with over-expression of brain GSK3 $\beta$ in the brain (Strekalova et al., 2016) and a 5-day rat exposure stress paradigm, which suppresses hippocampal neurogenesis and increases anxiety scores in mice (Strekalova et al., 2015b). Finally, contextual fear learning and its extinction, in which GSK3 $\beta$ mechanisms are well-known to have a pivotal role (Chew et al., 2015), are also investigated here (Vignisse et al., 2011, 2014).

\section{Methods}

\section{Animals}

3.5-month-old male C57BL/6J mice used in the study were obtained from the Gulbenkian Institute of Science, Oeiras, Portugal. 14 days before the start of the behavioural tests, mice were housed individually for acclimatization to a new facility, under a reversed 12-h light-dark cycle (lights on: 21:00 h) with food and water ad libitum, under constant controlled laboratory conditions $\left(22 \pm 1^{\circ} \mathrm{C}, 55 \%\right.$ humidity). All experiments 
were carried out in accordance with the European Committees Council Directives with the European Union's Directive 86/609/EEC and Council Directive 93/119/EC, and had been approved by the ethic committee for animal research of Maastricht University CPV and by General Directory of Ethical Committee of the New Lisbon University.

\section{Study design}

Mice were administered with thiamine, benfotiamine or imipramine via drinking water for two weeks and subjected to the modified swim test, predation stress, or a set of memory tests (Fig. 1A-C; group sizes are given in Figs.2-4) (see below for details). For the modified swim test, sessions of 6 minutes in duration were performed on Days 1 and 2 and 5, and animals were culled 10 minutes post-test, and simultaneously with naïve mice that were not exposed to the swim test or administration of substances. The hippocampus and prefrontal cortex were dissected and prepared for GSK3 $\beta$ RT-PCR analysis and an ELISA assay was used to determine total and proportion of 9-Ser-phosphorylation in the hippocampus (Fig. 1A). All procedures were carried out after at least 1-hour acclimatization time to experimental room.

\section{Behaviour}

Swim test. In the modified swim test model, mice were exposed to a two-day swimming protocol (day 1 and day 2); they were then tested on again on day 5 (Markova et al., 2013, 2014; Strekalova et al., 2016). In each immersion, naïve mice or those dosed with imipramine, thiamine or benfotiamine were placed for $6 \mathrm{~min}$ in a transparent cylinder $(\varnothing 17 \mathrm{~cm})$ filled with water $\left(+23^{\circ} \mathrm{C}\right.$, water height $13 \mathrm{~cm}$, height of cylinder $20 \mathrm{~cm}$, under subdued lighting). Floating behaviour, defined by the absence of any directed movement of the head or body, was manually scored using previously established criteria using Noldus EthoVision XT 8.5 (Noldus Information Technology, Wageningen, The Netherlands) and CleverSys (CleverSys, Reston, VA, USA) as described elsewhere (Malatynska et al., 2012). The total time spent floating was calculated for the entire duration of the test. 
Elevated O-maze. The apparatus (Technosmart, Rome, Italy) consisted of a circular path (runway width $5.5 \mathrm{~cm}$, diameter $46 \mathrm{~cm}$ ) that was placed $45 \mathrm{~cm}$ above the floor. The two opposing arms were protected by walls (closed area, height $10 \mathrm{~cm}$ ), and the illumination strength was 25 Lux. The apparatus was placed on a dark surface in order to maintain control over lighting conditions during testing. At the start, mice were placed in one of the closed-arm area of the apparatus, behaviour was video recorded and assessed for a 5-minute observation period as described elsewhere (Vignisse et al., 2011, Strekalova et al., 2015a). The latency to the first exit into the open compartments of the maze and the number of exits to the open arms were recorded.

Novel cage test. The novel cage test was performed to assess vertical exploratory activity in a new environment (Strekalova and Steinbusch, 2010; Couch et al., 2013). Mice were introduced into a standard plastic cage $(21 \times 21 \times 15 \mathrm{~cm})$ filled with fresh sawdust. The number of exploratory rears each minute was counted under red light for a 5-min period.

Step-down passive avoidance test. The step-down apparatus (Technosmart, Rome, Italy) consisted of a transparent plastic cubicle $(25 \mathrm{~cm} \times 25 \mathrm{~cm} \times 50$ $\mathrm{cm}$ ) with a stainless-steel grid floor (33 rods $2 \mathrm{~mm}$ in diameter) onto which a square wooden platform $(7 \mathrm{~cm} \times 7 \mathrm{~cm} \times 1.5 \mathrm{~cm})$ was placed. A shocker was used to deliver an alternating electric current $(A C, 50 \mathrm{~Hz}$, Evolocus, Terrytown, NY, USA). In this paradigm, animals were trained not to step down from a platform onto a grid floor to avoid an electric shock. During the training session, mice were placed onto the platform inside a transparent cylinder for $30 \mathrm{~s}$ to prevent them from immediately stepping down. After removal of the cylinder, the time until the animal left the platform with all four paws was measured as baseline latency of step down. Immediately after step down, mice received a single electric foot-shock (0.5 mA, $2 \mathrm{~s})$ and returned to their home cages. During the recall trial session, animals faced the same context as in the training session. The latency to the animal stepping-down was recorded $1 \mathrm{~h}$ and $24 \mathrm{~h}$ post-training, latency of step down with all four paws was measured until 180s elapsed. According to previously validated criteria for the acquisition of the step down avoidance task, an 
increase of latencies measured in animals during a recall session are taken as a sign of long-term learning (Strekalova et al., 2002, Vignisse et al., 2011, 2014). A percentage of "good learners", i.e., mice in whom this measure was $>30$ s at $24 \mathrm{~h}$ post-training, was calculated (Vignisse et al., 2011) (Fig. 1C).

Fear conditioning learning and extinction. In a separate study, mice were trained with a $2 \mathrm{~s}$ foot-shock of "weak" $(0.5 \mathrm{~mA}, 50 \mathrm{~Hz})$ or "strong" $(0.7 \mathrm{~mA}$, $50 \mathrm{~Hz}$ ) intensity in the fear conditioning paradigm, which was delivered after a 2-min acclimatization period using a shocker (Evolocus, Terrytown, NY, USA). The apparatus (Open Science, Russia and Technosmart, Rome, Italy) consisted of a transparent plastic cubicle $(25 \times 25 \times 50 \mathrm{~cm})$ with a stainlesssteel grid floor (33 rods $/ 2 \mathrm{~mm}$ in diameter). After delivery of the current, the mouse was immediately placed back into the home cage. Twenty-four hours later, freezing behaviour was scored in a 180-s recall of extinction session. The occurrence of freezing behaviour was assessed every 10s, and each 10-s period was assigned to a freezing or non-freezing period, and the percentage of time spent freezing was calculated. Animals subjected to "strong" shocks were exposed to a memory extinguishing procedure immediately after a recall session (Strekalova et al., 2003, Vignisse et al., 2014). Therefore, mice were left for another seven minutes in the apparatus, so the total procedure of memory extinction was 10-min long. During this period, no foot shock was applied, and animals were free to explore the apparatus. Twenty-four hours later, freezing behaviour was scored again in a 180-s recall of extinction session as in the previous trial and percentage of time spent in freezing was calculated.

Predator stress. A previously established protocol was used (Strekalova et al., 2015a). Mice were introduced into cylindrical containers, which were placed into a rat home cage for $15 \mathrm{~h}$ (over-night, from $18 \mathrm{~h} 00$ to 9h00) for 5 nights. The containers were made from customized transparent plastic, size $15 \mathrm{~cm} \times \varnothing 8 \mathrm{~cm}$, with holes in covers $(\varnothing<0.5 \mathrm{~cm})$, which ensured protection of the mouse from the rat, but allowed visual and odour contact. Afterwards, animals were scored for exploratory rears in the novel cage test, latency and number of exit(s) to the open arms of the O-maze (Vignisse et al., 2011; see also below for details); they were killed $5 \mathrm{~h}$ post-stress and the 
hippocampus and prefrontal cortex were dissected for GSK3 $\beta$ RT-PCR assay (see below), simultaneously with naïve controls that were not exposed to any manipulations (Fig. 1B).

Brain dissection, RNA extraction and RT-PCR.

Mice were killed by cervical dislocation as it was described elsewhere (CostaNunes et al., 2014). The brain of each mouse was dissected and the prefrontal cortex and the hippocampus were isolated as previously reported (Couch et al., 2013; Strekalova et al., 2016) and stored at 80?C until later use. mRNA was extracted by using TRI Reagent (MRC, USA). First strand cDNA synthesis was performed using random primers and Superscript III transcriptase (Invitrogen, Darmstadt, Germany); $1 \mathrm{mcg}$ total RNA was converted into cDNA. Quantitative RT-PCR (qRT-PCR) for glycogen synthase kinase 3 beta (GSK3ß) gene and the housekeeping gene glyceraldehyde-3phosphate dehydrogenase (GAPDH) was performed using the SYBR Green master mix (Bio-Rad Labouratories, Philadelphia, USA) and the CFX96 Realtime System (Bio-Rad Labouratories, Philadelphia, USA). The sequences of the primers used are indicated in Supplementary Table 1 (see below). Data were normalized to GAPDH mRNA expression and calculated as relative-fold changes compared to control mice as described elsewhere (Couch et al., 2013; Strekalova et al., 2016). Results of qRT-PCR measurement were expressed as $\mathrm{Ct}$ values, where $\mathrm{Ct}$ is defined as the threshold cycle of PCR at which amplified product was $0.05 \%$ of a normalized maximal signal. We used the comparative $\mathrm{Ct}$ method and computed the difference between the expression of the gene of interest and GAPDH expression in each cDNA sample (2- $\Delta \Delta \mathrm{Ct}$ method). Data are given as expression-folds compared to the mean expression values in non-stressed control mice (Couch et al., 2013).

\section{ELISA assays of total and phosphorylated forms of GSK3B}

Total GSK3 $\beta$ and p9SGSK3 $\beta$ were measured by ELISA, and their ratio was calculated as described elsewhere (Strekalova et al., 2016). The hippocampus were homogenized in buffer containing $10 \mathrm{mM}$ Tris (pH 7.4), $100 \mathrm{mM} \mathrm{NaCl}, 1 \mathrm{mM}$ EDTA, 1 mM EGTA, 1 mM NaF, 20 mM Na4P2O7, 10\% 
glycerol, 2 mM Na3VO4. A Protease inhibitor cocktail (Sigma-Aldrich, St. Louis, MO, USA) was added immediately prior to homogenization. The [pS9]GSK3 $\beta$ ELISA kit and [total]-GSK3 $\beta$ ELISA kit (Invitrogen Corporation, Carlsbad, CA, USA) were used for quantification of the level of GSK3 $\beta$ protein phosphorylated at serine residue 9 and total level of GSK3 $\beta$. All procedures were performed according the manufacturer's instructions; the absorbance was measured at $450 \mathrm{~nm}$ using a plate reader (Wallac 1420 VICTOR, Waltham, MA, USA). The results were normalized to total protein level in tissues homogenates. Protein concentrations were determined by the biuret assay using bovine serum albumin as a standard.

\section{Drug delivery}

Experimental solutions replaced normal drinking water. Thiamine, benfotiamine and imipramine were obtained from Sigma-Aldrich, St. Louis, MO, USA. All agents were dissolved in tap water and were changed every 4-5 days (Strekalova et al., 2006, 2015b). The solutions of benfotiamine and thiamine were adjusted to $\mathrm{pH}$ 7.0, drinking behaviour of mice was monitored by evaluating a 24-h liquid intake during the first three days of dosing. The dose of imipramine dose $(7.5 \mathrm{mg} / \mathrm{kg} / \mathrm{day})$, was based on previous results showing that this dose had no effect on general locomotor behaviour (Strekalova et al., 2015b; Costa-Nunes et al., 2015).

\section{Statistical analysis}

Data were analysed with GraphPadPrism v.5.0 for Windows (San Diego, CA, USA) using one-way ANOVA and Tukey's post-hoc test unless otherwise stated. Repeated measures (RM) ANOVA or Fisher's exact test were also employed where appropriate. The level of confidence was set at $95 \%$ ( $p<$ $0.05)$. Data are given as mean \pm SEM. 


\section{Results}

Thiamine and benfotiamine inhibit floating behaviour and brain GSK3B in the modified swim test

Statistical values for all results are presented in the Supplementary Tables 211. There was no difference in floating duration on day 1 and day $2(p>0.05$, one-way ANOVA, Supplementary Table 2A). Vehicle-treated mice displayed a significant increase in floating time from day 2 to day $5(p<0.05, R M$ ANOVA). This increase was not observed in any of the pharmacologically treated groups ( $p>0.05$ for each, RM ANOVA; Fig. 2A, Supplementary Table $2 B)$. Thus, the administration of thiamine and benfotiamine, as did imipramine, prevented behavioural changes in the modified swim depression paradigm. The swim test generated differences in GSK3 $\beta$ mRNA expression in the hippocampus and prefrontal cortex (main effect: $p<0.05$, one-way ANOVA, Supplementary Table 3). Compared to naïve mice, GSK3 $\beta$ mRNA was significantly elevated in vehicle-treated mice $(p<0.05$, Tukey test; Fig. 2B, C), but not in imipramine-treated mice, nor in the thiamine and benfotiamine groups $(p<0.05)$. Hippocampal GSK3 $\beta$, p9SGSK3 $\beta$, as measured by ELISA, and their ratio was also found to be significantly affected by treatment (main effect: $p<0.05$ ). Vehicle-treated mice showed an increase in total GSK3 $\beta$, reduced p9SGSK3 $\beta$ and a reduced p9SGSK3 $\beta /$ totalGSK3 $\beta$ ratio compared to naïve animals $(p<0.05$, Tukey test; Supplementary Fig. 1 and Table 4). Imipramine-treated mice, as well as thiamine- or benfotiamine-treated animals showed no such change ( $p$ > $0.05)$ and gave rise to levels that were similar to those in animals treated with imipramine.

Thiamine and benfotiamine ameliorate stress-induced behaviour and GSK3B expression after a predation stress

There was a significant between-group difference in exploratory rears in the novel cage test for the experimental groups ( $p<0.05$, one-way ANOVA, Supplementary Table 5). In comparison with naïve mice, rearing behaviour was significantly decreased in stressed vehicle-treated mice $(p<0.05$, Tukey test; Fig. 3A), but not in stressed imipramine-, thiamine- or benfotiamine- 
treated groups ( $p>0.05$ ) that displayed significantly higher exploration than stressed vehicle-treated animals $(p<0.05)$. In the elevated O-maze test, we found significant differences in the latency to exit the open arms and in the total number of exits ( $p<0.05$, one-way ANOVA; Supplementary Table 6$)$. In comparison with naïve animals, those in the stressed vehicle-treated group made significantly fewer exits in to the open arms and had a tendency to prolonged latency to exit ( $p<0.05$ and $p=0.07$, Tukey test; respectively; Fig. $3 \mathrm{~B}, \mathrm{C})$. This stress-induced change was not observed in the imipraminetreated group, nor in the thiamine- or benfotiamine-treated mice stressed mice $(p>0.05)$.

ANOVA revealed significant differences between experimental groups in GSK3 $\beta$ mRNA in the prefrontal cortex, but not in the hippocampus $(p<0.05$ and $p>0.05$, respectively, Supplementary Table 7). Compared with naïve controls, stressed vehicle-treated mice displayed a non-significant change in GSK3 $\beta$ mRNA level in the hippocampus ( $p>0.05$, Tukey test; Fig. 3D) and a significant increase in levels in prefrontal cortex $(p<0.05$, Fig. 3E). Stressed animals treated with imipramine, or thiamine, or benfotiamine showed no such changes ( $p>0.05$ vs. naïve mice). Thiamine and benfotiamine, as for imipramine, prevented the stress-induced changes in exploratory behaviour, anxiety-like behaviour and over-expression of GSK3 $\beta$ in the brain.

\section{Effects of thiamine and benfotiamine on memory}

In the step-down test (Supplementary Tables 8,9), there were no differences in the baseline step-down latencies, measured prior training, between all experimental groups ( $p>0.05$, one-way ANOVA; data not shown), suggesting that normal scores of anxiety and locomotion are preserved in the test groups. Step-down latencies measured 1 and 24 hours post-training were significantly different across experimental groups ( $p<0.05$, one-way ANOVA). In comparison with vehicle-treated controls, there was a significant increase of step-down latency in thiamine- and benfotiamine-treated mice 1 $h$ post-training, and in thiamine-treated groups $24 \mathrm{~h}$ post-training $(p<0.05$, Tukey test; Fig. 4A). A higher percentage of animals were grouped as "good learners" among the thiamine- and benfotiamine-treated as compared to non-treated mice ( $p<0.05$, Fisher test; Fig. 4B). The number of rears in the 
novel cage did not differ between experimental groups ( $p>0.05$, Tukey test; Fig. 4C, Supplementary Table 10), further suggesting a lack of effects of applied treatments on locomotor activity.

There was a significant difference in freezing behaviour observed during a recall session between the groups trained under "weak", but not "strong" conditioning ( $p<0.05$ and $p>0.05$, one-way ANOVA, Supplementary Table 11). The thiamine- and benfotiamine-treated mice groups spent significantly longer time freezing after a "weak" training than vehicle-treated mice $(p<$ 0.05, Tukey test; Fig. 4D); no such difference was found in a "strong" training protocol ( $p>0.05$; Fig. $4 \mathrm{E}$ ). At the recall of extinction, there was a significant between-group difference in freezing behaviour ( $<0.05$, one-way ANOVA). As compared with vehicle-treated controls, thiamine- and benfotiaminetreated mice spent significantly less time freezing, demonstrating effective fear extinction ( $p<0.05$, Tukey test; Fig. 4F). Together, these data suggest improved short- and long-term contextual memory and its extinction in thiamine- and benfotiamine-treated animals compared with untreated animals, and a lack of non-specific locomotor changes.

\section{Discussion}

Our study has demonstrated, for the first time that the two-week treatment of mice with thiamine or benfotiamine had beneficial effects in classical paradigms of learning and depression/stress-related behaviours. Our results also provide the first evidence that these treatments are associated with decreased GSK3 $\beta$ activity in normal mice. Previously reported in vitro and in vivo studies with benfothiamine have revealed its inhibitory effects on GSK3 $\beta$ activities in pathological conditions, such as models of Alzheimers pathology (Pan et al., 2010; Sun et al., 2012), but whether the beneficial effects might be induced in non-pathological states where hitherto unknown.

The treatment of mice with thiamine or benfotiamine, as for imipramine, inhibited changes induced by forced swimming or predation stress. Thiamine deficiency is known to induce floating behaviour in mice (Nakagawasai et al., 2007). A 30-day-long dosing regimen with thiamine in Wistar rats that were 
subjected to a chronic immobilization stress rescued normal learning in the T-maze, decreased freezing scores, increased locomotion in the open field, and reduced anxiety-like behaviours (Dief et al., 2015). The present study also supports the latest findings showing that thiamine supplementation has beneficial effects in depressed patients (Ghaleiha et al., 2016), and improves mood in healthy adults (Schmidt et al., 1991; Benton et al., 1997) and is in accord with evidence for a link between lowered thiamine level and stronger symptoms of depression of various forms (Bell et al., 1991, 1992; Pepersack et al., 1999; Zhang et al., 2013). However, there is no prior literature available on the anti-stress/anti-depressant effects of benfotiamine in animal models.

Behavioural changes provoked by the swim-test and the exposure to a predation stress are both accompanied by inhibition of GSK3 $\beta$ mRNA expression in the hippocampus and the prefrontal cortex. Elevated GSK3 $\beta$ mRNA and lowered p9SGSK3 $\beta /$ totalGSK3 $\beta$ ratio in the brain are established markers for depression-like and distress syndromes (Kaidanovich-Beilin and Woodgett, 2011; Zhou et al., 2012; Li et al., 2014). As for imipramine-treated mice, thiamine-treated or benfotiamine-treated rescued levels of GSK3 $\beta$ mRNA, p9SGSK3 $\beta$, totalGSK3 $\beta$ and p9SGSK3 $\beta /$ totalGSK3 $\beta$ ratio in the stressed animals and were no different to the values determined for naïve animals. In the modified swim test, dosing with either drug had no effect on behaviour on days 1 and 2, which was at a time when no changes in GSK3 $\beta$ mRNA were observed, but, on day 5 , when increases of both floating and GSK3 $\beta$ on gene and protein levels were reported (Strekalova et al., 2016) the drugs were effective. Together, this association argue that the effects of thiamine- and benfotiamine could be attributable to the downregulation of GSK3 $\beta$ activity.

As well as modifying stress and anxiety responses, the two-week treatment with thiamine or benfotiamine in naïve animals were shown to enhance contextual fear learning and its extinction, which, are known to require intact GSK3 $\beta$ function (Beurel, 2015; King, 2015). Our experiments showed that treatments with thiamine or benfotiamine are able to increase hippocampal-dependent memory in two classical tests for this form of 
learning that is generally in line with established views on the role of thiamine in neuronal plasticity and pro-cognitive effects of benfotiamine in an Alzheimer's disease model (Pan et al., 2010; AbBou and Hazell, 2015). To our knowledge, this is the first demonstration of such effects of these compounds in naïve mice. The present data suggest that moderate procognitive effects are associated with the administration of thiamine or benfotiamine, which improved fear conditioning after "weak" training. A lack of effect under "strong" training conditions is likely due to ceiling effect of the training, when physiologically adaptive learning cannot be enhanced any further. Similar difference in sensitivity of contextual learning during "weak" versus "strong" training to mnemotropic agents was shown in our previous studies to be correlated with outcome from "weak" and "strong" protocols for the induction of long-term potentiation paradigms, where the same factors were used (Strekalova et al., 2002).

A lack of group differences in baseline step down as well as in vertical activity and measures of horizontal locomotion in the True Scan open field (see Supplementary Figure 2) rule out the possibility that general changes in anxiety and locomotion in thiamine- or benfotiamine-treated mice might account for the differences and strongly argues for improved memory in these groups.

The outcomes in the learning and modified swim test are believed to share overlapping mechanisms, such as elements of contextual conditioning. In the modified forced swim model of depression, both behavioural outputs and GSK3 $\beta$ mRNA over-expression are triggered by contextual reminders of the testing procedure alone, such as a visual exposure of an animal to a swimming tank, without its experience of a swimming (Strekalova et al., 2016). As such, modified swimming test is considered as a model of inappropriately increased (maladaptive) contextual conditioning of adverse memories, which plays an important role in a pathophysiology of depression (Clark et al., 2009). It is generally viewed that contextual conditioning can play both adaptive and pathological roles. While learning of new environment is of adaptive value for an individual, enhanced retention of negative contextual associations can be an important element of a 
pathophysiology of depression, post-traumatic stress disorder and generalized anxiety (Diamond et al., 2004; Clark et al., 2009).

In the present study, thiamine and benfotiamine inhibited contextual conditioning dependent on depressive-like behavioural and molecular changes, while it improved acquisition and extinction of contextual memories of a new environment that can be considered as adaptive. Based on the current findings, it could be speculated that thiamine and benfotiamine modulate GSK3 $\beta$ functions and contextual conditioning in a manner that is dependent on whether the contextual conditioning is adaptive or maladaptive.

Other studies showed discordant changes in hippocampal plasticity and affective processes in mice with systemically altered GSK3 $\beta$ activity, either via genetic manipulations (Pardo et al., 2016), or chronic pharmacological treatments, such as valproate (Sintoni et al., 2013). For instance, GSK3 $\beta$ knock-in mice with increased activity of this molecule displayed heightened vulnerability to the learned helplessness model of depression-like behaviour and showed no enhancement, but an impairment of some but not all forms of memory. Such forms of learning, such as novel object recognition and spatial processing, and not temporal order memory, were disrupted in GSK3 $\beta$ knock-in mice. In rats, chronic dosing with valproate decreased baseline brain p9SGSK3 $\beta$ /totalGSK3 $\beta$ ratio, however, did not attenuate their response to novel stressors, while impaired contextual fear conditioning. It was suggested that these effects were due to altered dynamics of GSK3 $\beta$ phosphorylation at 9 Ser that was due to distinct mechanisms involved in two behavioural tests (Sintoni et al., 2013). Thus, an increase or a decrease of brain GSK3 $\beta$ activities induced by chronic systemic interventions do not ultimately lead to concordant changes in mood and cognition, which can be expected from general roles of GSK3 $\beta$ in these conditions. The functions and regulation of GSK3 $\beta$ functions are very complex and can be moderated via multiple mechanisms including post-translational modifications, substrate priming, cellular trafficking, protein complexes (Beurel, 2015). Hence, a number of GSK3 $\beta$ regulated processes may contribute to the changes reported here and to those reported in earlier studies. 
Here, systemically administrated thiamine and benfotiamine were shown to increase brain thiamine levels to a similar extent (Vignisse et al., 2016). This could explain the similarity in the effects of the compounds in our study, despite the low reported bioavailability characteristic of thiamine. Indeed, in current experiments, thiamine was used at a high dose, exceeding by more than 10-fold the concentrations applied in the studies that reported its administration to be behaviourally ineffective (Rodriguez-Martin et al., 2001). Single or two-week administration of thiamine at the dose $100 \mathrm{mg} / \mathrm{kg}$ did not elevate thiamine brain levels (Volvert et al., 2008). In line with our results, chronic administration of thiamine produced antidepressant effect in a clinic. It is of note that the doses used in the clinical study ( $300 \mathrm{mg} /$ day) was substantially lower than that used in the present work (Ghaleiha et al., 2016). Neither treatment affected thiamine diphosphate content (Volvert et al., 2008, Vignisse et al., 2016) suggesting that the effects reported here are mediated by coenzyme-independent mechanisms (Mkrtchyan et al., 2015). Hence, it can be suggested that both anti-stress / anti-depressant and procognitive effects of employed here treatments are underpinned by noncoenzyme functions of thiamine, such as an increase of brain thiamine levels associated with subsequent modulation of GSK3 $\beta$ functions.

Apart from GSK3 $\beta$-related mechanisms, other factors could also underlie the effects reported here, especially the pro-cognitive effects, such as the altered production and activity of reactive oxygen species, anti-inflammatory activities, or changes in BDNF levels affecting biosynthesis of monoamines, including serotonin (Nakagawasai et al., 2007; AbBou and Hazell, 2015; Bozic et al., 2015), resulting, as for instance, in reductions in spine density and complexity, that have been associated with depression, stress and learning (Duman and Duman, 2015). These would be interesting to explore in future studies given the impact of the treatments on normal animals though many of these other effects can also be related back to GSK3 $\beta$-mediated cascades (King, 2014; Beurel, 2015).

\section{Conclusion}

Our results demonstrate that administration of thiamine, or it precursor, decrease GSK3 $\beta$ activity in limbic structures under conditions of stress. The 
current study also provides the first evidence for antidepressant-like effects and pro-cognitive action of thiamine, or its precursor, in a pre-clinical assays. Remarkably, the precognitive actions were shown in naïve animals. Thus, our data support a view that thiamine can be an important physiological regulator of stress response, learning and brain GSK- $\beta$ and reveal the potential thiamine/thiamine precursors as antidepressants and cognitive enhancers.

\section{Highlights}

- Thiamine and benfotiamine generate antistress and antidepressantlike effects in mice;

- Both molecules prevent brain activation of GSK3- $\beta$ in a model of depression;

- $\quad$ Each also prevent stress-induced increases in anxiety and GSK3- $\beta$ expression;

- $\quad$ Thiamine and benfotiamine enhance contextual memory and extinction in naïve mice;

- $\quad$ The effects on stress and learning are likely to be mediated via distinct mechanisms.

\section{Acknowledgements}

The authors' work reported here was supported by the European Community EC: AGGRESSOTYPE FP7/No.602805, "5-100" Russian Academic Excellence Project, Lucien Bettendorff is Research Director of the Funds for Scientific Research (F.R.S.-FNRS), Belgium.

\section{References}

[1]. Abdou, E., Hazell, A.S., 2015. Thiamine deficiency: an update of pathophysiologic mechanisms and future therapeutic considerations. Neurochem. Res. 40, 353-361.

[2]. Balakumar, P., Rohilla, A., Krishan, P., Solairaj, P., Thangathirupathi, A., 2010. The multifaceted therapeutic potential of benfotiamine. Pharmacol. Res. 61, 482-488. 
[3]. Bell, I.R., Edman, J.S., Morrow, F.D., Marby, D.W., Mirages, S., Perrone, G., Kayne, H.L., Cole, J.O., 1991. B complex vitamin patterns in geriatric and young adult inpatients with major depression. J Am Geriatr Soc. 39, 252-257.

[4]. Bell, I.R., Edman, J.S., Morrow, F.D., Marby, D.W., Perrone, G., Kayne, H.L, Greenwald, M., Cole, J.O., 1992. Vitamin B1, B2, a Vitamin B1, B2, and B6 augmentation tricyclic antidepressant treatment in geriatric depression with cognitive dysfunction. J Am Coll Nutr. 11, 159-163.

[5]. Benton, D., Griffiths, R., Haller, J., 1997. Thiamine supplementation mood and cognitive functioning. Psychopharm. 129, 66-71.

[6]. Benton, D., Donohoe, R.T., 1999. The effects of nutrients on mood. Public Health Nutr. 2(3a), 403-409.

[7]. Bettendorff L., Lakaye B., Kohn G., Wins P. 2014. Thiamine triphosphate: a ubiquitous molecule in search of a physiological role. Metab Brain Dis. 29, 1069-1082.

[8]. Bettendorff, L., 2014. Thiamine triphosphate: a ubiquitous molecule in search of a physiological role, in: Zempleni, J., Suttie, J.W., Gregory, J.F., Stover, P.J. (Eds.), Handbook of Vitamins, Chapter 7, fifth ed. CRC Press, Taylor \& Francis Group 2014, Boca Raton, pp. 267-323.

[9]. Beurel, E., Grieco, S.F., Jope, R.S., 2015. Glycogen synthase kinase-3 (GSK3): regulation, actions, and diseases. Pharmacol. Ther. 148, 114-131.

[10]. Bozic, I., Savic, D., Laketa, D., Bjelobaba, I., Milenkovic, I., Pekovic, S., Nedeljkovic, N., Lavrnja, I., 2015. Benfotiamine attenuates inflammatory response in LPS stimulated BV-2 microglia. PLoS One. 10, e0118372.

[11]. Chew, B., Ryu, J.R., Ng, T., Ma, D., Dasgupta, A., Neo, S.H., Zhao, J., Zhong, Z., Bichler, Z., Sajikumar, S., Goh, E.L., 2015. Lentiviral silencing of GSK3 $\beta$ in adult dentate gyrus impairs contextual fear memory and synaptic plasticity. Front. Behav. Neurosci. 9, 158.

[12]. Clark, L., Chamberlain, S.R., Sahakian, B.J., 2009. Neurocognitive Mechanisms in Depression: Implications for Treatment, Annu. Rev. Neurosci. 32, 57-74.

[13]. Costa-Nunes, J., Zubareva, O., Araújo-Correia, M., Valença, A., Schroeter, C.A., Pawluski, J.L., Vignisse, J., Steinbusch, H., Hermes, D., Phillipines, M., Steinbusch, H.M.W., Strekalova, T., 2015. Altered emotionality, hippocampus-dependent performance and expression of 
NMDA receptor subunit mRNAs in chronically stressed mice. Stress 17,108 116.

[14]. Costa-Nunes, J.P., Cline, B.H., Araújo-Correia, M., Valença, M., Markova, N., Dolgov, O., Kubatiev, A., Yeritsyan, N., Steinbusch, H.W., Strekalova, T., 2015. Animal models of depression and drug delivery with food as an effective dosing method: evidences from studies with celecoxib and dicholine succinate. Biomed. Res. Int. 2015, 596126.

[15]. Couch, Y., Anthony, D.C., Dolgov, O., Revischin, A, Festoff, B., Santos, A.I., Steinbusch, H.W., Strekalova, T., 2013. Microglial activation, increased TNF and SERT expression in the prefrontal cortex define stress-altered behaviour in mice susceptible to anhedonia. Brain. Behav. Immun. 29, 136146.

[16]. Diamond, D.M., Park, C.R., Woodson, J.C., 2004. Stress generates emotional memories and retrograde amnesia by inducing an endogenous form of hippocampal LTP. Hippocampus. 14, 281-291.

[17]. Dief, A., M., Samy, D, I., Dowedar, F., 2015. Impact of exercise and vitamin B1 intake hippocampal brain-derived neurotrophic factor and spatial memory performance in a rat model of stress. J. Nutr. Sci. Vitaminol. (Tokyo). 61, 1-7.

[18]. Duman, C.H., Duman, R.S. 2015. Spine synapse remodeling in the pathophysiology and treatment of depression. Neurosci Lett. 60, 20-29.

[19]. Ghaleiha, A., Davari, H., Jahangard, L., Haghighi, M., Ahmadpanah, M., Seifrabie, M.A., Bajoghli, H., Holsboer-Trachsler, E., Brand, S., 2016. Adjuvant thiamine improved standard treatment in patients with major depressive disorder: results from a randomized, double-blind, and placebocontrolled clinical trial. Eur. Arch. Psychiatry. Clin. Neurosci. Epub ahead of print.

[20]. Jurado-Arjona, J., Llorens-Martín, M., Avila, J., Hernández, F., 2016. GSK3 $\beta$ overexpression in dentate gyrus neural precursor cells expands the progenitor pool and enhances memory skills. J. Biol. Chem. 291, 8199-8213.

[21]. Kaidanovich-Beilin, O., Woodgett, J.R., 2011. GSK-3: Functional insights from cell biology and animal models. Front. Mol. Neurosci. 16, 40.

[22]. King, M.K., Pardo, M., Cheng, Y., Downey, K., Jope, R.S., Beurel, E., 2014. Glycogen synthase kinase-3 inhibitors: Rescuers of cognitive impairments. Pharmacol. Ther. 141, 1-12. 
[23]. Li, G., Liu, T., Kong, X., Wang, L., Jin, X., 2014. Hippocampal glycogen synthase kinase $3 \beta$ is critical for the antidepressant effect of cyclindependent kinase 5 inhibitor in rats. J. Mol. Neurosci. 54, 92-9.

[24]. Malatynska, E., Steinbusch, H.W., Redkozubova, O., Bolkunov, A., Kubatiev, A., Yeritsyan, N.B., Vignisse, J., Bachurin, S., Strekalova, T., 2012. Anhedonic-like traits and lack of affective deficits in 18-month-old C57BL/6 mice: Implications for modeling elderly depression. Exp. Gerontol. 47, 552564.

[25]. Markova, N., Shevtsova, E., Bachurin, S., Steinbusch, H.M.W., Strekalova, T., 2013. Individual susceptibility to depressive-like traits in two mouse models of depression and hippocampal levels of GSK3 $\beta$. The abstract book of The 38th FEBS Congress, Saint Petersburg, pp. 219-220.

[26]. Markova, N., Shevtsova, E., Vignisse, J., Zubareva, O., Trofimov, A., Steinbusch, H., Bachurin, S., Bettendorff, L., Strekalova, T., 2014. Thiamine and benfotiamine improve memory, decrease depressive-like behaviour and reduce brain expression of GSK3 $\beta$ in mice. The abstract book of The 8th International Conference on Thiamine: From Catalysis to Pathology, Liege, pp.11-12. http://hdl.handle.net/2268/174166.

[27]. Mkrtchyan, G., Aleshin, V., Parkhomenko, Y., Kaehne, T., Luigi Di Salvo, M., Parroni, A., Contestabile, R., Vovk, A., Bettendorff, L., Bunik, V., 2015. Molecular mechanisms of the non-coenzyme action of thiamin in brain: biochemical, structural and pathway analysis. Sci. Rep. 5, 12583.

[28]. Nakagawasai, O., Yamadera, F., Iwasaki, K., Asao, T., Tan-No, K., Niijima, F., Arai, H., Tadano, T., 2007. Preventive effect of kami-untan-to on performance in the forced swimming test in thiamine-deficient mice: relationship to functions of catecholaminergic neurons. Behav. Brain Res. 177, 315-321.

[29]. Pan, X., Gong, N., Zhao, J., Yu, Z., Gu, F., Chen, J., Sun, X., Zhao, L., Yu, M., Xu, Z., Dong, W., Qin, Y., Fei, G., Zhong, C., Xu, T.L., 2010. Powerful beneficial effects of benfotiamine on cognitive impairment and $\beta$-amyloid deposition in amyloid precursor protein/presenilin-1 transgenic mice. Brain. 133, 1342-1351.

[30]. Pardo, M., Abrial, E., Jope, R.S., Beurel, E., 2016. GSK3 $\beta$ isoformselective regulation of depression, memory and hippocampal cell proliferation. Genes Brain Behav. 15, 348-355. 
[31]. Pepersack, T., Garbusinski, J., Robberecht, J., Beyer, I., Willems, D., Fuss, M., 1999. Clinical relevance of thiamine status amongst hospitalized elderly patients. Gerontology 45, 96-101.

[32]. Portari, G.V., Vannucchi, H., Jordao, A.A.Jr., 2013. Liver, plasma and erythrocyte levels of thiamine and its phosphate esters in rats with acute ethanol intoxication: a comparison of thiamine and benfotiamine administration. Eur. J. Pharm. Sci. 48, 799-802.

[33]. Rodríguez-Martín, J.L., Qizilbash, N., López-Arrieta, J.M., 2001. Thiamine for Alzheimer's disease. Cochrane Database Syst. Rev. 2, CD001498.

[34]. Sintoni, S., Kurtys, E., Scandaglia, M., Contestabile, A., Monti, B., 2013. Chronic valproic acid administration impairs contextual memory and dysregulates hippocampal GSK3 $\beta$ in rats. Pharmacol. Biochem. Behav. 106, 8-15.

[35]. Smidt, L.J., Creman, F.M., Grivetti, L.E., Clifford, A.J., 1991. Influence of thiamine supplementation on the health and general well-being of an elderly Irish population with marginal thiamine deficiency. J. Gerontol. 146, 16-22.

[36]. Strekalova, T., Sun, M., Sibbe, M., Evers, M., Dityatev, A., Gass, P., Schachner, M., 2002. Fibronectin domains of extracellular matrix molecule tenascin-C modulate hippocampal learning and synaptic plasticity. Mol. Cell. Neurosci. 21, 173-187.

[37]. Strekalova, T., Zörner, B., Zacher, C., Sadovska, G., Herdegen, T., Gass, P., 2003. Memory retrieval after contextual fear conditioning induces c-Fos and JunB expression in CA1 hippocampus. Genes Brain Behav. 2, 3-10. [38]. Strekalova, T., Gorenkova, N., Schunk, E., Dolgov, O., Bartsch, D., 2006. Selective effects of citalopram in a mouse model of stress-induced anhedonia with a control for chronic stress. Behav. Pharmacol. 17, 271-287.

[39]. Strekalova, T., Steinbusch, H.W., 2010. Measuring behaviour in mice with chronic stress depression paradigm. Prog. Neuropsychopharmacol. Biol. Psychiatry. 34, 348-361.

[40]. Strekalova, T., Evans, M., Chernopiatko, A., Couch, Y., Costa-Nunes, J., Cespuglio, R., Chesson, L., Vignisse, J., Steinbusch H.W., Anthony D.C., Pomytkin, I., Lesch, K.P., 2015a. Deuterium content of water increases 
depression susceptibility: the potential role of a serotonin-related mechanism. Behav. Brain. Res. 277, 237-244.

[41]. Strekalova, T., Evans, M., Costa-Nunes, J., Bachurin, S., Yeritsyan, N., Couch, Y., Steinbusch, H.M., Eleonore Köhler, S., Lesch, K.P., Anthony, D.C., 2015b. TIr4 upregulation in the brain accompanies depression- and anxietylike behaviours induced by a high-cholesterol diet. Brain. Behav. Immun. 48, 42-47.

[42]. Strekalova, T., Markova, N., Shevtsova, E., Zubareva, O., Steinbusch, H.M., Bachurin, S., Lesch, K.-P., 2016. Individual differences in behavioural despair predicts brain GSK3 $\beta$ expression in mice: the power of a modified swim test. J. Neural Plast. 2016;2016:5098591.

[43]. Sun, X.J., Zhao, L., Zhao, N., Pan, X.L., Fei, G.Q., Jin, L.R., Zhong, C.J., 2012. Benfotiamine prevents increased $\beta$-amyloid production in HEK cells induced by high glucose. Neurosci. Bull. 28, 561-566.

[44]. Vignisse, J., Steinbusch, H.W., Bolkunov, A., Nunes, J., Santos, A.I., Grandfils, C., Bachurin, S., Strekalova, T., 2011. Dimebon enhances hippocampus-dependent learning in both appetitive and inhibitory memory tasks in mice. Prog. Neuropsychopharmacol. Biol. Psychiatry 35, 510-522.

[45]. Vignisse, J., Steinbusch, H.W., Grigoriev, V., Bolkunov, A., Proshin, A., Bettendorff, L., Bachurin, S., Strekalova, T., 2014. Concomitant manipulation of murine NMDA- and AMPA-receptors to produce pro-cognitive drug effects in mice. Eur. Neuropsychopharmacol. 24, 309-320.

[46]. Vignisse J. Mécanisme des effets neuroprotecteurs de la thiamine et de précurseurs à plus grande biodisponibilité, PhD Theses, University of Liège, 2016. pp 1-243.19. http://hdl.handle.net/2268/174166.

[47]. Volvert, M.L., Seyen, S., Piette, M., Evrard, B., Gangolf, M., Plumier, J.C., Bettendorff, L., 2008. Benfotiamine, a synthetic S-acyl thiamine derivative, has different mechanisms of action and a different pharmacological profile than lipid-soluble thiamine disulfide derivatives. BMC Pharmacol. 8:10.

[48]. Zhang, G., Ding, H., Chen, H., Ye, X., Li, H., Lin, X., Ke, Z., 2013. Thiamine nutritional status and depressive symptoms are inversely associated among older Chinese adults. J. Nutr. 143, 53-58.

[49]. Zhao, Y., Wu, Y., Hu, H., Cai, J., Ning, M., Ni, X., Zhong, C., 2014. Downregulation of transketolase activity is related to inhibition of 
hippocampal progenitor cell proliferation induced by thiamine deficiency. Biomed. Res. Int. 2014, 572915.

[50]. Zhou, W., Chen, L., Yang, S., Li, F., Li, X., 2012. Behavioural stressinduced activation of FoxO3a in the cerebral cortex of mice. Biol. Psychiatry. 71, 583-592. 
Figure legends

Figure 1. Schematic of the timelines for each study. (A) modified swim test, (B) 5-day predation stress, (C) step-down avoidance, (D) fear conditioning test.

Figure 2. Thiamine and benfotiamine normalise floating and brain GSK3 $\beta$ expression in the modified swim test. Significant group differences were observed on day 5 of the modified swim test in (A) floating behaviour $\left({ }^{*} p<\right.$ 0.05 vs. Day2) and GSK3 $\beta$ mRNA levels as measured in (B) the hippocampus and $(C)$ prefrontal cortex $\left({ }^{*} p<0.05\right.$ vs. vehicle-treated, \#p $<0.05$ vs. naive mice).

Figure 3. Thiamine and benfotiamine prevent stress-induced changes in novelty exploration, anxiety-like behaviour, and brain GSK3 $\beta$ expression during predation stress. Significant group differences were found in the stressed animals in (A) novel cage rearing, (B) latency and (C) exit(s) to the open arms of the elevated O-maze. GSK3 $\beta$ mRNA levels in the (D) hippocampus and (E) pre-frontal cortex $\left({ }^{*} p<0.05\right.$ vs. vehicle-treated, \#p < 0.05 vs. naive mice).

Figure 4. Thiamine and benfotiamine enhance contextual conditioning and its extinction. The effect of thiamine and benfotiamine in the step-down avoidance study: (A) latency to step-down and (B) percentage of "good learners", (C) novel cage rearing. Freezing behaviour in the contextual fear conditioning paradigm after (E) "weak" and (D) "strong" training and (F) its extinction (\#p $<0.05$ vs. vehicle-treated, 1 h post-training; ${ }^{*} p<0.05$ vehicletreated, $24 \mathrm{~h}$ post-training). 


\section{Modified swim test}

2 weeks

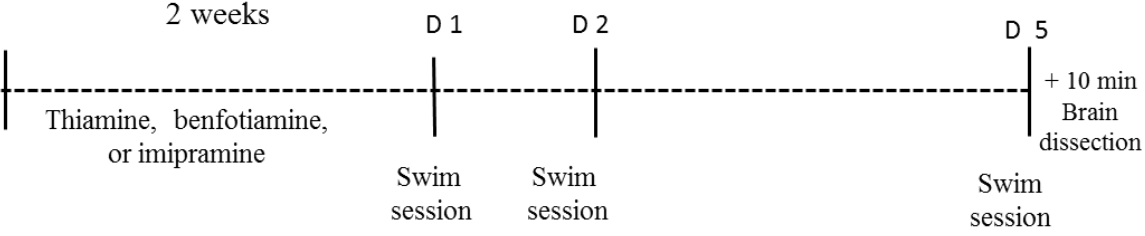

B

\section{Predation stress}

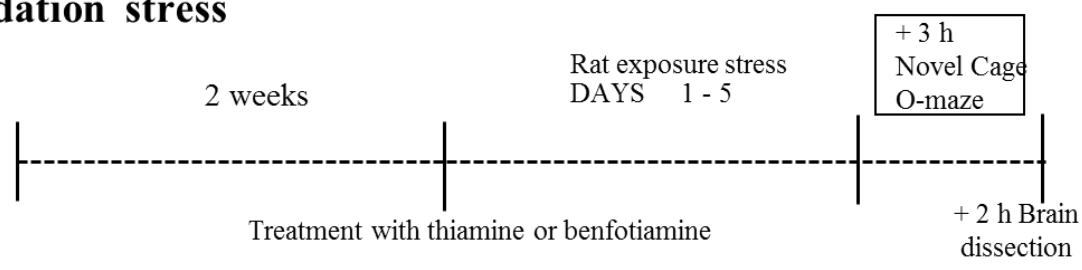

C

\section{Step-down avoidance test}

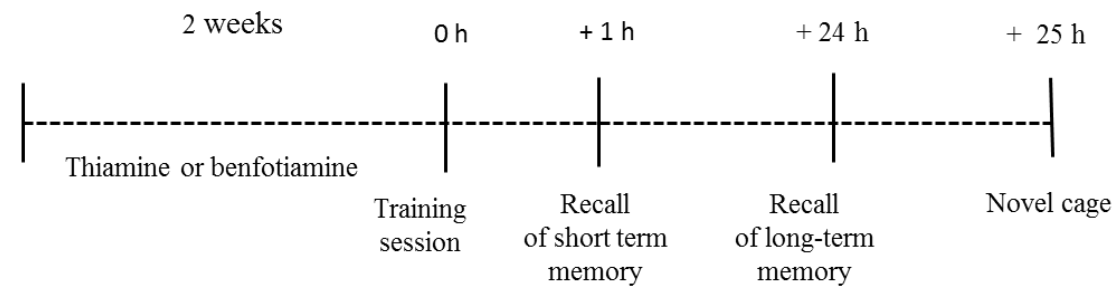

D

\section{Fear conditioning learning and extinction}

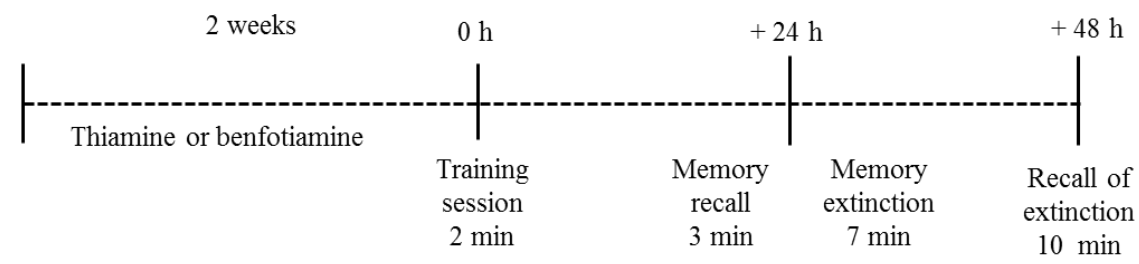

\section{Figure 1}




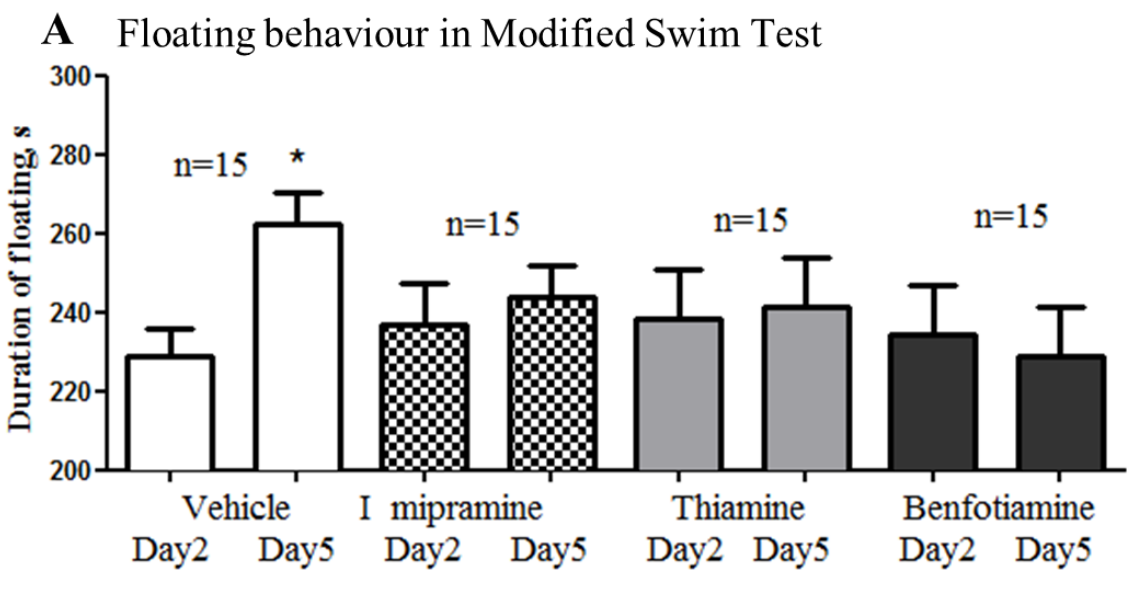

$\begin{array}{llll}\text { B } & \text { GSK-3 } \beta \text { mRNA: } & \text { C } & \text { GSK-3 } \beta \text { mRNA: } \\ \text { Hippocampus } & & \text { Prefrontal cortex }\end{array}$
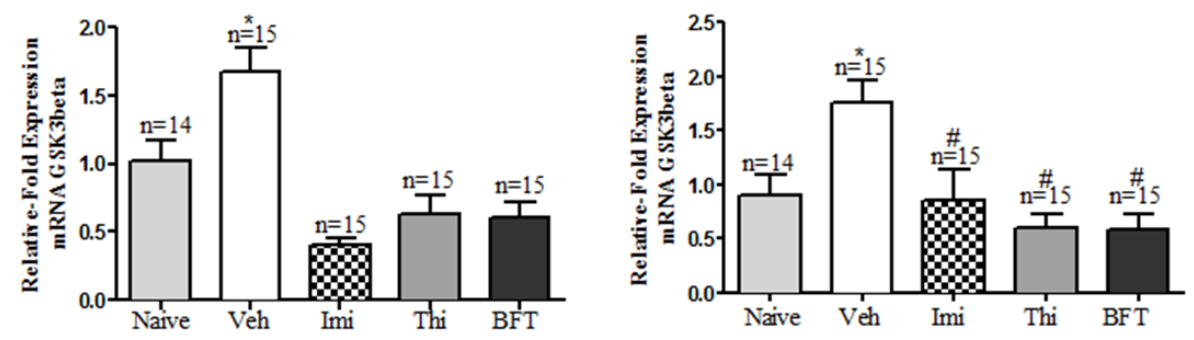

Figure 2 
A Rears in Novel Cage Test B Latency in O-Maze Test Exits in O-Maze Test
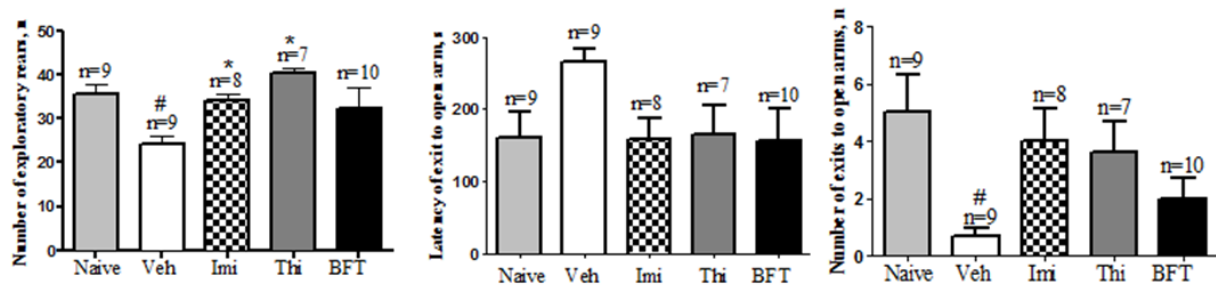

$\begin{array}{lll}\text { DSK-3 } \beta \text { mRNA: } & \text { E } & \text { GSK }-3 \beta \text { mRNA: } \\ \text { Hippocampus } & \text { Prefrontal cortex }\end{array}$
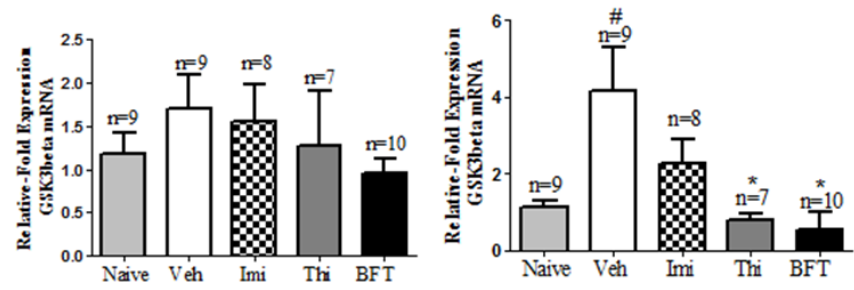

Figure 3 
A Latency in Step-Down Study

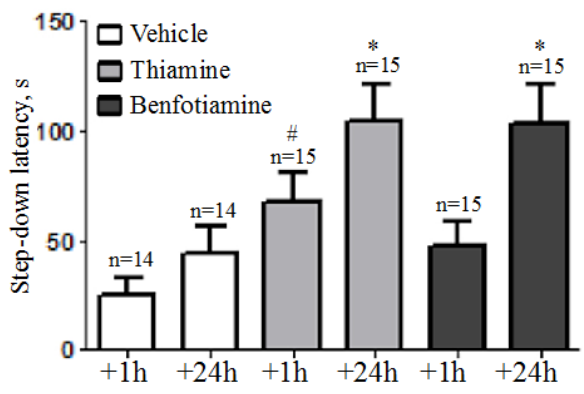

C Rears in Novel Cage Test

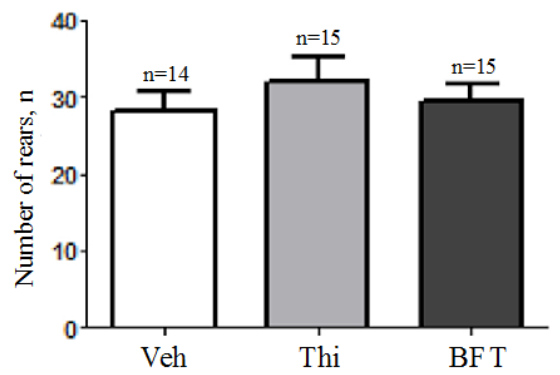

E Freezing during Recall Trial in 'Strong

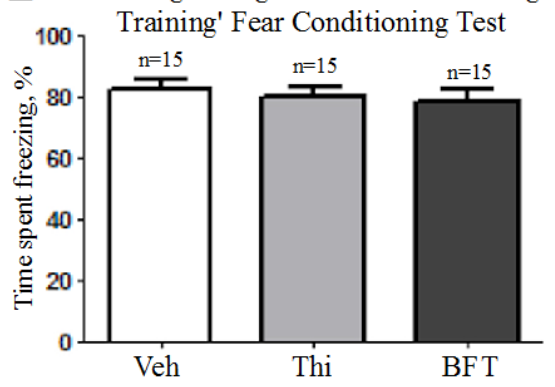

B Learning in Step-Down Study

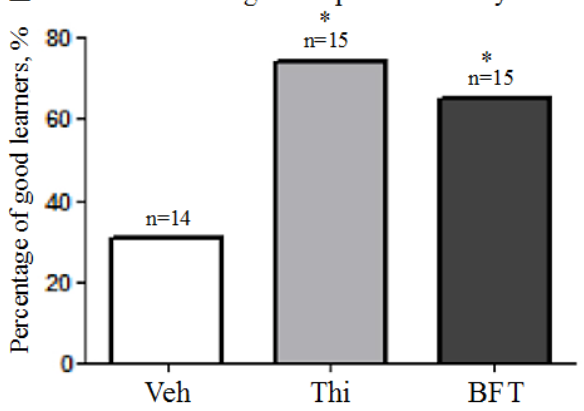

D Freezing during Recall Trial in 'Weak

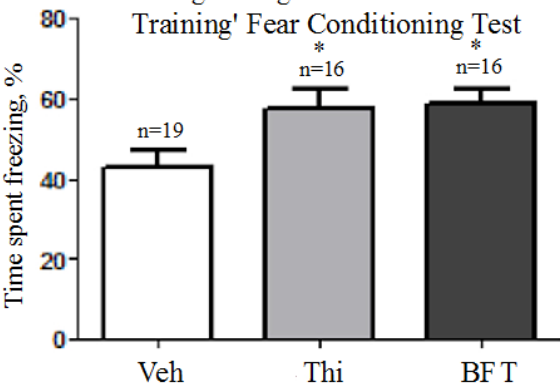

F Freezing during Extinction Trial in 100 'Strong Training' Fear Conditioning Test

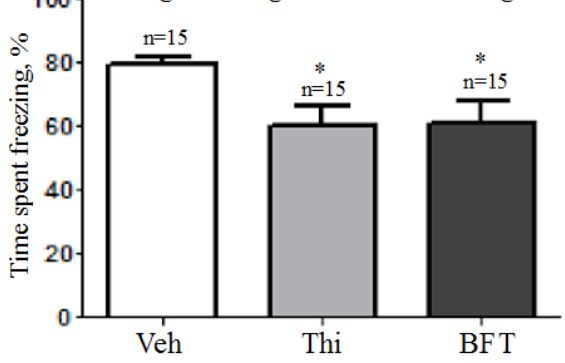

Figure 4 


\section{Supplementary Figure and Tables}

A TOTAL GSK3 $\beta$

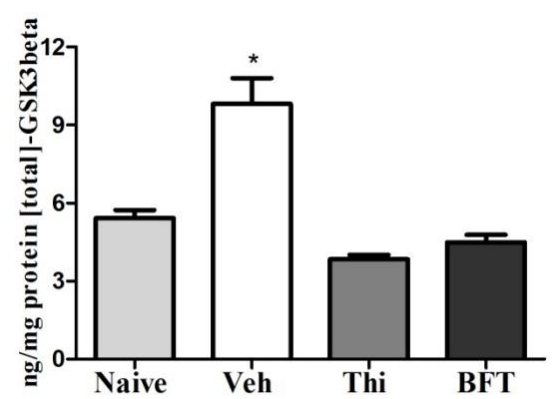

C p9SGSK3 $\beta /$ totalGSK3 $\beta$

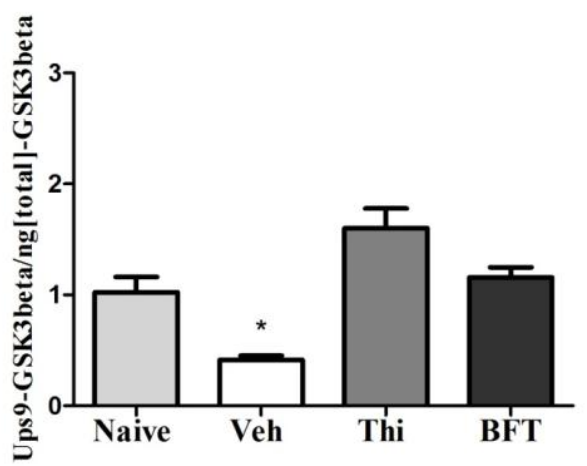

B

p9SGSK3 $\beta$

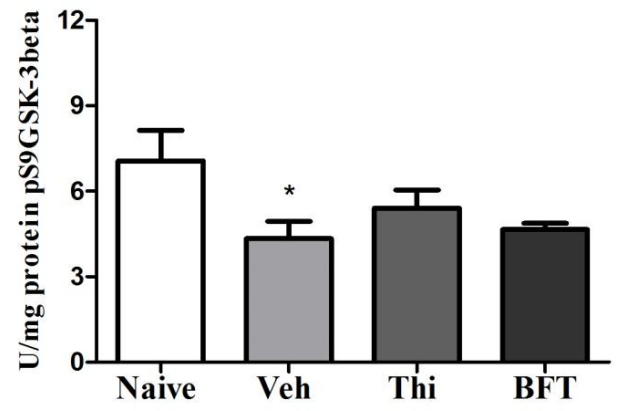

Figure 1. In the modified swim test, chronic treatment with thiamine and benfotiamine inhibits hippocampal increases in GSK3 $\beta$ activity. At day 5 graphs show the (A) total level of GSK3 $\beta,(B)$ the level of p9SGSK3 $\beta$ and (C) the ratio p9SGSK3 $\beta /$ totalGSK3 $\beta$. Group sizes as indicated on Fig. 2 B,C of the manuscript; ${ }^{*} p<0.05$ vs intact control, ANOVA and Tukey's multiple comparisons test (see Supplementary Table 4). Data are mean \pm SEM. 
A

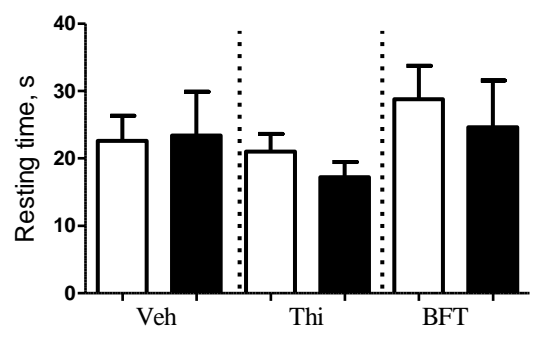

B

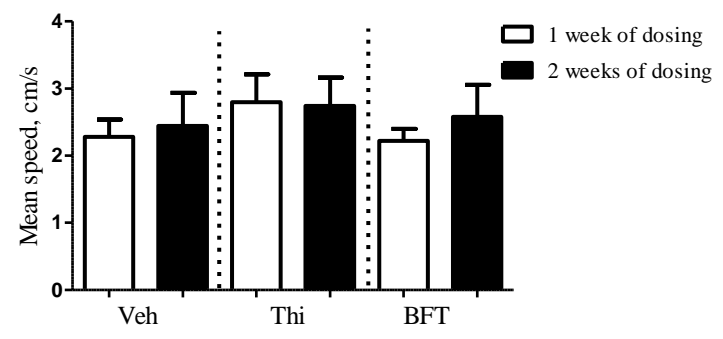

Figure 2. The effects of thiamine and benfortiamine on horizontal locomotion. Naïve mice (five mice per group) were treated with thiamine or benfortiamine via drinking water $(200 \mathrm{mg} / \mathrm{kg})$ or they no received drinking water supplement, and were tested after 1 week and after 2 weeks in True Scan activity boxes $(26 \mathrm{~cm} \times 26 \mathrm{~cm} \times 39 \mathrm{~cm}$; Coulbourn Instruments, Allentown, PA, USA) for $10 \mathrm{~min}$. Boxes were evenly illuminated with white light, with an illumination intensity of 25 Lux. Mean speed, defined as a distance covered during the experiment and a duration of resting, defined by an absence of crossing by an animal of more than $1,5 \mathrm{~cm}$ per a second, were scored automatically by red beam cells using TruScan software (Coulbourn), as described elsewhere (Strekalova et al., 2004, Malatynska et al., 2012, Couch et al., 2016). (A) mean speed of horizontal movements and (B) duration of resting time in the True Scan open field revealed that there was no difference between the groups at both time points ( $p>0.05$; ANOVA and Tukey post-hoc text). Data are mean \pm SEM. 
Table 1. Sequences of primers used

\begin{tabular}{|l|l|l|}
\hline Gene & Forward primer 5'-3' & Reverse primer 5'-3' \\
\hline GAPDH & TGCACCACCAACTGCTTAG & GGATGCAGGGATGATGTTC \\
\hline GSK3 $\beta$ & TCCATTCCTTTGGAATCTGC & CAATTCAGCCAACACACAGC \\
\hline
\end{tabular}

\section{Table 2A.}

\begin{tabular}{|l|l|l|l|}
\hline \multirow{2}{*}{ Groups } & \multicolumn{2}{|l|}{ Duration of floating } \\
\cline { 2 - 4 } & Day 1 & Day 2 & Day 5 \\
\hline $\begin{array}{l}\text { Vehicle-treated vs } \\
\text { Imipramine-treated }\end{array}$ & $\begin{array}{l}q=1.443 ; \\
p=0.7385, n s\end{array}$ & $\begin{array}{l}q=0.8933 ; \\
p=0.9213, n s\end{array}$ & $\begin{array}{l}q=2.217 ; \\
p=0.4063, n s\end{array}$ \\
\hline $\begin{array}{l}\text { Vehicle-treated vs } \\
\text { Thiamine-treated }\end{array}$ & $\begin{array}{l}q=1.834 ; \\
p=0.5697, n s\end{array}$ & $\begin{array}{l}q=0.9434 ; \\
p=0.9089, n s\end{array}$ & $\begin{array}{l}q=2.114 ; \\
p=0.4486, n s\end{array}$ \\
\hline $\begin{array}{l}\text { Vehicle-treated vs } \\
\text { BFT-treated }\end{array}$ & $\begin{array}{l}q=1.539 ; \\
p=0.6984, n s\end{array}$ & $\begin{array}{l}q=0.5075 ; \\
p=0.984, n s\end{array}$ & $\begin{array}{l}q=3.389 ; \\
p=0.0914, n s\end{array}$ \\
\hline $\begin{array}{l}\text { Imipramine-treated vs } \\
\text { Thiamine-treated }\end{array}$ & $\begin{array}{l}q=0.5791 ; \\
p=0.9766, n s\end{array}$ & $\begin{array}{l}q=0.1471 ; \\
p=0.9996, n s\end{array}$ & $\begin{array}{l}q=0.2123 ; \\
p=0.9988, n s\end{array}$ \\
\hline $\begin{array}{l}\text { Imipramine-treated vs } \\
\text { Benfotiamine-treated }\end{array}$ & $\begin{array}{l}q=2.686 ; \\
p=0.2421, n s\end{array}$ & $\begin{array}{l}q=0.2162 ; \\
p=0.9987, n s\end{array}$ & $\begin{array}{l}q=1.436 ; \\
p=0.7412, n s\end{array}$ \\
\hline $\begin{array}{l}\text { Thiamine-treated vs } \\
\text { Benfotiamine-treated }\end{array}$ & $\begin{array}{l}q=2.921 ; \\
p=0.1795, n s\end{array}$ & $\begin{array}{l}q=0.3262 ; \\
p=0.9956, n s\end{array}$ & $\begin{array}{l}q=1.095 ; \\
p=0.8658, n s\end{array}$ \\
\hline
\end{tabular}

Table 2A. Summary of comparisons between the groups that have received vehicle, or were administered with imipramine, thiamine or benfotiamine, on the duration of floating behaviour in the modified forced swim paradigm (one-way ANOVA and Tukey's multiple comparisons test). There was no significant group differences in duration of floating on Day 1 ( $F=0.9889$; $\mathrm{R}=0.0512, \mathrm{p}=0.40$ one-way ANOVA), Day $2(\mathrm{~F}=1.083 ; \mathrm{R}=0.0558, \mathrm{p}=0.3639$ one-way ANOVA) but on Day $5(F=4.211 ; R=0.1868, p=0.0094$ one-way ANOVA); ns, not significant. 
Table 2B.

\begin{tabular}{|l|l|}
\hline Groups & Day 2 vs Day 5 \\
\hline Vehicle-treated & $\mathrm{q}=5.877 ; \mathrm{p}=0.0018$ \\
\hline Imipramine-treated & $\mathrm{q}=2.058 ; \mathrm{p}=0.3412, \mathrm{~ns}$ \\
\hline Thiamine-treated & $\mathrm{q}=1.149 ; \mathrm{p}=0.7079, \mathrm{~ns}$ \\
\hline Benfotiamine-treated & $\mathrm{q}=0.1513 ; \mathrm{p}=0.9937, \mathrm{~ns}$ \\
\hline
\end{tabular}

Table 2B. Summary of repeated measures comparisons in changes of floating behaviour from Day2 to Day5 of the modified swim test between the groups that received vehicle, or were dosed with imipramine, thiamine or benfotiamine, on floating behaviour (Repeated Measures ANOVA and Tukey's multiple comparisons test). Note the vehicle-treated group showed significant differences in floating between time points ( $F=38.56$; $R=0.69, R M$ ANOVA), that were not found in imipramine-treated group ( $F=13.98 ; R=0.5$, RM ANOVA), nor in benfotiamine-treated mice ( $F=15.64 ; R=0.61, R M$ ANOVA), and in thiamine-treated group ( $F=8.61 ; R=0.38$, RM ANOVA); ns, not significant.

\section{Table 3.}

\begin{tabular}{|l|l|l|}
\hline \multirow{2}{*}{ Groups } & Structure \\
\cline { 2 - 3 } & Hippocampus & Prefrontal cortex \\
\hline $\begin{array}{l}\text { Naive mice vs } \\
\text { Vehicle-treated }\end{array}$ & $\mathrm{q}=4.939 ; \mathrm{p}=0.0078$ & $\mathrm{q}=4.439 ; \mathrm{p}=0.0259$ \\
\hline $\begin{array}{l}\text { Naive mice vs } \\
\text { Imipramine-treated }\end{array}$ & $\mathrm{q}=4.675 ; \mathrm{p}=0.0135$ & $\begin{array}{l}\mathrm{q}=0.2200 ; \\
\mathrm{p}=0.9999, \mathrm{~ns}\end{array}$ \\
\hline $\begin{array}{l}\text { Naive mice vs } \\
\text { Thiamine-treated }\end{array}$ & $\mathrm{q}=1.997 ; \mathrm{p}=0.245, \mathrm{~ns}$ & $\begin{array}{l}\mathrm{q}=1.353 ; \\
\mathrm{p}=0.8726, \mathrm{~ns}\end{array}$ \\
\hline $\begin{array}{l}\text { Naive mice vs } \\
\text { Benfotiamine-treated }\end{array}$ & $\mathrm{q}=2.653 ; \mathrm{p}=0.3414, \mathrm{~ns}$ & $\begin{array}{l}\mathrm{q}=1.458 ; \\
\mathrm{p}=0.8395, \mathrm{~ns}\end{array}$ \\
\hline
\end{tabular}




\begin{tabular}{|c|c|c|}
\hline $\begin{array}{l}\text { Vehicle-treated vs } \\
\text { Imipramine-treated }\end{array}$ & $q=9.613 ; p<0.0001$ & $q=4.524 ; p=0.0223$ \\
\hline $\begin{array}{l}\text { Vehicle-treated vs } \\
\text { Thiamine-treated }\end{array}$ & $q=7.872 ; p<0.0001$ & $q=5.536 ; p=0.0033$ \\
\hline $\begin{array}{l}\text { Vehicle-treated vs Benfotiamine- } \\
\text { treated }\end{array}$ & $q=6.865 ; p<0.0001$ & $q=5.646 ; p=0.0026$ \\
\hline $\begin{array}{l}\text { Imipramine-treated vs Thiamine- } \\
\text { treated }\end{array}$ & $q=1.742 ; p=0.7332, n s$ & $\begin{array}{l}q=1.110 \\
p=0.9333, n s\end{array}$ \\
\hline $\begin{array}{l}\text { Imipramine-treated vs Benfotiamine- } \\
\text { treated }\end{array}$ & $\mathrm{q}=1.882 ; p=0.8789, \mathrm{~ns}$ & $\begin{array}{l}q=1.213 \\
p=0.9103, n s\end{array}$ \\
\hline $\begin{array}{l}\text { Thiamine-treated vs Benfotiamine- } \\
\text { treated }\end{array}$ & $q=1.42 ; p>0.9999, n s$ & $\begin{array}{l}\mathrm{q}=0.09915 ; \quad \mathrm{p}> \\
0.9999, \mathrm{~ns}\end{array}$ \\
\hline
\end{tabular}

Table 3. Summary of group comparisons in brain GSK3 $\beta$ between naive mice and mice subjected to the modified swim test and treated with vehicle or treated with imipramine, thiamine or benfotiamine. There was a significant group difference in GSK3 $\beta$ level in the hippocampus ( $F=14.01 ; R=0.49, p<$ 0.0001 one-way ANOVA) and in the prefrontal cortex $(F=5.991 ; R=0.39, p<$ 0.001 one-way ANOVA); ns, not significant.

\section{Table 4.}

\begin{tabular}{|l|l|l|l|}
\hline \multicolumn{1}{|c|}{ Groups } & $\begin{array}{l}\text { Phosphorylated GSK3 } \beta \\
\text { (pS9GSK3 } \beta)\end{array}$ & Total GSK3 $\beta$ & $\begin{array}{l}\text { Ratio } \quad \text { pS9GSK3 } \beta \\
\text { /total GSK3 } \beta\end{array}$ \\
\hline $\begin{array}{l}\text { Naive mice vs } \\
\text { Vehicle-treated }\end{array}$ & $\begin{array}{l}\mathrm{q}=3.837 ; \\
\mathrm{p}=0.0683, \mathrm{~ns}\end{array}$ & $\begin{array}{l}\mathrm{q}=6.560 ; \\
\mathrm{p}=0.0005\end{array}$ & $\begin{array}{l}\mathrm{q}=5.061 ; \\
\mathrm{p}=0.009\end{array}$ \\
\hline $\begin{array}{l}\text { Naive mice vs } \\
\text { Imipramine-treated }\end{array}$ & $\begin{array}{l}\mathrm{q}=2.212 ; \\
\mathrm{p}=0.5280, \mathrm{~ns}\end{array}$ & $\begin{array}{l}\mathrm{q}=0.01275 ; \mathrm{p} \\
>0.9999, \mathrm{~ns}\end{array}$ & $\begin{array}{l}\mathrm{q}=0.4556 ; \\
\mathrm{p}=0.996, \mathrm{~ns}\end{array}$ \\
\hline $\begin{array}{l}\text { Naive mice vs } \\
\text { Thiamine-treated }\end{array}$ & $\begin{array}{l}\mathrm{q}=2.256 ; \\
\mathrm{p}=0.5085, \mathrm{~ns}\end{array}$ & $\begin{array}{l}\mathrm{q}=2.270 ; \\
\mathrm{p}=0.5044, \mathrm{~ns}\end{array}$ & $\begin{array}{l}\mathrm{q}=4.601 ; \\
\mathrm{p}=0.0207\end{array}$ \\
\hline $\begin{array}{l}\text { Naive mice vs } \\
\text { Benfotiamine-treated }\end{array}$ & $\begin{array}{l}\mathrm{q}=2.902 ; \\
\mathrm{p}=0.2595, \mathrm{~ns}\end{array}$ & $\begin{array}{l}\mathrm{q}=1.260 ; \\
\mathrm{p}=0.8982, \mathrm{~ns}\end{array}$ & $\begin{array}{l}\mathrm{q}=0.9586 ; \\
\mathrm{p}=0.9599, \mathrm{~ns}\end{array}$ \\
\hline
\end{tabular}




\begin{tabular}{|l|l|l|l|}
\hline $\begin{array}{l}\text { Vehicle-treated vs } \\
\text { Imipramine-treated }\end{array}$ & $\begin{array}{l}q=1.969 ; \\
p=0.6358, n s\end{array}$ & $\begin{array}{l}q=8.050 ; p< \\
0.0001\end{array}$ & $\begin{array}{l}q=5.640 ; \\
p=0.003\end{array}$ \\
\hline $\begin{array}{l}\text { Vehicle-treated vs } \\
\text { Thiamine-treated }\end{array}$ & $\begin{array}{l}q=1.666 ; \\
p=0.7637, n s\end{array}$ & $\begin{array}{l}q=10.30 ; p< \\
0.0001\end{array}$ & $\begin{array}{l}q=11.37 ; \\
p<0.0001\end{array}$ \\
\hline $\begin{array}{l}\text { Vehicle-treated vs } \\
\text { BFT-treated }\end{array}$ & $\begin{array}{l}q=0.4211 ; \\
p=0.9982, n s\end{array}$ & $\begin{array}{l}q=8.436 ; p< \\
0.0001\end{array}$ & $\begin{array}{l}q=6.168 ; \\
p=0.0011\end{array}$ \\
\hline $\begin{array}{l}\text { Imipramine-treated vs } \\
\text { Thiamine-treated }\end{array}$ & $\begin{array}{l}q=0.1780 ; \\
p>0.9999, n s\end{array}$ & $\begin{array}{l}q=2.714 ; \\
p=0.3272, n s\end{array}$ & $\begin{array}{l}q=6.056 ; \\
p=0.0013\end{array}$ \\
\hline $\begin{array}{l}\text { Imipramine-treated vs } \\
\text { Benfotiamine-treated }\end{array}$ & $\begin{array}{l}q=1.170 ; \\
p=0.9260, n s\end{array}$ & $\begin{array}{l}q=1.464 ; \\
p=0.8372, n s\end{array}$ & $\begin{array}{l}q=1.562 ; \\
p=0.8029, n s\end{array}$ \\
\hline $\begin{array}{l}\text { Thiamine-treated vs } \\
\text { Benfotiamine-treated }\end{array}$ & $\begin{array}{l}q=0.9737 ; \\
p=0.9579, n s\end{array}$ & $\begin{array}{l}q=0.9832 ; \\
p=0.9561, n s\end{array}$ & $\begin{array}{l}q=3.538 ; \\
p=0.1148, n s\end{array}$ \\
\hline
\end{tabular}

Table 4. Summary of group comparisons of brain phosphorylated and total forms of GSK3 $\beta$ and their ratio, between naïve mice, stressed vehicle-treated groups and stressed mice treated with imipramine, thiamine or benfotiamine. There was a significant group difference in the levels of total form of GSK3 $\beta$ ( $F=16.85 ; R=0.66, p<0.0001$ one-way ANOVA), but not a significant group difference in a level of phosphorylated form of GSK3 $\beta$ ( $F=2.023 ; R=0.16, p=0.11$ one-way ANOVA). There was a significant group difference in ratio of phosphorylated forms to total of GSK3 $\beta$ ( $F=9.545$; $\mathrm{R}=0.67, \mathrm{p}<0.0001$ one-way ANOVA); $n$, not significant.

\section{Table 5.}

\begin{tabular}{|l|l|}
\hline Groups & $\begin{array}{l}\text { Number of } \\
\text { exploratory rearings }\end{array}$ \\
\hline $\begin{array}{l}\text { Naive mice vs } \\
\text { Vehicle-treated }\end{array}$ & $\begin{array}{l}\mathrm{q}=5.381 ; \\
\mathrm{p}=0.0041\end{array}$ \\
\hline $\begin{array}{l}\text { Naive mice vs } \\
\text { Imipramine-treated }\end{array}$ & $\mathrm{q}=0.6816 ;$ \\
$\mathrm{p}=0.9886, \mathrm{~ns}$
\end{tabular}




\begin{tabular}{|c|c|}
\hline $\begin{array}{l}\text { Naive mice vs } \\
\text { Thiamine-treated }\end{array}$ & $\begin{array}{l}q=1.780 ; \\
p=0.7173, n s\end{array}$ \\
\hline $\begin{array}{l}\text { Naive mice vs } \\
\text { Benfotiamine-treated }\end{array}$ & $\begin{array}{l}q=1.344 \\
p=0.8751, n s\end{array}$ \\
\hline $\begin{array}{l}\text { Vehicle-treated vs } \\
\text { Imipramine-treated }\end{array}$ & $\begin{array}{l}q=4.449 \\
p=0.0245\end{array}$ \\
\hline $\begin{array}{l}\text { Vehicle-treated vs } \\
\text { Thiamine-treated }\end{array}$ & $\begin{array}{l}q=5.908 \\
p=0.0014\end{array}$ \\
\hline $\begin{array}{l}\text { Vehicle-treated vs } \\
\text { Benfotiamine-treated }\end{array}$ & $\begin{array}{l}q=3.050 \\
p=0.2173, n s\end{array}$ \\
\hline $\begin{array}{l}\text { Imipramine-treated vs } \\
\text { Thiamine-treated }\end{array}$ & $\begin{array}{l}q=2.263 ; \\
p=0.5060, n s\end{array}$ \\
\hline $\begin{array}{l}\text { Imipramine-treated vs } \\
\text { Benfotiamine-treated }\end{array}$ & $\begin{array}{l}q=0.7366 \\
p=0.9847, n s\end{array}$ \\
\hline $\begin{array}{l}\text { Thiamine-treated vs } \\
\text { Benfotiamine-treated }\end{array}$ & $\begin{array}{l}q=2.675 \\
p=0.3383, n s\end{array}$ \\
\hline
\end{tabular}

Table 5. Summary of comparisons of naive mice, stressed vehicle-treated groups and stressed mice treated with imipramine, thiamine or benfotiamine, on vertical exploratory activity in the novel cage test. There was a significant group difference in the number of exploratory rearings $(F=5.978 ; R=0.37, p<0.001$ one-way ANOVA); ns, not significant.

\section{Table 6.}

\begin{tabular}{|l|l|l|}
\hline Groups & $\begin{array}{l}\text { Latency of exit in the } \\
\text { open arm }\end{array}$ & Number of exits in the open arm \\
\hline $\begin{array}{l}\text { Naive mice vs } \\
\text { Vehicle-treated }\end{array}$ & $\begin{array}{l}\mathrm{q}=3.803 ; \\
\mathrm{p}=0.0740, \mathrm{~ns}\end{array}$ & $\begin{array}{l}\mathrm{q}=4.748 ; \\
\mathrm{p}=0.0144\end{array}$ \\
\hline $\begin{array}{l}\text { Naive mice vs } \\
\text { Imipramine-treated }\end{array}$ & $\begin{array}{l}\mathrm{q}=0.06827 ; \\
\mathrm{p}>0.9999, \mathrm{~ns}\end{array}$ & $\begin{array}{l}\mathrm{q}=1.045 ; \\
\mathrm{p}=0.9460, \mathrm{~ns}\end{array}$ \\
\hline $\begin{array}{l}\text { Naive mice vs } \\
\text { Thiamine-treated }\end{array}$ & $\begin{array}{l}\mathrm{q}=0.1527 ; \\
\mathrm{p}>0.9999, \mathrm{~ns}\end{array}$ & $\begin{array}{l}\mathrm{q}=1.176 ; \\
\mathrm{p}=0.9191, \mathrm{~ns}\end{array}$ \\
\hline
\end{tabular}




\begin{tabular}{|l|l|l|}
\hline $\begin{array}{l}\text { Naive mice vs } \\
\text { BFT-treated }\end{array}$ & $\begin{array}{l}q=0.09091 ; \\
p>0.9999, n s\end{array}$ & $\begin{array}{l}q=2.521 ; \\
p=0.3979, n s\end{array}$ \\
\hline $\begin{array}{l}\text { Vehicle-treated vs } \\
\text { Imipramine-treated }\end{array}$ & $\begin{array}{l}q=3.694 ; \\
p=0.0877, n s\end{array}$ & $\begin{array}{l}q=3.482 ; \\
p=0.1205, n s\end{array}$ \\
\hline $\begin{array}{l}\text { Vehicle-treated vs } \\
\text { Thiamine-treated }\end{array}$ & $\begin{array}{l}q=2.764 ; \\
p=0.3070, n s\end{array}$ & $\begin{array}{l}q=2.465 ; \\
p=0.4206, n s\end{array}$ \\
\hline $\begin{array}{l}\text { Vehicle-treated vs } \\
\text { Benfotiamine-treated }\end{array}$ & $\begin{array}{l}q=0.2051 ; \\
p=0.2297, n s\end{array}$ & $\begin{array}{l}q=1.120 ; \\
p=0.9313, n s\end{array}$ \\
\hline $\begin{array}{l}\text { Imipramine-treated vs } \\
\text { Thiamine-treated }\end{array}$ & $\begin{array}{l}q=0.2018 ; \\
p>0.9999, n s\end{array}$ & $\begin{array}{l}q=0.3267 ; \\
p=0.9993, n s\end{array}$ \\
\hline $\begin{array}{l}\text { Imipramine-treated vs } \\
\text { Benfotiamine-treated }\end{array}$ & $\begin{array}{l}q=0.03498 ; \\
p>0.9999, n s\end{array}$ & $\begin{array}{l}q=1.633 ; \\
p=0.7764, n s\end{array}$ \\
\hline $\begin{array}{l}\text { Thiamine-treated vs } \\
\text { Benfotiamine-treated }\end{array}$ & $\begin{array}{l}q=0.2051 ; \\
p=0.9999, n s\end{array}$ & $\begin{array}{l}q=1.132 ; \\
p=0.9290, n s\end{array}$ \\
\hline
\end{tabular}

Table 6. Summary of group comparisons between naive mice, stressed vehicle-treated animals and stressed mice treated with imipramine, thiamine-, or benfotiamine on parameters of anxiety in the O-maze test. There was a significant group difference in latent period of exit in the open $\operatorname{arm}(\mathrm{F}=2.649 ; \mathrm{R}=0.21, \mathrm{p}<0.05$ one-way ANOVA) and in number of exits in the open $\operatorname{arm}(\mathrm{F}=3.245 ; \mathrm{R}=0.25, \mathrm{p}<0.05$ one-way ANOVA); $\mathrm{ns}$, not significant.

\section{Table 7.}

\begin{tabular}{|c|c|c|}
\hline \multirow{2}{*}{ Groups } & \multicolumn{2}{|c|}{ Brain structure } \\
\hline & Hippocampus & Prefrontal cortex \\
\hline $\begin{array}{l}\text { Naive mice vs } \\
\text { Vehicle-treated }\end{array}$ & $\begin{array}{l}q=1.420 ; \\
p=0.8517, n s\end{array}$ & $\begin{array}{l}q=4.780 \\
p=0.0165\end{array}$ \\
\hline $\begin{array}{l}\text { Naive mice vs } \\
\text { Imipramine-treated }\end{array}$ & $\begin{array}{l}q=1.020 \\
p=0.9503, n s\end{array}$ & $\begin{array}{l}q=1.910 \\
p=0.6629, n s\end{array}$ \\
\hline $\begin{array}{l}\text { Naive mice vs } \\
\text { Thiamine-treated }\end{array}$ & $\begin{array}{l}q=0.2497 \\
p=0.9998, n s\end{array}$ & $\begin{array}{l}q=0.4737 \\
p=0.9971, n s\end{array}$ \\
\hline
\end{tabular}




\begin{tabular}{|l|l|l|}
\hline $\begin{array}{l}\text { Naive mice vs } \\
\text { Benfotiamine-treated }\end{array}$ & $\begin{array}{l}q=0.6404 ; \\
p=0.991, n s\end{array}$ & $\begin{array}{l}q=0.7616 ; \\
p=0.9825, n s\end{array}$ \\
\hline $\begin{array}{l}\text { Vehicle-treated vs Imipramine- } \\
\text { treated }\end{array}$ & $\begin{array}{l}q=0.3580 ; \\
p=0.9991, n s\end{array}$ & $\begin{array}{l}q=3.067 ; \\
p=0.2199, n s\end{array}$ \\
\hline $\begin{array}{l}\text { Vehicle-treated vs Thiamine- } \\
\text { treated }\end{array}$ & $\begin{array}{l}q=1.079 ; \\
p=0.9396, n s\end{array}$ & $\begin{array}{l}q=4.906 ; \\
p=0.0132\end{array}$ \\
\hline $\begin{array}{l}\text { Vehicle-treated vs } \\
\text { Benfotiamine-treated }\end{array}$ & $\begin{array}{l}q=2.098 ; \\
p=0.5793, n s\end{array}$ & $\begin{array}{l}q=4.691 ; \\
p=0.0192\end{array}$ \\
\hline $\begin{array}{l}\text { Imipramine-treated vs } \\
\text { Thiamine-treated }\end{array}$ & $\begin{array}{l}q=0.7145 ; \\
p=0.9864, n s\end{array}$ & $\begin{array}{l}q=2.246 ; \\
p=0.5164, n s\end{array}$ \\
\hline $\begin{array}{l}\text { Imipramine-treated vs } \\
\text { Benfotiamine-treated }\end{array}$ & $q=1.665 ;$ \\
$p=0.7639, n s$ & $q=2.320 ;$ \\
Thiamine-treated vs & $q=0.8524 ;$ \\
Benfotiamine-treated & $p=0.9738, n s$ & $q=0.3261 ;$ \\
\hline
\end{tabular}

Table 7. Summary of comparisons between naive mice, stressed vehicletreated groups and stressed mice treated with imipramine, thiamine, or benfotiamine in brain GSK3 $\beta$ mRNA. There was a significant group difference in the relative-fold expression of GSK3 $\beta$ in the prefrontal cortex $(F=4.658$; $R=0.39, p<0.01$ one-way ANOVA), but not in the hippocampus $(F=0.6893$; $R=0.07, \quad p=0.6$, one-way ANOVA); $n s$, not significant.

\section{Table 8.}

\begin{tabular}{|l|l|}
\hline Groups & Latency of step-down \\
\hline $\begin{array}{l}\text { Training: } \\
\text { Vehicle-treated vs Thiamine-treated }\end{array}$ & $\mathrm{q}=1.046 ; \mathrm{p}=0.7410, \mathrm{~ns}$ \\
\hline $\begin{array}{l}\text { Training: } \\
\text { Vehicle-treated vs Benfotiamine-treated }\end{array}$ & $\mathrm{q}=1.098 ; \mathrm{p}=0.7186, \mathrm{~ns}$ \\
\hline $\begin{array}{l}\text { Training: } \\
\text { Thiamine-treated vs Benfotiamine-treated }\end{array}$ & $\mathrm{q}=0.06234 ; \mathrm{p}=0.9989, \mathrm{~ns}$ \\
\hline $\begin{array}{l}\text { Recall+1h: } \\
\text { Vehicle-treated vs Thiamine-treated }\end{array}$ & $\mathrm{q}=4.045 ; \mathrm{p}=0.0152$ \\
\hline $\begin{array}{l}\text { Recall+1h: } \\
\text { Vehicle-treated vs Benfotiamine-treated }\end{array}$ & $\mathrm{q}=2.087 ; \mathrm{p}=0.3088, \mathrm{~ns}$ \\
\hline
\end{tabular}




\begin{tabular}{|l|l|}
\hline $\begin{array}{l}\text { Recall+1h: } \\
\text { Thiamine-treated vs Benfotiamine-treated }\end{array}$ & $\mathrm{q}=1.824 ; \mathrm{p}=0.4057, \mathrm{~ns}$ \\
\hline $\begin{array}{l}\text { Recall+24h: } \\
\text { Vehicle-treated vs Thiamine-treated }\end{array}$ & $\mathrm{q}=4.075 ; \mathrm{p}=0.0148$ \\
\hline $\begin{array}{l}\text { Recall+24h: } \\
\text { Vehicle-treated vs Benfotiamine-treated }\end{array}$ & $\mathrm{q}=4.023 ; \mathrm{p}=0.0163$ \\
\hline $\begin{array}{l}\text { Recall+24h: } \\
\text { Thiamine-treated vs Benfotiamine-treated }\end{array}$ & $\mathrm{q}=0.1037 ; \mathrm{p}=0.9970, \mathrm{~ns}$ \\
\hline
\end{tabular}

Table 8. Summary of group comparisons in the parameters of learning of the step-down avoidance task between vehicle-, thiamine- and benfotiaminetreated mice. There was a significant group difference in the latency of stepdown measured at the time point $+1 \mathrm{~h}$ post-training $(\mathrm{F}=4.117 ; \mathrm{R}=0.11, \mathrm{p}<$ 0.05 one-way ANOVA) and at time point $+24 \mathrm{~h}$ post-training ( $\mathrm{F}=5.740$; $\mathrm{R}=0.16$, $\mathrm{p}<0.01$ one-way ANOVA), but at the Training, i.e., at baseline conditions ( $F=0.3981 ; R=0.01, p=0.67$ one-way ANOVA); ns, not significant.

\section{Table 9.}

\begin{tabular}{|l|l|}
\hline Groups & Exact Fisher's test \\
\hline Vehicle-treated vs Thiamine-treated & $\mathrm{p}=0.0067$ \\
\hline Vehicle-treated vs Benfotiamine-treated & $\mathrm{p}=0.0360$ \\
\hline
\end{tabular}

Table 9. Summary of group comparisons in percentage of good learners in the step-down avoidance task between vehicle-, thiamine- and benfotiamine-treated mice. There was a significant difference in percentage of mice defined as "good learners" (latency of stepping down $>30 \mathrm{~s}$ at $+24 \mathrm{~h}$ post-training) in thiamine- and benfotiamine-treated groups, as compared with vehicle-treated controls.

\section{Table 10.}

\begin{tabular}{|l|l|}
\hline Groups & Number of exploratory rearings \\
\hline Vehicle-treated vs & $\mathrm{q}=1.469 ; \mathrm{p}=0.5569, \mathrm{~ns}$ \\
\hline
\end{tabular}




\begin{tabular}{|l|l|}
\hline Thiamine-treated & \\
\hline $\begin{array}{l}\text { Vehicle-treated vs Benfotiamine- } \\
\text { treated }\end{array}$ & $\mathrm{q}=0.5132 ; \mathrm{p}=0.9301, \mathrm{~ns}$ \\
\hline $\begin{array}{l}\text { Thiamine-treated vs Benfotiamine- } \\
\text { treated }\end{array}$ & $\mathrm{q}=0.9732 ; \mathrm{p}=0.7717, \mathrm{~ns}$ \\
\hline
\end{tabular}

Table 10. Summary of group comparisons between vehicle-, thiamine- and benfotiamine-treated mice in vertical activity measured in the novel cage test. There was no significant group difference in number of exploratory rearings ( $F=0.5606 ; R=0.03, p=0.58$ one-way ANOVA); $n s$, not significant.

Table 11.

\begin{tabular}{|l|l|l|l|}
\hline \multirow{2}{*}{ Groups } & \multicolumn{3}{|l|}{ Percentage of time spent with freezing during recall sessions } \\
\cline { 2 - 4 } & $\begin{array}{l}\text { "Weak" } \\
\text { training }\end{array}$ & "Strong" training & Memory extinction \\
\hline $\begin{array}{l}\text { Vehicle-treated vs } \\
\text { Thiamine-treated }\end{array}$ & $\begin{array}{l}q=3.448 ; \\
p=0.0474\end{array}$ & $\begin{array}{l}q=0.5708 ; \\
p=0.9143, n s\end{array}$ & $\begin{array}{l}q=3.606 ; \\
p=0.0378\end{array}$ \\
\hline $\begin{array}{l}\text { Vehicle-treated vs } \\
\text { Benfotiamine-treated }\end{array}$ & $\begin{array}{l}q=3.780 ; \\
p=0.0269\end{array}$ & $\begin{array}{l}q=1.047 ; \\
p=0.7410, n s\end{array}$ & $\begin{array}{l}q=3.465 ; \\
p=0.0477\end{array}$ \\
\hline $\begin{array}{l}\text { Thiamine-treated vs } \\
\text { Benfotiamine-treated }\end{array}$ & $\begin{array}{l}q=0.3112 ; \\
p=0.9737, n s\end{array}$ & $\begin{array}{l}q=0.4763 ; \\
p=0.9395, n s\end{array}$ & $\begin{array}{l}q=0.1412 ; \\
p=0.9945, n s\end{array}$ \\
\hline
\end{tabular}

Table 11. Summary of group comparisons between vehicle-, thiamine- and benfotiamine-treated mice in parameters of acquisition and extinction of contextual fear conditioning. There was a significant group difference in percent of time spent with freezing during a recall session after "weak" training ( $F=4.583 ; R=0.15, p<0.05$ one-way ANOVA) but not during "strong" training procedures $(F=0.2748 ; R=0.01, p=0.76$ one-way ANOVA). A significant difference between the groups in this parameter was observed during a recall of extinction procedure $(F=4.172 ; R=0.17, p<0.05$, one-way ANOVA); ns, not significant. 


\section{Chapter 6. Discussion and Societal Impact}

Key factors in the modified swim test: the role of timing, adversity, repeated testing and contextual reminders

Our data have supported the hypothesis that floating behaviour in additional swimming session of Porsolt's test in the mouse that is postponed from the initial noxious experience of swimming, might be a better predictor of individual depressive-like state than in classic tests, as it implements contextual learning. Despite a consideration that a repeated exposure to aversive context can reduce stress or memories about adversity, that may be related to the process of behavioural or neurophysiological habituation to a sensory stimulus resulting to lower floating in the repeated swim test (Natelson et al., 1988), we found an increase in his behaviour at the delayed repeated forced swim paradigm (Chapter 4). Moreover, this increase in a depressive-like behaviour was precluded by a two-week dosing with antidepressant treatment with low dose of imipramine in two studies (Chapter 4 and Chapter 5). Importantly, this treatment did not change floating behaviour on previous swim sessions suggesting distinct mechanisms of depressive-like state to be implicated in naïve versus experienced for forced swimming animals.

While repeated exposure to adversities including swimming under inescapable conditions has previously been used to achieve more robust depressive phenotypes by increasing the intensity of stress (Chrousos \& Gold, 1992; Krahe et al., 2002), the protocol of repeated swimming proposed here was designed on a different basis. In our study with "premature" exposure to swimming and analysis of GSK3 $\beta$ changes on Day 3 showed a lack of molecular changes that were typical for a conventional swim testing with a sacrifice on Day 5 (Chapter 4). Thus, our findings point to the critical role of timing in the development of molecular features of depressive-like behaviour. We defined the timing of the proposed protocol of modified swim test taking as a basis available data concerning a time curve of a consolidation of contextual fear memories in rodents (Johansen et al., 2011; Miller et al., 2014). Evidences suggest that with the mouse and rat 
paradigms modelling this phenomenon, the most common of which are the contextual fear conditioning model, the step-down avoidance test and fear potentiated startle, the neuronal processes of a fear consolidation last about 2 days post-conditioning of experimental animals (Anagnostaras et al., 2001; Strekalova et al., 2001, 2002; Katche et al., 2013). Similarly, our studies here revealed a critical time window of $48 \mathrm{~h}$ after two days of swimming exposure in the development of both behavioural and molecular manifestations of depressive-like state (Chapter 4).

A series of experiments that addressed the role of re-exposure of mice to a context of testing in our modified swim test, revealed its pivotal importance for the occurrence of behavioural and molecular changes observed in this paradigm. Study with a replacement of a swim session with an exposure to the context of testing alone on Day 2 and Day 5 have shown similar increases in GSK3 $\beta$ gene expression to those observed in a conventional protocol of this model (Chapter 4). These data, together with the finding that the omission of intermediate swimming or context exposure on Day 2 prevented an increase in floating (Chapter 4 ), demonstrate crucial role of contextual components in both behavioural and molecular depressive-like features, which are induced in our swim model. In previous experiments with reexposures of mice C57BI6 to a context, in which they have experienced adverse stimulations hours or days before, several key elements of the context which are substantial in their contextual conditioning, were identified (Strekalova et al., 2001, 2002). Experiments using similar to the presently used paradigm experimental designs with the step-down passive avoidance paradigm have shown that besides the immediate role of visual perception by mice of an experimental apparatus, such procedures as handling and a retrieval of a mouse from a cage, are important elements of contextual cues (Strekalova et al., 2001, 2002). As such, a re-exposure of an animal to the context, irrespectively to its duration, was per se a powerful reminding procedure, since it involves a retrieval of a mouse from a cage by the experimenter.

Together, our results supported a general assumption that similar factors to those that are crucial for acquisition of contextual memories in experimental 
models of learning, such as re-exposure to the context of testing, timing between challenges and development of behavioural response (Strekalova et al., 2001, 2002; Johansen et al., 2011; Miller et al., 2014), are also important for the depressive-like behavioural and molecular effects in the repeated swim test with delayed testing. Thus, proposed model has a number of similarities with models of contextual learning. As such, the mechanism of formation pathway of depression can be similar to the dynamics of molecular cascades of memory formation.

Changes of GSK3 $\beta$ brain activity as potential mechanism of enhanced learning, associated with depressive syndrome

As indicated above, the activity of GSK3 $\beta$ has been reported to play a key role in control of synaptic plasticity and memory function (Kimura et al., 2008b), consolidation of fear memory (Liu et al., 2010), the severity of PTSDlike symptoms (Dahlhoff et al., 2010), and is considered as a well-established marker of a depressive state (Silva et al., 2008; Oh et al., 2010). Based on this, we explored whether GSK3 $\beta$ expression and functions can correlate with behaviour of experimental mice in the modified forced swim test.

Indeed, the potentiation of floating in the modified swim test was accompanied by an up-regulation of brain GSK3 $\beta$ (Chapter 4), which was prevented by an antidepressant treatment with imipramine or thiamine and thiamine precursor benfotiamine (Chapter 4, Chapter 5). While analysis across experimental groups, naïve for pharmacological treatment revealed elevated expression of GSK3 $\beta$ in the hippocampus, but not in the prefrontal cortex. A differentiation of mice by floating behaviour into subgroups according to their scores of depressive-like behaviour showed that animals defined as "High floaters", displayed up-regulated GSK3 $\beta$ in the prefrontal cortex as compared to "Low floaters" (Chapter 4). There was a significant correlation between the duration of floating at Day 5 and GSK3 $\beta$ expression (Chapter 4). These results can be interpreted in a light of suggested key role of GSK3 $\beta$ in consolidation of fear memory (Dahlhoff et al., 2010; Wu et al., 2011). Moreover, some authors reported a potential involvement of changes in expression, of other kinases and transcription factors, in whose associated 
with GSK3 $\beta$ activity, such as the brain derived neurotrophic factor (BDNF; Kozlovsky et al., 2007; Krishnan et al., 2007) and adenylate cyclase activity and of recurrent activation of NMDA receptors (Cui et al., 2004), extracellular regulated kinases (ERK or mitogen-activated protein kinases, MAPK) and protein kinase B (Akt) (Ahi et al., 2004; Gould et al., 2008; Maguschak \& Ressler, 2008). The administration of imipramine, thiamine or benfotiamine that was shown to inhibit GSK3 $\beta$ under stress conditions (Chapter 5), and has abolished an over-expression of this molecule in the brain of mice exposed to delayed swimming session (Chapter 5). This showed that regardless a chemical class of compounds use, pharmacological suppression of expression of GSK3 $\beta$ results in normalized floating in the modified swim test.

A "premature" exposure to swimming and analysis of GSK3 $\beta$ changes on Day 3 showed a lack of behavioural and molecular effects that were typical for a conventional swim testing with a sacrifice on Day 5, thus, pointing to the critical role of GSK3 $\beta$ in "consolidation phase" for development depressivelike behavioural features (Chapter 4). On other hand, study with a replacement of a swim session with an exposure to the context of testing on Day 2 and Day 5 have shown similar increases in floating and GSK3 $\beta$ gene expression to those observed in a conventional protocol of this model (Chapter 4). These data, together with the finding that the omission of intermediate swimming or context exposure on Day 2 prevented an increase in both GSK3 $\beta$ expression and floating, further demonstrate GSK3 $\beta$ mediate pathologically enhanced consolidation of memories associated with adverse experience resulting in a depressive-like phenotype.

Increased of GSK3 $\beta$ mRNA levels in the hippocampus and prefrontal cortex of mice subjected to repeated swimming was accompanied by a substantial decrease of an inactive form of GSK3 $\beta$, its phosphorylated form, and the pS9-GSK3 $\beta / G S K 3 \beta$ ratio (Chapter 4), two well-established correlates of distress and depressive conditions (Beurel et al., 2015). The profound decrease of these parameters in both the hippocampus and prefrontal cortex further emphasizes the role of repeated swimming in the development of depressive-like behavioural and molecular changes 
observed in current study. Importantly, antidepressant treatments with imipramine, thiamine and benfotiamine used in another study revealed their normalizing effects on brain GSK3 $\beta$ levels and pS9-GSK3 $\beta / G S K 3 \beta$ ratio (Chapter 5) thus, further supporting our conclusion.

We found that pS9-GSK3 $/$ GSK3 $\beta$ ratio was similar on Day 2 and Day 5 of testing and no differences in these measures was found between "High" and "Low" floaters, that possibly reflects limited sensitivity of our assay (Chapter 4). The discrepancy between gene and protein parameters of GSK3 $\beta$ function on Day 2 might also be due to distinct dynamics of changes of gene expression and phosphorylation of GSK3 3 . We recently showed that, while mice sacrificed $24 \mathrm{~h}$ post-swim in the modified swimming test display no changes in GSK3 $\beta$ at the gene expression level, they have elevated GSK3 $\beta$ protein levels, suggesting that gene and protein changes in GSK3 $\beta$ may not always occur in parallel. Generally, these results further emphasize the role of repeated versus single exposures to adverse contexts in the regulation of activity of brain GSK3 $\beta$.

Different roles of the hippocampus and the prefrontal cortex in behavioural and molecular changes in the modified swim test

In the modified forced swim test, significant correlation between floating behaviour and GSK3 $\beta$ mRNA levels was found in the prefrontal cortex, but not in the hippocampus (Chapter 4). Increased level of GSK3 $\beta$ mRNA found in the prefrontal cortex of "High floaters", but not in a subgroup of mice classified as "Low floaters" (Chapter 4). Besides, exposure of mice to a context of swimming alone, without swim session, resulted in increased gene expression of GSK3 $\beta$ in the prefrontal cortex, but not in the hippocampus (Chapter 4). Together, these data suggest a key role of this brain structure in the regulation of enhanced contextual learning of adversities and are in line with previously published literature demonstrating critical functions of the prefrontal cortex in effective coping with stress (Amat et al., 2005; Sotres-Bayon \& Quirk, 2010; Warden et al., 2012). These data are also in line with clinical findings, showing a predominant overactivation of prefrontal cortex in patients with depression (Mayberg et al., 
1999; Nemeroff et al., 2006; Etkin, 2010; Holtzheimer \& Mayberg, 2011). Animal studies demonstrated that excitatory synapses in neurons of the prefrontal cortex are weakened in mice that are resilient to helplessness in chronic unpredictable stress, and optogenetic activation of its neurons in rodents induces depression-like effects (Warden et al., 2012; Yizhar et al., 2011; Zhang et al., 2016).

At the same time, the prefrontal cortex is known to regulate the processes underlying contextual fear (Giustino \& Maren, 2015; Rozeske et al., 2015). For example, a retrieval of a remote contextual fear memory has elevated the expression of plasticity-associated c-Fos and zif268 genes exclusively in the prefrontal cortex (Frankland et al., 2004). Classical experiments with contextual fear memory paradigms showed that a retrieval of a memory a few days after conditioning is dependent on the function of the prefrontal cortex, while the hippocampus is predominantly involved in the acquisition and consolidation of these memories (Frankland \& Bontempi, 2005; Sutherland et al., 2010; Dudai et al., 2012; Rozeske et al., 2015).

Our experiments have revealed an over-expression of GSK3 $\beta$ in the hippocampus of experimental mice, which did not differ between "High" and "Low floaters" (Chapter 4), suggesting that this brain structure may be predominantly involved in a recall of a context during testing in the modified swim model. This speculation is based on the views of a well-established role of the hippocampus in the mechanisms of contextual memory (Kim \& Fanselow, 1992; Anagnostaras et al., 2001; Phillips \& LeDoux, 2009). Furthermore, as it was discussed above, GSK3 $\beta$ is a well-established regulator of hippocampal plasticity, a deficit of which resulted on impaired long-term potentiation and memory. As such, in the modified forced swim model, where the contextual cues have been shown critical in the potentiation of floating behaviour and associated molecular changes, similar level expression of hippocampal GSK3 $\beta$ of "High floaters" and "Low floaters" mice may be interpreted as a molecular signature of a similar context recall in two groups that is not related to the development of an individual "depressive-like state". 
However, several facts speak against this speculation. In the modified forced swim test, the administration of antidepressant imipramine precluded changes in the GSK3 $\beta$ specifically in the hippocampus but not in the prefrontal cortex (Chapter 4). This effect was found both at the gene and protein levels. In addition, antidepressant treatments with thiamine and benfotiamine have resulted to a suppression of hippocampal activities of GSK3 3 (Chapter 5). Thus, the role of the hippocampus in the development of a depressive-like state associated with enhanced contextual acquisition of negative memories is likely to be more complex than merely an involvement in contextual learning. Available literature supports this view (Omata et al., 2011; Li et al., 2014). As for instance, an infusion of GSK3 $\beta$ activator into the dentate gyrus of the hippocampus abolished the antidepressant-like effects of butyrolactone and roscovitine in the model of learned helplessness.

Together, our results suggest that both the hippocampus and the prefrontal cortex are implicated in the formation of a depressive-like state associated with enhanced memory of aversive events in the proposed modified swim model, but they role and dynamics of the involvement in these processes are different. Further studies are required to address these issues experimentally.

\section{Modeling of individual variability in the susceptibility to a depressive-like state with the modified swim test: a comparison with other paradigms}

In the modified forced swim test, mice display a substantial inter-individual variability in floating and molecular changes, the markers of a depressivelike state, that is in line with the outcome from other animal studies on stress and depression (Strekalova et al., 2004, 2011; Wüst et al., 2005; Gorka et al., 2016; Monestier et al., 2016). In comparison to the classical Porsolt's test, the modified protocol with additional delayed session did enable a differentiation of individual animals upon their susceptibility to a depressivelike state. Behavioural alterations in floating were accompanied by molecular changes that so far were achieved with a limited number of models. We found that inbred mice can be separated into "High" and "Low floaters" that paralleled changes in the GSK3 $\beta$ expression (Chapter 4). No 
correlation between floating and expression data were found after one or two sessions of forced swimming (Chapter 4 and Chapter 5). This is similar, as for instance, to a separation to anhedonic and nonhedonic subgroups in stress-induced anhedonia models (Strekalova et al., 2004; Bergström et al., 2008; Wiborg et al., 2013), resilient and susceptible to stress-induction phenotype of social avoidance (Krishnan et al., 2007), rats with Low- and High active avoidance profiles in the learned helplessness paradigms (Carlson \& Glick, 1991).

Studies of the last decade have identified the epigenetic and posttranslational mechanisms as important actors in depressive-like state and susceptibility to stress, that potentially may explain inter-individual differences in physiological responses of genetically inbred mice exposed to modified swim test in our studies. They include histone methylation (Lolak et al., 2014) and histone acetylation (Covington et al., 2009), palmitoylation of various receptors and neuronal factors (Ponimaskin et al., 2008), nucleotide modifications (Suzuki \& Bird, 2008) and hydroxymethylation (Kriaucionis \& Heintz, 2009), chromatin regulation (Wilkinson et al., 2009), mechanisms mediated by micro RNA (Higuchi et al., 2016), transcriptional dysregulation (Peña et al., 2014). As for instance, chronic social defeat stress increased repressive histone 3 lysine 14 (H3K14) acetylation, that can be reversed by chronic antidepressant treatment (Wilkinson et al., 2011). Antidepressant treatment was shown to increase $\mathrm{H} 3$ acetylation at BDNF promoter that correlated with elevated hippocampal expression of BDNF, an important factor of a depressive-like state (Bliss \& Collingridge, 1993; Tsankova et al., 2006).

On gene level, several genes were by now identified to be associated with elevated susceptibility to stress and thus potentially can be involved in the enhanced learning of adverse experiences as well. These genes are the serotonin transporter (SERT) (Lesch \& Mössner, 2006; Reinelt et al., 2015), nuclear receptor related 1 protein (Nurr1) (Montes et al., 2016), neurotrophic tyrosine kinase, receptor, type 3 (NTRK3) (Farhang et al., 2014). Data from a genome-wide association study revealed genetic effects of intronic SNP of the glucocorticoid receptor (GR) on emotional memory in 
volunteers. Increased cortisol sensitivity of the GR and enhanced glucocorticoid signaling under stress were related to enhanced memory of adversities (Hauer et al., 2011). Homozygous carriers of the Bcll G allele, associated with low plasma cortisol levels, had a significantly increased risk for traumatic memories and PTSD symptoms of chronic anxiety, pain, depression and impaired health-related quality of life after cardiac surgery, accompanied by the increased cortisol sensitivity (Hauer et al., 2011). While our study has identified altered expression of GSK3 $\beta$ in the cohorts of mice with inter-individual differences in memorizing of adverse experiences, no direct evidences for the involvement of this mechanism in the individual susceptibility, at the level of genetic or epigenetic regulation, was reported in the available literature. However, genetic studies have suggested the association of GSK3 $\beta$ polymorphisms with various phenotypes of mood disorders (Grimes \& Jope, 2001; Jope \& Roh, 2006; Saus et al., 2010).

As it was mentioned above, individual profiles of depressive-like behaviour in the modified swim test can be considered as a manifestation of active and passive coping with stress (De Boer et al., 1990; Strekalova, 1995). Passive strategy of coping is characterized by an immobility and suppression of environmentally-directed activities, while active style is characterized by active responding whereby the animal displays much locomotor activity in attempting to escape from or to deal with an external threat. The interindividual differences in coping with stress were found to be associated with altered expression arginine-vasopressin receptor 1 , oxytocin and others. It can be anticipated, that these molecular factors are differently expressed in "Low" and "High floaters" in the modified swim test.

Whether or not cortisol levels change in the modified swim test was not study yet, but such studies are on the way. Previously, the magnitude of the individual corticosterone response to the initial stress exposure in rodents was found to positively relate to the responses to exposures (Natelson et al., 1988; Baganz et al., 2010) that suggest similar changes in "High floaters" in our model. We hypothesize that factors that regulate the hypothalamicpituitary-adrenal system, such as, for example, newly discovered FK506binding Protein 51 (FKBP51) encoding a chaperon protein, regulating GR- 
related processes can be a mediator of inter-individual variability in our model (Raabe \& Spengler, 2013).

\section{A comparison of the modified swim test against other depression paradigms: pharmacological sensitivity, etiological relevance, face and construct validity}

The key criteria of animal model of human disorders were proposed while ago and are considered to comprise criteria of etiological relevance, face and construct validity and pharmacological sensitivity (McKinney \& Bunney, 1969; McArthur \& Borsini, 2006). Etiological validity is a relevance of model is the extent to which it mimics the causation and / or origins of the disease. Face validity is a degree of the similarity of phenomenological pathologies observed in an animal and in man. Construct validity is an ability of the model to reproduce the similar pathophysiological changes in animals to those that are observed in a man after similar challenges. Pharmacological sensitivity of animal model is a similarity of response to pharmacotherapies between experimentally-induced pathology in a lab and in a clinic.

Short tests for depressive-like behaviour, such as the Porsolt's swim and tail suspension tests, are broadly used in translational research on depression (Cryan et al., 2005; Overstreet, 2012; Willner \& Belzung, 2015). However, they are criticized for insufficient validity and etiological relevance. Moreover, discrepant effects of compounds with antidepressant activity between these tests and other paradigms of depression, not uncommon false-positive and false-negative outcomes with drug testing suggest limited validity of classic variants of the forced swim model (Perrault et al., 1992; Cryan et al., 2005; Harro et al., 2011; Ampuero et al., 2015). In line with these data, our study with the administration of $\mathrm{T} 2$ have revealed an antidepressant effect of this hormone at two doses in two different mouse lines in the two-day variants of the tail suspension but not in the forced swim test (Chapter 2, Chapter 3). In particular, we found that at the dose $0.75 \mathrm{mg} / \mathrm{kg}$, an intraperitoneal administration of $\mathrm{T} 2$ has decreased depressive-like behaviour in the tail suspension test measured by the immobility time, but did not change the values of the floating behaviour in the forced swim test 
(Chapter 2, Chapter 3). A lack of significant behavioural effects of $\mathrm{T} 2$ in the forced swim test was evidenced both in C57BI/6 and CD1 mouse strains. The administration of other dose of T2, such as $0.25 \mathrm{mg} / \mathrm{kg}$ and $1.5 \mathrm{mg} / \mathrm{kg}$ was found ineffective in inducing any behavioural changes in this paradigm (Chapter 3). Yet, the involvement of T2-related mechanisms in the CNS was demonstrated in the chronic stress paradigm of stress-induced anhedonia (Chapter 2) and was further confirmed in the tail suspension test (Chapter 2) in which standard antidepressant treatments have produced similar effects, thus, additionally supporting our findings.

On a different note, our studies with cross-validation of the tail suspension and the forced swim test have resulted to the identification of T2 as an antidepressant agent. These data with classic tail suspension test for the first time demonstrated that $\mathrm{T} 2$, which previously was considered as an inactive molecule, could be exploited clinically. These studies also suggest that the development of more refined methodological variants of the forced swim test is needed for more accurate pre-clinical applications of this model.

Proposed here modified forced swim test can be considered as a paradigm of depression with substantial etiological relevance, since it mimics such important pathophysiological factor as enhanced cognitive processing of adversities (Gold \& Korol, 2012; Flores et al., 2014). Besides, repeated exposures to negative inescapable experiences is regarded as a substantial factor of a development of a depressive-like state (Billings et al., 1983; Taché et al., 2015; Ahnaou and Drinkenburg, 2016; Kim et al., 2016;). Also, the uncontrollability of external stressors, which is characteristic for currently proposed modified swim test, is well documented to be the element of the pathophysiology of experimentally induced depressive condition (Willner et al., 1984; Job \& Barnes, 1995; Havranek et al., 2016). While proposed by us paradigm does not allow differentiate whether increased acquisition, consolidation or recall of these negative memories play a role, it models increased neurobiological depressive-like molecular response in the limbic system of individual animals which tend to display exaggerated memories for negative stimulation. 
Besides, it can be concluded that modified forced swim test has high face validity in modeling depressive state. Repeated sessions in the modified forced swim test were designed to produce a state of learned helplessness, one of the main behavioural hallmarks of depression (De Pablo et al., 1989), as well as a state of despair, that is characteristic symptom for patients with this condition. In a contrary, the effects of single-task sessions with the Porsolt's and tail suspension tests are limited in the induction of these behaviours and most importantly, do not mimic a "consolidation" phase of negative experiences. As a result, in contrast to classic variants of forced swim model, experimental animals exposed to the modified swim test, have displayed depressive behaviour that significantly correlated with an independent marker of this state, GSK3 $\beta$, in the structures of the limbic system of the brain (Jope \& Roh, 2006; Diniz et al., 2011; Ronai et al., 2014). A significant correlation between depressive-like behaviour comprising in helpless behaviour and over-expression of one of the most established molecular hallmarks of depression suggest high face validity of newly proposed model.

With respect to construct validity, we showed that repeated, but not single exposures result in increased activities of GSK3 $\beta$ on a protein level (Chapter 4). Human post-mortem studies revealed elevated activities of GSK3 $\beta$ in the hippocampus (Oh et al., 2010) and prefrontal cortex (Karege et al., 2007), as well as lower levels of phosphorylated (inactive) form of GSK3 $\beta$ in depressed patients (Polter et al., 2010).

Finally, our data suggest high validity of the modified swim test in terms of pharmacological sensitivity. In our studies, both behavioural and molecular changes were prevented by two-week treatment with low dose of antidepressant treatment, such as $7.5 \mathrm{mg} / \mathrm{kg} /$ day of orally administrated imipramine. At this dose, floating behaviour in the classic variants of the swim test was not altered in our experiments (Chapter 4, Chapter 5) nor in similar previously reported studies (Borsini \& Meli, 1988; Tohda et al., 2010; Elgarf et al., 2014). In the classic Porsolt's test, similarly low doses of imipramine $5-10 \mathrm{mg} / \mathrm{kg}$ were effective only in combination with other antidepressant treatments, such as amantadine (Rogóz et al., 2007), 
metyrapone (Rogóz et al., 2003) or during exposure to an enriched environment (Possamai et al., 2014). Similar to our work, it was found that the administration of imipramine to rats enhanced an antidepressant-like effect of enriched environment during the delayed swim session on eighth day, but not on first or second days (Possamai et al., 2014). A body of evidence showed that tricyclic alters depressive-like behaviours at the dose $10-30 \mathrm{mg} / \mathrm{kg}$ but not at the range of concentrations used in our work. Thus, proposed here model exerted increased pharmacological sensitivity. This can be very important issue, since it potentially enables to improve drug research and discovery and refines pharmacological testing of new compounds.

Together, we believe that the proposed forced swim test protocol satisfied the criteria of validity of animal model of depression.

\section{Advantages of modified swim test over other models of depression}

As it was mentioned above, similarly to the modified forced swim test, Porsolt's and tail suspension tests imply an exposition to inescapable, uncontrollable, unpredictable aversive and potentially dangerous event, which with can also apply repeatedly. However, these tests do not mimic a consolidation phase of adverse memories and comprise fewer exposures to adversities. Both factors seem to be critical, since molecular marker of a depressive-like state GSK3 $\beta$ was found to be elevated on gene expression level only after additional delayed exposure, as our study showed (Chapter 4). Furthermore, significant correlation between brain expression of GSK3 $\beta$ and floating behaviour, a sign of a depressive-like state was only found during a delayed but not classic time points of testing (Chapter 4). On a protein level, GSK3 $\beta$ activities were elevated, that suggests a role of repeated exposures to forced swimming to be a factor of a development of a depressive-like state, however, no correlation was found between this measure and floating behaviour (Chapter 4). Thus, these two classic paradigms seemingly possess lower etiological relevance, as compared to newly proposed modified swim test. As such, modified forced swim test can be compared it is etiological validity and relevance to the models with 
chronic repeated exposures to stress such as chronic mild stress (Strekalova et al., 2004; Chen et al., 2010), learned helplessness (Seligman \& Beagley, 1975; De Pablo et al., 1989), whose etiological relevance is well-established.

Repeated uncontrollable stress, an element of the modified swim model, is well described to induce wide variety of substantial features of depression, such as the escape learning impairment, deficits in exploration, and distinct neuroendocrinological changes (Zacharko \& Anisman, 1991; Holsboer, 2000; Chourbaji et al., 2005; Vollmayr et al., 2007). Similarly, our paradigm and other models with repeated swimming are likely to evoke these elements of depressive-like state, that need to be investigated in future experiments.

Finally, proposed model, for the first time, may enable a tracking of an exact temporal relationship between an event that induces a depressive-like state and a development its molecular / neurobiological trace in dynamics that in such sense, is virtually impossible with chronic models of depression.

As we conclude, our study showed that in comparison to the classical one- or two-day forced swim testing, a proposed modification of the swim test with additional delayed session can enable to: 1) mimic changes of pivotal molecular marker of depression such as brain GSK3 $\beta$ activity; 2 ) differentiate of individual animals upon their susceptibility to a depressive-like state by behavioural measures or correlating with them molecular changes that so far was achieved only with chronic experimental paradigms; 3) model a phenomenon of enhanced contextual learning in the pathogenesis of individual vulnerability to a development of depression; 4) increase a sensitivity of measured behavioural and molecular parameters of a depressive-like state to the effects of subtle antidepressant manipulations, which are not detectable in classical versions of the forced swim test.

Taken together, the paradigm of modified swimming test offers a tool to study the role of enhanced cognitive processing in the pathophysiological mechanisms of depression and inter-individual differences in the susceptibility to this condition in mice. The modified swim test can be comparable to chronic depression paradigms, e.g., chronic stress, learned helplessness and similar models, which possess substantial construct validity 
and etiological relevance that cannot be attributed to short behavioural tests for a depressive-like behaviour, like classic Porsolt's and tail suspension tests. In sum, it combines the advantages of classical models of depression that possess high validity but known to be labour and time demanding.

Highlighting supplementary therapies of depression: the role of GSK3 $\beta$ in mechanisms of depression, enhanced learning of advertises and antidepressant therapies

Because of thiamine is involved both in the neurobiology of cognitive deficits and depression and effect on GSK3 $\beta$ activity (Pan et al., 2010), we also studied effects of thiamine and the more bioavailable its precursor benfotiamine on depressive-like behaviour in the modified forced swim test. Deficiencies in thiamine metabolism accompany chronic alcohol exposure and diabetes, and lead to neurodegenerative and depressive symptoms (Benton \& Donohoe, 1999; Bettendorff et al., 2014; Abdou \& Hazell, 2015). Thiamine, a water-soluble vitamin B1, plays an important role in cells structure and function and functions as a coenzyme for the $\alpha$-ketoglutarate dehydrogenase complex and pyruvate dehydrogenase, two key mitochondrial enzymes involved in glucose metabolism and Krebs cycle (Micheau et al., 1985; Zhao et al., 2014; Frank, et al., 2015). In our work, we studied whether administration of thiamine or benfotiamine (200 $\mathrm{mg} / \mathrm{kg} /$ day, p.o.) could alter GSK3 3 -dependent tasks such as the acquisition of memory, depressive-like behaviour and the stress response.

In our experiments, two-week administration of thiamine or benfotiamine has precluded an increase of floating behaviour in mice during the last session of the modified forced swim test, similar to imipramine (Chapter 5). Both compounds prevented abnormal activation of GSK3 $\beta$ (Chapter 5). In line with these results, chronic administration of thiamine produced antidepressant effect in a clinic, while, the dose used in the clinical study (300 mg/day) was substantially lower than that used in the present work (Ghaleiha et al., 2016). However, to our knowledge, this is the first demonstration of such effects of these compounds in naïve mice. Also, our studies were the first that identified the role of GSK3 $\beta$ in these effects. 
Thus, the present study suggests the use of thiamine supplementation therapy in depressed patients in support to previously reported observations (Smidt et al., 1991; Benton and Donohoe, 1999; Ghaleiha et al., 2016). In addition, our work is the first demonstration of antidepressant properties of benfotiamine.

An outlook for further experiments to address the role of contextual conditioning in depression: studies with repeated testing in the tail suspension test

In order to test whether delayed re-exposure to adverse context can potentiate depressive-like behaviour under different experimental conditions, we used the tail suspension model, a "dry version" of the forced swim test. Like the forced swim test, the tail suspension model is based on the induction of helplessness and despair behaviour in rodents, which are placed in an inescapable stressful situation for a few minutes (Steru et al., 1985). Both paradigms are regarded to involve similar neurobiological factors and show an overlap in the pharmacological sensitivity to various drugs (Cryan et al., 2005; Costa-Nunes et al., 2015). We have carried out the tail suspension experiment, in which we have used a similar design of the study to employed modified swim test. Namely, mice were exposed to a two-day tail suspension testing in a 6 -min protocol and then additionally were tested on the 5th Day of the experiment. Brains of experimental mice were dissected $10 \mathrm{~min}$ after the last session, hippocampus and prefrontal cortex were isolated and analyzed for GSK3 $\beta$ expression. In these studies we found a strong tendency to a significant increase of a depressive-like behaviour, measured by the total immobility time, on Day 5 (Fig. 7A). In addition, a strong tendency to a significant increase in GSK3 $\beta$ expression in the prefrontal cortex was revealed, and a trend to a positive correlation between the duration of immobility and GSK3 $\beta$ mRNA levels in this brain structure were shown (Fig. 7B). 

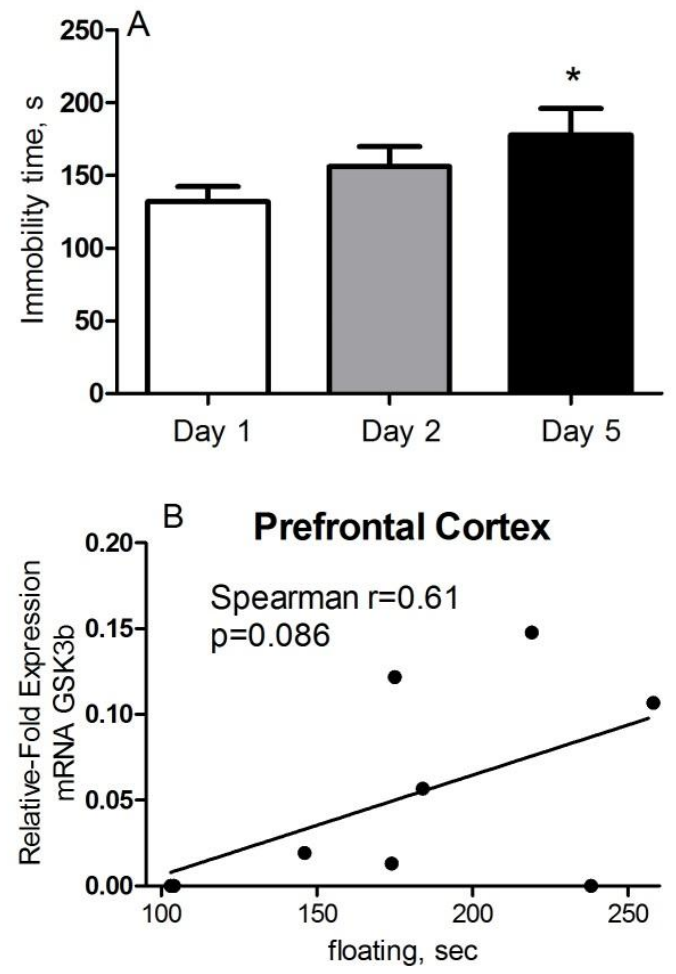

Figure 7. Immobility and gene expression of GSK3b in mice exposed to modified tail suspension test. (A) An increase of the total duration of immobility behaviour in the modified tail suspension test with a delayed session at the Day 5 and (B) correlational analysis of brain GSK3 $\beta$ mRNA and total duration of immobility in the modified tail suspension test with a delayed session at the Day 5. Bars are Means \pm SEMs. Repeated measures ANOVA and Spearman correlation.

Thus, both behavioural and molecular data in the study with modified tail suspension test were overly similar to the findings reported for the modified swim paradigm of comparable design. We believe that an increase of a group sizes in presented pilot experiment with modified tail suspension model, in which seven animals per group were tested, to the range of those used in reported work with modified swim test, would likely allow reach a level of statistical significance in behavioural and molecular assays. This is 
planned to be done in the future studies with modified tail suspension paradigm. In general, the outcome from this experiment suggests a universal nature of changes reported in behavioural and molecular assays with the modified swim study, on which repeated delayed re-exposure to adverse experience was applied.

\section{References}

Abdou, E., Hazell, A.S., 2015. Thiamine deficiency: an update of pathophysiologic mechanisms and future therapeutic considerations. Neurochem. Res. 40, 353-61.

Ahi, J., Radulovic, J., Spiess, J., 2004. The role of hippocampal signaling cascades in consolidation of fear memory. Behav. Brain Res. 149, 17-31.

Ahnaou, A., Drinkenburg, W.H.I.M., 2016. Simultaneous Changes in Sleep, qEEG, Physiology, Behaviour and Neurochemistry in Rats Exposed to Repeated Social Defeat Stress. Neuropsychobiology 73, 209-23.

Amat, J., Baratta, M. V, Paul, E., Bland, S.T., Watkins, L.R., Maier, S.F., 2005. Medial prefrontal cortex determines how stressor controllability affects behaviour and dorsal raphe nucleus. Nat. Neurosci. 8, 365-71.

Ampuero, E., Luarte, A., Santibañez, M., Varas-Godoy, M., Toledo, J., DiazVeliz, G., Cavada, G., Rubio, F.J., Wyneken, U., 2015. Two Chronic Stress Models Based on Movement Restriction in Rats Respond Selectively to Antidepressant Drugs: Aldolase C As a Potential Biomarker. Int. J. Neuropsychopharmacol. 18, 38-40.

Anagnostaras, S.G., Gale, G.D., Fanselow, M.S., 2001. Hippocampus and contextual fear conditioning: recent controversies and advances. Hippocampus 11, 8-17.

Baganz, N., Horton, R., Martin, K., Holmes, A., Daws, L.C., 2010. Repeated Swim Impairs Serotonin Clearance via a Corticosterone-Sensitive Mechanism: Organic Cation Transporter 3, the Smoking Gun. J. Neurosci. 30, 15185-95. 
Benton, D., Donohoe, R.T., 1999. The effects of nutrients on mood. Public Health Nutr. 2, 403-9.

Bergström, A., Jayatissa, M.N., Mørk, A., Wiborg, O., 2008. Stress sensitivity and resilience in the chronic mild stress rat model of depression; an in situ hybridization study. Brain Res. 1196, 41-52.

Bettendorff, L., Lakaye, B., Kohn, G., Wins, P., 2014. Thiamine triphosphate: a ubiquitous molecule in search of a physiological role. Metab. Brain Dis. 29, 1069-82.

Beurel, E., Grieco, S.F., Jope, R.S., 2015. Glycogen synthase kinase-3 (GSK3): Regulation, actions, and diseases. Pharmacol. Ther. 148, 114-31.

Billings, A.G., Cronkite, R.C., Moos, R.H., 1983. Social-environmental factors in unipolar depression: comparisons of depressed patients and nondepressed controls. J. Abnorm. Psychol. 92, 119-33.

Bliss, T. V, Collingridge, G.L., 1993. A synaptic model of memory: long-term potentiation in the hippocampus. Nature 361, 31-9.

Borsini, F., Meli, A., 1988. Is the forced swimming test a suitable model for revealing antidepressant activity? Psychopharmacology (Berl). 94, 147-60.

Calabrese, F., Molteni, R., Racagni, G., Riva, M.A., 2009. Neuronal plasticity: a link between stress and mood disorders. Psychoneuroendocrinology 34 Suppl 1, 208-16.

Carlson, J.N., Glick, S.D., 1991. Brain laterality as a determinant of susceptibility to depression in an animal model. Brain Res. 550, 324-8.

Chen, S.-K., Tvrdik, P., Peden, E., Cho, S., Wu, S., Spangrude, G., Capecchi, M.R., 2010. Hematopoietic origin of pathological grooming in Hoxb8 mutant mice. Cell 141, 775-85.

Chourbaji, S., Zacher, C., Sanchis-Segura, C., Dormann, C., Vollmayr, B., Gass, P., 2005. Learned helplessness: validity and reliability of depressive-like states in mice. Brain Res. Brain Res. Protoc. 16, 70-8. 
Chrousos, G.P., Gold, P.W., 1992. The concepts of stress and stress system disorders. Overview of physical and behavioural homeostasis. JAMA 267, 1244-52.

Costa-Nunes, J.P., Cline, B.H., Araújo-Correia, M., Valença, A., Markova, N., Dolgov, O., Kubatiev, A., Yeritsyan, N., Steinbusch, H.W.M., Strekalova, T., 2015. Animal Models of Depression and Drug Delivery with Food as an Effective Dosing Method: Evidences from Studies with Celecoxib and Dicholine Succinate. Biomed Res. Int. 2015, 596126-32.

Covington, H.E., Maze, I., LaPlant, Q.C., Vialou, V.F., Ohnishi, Y.N., Berton, O., Fass, D.M., Renthal, W., Rush, A.J., Wu, E.Y., Ghose, S., Krishnan, V., Russo, S.J., Tamminga, C., Haggarty, S.J., Nestler, E.J., 2009. Antidepressant actions of histone deacetylase inhibitors. J. Neurosci. 29, 11451-60.

Cryan, J.F., Mombereau, C., Vassout, A., 2005. The tail suspension test as a model for assessing antidepressant activity: review of pharmacological and genetic studies in mice. Neurosci. Biobehav. Rev. 29, 571-625.

Cryan, J.F., Valentino, R.J., Lucki, I., 2005. Assessing substrates underlying the behavioural effects of antidepressants using the modified rat forced swimming test. Neurosci. Biobehav. Rev. 29, 547-569.

Cui, Z., Wang, H., Tan, Y., Zaia, K.A., Zhang, S., Tsien, J.Z., 2004. Inducible and reversible NR1 knockout reveals crucial role of the NMDA receptor in preserving remote memories in the brain. Neuron 41, 781-93.

Cunha, 2010. A simple role for BDNF in learning and memory? Front. Mol. Neurosci. 9, 3-10.

Dahlhoff, M., Siegmund, A., Golub, Y., Wolf, E., Holsboer, F., Wotjak, C.T., 2010. AKT/GSK3 $\beta /$ beta-catenin signalling within hippocampus and amygdala reflects genetically determined differences in post-traumatic stress disorder like symptoms. Neuroscience 169, 1216-26.

De Boer, S.F., Slangen, J.L., Van der Gugten, J., 1990. Plasma catecholamine and corticosterone levels during active and passive shock-prod avoidance 
behaviour in rats: Effects of chlordiazepoxide. Physiol. Behav. 47, 10891098.

De Pablo, J.M., Parra, A., Segovia, S., Guillamón, A., 1989. Learned immobility explains the behaviour of rats in the forced swimming test. Physiol. Behav. 46, 229-37.

Diniz, B.S., Talib, L.L., Giroud Joaquim, H.P., de Paula, V.R.J., Gattaz, W.F., Forlenza, O.V., 2011. Platelet GSK3B activity in patients with late-life depression: Marker of depressive episode severity and cognitive impairment? World J. Biol. Psychiatry 12, 216-22.

Dudai, Y., 2012. The restless engram: consolidations never end. Annu. Rev. Neurosci. 35, 227-47.

Elgarf, A.S.A., Aboul-Fotouh, S., Abd-Alkhalek, H.A., El Tabbal, M., Hassan, A.N., Kassim, S.K., Hammouda, G.A., Farrag, K.A., Abdel-Tawab, A.M., 2014. Lipopolysaccharide repeated challenge followed by chronic mild stress protocol introduces a combined model of depression in rats: Reversibility by imipramine and pentoxifylline. Pharmacol. Biochem. Behav. 126, 152-62.

Etkin, A., 2010. Functional neuroanatomy of anxiety: a neural circuit perspective. Curr. Top. Behav. Neurosci. 2, 251-77.

Farhang, S., Barar, J., Fakhari, A., Mesgariabbasi, M., Khani, S., Omidi, Y., Farnam, A., 2014. Asymmetrical expression of BDNF and NTRK3 genes in frontoparietal cortex of stress-resilient rats in an animal model of depression. Synapse 68, 387-93.

Flores, Á., Valls-Comamala, V., Costa, G., Saravia, R., Maldonado, R., Berrendero, F., 2014. The hypocretin/orexin system mediates the extinction of fear memories. Neuropsychopharmacology 39, 2732-41.

Frank, L.L., 2015. Thiamin in Clinical Practice. J. Parenter. Enter. Nutr. 39, 503-20.

Frankland, P.W., Bontempi, B., 2005. The organization of recent and remote memories. Nat. Rev. Neurosci. 6, 119-30. 
Frankland, P.W., Bontempi, B., Talton, L.E., Kaczmarek, L., Silva, A.J., 2004. The involvement of the anterior cingulate cortex in remote contextual fear memory. Science 304, 881-3.

Ghaleiha, A., Davari, H., Jahangard, L., Haghighi, M., Ahmadpanah, M., Seifrabie, M.A., Bajoghli, H., Holsboer-Trachsler, E., Brand, S., 2016. Adjuvant thiamine improved standard treatment in patients with major depressive disorder: results from a randomized, double-blind, and placebo-controlled clinical trial. Eur. Arch. Psychiatry Clin. Neurosci. 1-8.

Giustino, T.F., Maren, S., 2015. The Role of the Medial Prefrontal Cortex in the Conditioning and Extinction of Fear. Front. Behav. Neurosci. 9, 298-311.

Gold, P.E., Korol, D.L., 2012. Making memories matter. Front. Integr. Neurosci. 6, 116.

Gorka, A.X., LaBar, K.S., Hariri, A.R., 2016. Variability in emotional responsiveness and coping style during active avoidance as a window onto psychological vulnerability to stress. Physiol. Behav. 158, 90-9.

Gould, T.D., O'Donnell, K.C., Picchini, A.M., Dow, E.R., Chen, G., Manji, H.K., 2008. Generation and behavioural characterization of beta-catenin forebrain-specific conditional knock-out mice. Behav. Brain Res. 189, 11725.

Grimes, C.A., Jope, R.S., 2001. The multifaceted roles of glycogen synthase kinase 3beta in cellular signaling. Prog. Neurobiol. 65, 391-426.

Harro, J., Kanarik, M., Matrov, D., Panksepp, J., 2011. Mapping patterns of depression-related brain regions with cytochrome oxidase histochemistry: relevance of animal affective systems to human disorders, with a focus on resilience to adverse events. Neurosci. Biobehav. Rev. 35, 1876-89.

Hauer, D., Weis, F., Papassotiropoulos, A., Schmoeckel, M., BeirasFernandez, A., Lieke, J., Kaufmann, I., Kirchhoff, F., Vogeser, M., Roozendaal, B., Briegel, J., de Quervain, D., Schelling, G., 2011. Relationship of a common polymorphism of the glucocorticoid receptor gene to traumatic memories 
and post-traumatic stress disorder in patients after intensive care therapy. Crit. Care Med. 39, 643-50.

Havranek, M.M., Bolliger, B., Roos, S., Pryce, C.R., Quednow, B.B., Seifritz, E., 2016. Uncontrollable and unpredictable stress interacts with subclinical depression and anxiety scores in determining anxiety response. Stress 19, 53-62.

Higuchi, F., Uchida, S., Yamagata, H., Abe-Higuchi, N., Hobara, T., Hara, K., Kobayashi, A., Shintaku, T., Itoh, Y., Suzuki, T., Watanabe, Y., 2016. Hippocampal MicroRNA-124 Enhances Chronic Stress Resilience in Mice. J. Neurosci. 36, 7253-67.

Holsboer, F., 2000. The corticosteroid receptor hypothesis of depression. Neuropsychopharmacology 23, 477-501.

Holtzheimer, P.E., Mayberg, H.S., 2011. Deep brain stimulation for psychiatric disorders. Annu. Rev. Neurosci. 34, 289-307.

Jiang, Y., Jakovcevski, M., Bharadwaj, R., Connor, C., Schroeder, F.A., Lin, C.L., Straubhaar, J., Martin, G., Akbarian, S., 2010. Setdb1 histone methyltransferase regulates mood-related behaviours and expression of the NMDA receptor subunit NR2B. J. Neurosci. 30, 7152-67.

Job, R.F., Barnes, B.W., 1995. Stress and consumption: inescapable shock, neophobia, and quinine finickiness in rats. Behav. Neurosci. 109, 106-16.

Johansen, J.P., Cain, C.K., Ostroff, L.E., LeDoux, J.E., 2011. Molecular mechanisms of fear learning and memory. Cell 147, 509-24.

Jope, R.S., Roh, M.-S., 2006. Glycogen synthase kinase-3 (GSK3) in psychiatric diseases and therapeutic interventions. Curr. Drug Targets 7, 1421-34.

Jurado-Arjona, J., Llorens-Martín, M., Ávila, J., Hernández, F., 2016. GSK3 $\beta$ Overexpression in Dentate Gyrus Neural Precursor Cells Expands the Progenitor Pool and Enhances Memory Skills. J. Biol. Chem. 291, 8199-213.

Karege, F., Perroud, N., Burkhardt, S., Schwald, M., Ballmann, E., La Harpe, R., Malafosse, A., 2007. Alteration in kinase activity but not in protein levels 
of protein kinase B and glycogen synthase kinase-3beta in ventral prefrontal cortex of depressed suicide victims. Biol. Psychiatry 61, 240-5.

Katche, C., Cammarota, M., Medina, J.H., 2013. Molecular signatures and mechanisms of long-lasting memory consolidation and storage. Neurobiol. Learn. Mem. 106, 40-7.

Kim, J.J., Fanselow, M.S., 1992. Modality-specific retrograde amnesia of fear. Science 256, 675-7.

Kim, Y., Perova, Z., Mirrione, M.M., Pradhan, K., Henn, F.A., Shea, S., Osten, P., Li, B., 2016. Whole-Brain Mapping of Neuronal Activity in the Learned Helplessness Model of Depression. Front. Neural Circuits 10, 40-7.

Kimura, T., Yamashita, S., Nakao, S., Park, J.M., Murayama, M., Mizoroki, T., Yoshiike, Y., Sahara, N., Takashima, A., 2008. GSK3 $\beta$ is required for memory reconsolidation in adult brain. PLoS One 3, 110-7.

Kozlovsky, N., Matar, M.A., Kaplan, Z., Kotler, M., Zohar, J., Cohen, H., 2007. Long-term down-regulation of BDNF mRNA in rat hippocampal CA1 subregion correlates with PTSD-like behavioural stress response. Int. J. Neuropsychopharmacol. 10, 741-58.

Krahe, T.E., Filgueiras, C.C., Schmidt, S.L., 2002. Effects of rotational side preferences on immobile behaviour of normal mice in the forced swimming test. Prog. Neuropsychopharmacol. Biol. Psychiatry 26, 169-76.

Kriaucionis, S., Heintz, N., 2009. The nuclear DNA base 5hydroxymethylcytosine is present in Purkinje neurons and the brain. Science 324, 929-30.

Krishnan, V., Han, M.H., Graham, D.L., Berton, O., Renthal, W., Russo, S.J., LaPlant, Q., Graham, A., Lutter, M., Lagace, D.C., Ghose, S., Reister, R., Tannous, P., Green, T.A., Neve, R.L., Chakravarty, S., Kumar, A., Eisch, A.J., Self, D.W., Lee, F.S., Tamminga, C.A., Cooper, D.C., Gershenfeld, H.K., Nestler, E.J., 2007. Molecular Adaptations Underlying Susceptibility and Resistance to Social Defeat in Brain Reward Regions. Cell 131, 391-404. 
Lesch, K.P., Mössner, R., 2006. Inactivation of 5HT transport in mice: modeling altered 5HT homeostasis implicated in emotional dysfunction, affective disorders, and somatic syndromes. Handb. Exp. Pharmacol. 1, 41756.

Li, G., Liu, T., Kong, X., Wang, L., Jin, X., 2014. Hippocampal glycogen synthase kinase $3 \beta$ is critical for the antidepressant effect of cyclindependent kinase 5 inhibitor in rats. J. Mol. Neurosci. 54, 92-99.

Liu, X.S., Xue, Q. sheng, Zeng, Q.W., Li, Q., Liu, J., Feng, X.M., Yu, B.W., 2010. Sevoflurane impairs memory consolidation in rats, possibly through inhibiting phosphorylation of glycogen synthase kinase-3beta in the hippocampus. Neurobiol. Learn. Mem. 94, 461-67.

Lolak, S., Suwannarat, P., Lipsky, R.H., 2014. Epigenetics of depression. Prog. Mol. Biol. Transl. Sci. 128, 103-37.

Maguschak, K.A., Ressler, K.J., 2008. Beta-catenin is required for memory consolidation. Nat. Neurosci. 11, 1319-26.

Maren, S., Phan, K.L., Liberzon, I., 2013. The contextual brain: implications for fear conditioning, extinction and psychopathology. Nat. Rev. Neurosci. 14, 417-28.

Martin, S.J., Grimwood, P.D., Morris, R.G., 2000. Synaptic plasticity and memory: an evaluation of the hypothesis. Annu. Rev. Neurosci. 23, 649-711.

Mayberg, H.S., Liotti, M., Brannan, S.K., McGinnis, S., Mahurin, R.K., Jerabek, P.A., Silva, J.A., Tekell, J.L., Martin, C.C., Lancaster, J.L., Fox, P.T., 1999. Reciprocal limbic-cortical function and negative mood: converging PET findings in depression and normal sadness. Am. J. Psychiatry 156, 675-82.

McArthur, R., Borsini, F., 2006. Animal models of depression in drug discovery: a historical perspective. Pharmacol. Biochem. Behav. 84, 436-52.

McKinney, W.T., Bunney, W.E., 1969. Animal model of depression. I. Review of evidence: implications for research. Arch. Gen. Psychiatry 21, 240-8. 
Micheau, J., Durkin, T.P., Destrade, C., Rolland, Y., Jaffard, R., 1985. Chronic administration of sulbutiamine improves long term memory formation in mice: Possible cholinergic mediation. Pharmacol. Biochem. Behav. 23, 1958.

Miller, A.M.P., Vedder, L.C., Law, L.M., Smith, D.M., 2014. Cues, context, and long-term memory: the role of the retrosplenial cortex in spatial cognition. Front. Hum. Neurosci. 8, 5-13.

Monestier, C., Gilot-Fromont, E., Morellet, N., Debeffe, L., Cebe, N., Merlet, J., Picot, D., Rames, J.-L., Hewison, A.J.M., Verheyden, H., 2016. Individual variation in an acute stress response reflects divergent coping strategies in a large herbivore. Behav. Processes 132, 22-8.

Montes, P., Ruiz-Sánchez, E., Calvillo, M., Rojas, P., 2016. Active coping of prenatally stressed rats in the forced swimming test: involvement of the Nurr1 gene. Stress 19, 506-15.

Natelson, B.H., Ottenweller, J.E., Cook, J.A., Pitman, D., McCarty, R., Tapp, W.N., 1988. Effect of stressor intensity on habituation of the adrenocortical stress response. Physiol. Behav. 43, 41-6.

Nemeroff, C.B., Bremner, J.D., Foa, E.B., Mayberg, H.S., North, C.S., Stein, M.B., 2006. Post-traumatic stress disorder: A state-of-the-science review. J. Psychiatr. Res. 40, 1-21.

Oh, D.H., Park, Y.C., Kim, S.H., 2010. Increased glycogen synthase kinase-3 $\beta$ mRNA level in the hippocampus of patients with major depression: a study using the stanley neuropathology consortium integrative database. Psychiatry Investig. 7, 202-7.

Omata, N., Chiu, C.-T., Moya, P.R., Leng, Y., Wang, Z., Hunsberger, J.G., Leeds, P., Chuang, D.-M., 2011. Lentivirally mediated GSK3 $\beta$ silencing in the hippocampal dentate gyrus induces antidepressant-like effects in stressed mice. Int. J. Neuropsychopharmacol. 14, 711-7.

Overstreet, D.H., 2012. Modeling depression in animal models. Methods Mol. Biol. 829, 125-44. 
Pan, X., Gong, N., Zhao, J., Yu, Z., Gu, F., Chen, J., Sun, X., Zhao, L., Yu, M., Xu, Z., Dong, W., Qin, Y., Fei, G., Zhong, C., Xu, T. Le, 2010. Powerful beneficial effects of benfotiamine on cognitive impairment and $\beta$-amyloid deposition in amyloid precursor protein/presenilin-1 transgenic mice. Brain 133, 1342-51.

Peña, C.J., Bagot, R.C., Labonté, B., Nestler, E.J., 2014. Epigenetic signaling in psychiatric disorders. J. Mol. Biol. 426, 3389-412.

Perrault, G., Morel, E., Zivkovic, B., Sanger, D.J., 1992. Activity of litoxetine and other serotonin uptake inhibitors in the tail suspension test in mice. Pharmacol. Biochem. Behav. 42, 45-7.

Phillips, R.G., LeDoux, J.E., n.d. Lesions of the dorsal hippocampal formation interfere with background but not foreground contextual fear conditioning. Learn. Mem. 1, 34-44.

Polter, A., Beurel, E., Yang, S., Garner, R., Song, L., Miller, C.A., Sweatt, J.D., McMahon, L., Bartolucci, A.A., Li, X., Jope, R.S., 2010. Deficiency in the Inhibitory Serine-Phosphorylation of Glycogen Synthase Kinase-3 Increases Sensitivity to Mood Disturbances. Neuropsychopharmacology. 35, 1761-74

Ponimaskin, E., Dityateva, G., Ruonala, M.O., Fukata, M., Fukata, Y., Kobe, F., Wouters, F.S., Delling, M., Bredt, D.S., Schachner, M., Dityatev, A., 2008. Fibroblast growth factor-regulated palmitoylation of the neural cell adhesion molecule determines neuronal morphogenesis. J. Neurosci. 28, 8897-907.

Possamai, F., dos Santos, J., Walber, T., Marcon, J.C., dos Santos, T.S., Lino de Oliveira, C., 2014. Influence of enrichment on behavioural and neurogenic effects of antidepressants in Wistar rats submitted to repeated forced swim test. Prog. Neuro-Psychopharmacology Biol. Psychiatry 58, 15-21.

Raabe, F.J., Spengler, D., 2013. Epigenetic Risk Factors in PTSD and Depression. Front. psychiatry 4, 80-5.

Reinelt, E., Barnow, S., Stopsack, M., Aldinger, M., Schmidt, C.O., John, U., Grabe, H.J., 2015. Social support and the serotonin transporter genotype (5HTTLPR) moderate levels of resilience, sense of coherence, and depression. Am. J. Med. Genet. Part B Neuropsychiatr. Genet. 168, 383-91. 
Rogóz, Z., Skuza, G., Legutko, B., 2007. Repeated co-treatment with imipramine and amantadine induces hippocampal brain-derived neurotrophic factor gene expression in rats. J. Physiol. Pharmacol. 58, 21934.

Rogóz, Z., Skuza, G., Wójcikowski, J., Daniel, W.A., 2003. Effects of combined treatment with imipramine and metyrapone in the forced swimming test in rats. Behavioural and pharmacokinetic studies. Pol. J. Pharmacol. 55, 993-9.

Ronai, Z., Kovacs-Nagy, R., Szantai, E., Elek, Z., Sasvari-Szekely, M., Faludi, G., Benkovits, J., Rethelyi, J.M., Szekely, A., 2014. Glycogen synthase kinase 3 beta gene structural variants as possible risk factors of bipolar depression. Am. J. Med. Genet. Part B Neuropsychiatr. Genet. 165, 217-22.

Rozeske, R.R., Valerio, S., Chaudun, F., Herry, C., 2015. Prefrontal neuronal circuits of contextual fear conditioning. Genes, Brain Behav. 14, 22-36.

Rudy, J.W., 2009. Context representations, context functions, and the parahippocampal-hippocampal system. Learn. Mem. 16, 573-85.

Saus, E., Soria, V., Escaramís, G., Crespo, J.M., Valero, J., Gutiérrez-Zotes, A., Martorell, L., Vilella, E., Menchón, J.M., Estivill, X., Gratacòs, M., Urretavizcaya, M., 2010. A haplotype of glycogen synthase kinase $3 \beta$ is associated with early onset of unipolar major depression. Genes. Brain. Behav. 9, 799-807.

Seligman, M.E., Beagley, G., 1975. Learned helplessness in the rat. J. Comp. Physiol. Psychol. 88, 534-41.

Silva, R., Mesquita, A.R., Bessa, J., Sousa, J.C., Sotiropoulos, I., Leão, P., Almeida, O.F.X., Sousa, N., 2008. Lithium blocks stress-induced changes in depressive-like behaviour and hippocampal cell fate: The role of glycogensynthase-kinase-3 $\beta$. Neuroscience 152, 656-69.

Sintoni, S., Kurtys, E., Scandaglia, M., Contestabile, A., Monti, B., 2013. Chronic valproic acid administration impairs contextual memory and dysregulates hippocampal GSK3 $\beta$ in rats. Pharmacol. Biochem. Behav. 106, 8-15. 
Smidt, L.J., Cremin, F.M., Grivetti, L.E., Clifford, A.J., 1991. Influence of thiamin supplementation on the health and general well-being of an elderly Irish population with marginal thiamin deficiency. J. Gerontol. 46, 16-22.

Sotres-Bayon, F., Quirk, G.J., 2010. Prefrontal control of fear: more than just extinction. Curr. Opin. Neurobiol. 20, 231-5.

Steru, L., Chermat, R., Thierry, B., Simon, P., 1985. The tail suspension test: a new method for screening antidepressants in mice. Psychopharmacology (Berl). 85, 367-70.

Strekalova, T., Couch, Y., Kholod, N., Boyks, M., Malin, D., Leprince, P., Steinbusch, H.M., 2011. Update in the methodology of the chronic stress paradigm: internal control matters. Behav. Brain Funct. 7, 9-18.

Strekalova, T., Spanagel, R., Bartsch, D., Henn, F.A., Gass, P., 2004. Stressinduced anhedonia in mice is associated with deficits in forced swimming and exploration. Neuropsychopharmacology 29, 2007-17.

Strekalova, T., Sun, M., Sibbe, M., Evers, M., Dityatev, A., Gass, P., Schachner, $M ., 2002$. Fibronectin domains of extracellular matrix molecule tenascin-C modulate hippocampal learning and synaptic plasticity. Mol. Cell. Neurosci. 21, 173-87.

Strekalova, T., Wotjak, C.T., Schachner, M., 2001. Intrahippocampal administration of an antibody against the HNK-1 carbohydrate impairs memory consolidation in an inhibitory learning task in mice. Mol. Cell. Neurosci. 17, 1102-13.

Strekalova, T. V, 1995. The characteristics of the defensive behaviour of rats in accordance with their resistance to emotional stress. Zh. Vyssh. Nerv. Deiat. Im. I P Pavlova 45, 420-2.

Sutherland, R.J., Sparks, F.T., Lehmann, H., 2010. Hippocampus and retrograde amnesia in the rat model: a modest proposal for the situation of systems consolidation. Neuropsychologia 48, 2357-69. 
Suzuki, M.M., Bird, A., 2008. DNA methylation landscapes: provocative insights from epigenomics. Nat. Rev. Genet. 9, 465-76.

Taché, Y., 2015. Corticotrophin-releasing factor 1 activation in the central amygdale and visceral hyperalgesia. Neurogastroenterol. Motil. 27, 1-6.

Tohda, M., Mingmalairak, S., Murakami, Y., Matsumoto, K., 2010. Enhanced expression of BCL2/adenovirus EIB 19-kDa-interacting protein 3 mRNA, a candidate for intrinsic depression-related factor, and effects of imipramine in the frontal cortex of stressed mice. Biol. Pharm. Bull. 33, 53-7.

Tsankova, N.M., Berton, O., Renthal, W., Kumar, A., Neve, R.L., Nestler, E.J., 2006. Sustained hippocampal chromatin regulation in a mouse model of depression and antidepressant action. Nat. Neurosci. 9, 519-25.

Uchida, S., Hara, K., Kobayashi, A., Otsuki, K., Yamagata, H., Hobara, T., Suzuki, T., Miyata, N., Watanabe, Y., 2011. Epigenetic status of Gdnf in the ventral striatum determines susceptibility and adaptation to daily stressful events. Neuron 69, 359-72.

Vollmayr, B., Mahlstedt, M.M., Henn, F.A., 2007. Neurogenesis and depression: what animal models tell us about the link. Eur. Arch. Psychiatry Clin. Neurosci. 257, 300-3.

Warden, M.R., Selimbeyoglu, A., Mirzabekov, J.J., Lo, M., Thompson, K.R., Kim, S.-Y., Adhikari, A., Tye, K.M., Frank, L.M., Deisseroth, K., 2012. A prefrontal cortex-brainstem neuronal projection that controls response to behavioural challenge. Nature 492, 428-32.

Weaver, I.C.G., Cervoni, N., Champagne, F.A., D’Alessio, A.C., Sharma, S., Seckl, J.R., Dymov, S., Szyf, M., Meaney, M.J., 2004. Epigenetic programming by maternal behaviour. Nat. Neurosci. 7, 847-54.

Wiborg, O., 2013. Chronic mild stress for modeling anhedonia. Cell Tissue Res. 354, 155-69.

Wilkinson, M.B., Dias, C., Magida, J., Mazei-Robison, M., Lobo, M., Kennedy, P., Dietz, D., Covington, H., Russo, S., Neve, R., Ghose, S., Tamminga, C., 
Nestler, E.J., 2011. A novel role of the WNT-dishevelled-GSK3 $\beta$ signaling cascade in the mouse nucleus accumbens in a social defeat model of depression. J. Neurosci. 31, 9084-92.

Wilkinson, M.B., Xiao, G., Kumar, A., LaPlant, Q., Renthal, W., Sikder, D., Kodadek, T.J., Nestler, E.J., 2009. Imipramine treatment and resiliency exhibit similar chromatin regulation in the mouse nucleus accumbens in depression models. J. Neurosci. 29, 7820-32.

Willner, P., 1984. The validity of animal models of depression. Psychopharmacology (Berl). 83, 1-16.

Willner, P., Belzung, C., 2015. Treatment-resistant depression: are animal models of depression fit for purpose? Psychopharmacology (Berl). 232, 3473-95.

Wu, P., Xue, Y.-X., Ding, Z.-B., Xue, L.-F., Xu, C.-M., Lu, L., 2011. Glycogen synthase kinase $3 \beta$ in the basolateral amygdala is critical for the reconsolidation of cocaine reward memory. J. Neurochem. 118, 113-25.

Wüst, S., Federenko, I.S., van Rossum, E.F.C., Koper, J.W., Hellhammer, D.H., 2005. Habituation of cortisol responses to repeated psychosocial stressfurther characterization and impact of genetic factors. Psychoneuroendocrinology 30, 199-211.

Yizhar, O., Fenno, L.E., Davidson, T.J., Mogri, M., Deisseroth, K., 2011. Optogenetics in neural systems. Neuron 71, 9-34.

Zacharko, R.M., Anisman, H., 1991. Stressor-induced anhedonia in the mesocorticolimbic system. Neurosci. Biobehav. Rev. 15, 391-405.

Zhang, R.-X., Han, Y., Chen, C., Xu, L.-Z., Li, J.-L., Chen, N., Sun, C.-Y., Chen, W.-H., Zhu, W.-L., Shi, J., Lu, L., 2016. EphB2 in the Medial Prefrontal Cortex Regulates Vulnerability to Stress. Neuropsychopharmacology 41, 2541-56.

Zhao, Y., Wu, Y., Hu, H., Cai, J., Ning, M., Ni, X., Zhong, C., 2014. Downregulation of transketolase activity is related to inhibition of 
hippocampal progenitor cell proliferation induced by thiamine deficiency. Biomed Res. Int. 2014, 5-16. 


\section{Summary}

Our study with 3,5-diiodo-L-thyronine (T2), a thyroid hormone that was earlier regarded functionally inactive, has revealed its effects in the two-day tail suspension test. In this model, a bolus injection of $\mathrm{T} 2$ at two different doses, to mice of two different strains, resulted in a decrease of depressivelike behaviour, thus, suggesting the antidepressant-like effect of T2 (Chapter 2). This effect of T2 was not known before; it suggested clinical use of $T 2$ under conditions that require faster than with other thyroid hormones therapeutic effect. However, no effects of $\mathrm{T} 2$ on depressive-like behaviour were found in a classic two-day forced swim test (Chapter 3). Thus, our results have demonstrated a limited sensitivity of the forced swim test that is in line with available literature, and suggested a need for its modification so the accuracy of the test could be increased.

In this context, we modified commonly used protocol of the forced swim test, where an additional session on Day 5 has followed the initial exposure on Days 1 and 2 in its classical variant (Chapter 4). We hypothesized that additional delayed session on Day 5 can model increased contextual learning and consolidation of environmental adversities related to the testing. Increased brain expression of GSK3 $\beta$, a hallmark of a distress and depression in humans was observed in mice after modified, but not classic sessions of the swim test (Chapter 4). Moreover, increased scores of depressive-like behaviour (floating behaviour) during additional delayed testing positively correlated with the levels of GSK3 $\beta$ mRNA in the prefrontal cortex of experimental groups (Chapter 4). No such correlations were found in the hippocampal formation, while GSK3 $\beta$ expression was increased there as well. Elevated activities of GSK3 $\beta$ during repeated but not single exposure to swimming were further demonstrated on a protein level. The concentration of 9-Ser-phosphorilated (inactive) form of pS9-GSK3 $\beta$ and its ratio to total GSK3 $\beta$ were significantly decreased during repeated testing (Chapter 4).

Our experiments have identified the role of timing and exposure to a context in the development of behavioural and molecular changes during the modified swim test. A "premature" exposure to swimming on Day 3 instead 
of Day 5 did not result in expression of GSK3 $\beta$ (Chapter 4). On other hand, a replacement of a swim session with an exposure to the context of testing on Day 2 and Day 5 or the omission of intermediate swimming or context exposure on these days affected floating behaviour and brain GSK3 $\beta$ mRNA in the modified swim test (Chapter 4). Together, our experiments suggested the critical role of GSK3 $\beta$ in the "consolidation phase" of the development depressive-like features associated with enhanced conditioning to adverse context.

Two-week delivery of low dose of imipramine $(7.5 \mathrm{mg} / \mathrm{kg} /$ day $)$, thiamine and benfotiamine ( $200 \mathrm{mg} / \mathrm{kg} / \mathrm{day}$ ) has precluded an increase of floating and over-expression of in mice during the last session of the modified forced swim test (Chapter 5). Given that all these compounds were shown to decrease GSK3 $\beta$ activities, these data further suggest the role of this molecular factor in enhanced contextual conditioning associated with depressive-like state. In addition, these data have identified thiamine and its precursor as antidepressant agents and suggested their usefulness in treatment of depression in clinic (Chapter 5). 


\section{Valorization}

Relevance for society

Major depression was projected to become the second most common cause of disability worldwide by the next decade. Depression as a major health issue is illustrated by its death-toll, which currently claims more lives per year than road-traffic accidents. At the same time, there is an obvious need for an improvement in the treatment of depression, as up to $45 \%$ of depressed patients do not show improved mood after advanced therapy and $15 \%$ of patients do not respond to any antidepressant therapies. Important features of depression are its link to stress response and exaggerated cognitive processing of negative experiences; the neurobiology of these phenomena, however, was not properly addressed in most of available animal models of this disorder. The complexity of the mechanisms of depression and a lack of reliable and effective methods of modeling depression in animals complicate the search for more effective treatments of this disorder.

As and outcome from our studies, we have offered a new model of depression, which can mimic behavioural and molecular signs of a depressive-like state, such as helpless behaviour and the over-expression of GSK3 $\beta$, a molecular hub of distress/major depression in humans. This new mouse paradigm models a new aspect of depression that was not addressed so far in translational research with this condition, an enhanced contextual conditioning of adversity. In addition, our mouse model replicates an interindividual variability in the susceptibility to depressive disorder and thus, can enable the studies of the neurobiology of distinct susceptibility to depressive syndrome that is rarely possible in pre-clinical modeling of depression.

Importantly, newly proposed modified swim test with delayed testing requires minimal experimental work (three sessions of $6 \mathrm{~min}$ each) that is highly relevant for animal welfare and labour costs. In comparison to basic models of depressive behaviours such as Porsolt test and tail suspension test, our model provides more refined way of modeling a depressive-like state in small rodents that is also highly economically effective. Other models of comparably high face and construct validity in modelling 
depression, as for instance, chronic stress and learned helplessness paradigm, require extremely high labour and time costs and regarded as not optimal in terms of animals well-being. In general, we believe that the modified swim test may provide a methodological solution for combining the advantages of chronic models of depression, while overcoming their disadvantages of highly demanding resources they typically require, as well as improving laboratory animal welfare. Given listed above advantages of the model, we trust that this paradigm can be very useful in screening of new drug candidates against depression.

We believe that our studies that have identified antidepressant-like properties of thiamine and its highly bioavailable precursor benfotiamine that were not known before, support this view and demonstrate a power of new modified forced swim test. Our studies demonstrated that chronic applications for the thiamine (vitamin B1) and its precursor benfotiamine reduce molecular and behavioural changes associated with depression that suggests their use as supplementary therapy. Also, we found that the doses of imipramine which are much lower than commonly used concentrations of this antidepressant are efficient in preventing depressive-like changes. This can help to refine the dose of tricyclics in the clinical practice. Finally, our experiments with T2, previously regarded as inactive form pf thyroid hormone, revealed its antidepressant-like properties. Thus, T2 can be used to counteract depressive symptoms as well.

\section{Target groups}

We consider our target groups could be patients and individuals, who are genetically susceptible to develop depression or exert clinical depression, which is particularly associated with experience of traumatic events. Also, care takers who can suffer from their activities related to these individual can be considered as target groups.

\section{Activity / Products}

As potential outlook of presented work, we foresee a potential interest of pharmaceutical companies to complete necessary tests and develop thiamine alone with its precursors, and T2 hormone as formulations for 
pharmacotherapy of depression and associated symptoms that would help to improve a public health.

In addition, in a course of the present Thesis we developed the modified paradigm that offers more effective and refined approach to address the role of enhanced cognitive processing in the pathophysiological mechanisms of depression. This can result in the identification of new targets of pharmacological management of depression and screen new candidates to antidepressant therapies more accurately and effectively.

\section{Innovation}

The work hereby presented has been innovative in various regards. First, we have established and validated a new mouse depression paradigm; so far, a few, if any analogues of it were reported in the literature. Second, with this paradigm, one can segregate susceptible versus resilient to depressive state animals at very low labour and time costs that was not possible earlier. Third, this is the first depression model that enables the tracking of the dynamics of molecular / neurobiological substrate of this condition in relation to the time of a trigger of a depressive-like state; no such paradigms were reported in the literature till now. Fourth, molecular and neuroanatomical substrates of enhanced contextual conditioning have been determined, such as an over-expression of GSK3 $\beta$ in the prefrontal cortex. Finally, T2 and thiamine (vitamin B1) were identified as new therapies of depressive state that was unknown before.

\section{Implementation}

In line with the above mentioned relevance for the scientific and medical communities, society and industry, the implementation of the knowledge generated in the current dissertation is also multidimensional, as discussed above. From an academic perspective, results have been or will be published in peer-reviewed international journals and presented at national and international conferences. 


\section{Acknowledgments}

First of all, I would like to express my gratitude to Professor Harry Steinbusch for general supervision of joint research project between Maastricht University, Department of Neuroscience and Institute of Physiologically Active Compounds (IPAC) of Russian Academy of Sciences. Professor Steinbusch had contributed in a long-term collaboration between two Research Labouratories in which I was privileged to take part over the time between 2011 and 2017. This work was supported by International Foundation Alzheimer's Research (ISAO; Internationale Stichting Alzheimer Onderzoek) foundation, "5-100 Russian Excellence Project", Russian Foundation of Basic Research and has culminated in several important publications. Clearly, it would be not possible without irreplaceable contribution of Professor Steinbusch in these contacts.

I would like to express my special gratitude to Dr. Tatyana Strekalova, my immediate supervisor with my Thesis, who coached me across probably the most challenging but also highly rewarding 6-year period of my career between 2011 and 2017. It is her I owe my immense gratitude for the literal opening of the most amazing research and training opportunities for me, which were offered to me under the best international and local standards. In a course of my work under her supervision, I have learned the most refined methods of behavioural techniques, and through her collabourators in the Universities of Oxford, St- Petersburg, Lyon, Wuerzburg and Lisbon, had a chance to acquire other critical techniques in neurosciences for my Thesis. As a part of this intense international training, I was able to be introduced to research environment of the most prominent labouratories in Europe working in the field of Neuroscience. Thanks to a remarkable research and educational commitment of Dr. Tatyana Strekalova, I was able to carry through the challenges of multi-disciplinary research I was involved during my Thesis with the most positive outcome. With her help, I was able to contribute to 7 peer-review papers and present my data at 5 International conferences. I am also much thankful for her encouragement, criticism, innovative scientific and organizational solutions and patience that she expressed towards me over the time. I trust it is quite impossible to 
overestimate the role of $\mathrm{Dr}$ Tatyana Strekalova in my PhD Thesis and my scientific career at this point.

Likewise, I would like to express my sincere gratitude and appreciation to Professor Klaus-Peter Lesch, who provided me a unique opportunity to join his team and let me to be a part of his immediate research environment. Thanks to Professor Lesch, I had a chance to learn not only the basics of the best standards of immunohistochemistry and molecular biology, but also have gain a priceless experience of planning and arranging my work independently and communicate my plans to other colleagues, also in English language. I am especially thankful to Professor Lesch and his family for the most warm welcome in his house and help throughout my DAAD training time in Wuerzburg University.

Next, help and a constant support of Professor Sergey Bachurin in my PhD project are not to be over-estimated. I am very grateful for providing me research facilities via IPAC labs, and constant support and supervision. No doubts, without constant and patient support of Professor Bachurin my publications, defence of this Thesis and entire work would not be possible.

I also would like to say the words of my deep appreciation of the most warm support of Dr. Elena Shevtsova who was not only a scientific co-supervisor of mine, but also provided me with irreplaceable emotional support and advises during my work on the Thesis. I would like to thank of Dr Elena Shevtsova for all stimulating discussions regarding my experiments, data interpretation and for being always in a position of finding time and energy for these and many other questions I would constantly bring to her attention, being in her lab in IPAC.

It is my great pleasure to acknowledge scientific, technical and organizational support of Prof. Daniel Anthony from Oxford University, Dr. Olga Zubareva from the Institute of Experimental Medicine in St Petersburg, Dr. Angelika Schmitt from University of Wuerzburg and Prof. Lucien Bettendorff from University of Liege. I feel privileged having an opportunity to come across your scientific work and activities and would like to thank all of you for your contribution to my training and to my PhD Thesis and 
personal communication. Also, I am very pleased to thank from my heart, Dr. Dmitry Malin from Wisconsin Univesrity, Prof. Andrey Svystunov and Prof. Andrey Zamyatnin from the 1st Sechenov Moscow State Medical University, without whose material and scientific support my Thesis would be likely be not finalized. I am much grateful to my colleagues and friends Dr. Joao Costa-Nunes from Lisbon University and Dr. Brandon Cline from Strasburg University whose contribution to my experimental life in Lisbon was irreplaceable both on research and personal levels. Special thanks to both of them for contributing to my skills in speaking English (more work to be done by them).

Finally, I am much happy to have a chance to express my appreciation of having constant support from my "brothers-in-arms" with my PhD saga: PhD students Alexsander Trofimov, Ekaterina Veniaminova and Daria Vinogradova. Over the years, they have provided me a reliable support and help with my work, friendly advice and assistance in finding a reason to laugh when I needed this the most. A time that we have shared in Lisbon, Lyon and Wuerzburg are not to forget! I am very grateful to my friends Oleg Vasiliev, Nataliia Sipiagina and Yuriy Manakin who seemingly have believed in me more than I did that, I feel, has eventually brought me to this point of defending my PhD Thesis in the Maastricht University.

Last but not the least, I would like to address my warmest thanks to my beloved parents Andrey and Elena Ivanov, my dear sister Olga Vorob'eva and uncle Kostya (Dr. Konstantin Guriyanov), for supporting me across the last years and encouraging me to achieve my most challenging goals. 


\section{About the author}

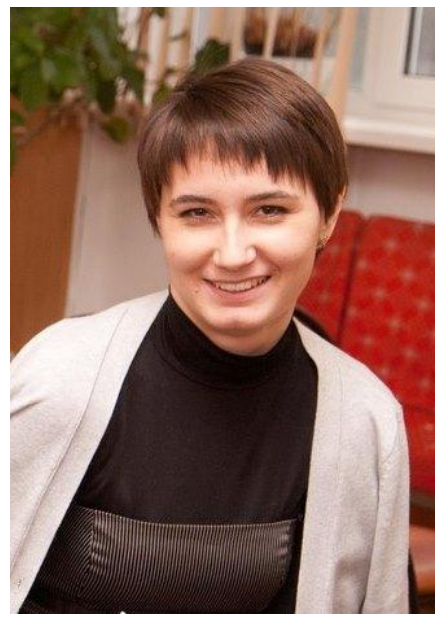

Nataliia Markova was born on April 9th, 1989 in Ivanovo (Russian Federation) and graduated from Ivanovo State University at 2011.

Since 2011, she became actively involved in the collaborative project between the Institute of Physiological Active Compounds of Russian Academy of Sciences (Chernogolovka, Russian Federation) and Department of neuroscience, Maastricht University, which was coordinated by Prof. Steinbusch, Prof. Bachurin, Dr. Elena Schevtsova and Dr. Tatyana Strekalova. During PhD studies, Nataliia has been focusing on the neurobiology of cognition and depression, molecular pathways of glycogen synthase kinase 3 beta (GSK3 $\beta$ ), mechanisms of action of antidepressants and memory enhancers, the role of NMDA- and AMPAreceptors in memory and depression.

Nataliia has acquired numerous techniques in behaviour, brain dissection and surgery, histology and immunohistochemistry, as well as molecular biology through her local and international internships in labouratories of St. Petersbourg, Russian Federation (Institute of Experimental Medicine, Prof. Olga Zubareva), University of Wuerzburg, Germany (Prof. Klaus-Peter Lesch), Lisbon University, Portugal (Dr. Joao Costa-Nunes), C. Bernard University in Lyon, France (Prof. Raymond Cespuglio). She gained her work experience form Prof. Daniel C. Anthony (University of Oxford, UK), Dr. Brandon H. Cline (University of Strasbourg, France) under general supervision of Prof. Harry W.M. Steinbusch and Dr. Tatyana Strekalova from Maastricht University.

Tel: +7 (926) 6734651

E-mail: markova_n.a@list.ru 
Employment:

Since 01.11.2016 - researcher at the Laboratory of Psychiatric Neurobiology, I.M. Sechenov First Moscow State Medical University (0.5 fte).

Since 01.04.2015 - the Department of Neuroscience, School for Mental Health and Neuroscience, Maastricht University (0 fte).

Since 01.02.2014 - researcher at the Laboratory of Cognitive Dysfunctions, Institute of General Pathology and Pathophysiology, Russian Academy of Medical Sciences ( $0.5 \mathrm{fte}$ ).

Since 07.10.2011 - researcher at the Laboratory of Biomolecular Screening, Institute of Physiologically Active Compounds, Russian Academy of Sciences.

Training experiences in other research Institutes:

Since 01.10.2016 - traineeship at the Division of Molecular Psychiatry, University of Wurzburg, Wurzburg, Germany. Study of new pro-neurogenetic drugs in behavioural models and neurogenesis assay - supported by German Academic Exchange Service (DAAD).

November 2016 - traineeship at the Faculty of Medicine, Claude Bernard University,

Lyon, France. Study of neurobiological basis of aggression using TPH2+/mice during stress.

July-September .2015 - traineeship at the Division of Molecular Psychiatry, University of Wurzburg, Wurzburg, Germany. Study of the effects of thiamine and benfotiamine on cognition and GSK3 $\beta$-associated changes in mice.

September 2015 - traineeship at the Faculty of Medicine, Claude Bernard University, Lyon, France. Study of the depressive-like behaviour of SERT -/+ mice exposed to Western diet or ultrasound stress. 
June-December 2014 - traineeships at the Institute for Hygiene and Tropical Medicine New University of Lisbon, Lisbon, Portugal. Study of depressive-like behaviour and contextual memory of SERT-/+ and THP2-/+ mice.

November 2012, May-September 2013, March 2014 - traineeship at the Institute of Experimental Medicine, Russian Academy of Sciences. StPetersburg, Russia. Study of gene expression of GSK3 $\beta$ during forced swimming

Conferences Attended:

[1]. Markova N, Shevtsova E, Vignisse, Zubareva O, Anthony D, Bettendorff L, Lesch KP, Strekalova T. Modified swim test as a model of enhanced contextual conditioning during depression: expression of GSK3 beta and effects of antidepressant treatment. 12th Göttingen Meeting of the German Neuroscience Society. Göttingen. Germany. 22-25.03.2017. Poster presentation.

[2]. Markova N. Modified swim test as a mouse depression paradigm of enhanced cognitive processing: the role of GSK3 $\beta$. Laboratory of Psychiatric Neurobiology, Institute of Molecular Medicine, Mini-conference. I.M. Sechenov First Moscow State Medical University. 16.12.16. Oral presentation.

[3]. Markova N, Shevtsova E, Zubareva O, Cline B, Trofimov A, CostaNunes J, Veniaminova E, Steinbusch H, Bachurin S, Lesch KP, Strekalova T. GSK3b expression in prefrontal cortex correlates with individual differences in floating behaviour in the modified mouse swim test. 22nd Multidisciplinary ISBS International Neuroscience and Biological Psychiatry "Stress and Behaviour" Conference. St-Petersburg, Russia. 16-19.05.2015. Oral presentation.

[4]. Markova N, Shevtsova E, Vignisse J, Zubareva O, Cline B, Trofimov A, Steinbusch H, Bachurin S, Bettendorff L, Lesch KP, Strekalova T. Individual mouse differences in floating predict changes of brain GSK3 $\beta$ : towards further validation of the modified mouse swimming test as a new depression paradigm. 11th Göttingen Meeting of the German Neuroscience Society. Göttingen. Germany. 18-21.03.2015. Poster presentation. 
[5]. Markova N, Shevtsova E, Vignisse J, Zubareva O, Trofimov A, Steinbusch A, Bachurin S, Bettendorff L, Strekalova T. Thiamine and benfotiamine improve memory, decrease depressive-like behaviour and reduce brain expression of GSK3 $\beta$ in mice. The 8th International Conference on Thiamine: From Catalysis to Pathology. Liege, Belgium. 23-26.05.2014. Poster and oral presentations.

[6]. Markova N, Shevtsova E, Bachurin S, Steinbusch HMW, Strekalova T. Individual susceptibility to depressive-like traits in two mouse models of depression and hippocampal levels of GSK3 $\beta$. The 38th FEBS Congress. Saint Petersburg, Russia. 06-11.06.2013. Poster presentation.

[7]. Markova N, Bolkunov A, Proshin A, Grigoriev V, Bachurin S, Strekalova T. Effects of memantine and new potentiator of AMPA receptors QXX on contextual memory and its extinction in mice C57BL/6N. II AllRussian Conference with International Participation "Hippocampus and memory: norm and pathology". Pushchino, Russia. 10-14.09.2012. Poster presentation.

[8]. Markova N, Strekalova T, Nikolaeva N, Vankin G, Bolkunov A, Bachurin S. Search for cognitive-stimulating agents among fluorinecontaining gamma-carbolines. VIII International interdisciplinary congress "Neuroscience for medicine and psychology". Ukraine, Sudak. 0212.06.2012. Poster presentation.

[9]. Markova N, Grigoriev S, Bolkunov A, Bachurin S, Strekalova T. The effect of new derivatives of thiourea, acting simultaneously on the AMPA and NMDA receptors, on memory mice in the two tests with sensitivity to different subtypes of glutamate receptor. XII Congress "Young science in a classical university". Ivanovo, Russia. 23-24.04.2012. Oral presentation.

Awards:

Prizes for the best oral presentations at the 1st, 2nd and 3nd Conferences of young scientists in the Institute of Physiologically Active Compounds RAS. 2014-2016. Chernogolovka, Russia. (200 EUR) 
Prize for the best poster at the 8th International Conference on Thiamine: From Catalysis to Pathology. Liege, Belgium, 23-26.05.2014. (100 EUR).

Travel Award for Youth Travel Fund grants to attend the 38th FEBS Congress. Saint Petersburg, Russia, 06-11.06.2013 (250 EUR).

First prize for the best report at XII Congress "Young science in a classical university". Ivanovo, Russia, 23-24.04.2012.

\section{Funding Secured}

Scholarship from German Academic Exchange Service (DAAD) for 10 months (01.10.2016 - 31.07.2017, 13000 EUR in total)

Grant of Russian Foundation of Basic Research "The role of plasticity and GSK-3b cascades in the mechanisms of depression: a study on mutant mice SERT and TPH2 with genetic deficit of neuronal serotonin" for 2 years (01.01.2016-31.12.2017, 7200 EUR / year) 


\section{List of publications}

[1]. Veniaminova, E., Cespuglio, R., Cheung, C., W., Umriukhin, A., Markova, N., Shevtsova, E., Lesch, K., P., Anthony, A., Strekalova, T., 2016. Autism-like behaviours and memory deficits accompany a Western diet in mice. Neural Plasticity (accepted for publication).

[2]. Couch, Y., Trofimov, A., Markova, N., Nikolenko, V., Steinbusch, H.W., Chekhonin, V., Schroeter, C., Lesch, K.-P., Anthony, D.C., Strekalova, T., 2016. Low-dose lipopolysaccharide (LPS) inhibits aggressive and augments depressive behaviours in a chronic mild stress model in mice. J. Neuroinflammation 13, 108-125.

[3]. Markova, N., Bazhenova, N., Anthony, D.C., Vignisse, J., Svistunov, A., Lesch, K.-P., Bettendorff, L., Strekalova, T., 2016. Thiamine and benfotiamine improve cognition and ameliorate GSK3 $\beta$-associated stress-induced behaviours in mice. Prog. Neuro-Psychopharmacology Biol. Psychiatry. 75, 148-156.

[4]. Markova, N., Chernopiatko, A., Kubatiev, A., Bachurin, S., Steinbusch, H. M.W., Strekalova, T., 2016. A study of the effects of 3,5-diiodo-Lthyronine in the tail suspension and forced swim models of depression. Transl. Neurosci. Clin. 2, 96-107.

[5]. Markova, N., Strekalova, T., Shevtsova, E., Zubareva, O., Bakhmet, A., Steinbusch, H.M., Bachurin, S., Lesch, K.-P., 2016. Individual Differences in Behavioural Despair Predict Brain GSK3 $\beta$ Expression in Mice: The Power of a Modified Swim Test. Neural Plast. 2016, 5098591.

[6]. Cline, B.H., Costa-Nunes, J.P., Cespuglio, R., Markova, N., Santos, A.I., Bukhman, Y. V, Kubatiev, A., Steinbusch, H.W.M., Lesch, K.-P., Strekalova, T., 2015. Dicholine succinate, the neuronal insulin sensitizer, normalizes behaviour, REM sleep, hippocampal pGSK3 $\beta$ and mRNAs of NMDA receptor subunits in mouse models of depression. Front. Behav. Neurosci. 9-37.

[7]. Costa-Nunes, J.P., Cline, B.H., Araújo-Correia, M., Valença, A., Markova, N., Dolgov, O., Kubatiev, A., Yeritsyan, N., Steinbusch, H.W.M., Strekalova, T., 2015. Animal Models of Depression and Drug Delivery with Food as an Effective Dosing Method: Evidences from Studies with Celecoxib and Dicholine Succinate. Biomed Res. Int. 2015, 596126. 
[8]. Markova, N., Chernopiatko, A., Schroeter, C.A., Malin, D., Kubatiev, A., Bachurin, S., Costa-Nunes, J., Steinbusch, H.M.W., Strekalova, T., 2013. Hippocampal gene expression of deiodinases 2 and 3 and effects of 3,5diiodo-L-thyronine T2 in mouse depression paradigms. Biomed Res. Int. 2013, 565218.

[9]. Markova, N., Shevtsova, E., 2013. Stress as cause of depression and Alzheimer's disease: the role of glycogen synthase kinase-3 beta. Pathogenesis. 9, 4-10.

[10]. Redkozubova, O., Bolkunov, A., Van 'kin, G., Revishchin, A., Eritsan, N., Markova, N., Bachurin, S., Strekalova, T., 2013. Occurrence of hedonic and lack of affective deficit in 18-month-old C57BL/6 mice implications for modeling elderly depression. Pathogenesis. 11, 64-9.

\section{Papers under revision}

[1]. Strekalova, T., Bazhenova, N., Trofimov, A., Schmitt. A., Markova, N., Grigoriev, V., Zamoyskii, V., Serkova, T., Vinogradova, D., Lilesaar, C., Umriukhin A., Schevtsova E., Lesch K.P., Bachurin S. Pro-neurogenic, memory-enhancing and anti-stress effects of a novel fluorine analogue dimebon DF-302: possible roles of 5-HT6- and AMPA receptor-mediated mechanisms. Mol. Neurobiol.

[2]. Vignisse, J., Sambon, M., Caron, N., Malgrange, B., Shevtsova, E., Svistunov, A., Anthony, D., C., Markova, N., Bazhenova, N., Coumans, B., Lakaye, B., Wins, P., Strekalova, T., Bettendorff, L. Thiamine and benfotiamine prevent stress-induced suppression of hippocampal neurogenesis in mice exposed to predation without affecting brain thiamine diphosphate levels. Mol. Cell. Neuroscience.

\section{Papers in preparation}

[1]. Markova, N., Rutten, B., P., F., Shevtsova, E., Ludinova, V., Svistunov, A., Lesch, K., P., Strekalova, T. Unrevealing the functions of brain GSK3 $\alpha$ : possible synergy with GSK3 $\beta$-upregulation and reversal by an antidepressant treatment in a mouse depression model. Journal of Affective Disorders. 